\title{
The Integration of Heat Pumps and Thermal Storage for Residential Demand Side Management
}

\author{
by \\ Christopher Baldwin \\ B.Eng., Carleton University, 2011 \\ M.A.Sc., Carleton University, 2014
}

A thesis submitted to the Faculty of Graduate and Postdoctoral Affairs in partial fulfillment of the requirements for the degree of

\author{
Ph.D. \\ in
}

Mechanical Engineering

Carleton University

Ottawa, Ontario

(C) 2020, Christopher Baldwin 


\section{Abstract}

Space heating and cooling accounts for approximately $65 \%$ of residential secondary energy consumption, of which almost $40 \%$ is met using electricity in Canada. This demand places a significant peak load on the electrical grid that must be managed to reduce the required generating and transmission capacity within the grid. It is proposed that this demand side management can be achieved using thermal storage coupled with a heat pump to charge during off-peak periods and use the stored heating and cooling during peak periods. Through this work, TRNSYS Types were validated to model the performance of a liquid to liquid heat pump, medium temperature chiller and using sensible storage tanks and stratified cold thermal storage. A new TRNSYS Type was developed and validated to model a compact ice storage system to store cooling potential for peak periods. These components were combined, and the coupled performance of the heat pump connected to both hot and cold thermal storage was determined. It was found that higher flow rates resulted in better performance, when compared to using low flow rates that resulted in the stratification in the storage tanks. The complete system was then integrated into a house modelled situated in Ottawa and different combinations were examined for the total energy consumption, peak consumption, annual costs, and greenhouse gas emissions. In all cases, total consumption, energy costs and greenhouse gas emissions increased, although certain combinations showed greater potential, with the greatest potential being the offsetting of peak cooling loads, when compared to heating loads. This system was then implemented in locations across North America, with different rate structures and climactic conditions. It was found that locations with a high difference between the peak and off-peak rate, and high cooling loads had the greatest potential for reducing peak consumption and reducing 
utility costs. When looking at life cycle costs, rate increases selected over the 15-25-year period examined greatly influenced the economics. In conclusion, the system is technically feasible, where a large portion of cooling peak loads can be offset, but to be economically feasible, government incentives or a high annual rate of utility cost increases must occur. 


\section{Acknowledgements}

I would first like to acknowledge the support of my colleagues both past and present in the Solar Energy System Laboratory, as well as colleagues across the Building Performance Research Centre at Carleton University. There are too many of you to name individually, it has been your support and guidance over the past years has been invaluable as I have completed this work

I would like to thank my supervisor, Dr. Cynthia Cruickshank for the support, patience and guidance she has provided over the course of this research project.

I would like to acknowledge the financial support of the Natural Sciences and Engineering Research Council (NSERC), both through my supervisors Discovery Grant and the Doctoral Scholarship program, as well as the Province of Ontario, through the Ontario Graduate Scholarship.

Finally, I would like to acknowledge the support and encouragement of my friends and family throughout my academic career. 


\section{Table of Contents}

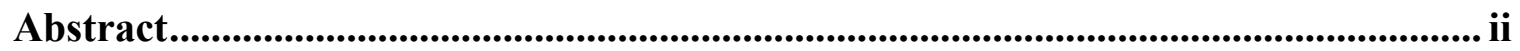

Acknowledgements ........................................................................................................................... iv

Table of Contents ................................................................................................................................. v

List of Tables .............................................................................................................................. $\mathrm{x}$

List of Figures........................................................................................................................... xiv

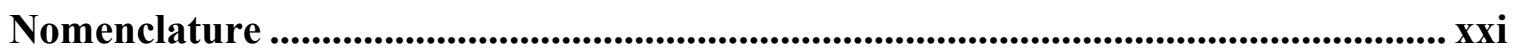

Chapter 1: Introduction ................................................................................................................ 1

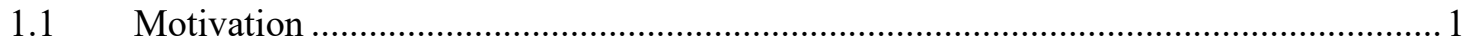

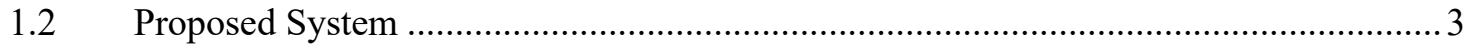

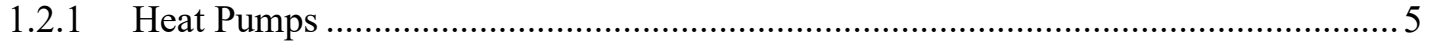

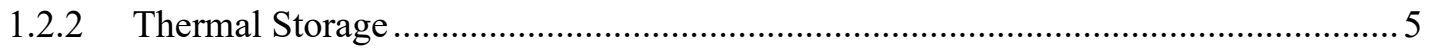

$1.3 \quad$ Research Objectives ........................................................................................

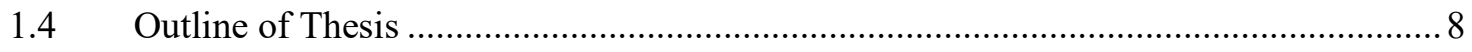

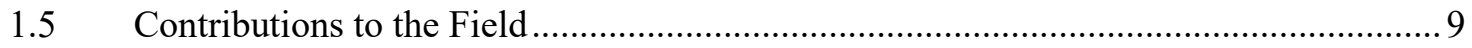

Chapter 2: Background and Literature Review ................................................................ 11

2.1 Shifting Electrical Consumption from Peak to Off-Peak Periods .................................11

2.2 Utility Based Demand Side Management ............................................................... 12

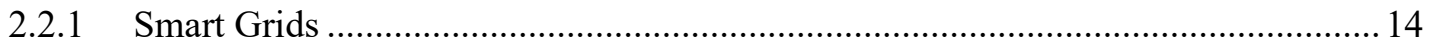

2.3 Consumer Based Demand Side Management.......................................................... 16

2.3.1 Passive Building Design ..................................................................................... 16

2.3.2 Electric Heating with Thermal Storage ............................................................... 18

2.3.3 Heat Pumps with Thermal Storage ........................................................................2 20

$2.4 \quad$ Heat Pumps 


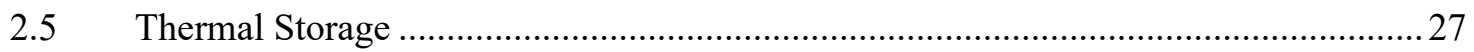

2.5.1 Sensible Thermal Storage - Stratified Storage Tanks .........................................28

2.5.1.1 Modelling Stratified Storage Tanks.......................................................... 30

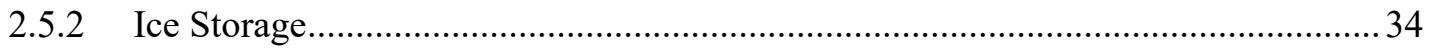

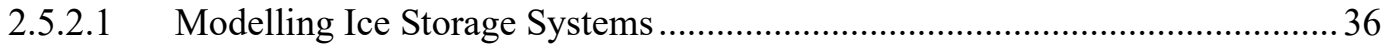

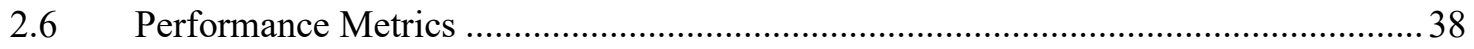

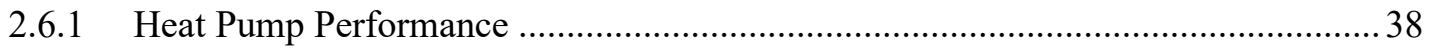

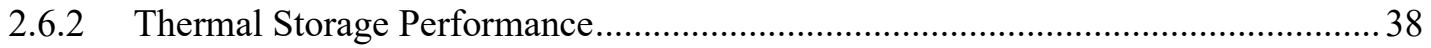

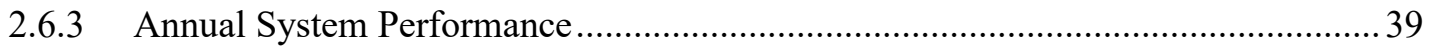

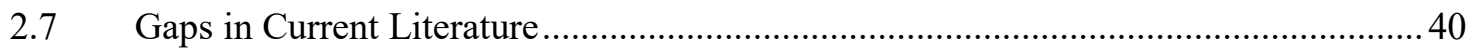

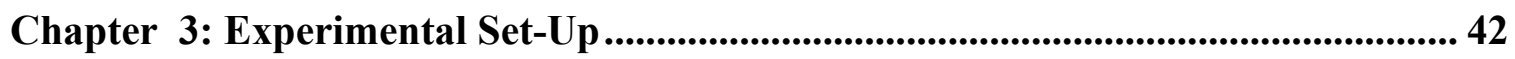

3.1 Liquid to Liquid Heat Pump Coupled with Sensible Thermal Storage ........................ 43

3.2 Medium Temperature Chiller Evaluation........................................................... 49

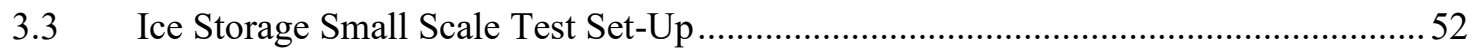

3.3.1 Instrumentation within the Ice Storage Test Set-Up ...........................................54

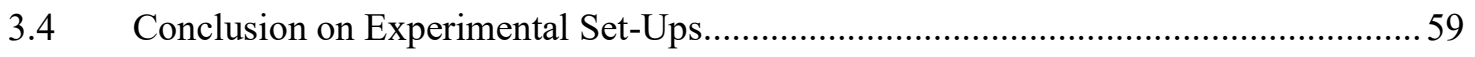

Chapter 4: Component Modelling and Validation ....................................................... 61

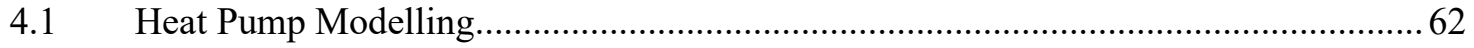

4.1.1 Modelling the Standard Liquid to Liquid Heat Pump..........................................62

4.1.2 Modelling the Medium Temperature Chiller ........................................................ 70

4.2 Modelling Sensible Cold Thermal Storage Tanks................................................... 73

4.2.1 Comparison of TRNSYS Model Types …....................................................... 76

4.2.2 Validation of the Cold Thermal Storage with Water as a Storage Medium............. 78

4.2.3 Validation of the Cold Thermal Storage with a Water/Glycol Solution as a Storage

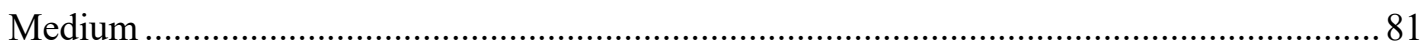

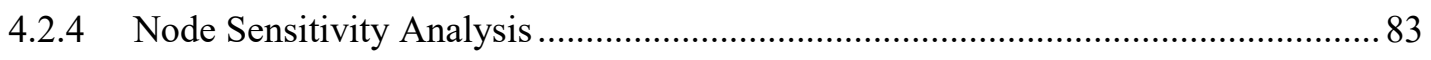


4.3 Modelling Latent Cold Thermal Storage Models - Ice Storage .................................. 86

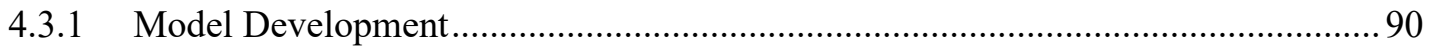

4.3.1.1 Forced Convection in the Pipe …............................................................ 93

4.3.1.2 Conduction through the Pipe Material........................................................... 95

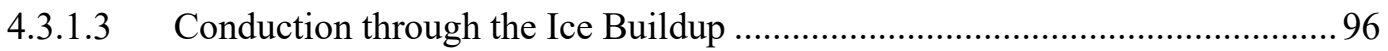

4.3.1.4 Natural Convection between the Pipe/Ice Surface and Tank Fluid................. 96

4.3.1.5 Calculating the Total Heat Transfer .............................................................. 98

4.3.1.6 Modelling the Pipe/Ice Surface Temperature .................................................. 99

4.3.1.7 Modelling Tank Temperature and Ice Formation...................................... 100

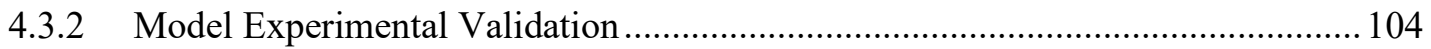

Chapter 5: Whole System and Building Modelling Methodology ............................ 113

5.1.1 Charge Efficiency of the Sensible Thermal Storage ............................................. 113

5.1.1.1 Impact of Flow on Cold Thermal Storage Charging .................................. 114

5.1.1.2 Impact of Flow on Hot Thermal Storage Charging ................................... 117

5.2 Ice Storage System Configuration and Charging Efficiency .................................... 121

5.3 Comparison between Sensible and Latent Cold Storage Efficiency ......................... 125

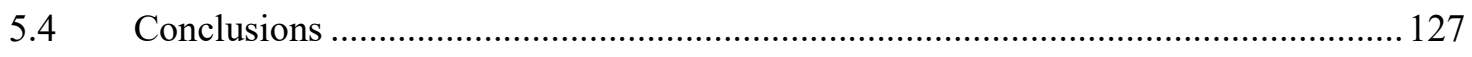

Chapter 6: Annual Modelling Study .......................................................................... 129

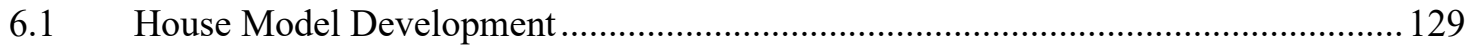

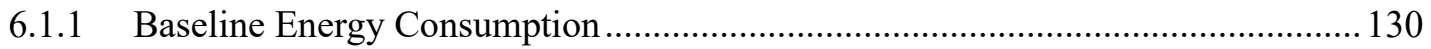

6.1.2 Baseline Energy Consumption Using a Heat Pump ............................................. 132

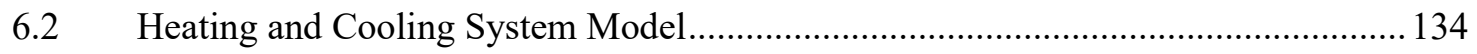

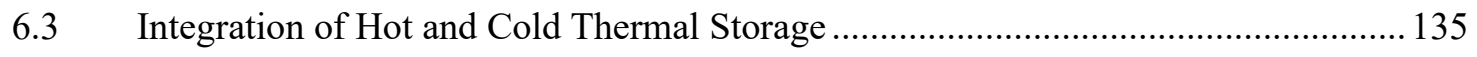

6.4 Basic Set-Point Control of the Thermal Storage Systems ....................................... 138

6.5 Medium Temperature Chiller for Meeting Heating and Cooling Loads .................... 144

6.5.1 Baseline Results Using the Medium Temperature Chiller................................... 144 
6.5.2 Integration of Sensible Cold Thermal Storage .................................................... 145

6.5.3 Integration of an Ice Storage Thermal Storage System....................................... 149

6.6 Integration of Domestic Hot Water Loads ....................................................... 152

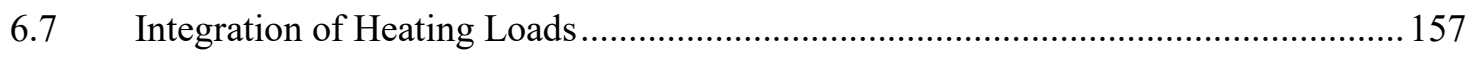

6.8 Utilization of Predictive Controls for Determining Storage Set-Points ..................... 160

6.8.1 Determining Relationship Between Weather Conditions and Loads ..................... 161

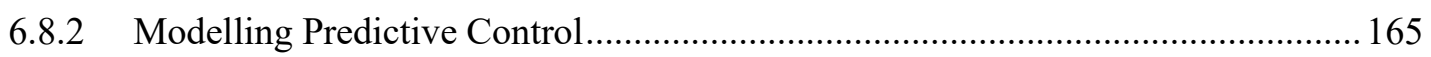

6.8.3 Results using the Predictive Control Strategy for Storing Cooling ....................... 168

6.9 Conclusions on the Potential for Off-Setting Peak Loads in Ottawa ........................ 170

Chapter 7: Analysis of Different Assumptions, Locations and Rate Structures.... 176

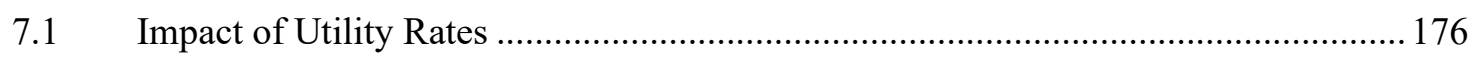

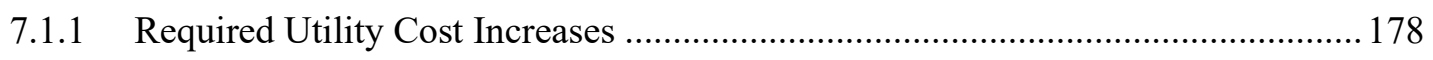

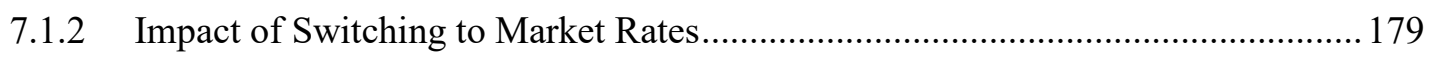

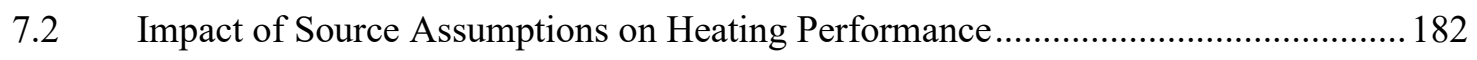

7.3 Potential for Off-Setting Peak Loads across North America.................................... 189

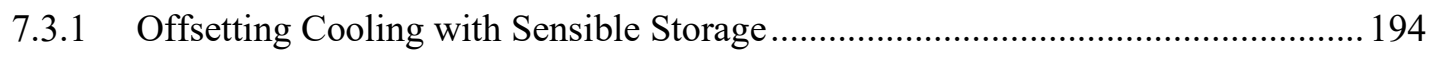

7.3.2 Use of Ice Storage in Multiple Locations Across North America .......................... 199

7.4 Decoupling Heat and Cooling Systems .......................................................... 206

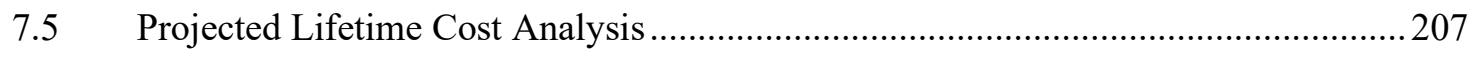

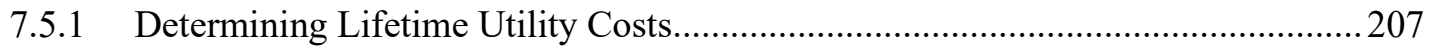

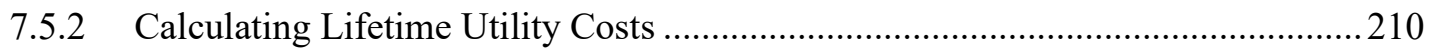

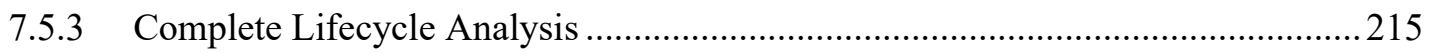

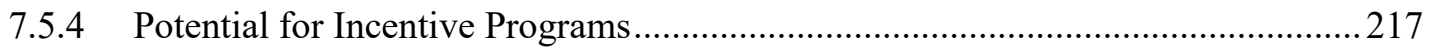

7.6 Conclusions on Potential for the Integrated System............................................219

Chapter 8: Conclusions and Future Work .................................................................... 222

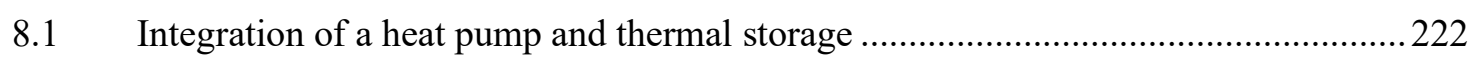


8.2 Annual results and potential for demand side management

8.3 Future Work 230

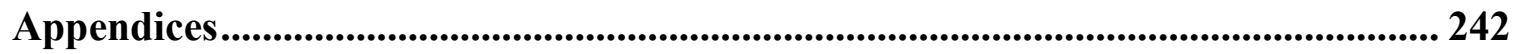

Appendix A Energy Landscape in Canada and Ontario ..................................................... 242

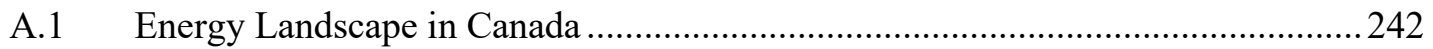

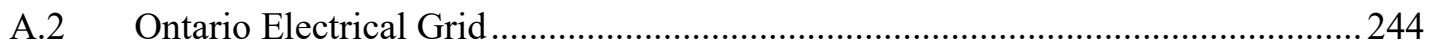

A.3 Influence of Peak Electrical Loads on Greenhouse Gas Emissions ........................246

A.4 Calculating Greenhouse Gas Emissions from Electrical Generation ......................246

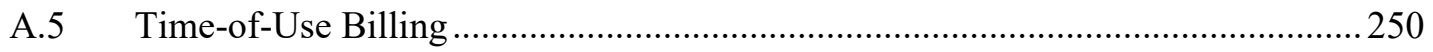

A.6 Relationship between Peak Electrical Consumption and GHG Emissions ............251

Appendix B Heat Pump and Chiller Performance Map ...................................................2253

B.1 Source Side Heat Transfer Fluid - Water ........................................................... 253

B.2 Source Side Heat Transfer Fluid - Water/Glycol Solution ...................................257

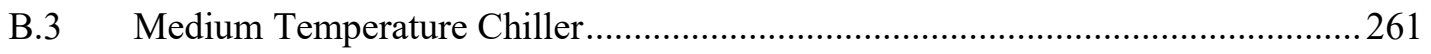

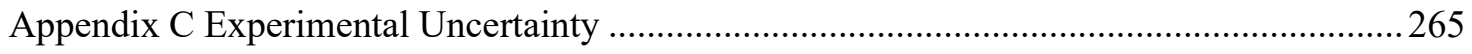

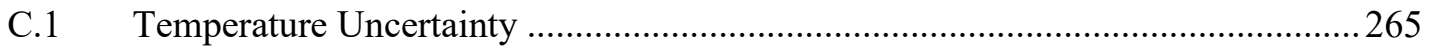

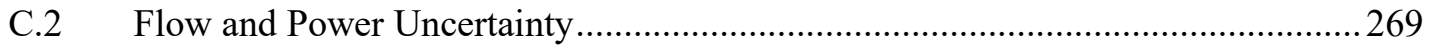

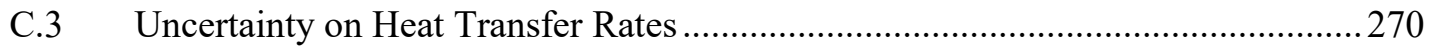

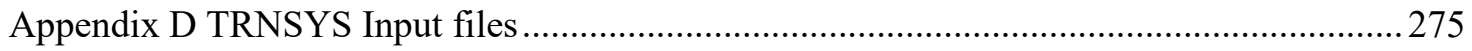

D.1 Heat Pump with a 50/50 water/glycol solution on the source side .......................275

D.2 Medium Temperature Chiller Input Files.........................................................29

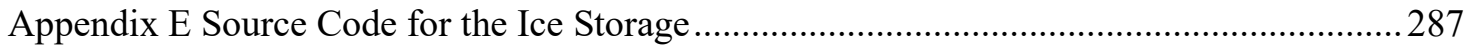

Appendix F Schematics of TRNSYS Models used to model the full building ........................300 


\section{List of Tables}

Table 4-1: Flow rates for each of the experimental test runs.......................................... 64

Table 4-2: Fluid properties of the two storage mediums ................................................. 75

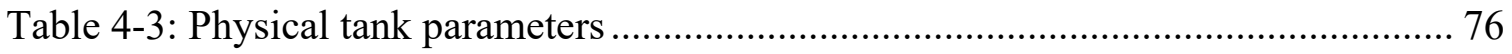

Table 4-4: Constant values for calculating natural convection around a cylinder............ 97

Table 4-5: Experimental parameters of the small-scale ice storage set-up..................... 105

Table 4-6: Results for the absolute differences for each Nusselt number modelled ...... 106

Table 5-1: Results at the end of each test with varying flow rates - Heat Pump ........... 116

Table 5-2: Results at the end of each test with varying flow rates - Chiller .................. 116

Table 5-3: Results at the end of each modelled hot flow rates with the heat pump ....... 120

Table 5-4: Results at the end of each modelled hot flow rates with the chiller.............. 121

Table 5-5: Summary of results for different pipe configurations ................................... 123

Table 5-6: Impact of different storage charge levels using the ice storage system ........ 124

Table 6-1: House model parameters ……………………............................................ 130

Table 6-2: Baseline energy consumption by time, heating costs and greenhouse gas

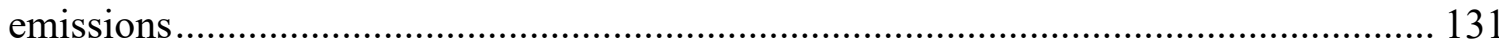

Table 6-3: Baseline energy consumption using a heat pump for space heating and cooling

Table 6-4: Results of the optimal configuration looking at just heating and cooling..... 142

Table 6-5: Base building loads using the medium temperature chiller ......................... 144

Table 6-6: Storage densities for different volumes and set-points relative to $17^{\circ} \mathrm{C}$....... 146

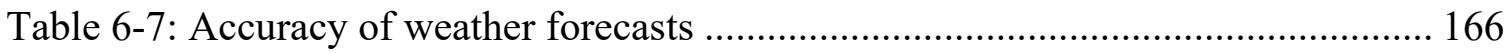

Table 6-8: Summary of results for off-setting peak loads in Ottawa............................. 173 
Table 7-1: Comparison of utility rates over the course of the study......

Table 7-2: Energy costs using current utility rates

Table 7-3: Annual costs based on changing utility rates ........................................ 178

Table 7-4: Average market electrical rates by month and rate period in $\$ / \mathrm{MWh}$......... 179

Table 7-5: Annual Utility costs using the Ontario market rate ................................... 180

Table 7-6: Annual costs to meet the space cooling and DHW load ............................. 181

Table 7-7: Comparison between base case and system using ice storage and the medium

temperature chiller at different source temperatures .............................................. 187

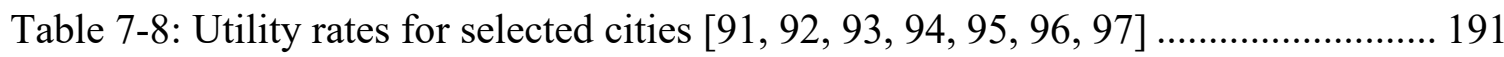

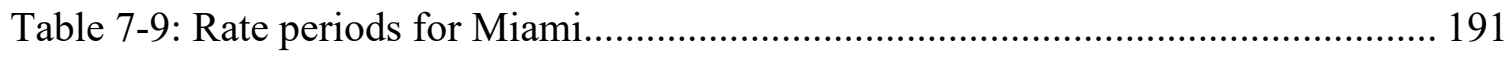

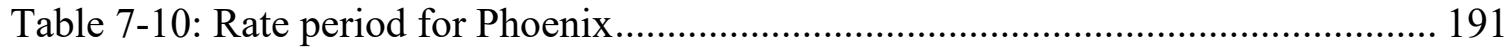

Table 7-11: Rate periods for Los Angeles .............................................................. 191

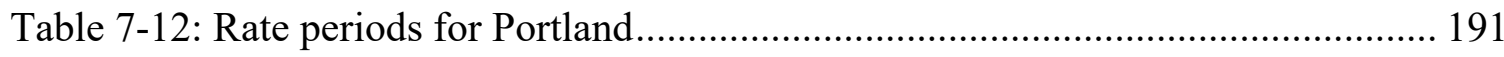

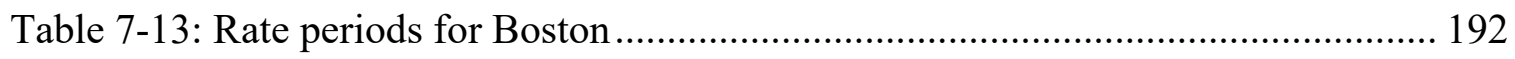

Table 7-14: Rate periods for Toronto and Sudbury ................................................ 192

Table 7-15: Optimal parameters and the comparison between base costs and using

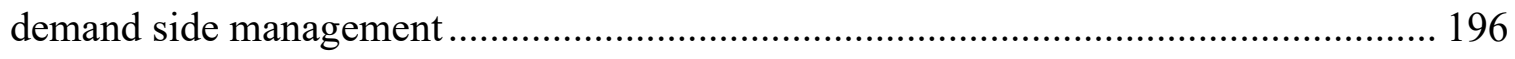

Table 7-16: Equations for each of the cities relating max daily temperature in degrees

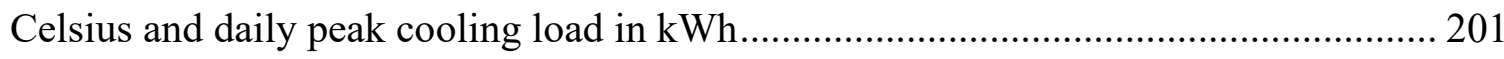

Table 7-17: Comparison of energy costs using both sensible and latent storage .......... 201

Table 7-18: Optimal sensible storage capacity by location .................................... 203

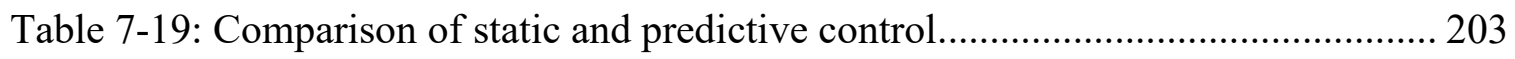

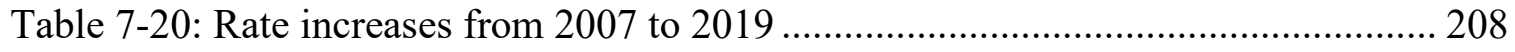


Table 7-21: Potential rate increases over the next 25 years 209

Table 7-22: Projected utility rates over the next 25 years with different increase scenarios 210

Table 7-23: Annual and total utility costs for the base system over the next 25 years (all values in \$) 211

Table 7-24: Comparison between total utility costs to meet space cooling and DHW loads over the next 25 years - All values in Canadian Dollars 215

Table 7-25: Lifecycle cost analysis using two different rate structures - All values in Canadian Dollars 217

Table A-1: Greenhouse gas intensity by generating source in $\mathrm{g} \cdot \mathrm{CO}_{2}$ equivalent $/ \mathrm{kWh} . .248$

Table A-2: Classification of usage periods in Ontario 251

Table A-3: Greenhouse gas intensity by season and time-of-use .............................. 252

Table B-1: Electrical performance map for the heat pump $(\mathrm{kW})$............................... 254

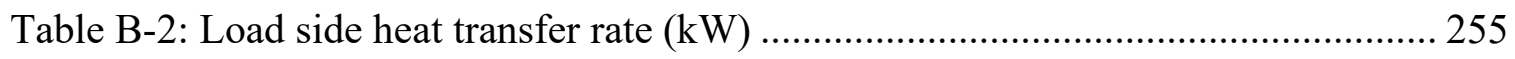

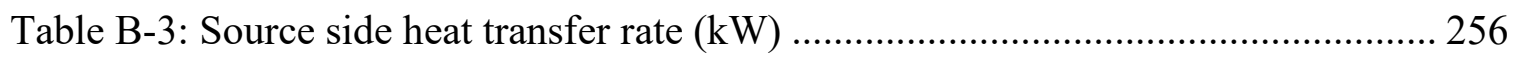

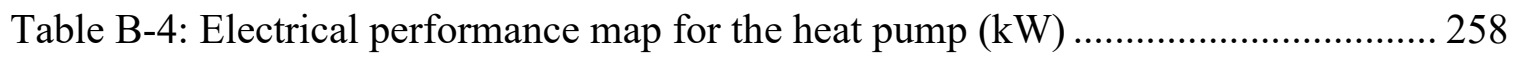

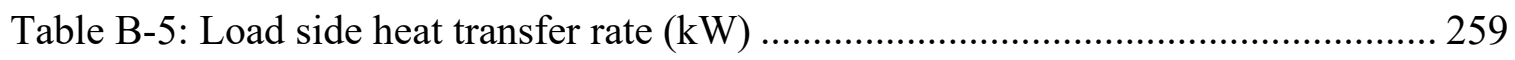

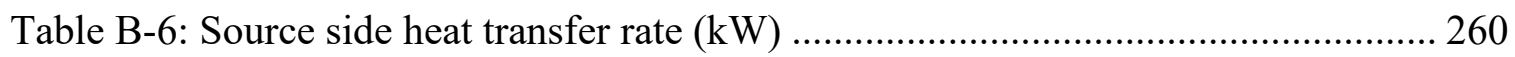

Table B-7: Electrical performance map for medium temperature chiller $(\mathrm{kW})$............ 262

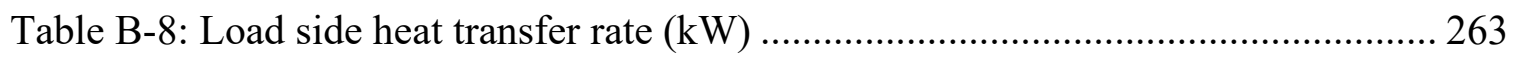

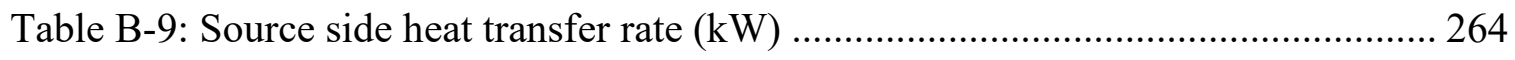

Table C-1: Experimental uncertainty on the load side heat transfer rate $(\mathrm{kW})$ with water

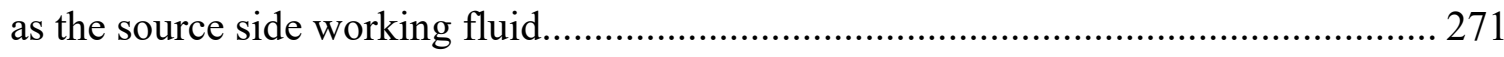


Table C-2: Experimental uncertainty on the source side heat transfer rate $(\mathrm{kW})$ with water

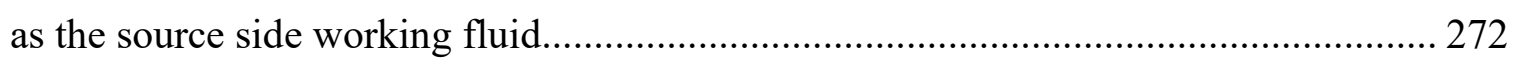

Table C-3: Experimental uncertainty on the load side heat transfer rate $(\mathrm{kW})$ with a 50/50 water/glycol solution by volume as the source side working fluid............................. 273 Table C-4: Experimental uncertainty on the source side heat transfer rate $(\mathrm{kW})$ with a 50/50 water/glycol solution by volume as the source side working fluid.

Table D-1: Text files for modelling the heat pump using a glycol/water solution on the cold side 275 Table D-2: Input files for the medium temperature chiller 279 


\section{List of Figures}

Figure 1-1: High level schematic of the proposed system with the heat pump connected to a hot and cold thermal storage system......

Figure 2-1: Schematic of energy into and out of a single node being modelled in a stratified thermal storage tank 32

Figure 2-2: Energy storage densities for cold thermal storages.

Figure 3-1: Photo of heat pump (center) with cold (left) and hot (right) thermal storage systems

Figure 3-2: Schematic of completed experimental test set-up

Figure 3-3: Thermocouple probe as it enters the tank (Left) and the thermocouple installed within the tank (Right) 48

Figure 3-4: Example of a temperature profile at low flow rates in the cold thermal storage

Figure 3-5: Images of the medium temperature chiller set-up, including the components of the chiller (top) and the required infrastructure to operate and test the chiller (bottom)

Figure 3-6: Thermocouple epoxied to the outer surface of the submerged pipe 56

Figure 3-7: Thermocouples connected to fishing line to allow the fluid temperature around the pipe to be measured. 57

Figure 3-8: Images of the completed small-scale ice storage set-up

Figure 4-1:Comparison of experimental and simulated performance of the heat pump with water (left) and water/glycol solution (right) with flow rates as indicated in Table 41. 
Figure 4-2: Comparison of instantaneous heat transfer rates and electrical consumption Water 67

Figure 4-3: Comparison of cumulative heat transferred and electrical consumption -

Water 67

Figure 4-4: Comparison of instantaneous heat transfer rates and electrical consumption -

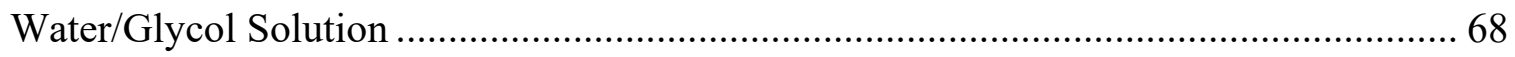

Figure 4-5: Comparison of cumulative heat transferred and electrical consumption $-\ldots .68$

Figure 4-6: Comparison of outlet temperatures for the medium temperature chiller at A) 9

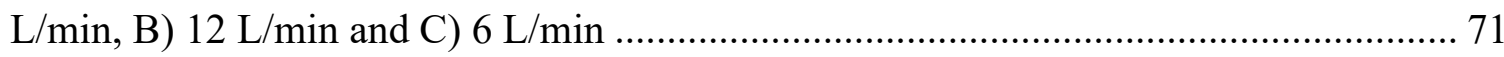

Figure 4-7: Instantaneous heat transfer and electrical consumption for the chiller ......... 72

Figure 4-8: Cumulative heat transfer and electrical consumption for the chiller ............ 72

Figure 4-9: Comparison between TRSNYS Types with water as the storage medium.... 77

Figure 4-10: Comparison between TRSNYS Types with a water/glycol solution as the

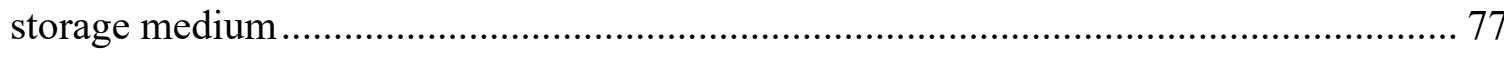

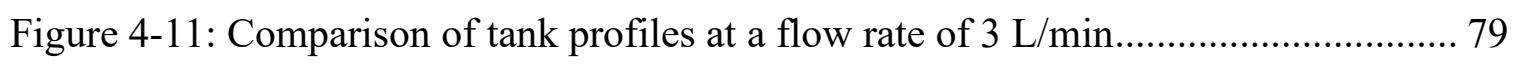

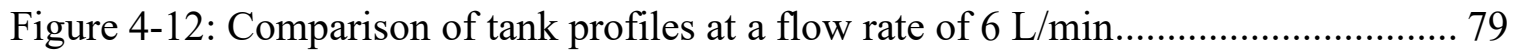

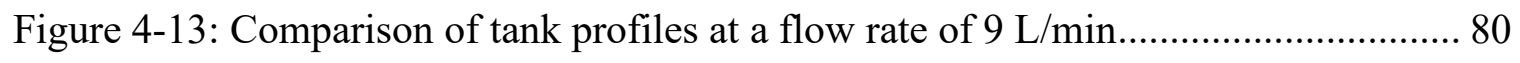

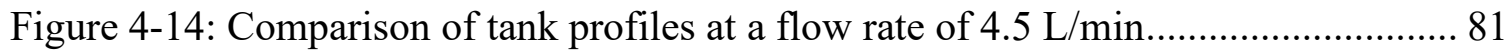

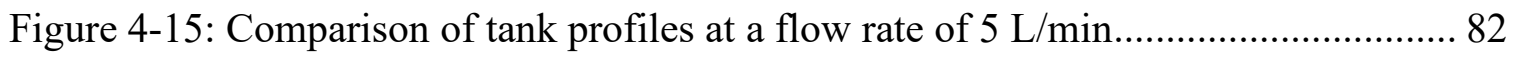

Figure 4-16: Comparison of tank profiles at a flow rate of $7.5 \mathrm{~L} / \mathrm{min}$.......................... 82

Figure 4-17: Comparison of number of nodes with water as the storage medium ........... 84

Figure 4-18: Comparison of number of nodes with a glycol solution as the storage

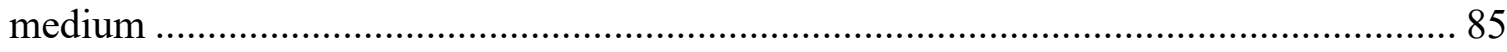


Figure 4-19: Pipe configuration within the ice storage system................................... 91

Figure 4-20: Node configuration on a single submerged pipe .................................. 92

Figure 4-21: Schematic of the submerged pipe, with ice build up, and heat transfer

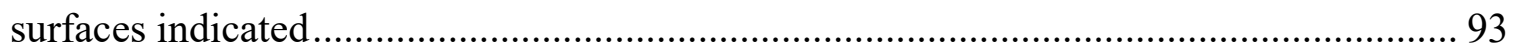

Figure 4-22: Comparison of different Nusselt numbers to the experimental values ...... 106

Figure 4-23: Comparison of pipe outlet temperature for two experimental runs .......... 108

Figure 4-24: Comparison of heat transfer rates for two experimental runs .................. 108

Figure 4-25: Comparison of total energy transfer for two experimental runs ............... 108

Figure 4-26: Comparison between modelled and experimental average tank temperature

Figure 4-27: Comparison between modelled and experimental ice thickness............... 110

Figure 4-28:Comparison between modelled and experimental stored cooling potential 111

Figure 5-1: Comparison of cold flows for the standard heat pump ........................... 115

Figure 5-2: Comparison of cold flows for the medium temperature chiller .................. 115

Figure 5-3: Comparison of hot flows for the heat pump ......................................... 119

Figure 5-4: Comparison of hot flows for the medium temperature chiller.................... 119

Figure 5-5: Comparison of cooling potential stored and COP between sensible and latent

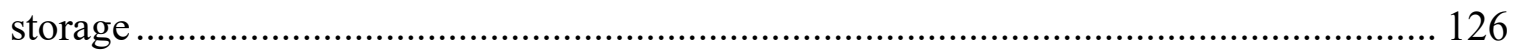

Figure 5-6: Comparison of inlet temperature and heat transfer rates between sensible and

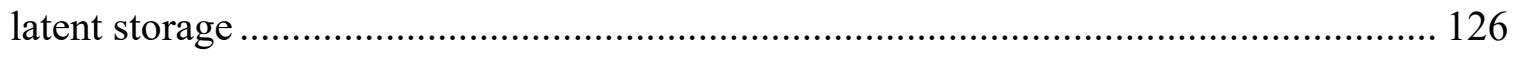

Figure 6-1: Control schematic for the proposed system as modelled ......................... 135

Figure 6-2: Simulation results for varying source and load flow rates ........................ 136

Figure 6-3: Simulation results for varying hot and cold tank set-points...................... 137 
Figure 6-4: Simulation results varying off-peak tank set-points 139

Figure 6-5: Impact of changing thermal storage volumes on total energy consumption 140 Figure 6-6: Impact of changing thermal storage volumes on peak electrical consumption

Figure 6-7: Impact of changing thermal storage volumes on annual utility costs

Figure 6-8: Annual electrical consumption for different cold storage volumes and setpoints

Figure 6-9: Annual utility costs and greenhouse gas production over different storage volumes and set-points

Figure 6-10: Electrical consumption to meet the space cooling demand only 148

Figure 6-11: Electrical consumption using an ice storage system with different set-points

Figure 6-12: Electrical consumption to meet cooling demand using an ice storage system with different set-points 150

Figure 6-13: Annual cost and greenhouse gas production with an ice storage system and different set-points 151

Figure 6-14: Annual electricity consumption to meet space heating, cooling, and DHW loads - Integrated DHW 153

Figure 6-15: Annual utility costs and greenhouse gas emissions - Integrated DHW ... 154 Figure 6-16: Electrical consumption to the meet the space cooling and DHW demands of the building 155

Figure 6-17: Impact of pre-heat tank volume on annual consumption for space cooling and DHW 156 
Figure 6-18: Impact of pre-heat tank volume on annual utility costs 156

Figure 6-19: Annual energy consumption when a sensible hot storage system is integrated

Figure 6-20: Annual utility costs and greenhouse gas emissions using sensible hot storage

Figure 6-21: Plot of daily maximum temperature and cooling load(left), and heating load (right) 162

Figure 6-22: Cooling load compared to daily high temperature, with steps added to trendline 163

Figure 6-23: Relationship between heating load and daily minimum temperature ....... 164

Figure 6-24: Comparison of actual and forecasted daily highs for the month of July.... 167

Figure 6-25: Annual consumption using a predictive control system 168

Figure 6-26: Electrical costs and greenhouse gas production using a predictive control system 169

Figure 7-1: Electrical consumption by time of use for different source temperatures ... 184 Figure 7-2: Electrical consumption by end use for different source temperatures 185 Figure 7-3: Electrical consumption by time of use for the medium temperature chiller with different source temperatures. 186

Figure 7-4: Selected cities and the ASHRAE climate map for North America. 190

Figure 7-5: Annual electrical consumption by end use 193

Figure 7-6: Annual consumption and cost by time of use 193

Figure 7-7: Impact of changing set-point and tank volume and annual utility rates for Phoenix 195 
Figure 7-8: Impact of changing set-point and tank volume and annual utility rates for Portland 195

Figure 7-9: Comparison of electrical consumption by time of use 197

Figure 7-10: Comparison of annual electrical cost by time of use 198

Figure 7-11: Relationship between peak cooling load and maximum daily temperature for Portland 200

Figure 7-12: Comparison of energy consumption between latent and sensible storage based on time of use 202

Figure 7-13: Comparison of Charge performance to store the same quantity of cooling potential. 205

Figure 7-14: Comparison of annual utility costs per year for the next 25 years 211

Figure 7-15: Comparison of total utility costs over the next 25 years. 212

Figure 7-16: Annual utility costs to meet cooling and DHW loads for the next 25 years 213

Figure 7-17: Total cost to meet space cooling and DHW loads over the next 25 years. 214 Figure A-1: Electrical generating source by Canadian province 242

Figure A-2: Total Canadian electrical generation by month 243

Figure A-3: Hourly electrical generation by source in Ontario for 2015 245

Figure A-4: Average historical electrical consumption by hour in Ontario (2002-2015)

Figure A-5: Hourly greenhouse gas emissions in Ontario on an hourly basis and by generating source for 2015 249

Figure A-6: Greenhouse gas intensity by hour of the day - Ontario 2015. 250 


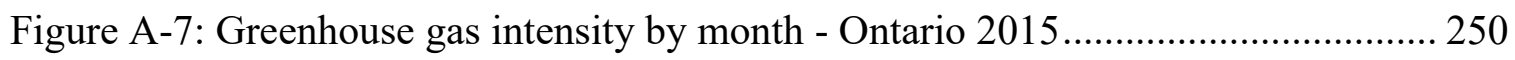

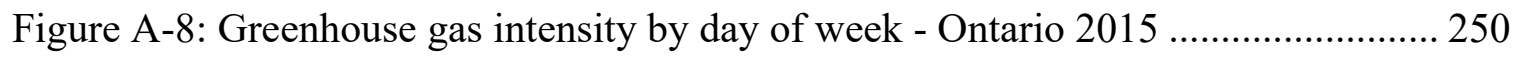

Figure A-9: Greenhouse gas intensity compared to total consumption and time-of-use 252

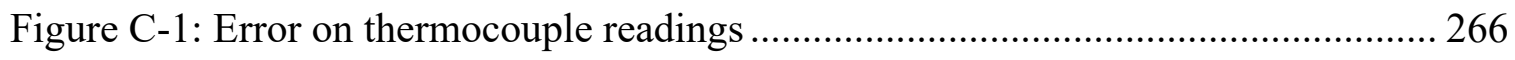

Figure C-2: Thermocouple error after removing equipment bias .............................. 267

Figure C-3: Distribution of error on thermocouple readings ................................... 268

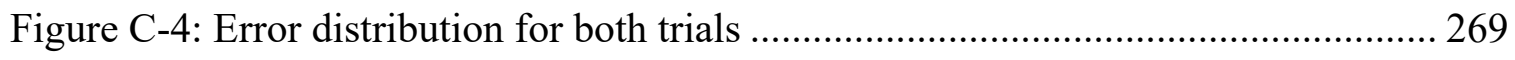

Figure F-1: TRNSYS model for calculating the baseline energy consumption using the

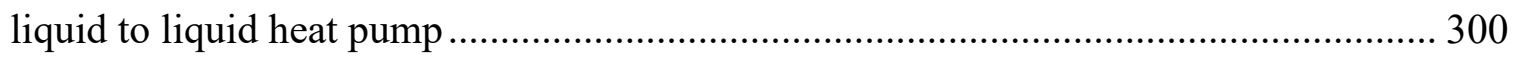

Figure F-2: TRNSYS model for the complete system using thermal storage and domestic

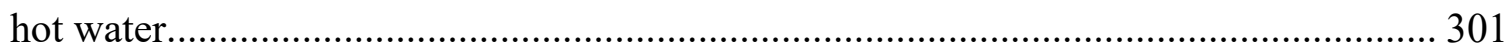

Figure F-3: TRNSYS model of the cold thermal storage, including the control

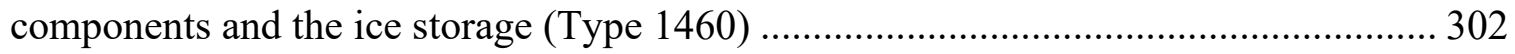
Figure F-4: TRNSYS model of the air handling unit, including the mixing of the return air from each zone and the splitting of the air to each of the three zones...................... 302 Figure F-5: TRNSYS model used to calculate the time energy is used and the total consumption, and whether it is peak, mid peak or off peak periods ............................ 303 Figure F-6: TRNSYS model of the domestic hot water system, using a preheat tank and

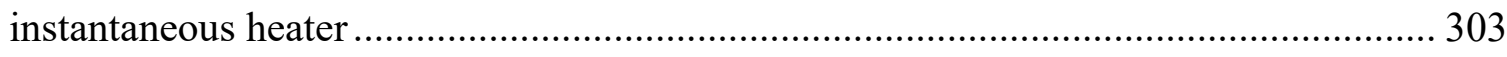




\section{Nomenclature}

\section{Symbol}

A

COP

$\mathrm{c}_{\mathrm{p}}$

D

e

E

g

$\mathrm{h}_{\mathrm{c}}$

$\mathrm{H}_{\mathrm{f}}$

IF

$\mathrm{k}$

L

$\dot{m}$

$\mathrm{m}$

$\mathrm{n}$

$\mathrm{Nu}$

q

r

$\mathrm{Ra}$

$\mathrm{Re}$

$\mathrm{t}$

$\mathrm{T}$
Description

Area

Coefficient of performance

Specific heat

Pipe Diameter

Electrical energy consumed

Energy Stored

Acceleration due to gravity

Convective Coefficient

Heat of formation

Percentage of tank that is ice

Thermal Conductivity

Length

Mass flow rate

mass

Node Number

Nusselt Number

Heat transferred

Radius

Rayleigh Number

Reynolds Number

Time

Temperature
Units

$\mathrm{m}^{2}$

$\mathrm{kJ} / \mathrm{kg}{ }^{\circ} \mathrm{C}$

$\mathrm{m}$

$\mathrm{kJ}$

$\mathrm{kJ}$

$\mathrm{m} / \mathrm{s}^{2}$

$\mathrm{W} /\left(\mathrm{m}^{2} \mathrm{~K}\right)$

$\mathrm{kJ} / \mathrm{kg}$

$\mathrm{W} /(\mathrm{mK})$

$\mathrm{m}$

$\mathrm{kg} / \mathrm{s}$

kg

kJ

$\mathrm{m}$

S

${ }^{\circ} \mathrm{C}$ 


$\begin{array}{ccc}\mathrm{V} & \text { Velocity } & \mathrm{m} / \mathrm{s} \\ \mathrm{V} & \text { Volume } & \mathrm{m}^{3} \\ \varepsilon & \text { Storage effectiveness } & \\ v & \text { Kinematic Viscosity } & \mathrm{m}^{2} / \mathrm{s} \\ \beta & \text { Volumetric Expansion } & 1 / \mathrm{K} \\ \alpha & \text { Thermal Diffusivity } & \mathrm{m}^{2} / \mathrm{s} \\ \rho & \text { Density } & \mathrm{kg} / \mathrm{m}^{3}\end{array}$

\section{Subscripts}

$\begin{array}{cc}\text { avg } & \text { Average } \\ \text { cooling } & \text { Space cooling provided } \\ \text { Heat_pump } & \text { Ice growth rate } \\ \text { heating } & \text { Value for the heat pump } \\ \text { i } & \text { Space heating provided } \\ \text { ice } & \text { Node indicator } \\ \text { load } & \text { Conditions at ice boundary } \\ \mathrm{m} & \text { Load side of the heat pump } \\ \text { max } & \text { Ice melting rate } \\ \text { net } & \text { Maximum value } \\ \text { o } & \text { Sum of growth and melting rates } \\ \text { out } & \text { Outer boundary } \\ \text { reak } & \text { Outlet conditions } \\ & \text { Energy used during peak periods } \\ & \text { Reference value }\end{array}$


stored

source

water
Energy being stored

Source side of the heat pump

Values for water 


\section{Chapter 1: Introduction}

\subsection{Motivation}

In 2017, space heating and cooling accounted for $61.6 \%$ of total residential secondary energy consumption, and $10.2 \%$ of all secondary energy consumption in Canada [1]. Although natural gas has an increasing share of Canadian space heating, particularly in Ontario and major urban centers, 39\% of Canadian households use electricity as the primary energy sources for space heating, while all households use electricity for space cooling [2]. On top of the significant energy consumption space heating and cooling of residential buildings are responsible for, these loads are primarily responsible for the peak consumption loads placed on the electrical grid [3].

This increased peak load has many detrimental effects on the utility grid, on the environment and to the consumer. The electrical grid must be sized in terms of both generation and transmission capacity to meet the annual peak load to ensure uninterrupted services throughout the year. As a result, a larger peak load requires the utility providers to increase their generation capacity and ensure their transmission capabilities can accommodate the peak electrical consumption. Increasing the generation and transmission capacity of the grid requires significant capital investment, which consequently causes an increase in utility costs [4]. Additionally, during peak loading periods, in many jurisdictions, including Ontario, the base electrical demand is met using low greenhouse gas emitting methods including nuclear and hydroelectricity, while peak loads must be met using greenhouse gases intensive methods that typically burn fossil fuels $[4,5]$. As a result, 
electricity generated and consumed during peak consumption periods produces more greenhouse gases per unit energy compared to non-peak periods.

To combat peak electrical consumption, utility providers are implementing financial incentives to consumers to move electrical consumption to off-peak periods. The most prominent of these is the introduction of time-of-use billing, in which different rates are charged per kilowatt-hour of electrical consumption based on when the electricity is consumed. Peak periods are defined by each utility provider or regulatory body and are dependent on the time of year and whether it is a heating or cooling dominated jurisdiction. As a result, a premium is paid for electricity consumed during peak periods compared to off-peak periods. Many jurisdictions have, or are in the process of switching to this billing method, including both Ontario and Nova Scotia, and some utility providers in the United States including Pacific Gas and Electricity in Northern California $[6,7,8]$. In addition to time-of-use billing, utility companies provided additional incentives to consumers to reduce peak loading over the years, including rebates for high performance chillers and for residential customers to switch to Wi-Fi enabled thermostats [9].

In addition to influencing occupant behavior through incentives and billing programs, mechanical equipment can be implemented that is designed to shift electrical consumption from peak to off-peak periods. These are most commonly implemented in large commercial and industrial applications, where thermal storage is paired with heating or cooling sources, which run during off-peak periods (typically overnight) and then store heating or cooling potential for use during peak period. Recently, a number of products have been introduced to the market to shift energy required for space heating from peak to off-peak periods. These work by heating up very dense bricks with a high thermal capacity 
to store heating potential, creating a shift between electrical consumption and heating demand. To date, very little focus has been placed on shifting residential electrical consumption required for space cooling to off-peak periods using thermal storage methods.

This thesis outlines the completed and proposed work on assessing the potential for cost, energy, and greenhouse gas reduction possible by shifting electrical consumption from peak to off-peak periods using thermal storage paired with a liquid to liquid heat pump. This system will charge the thermal storage during the overnight, off-peak period, for use when cooling is required during peak electrical consumption periods. To determine the overall feasibility and optimize these systems, the primary focus of the work is to develop small-scale, compact cold thermal storage systems that could be easily integrated into residential applications, and provide adequate cooling to offset close to $100 \%$ of the daily peak and mid-peak cooling load. To do this, sensible thermal storage systems using both water and a water/glycol solution have been evaluated, and small-scale cold thermal storage systems using ice as the storage medium are being developed. Although cooling loads and cold thermal storage will be the primary focus of this work, heating and domestic hot water loads will also be considered and factored into the design and analysis.

\subsection{Proposed System}

To shift peak residential electrical loads, a system is proposed that incorporates both hot and cold thermal storage systems, connected with a liquid to liquid heat pump. During the heating period, low grade heat is inputted into the cold thermal storage system, extracted by the heat pump, and deposited into the hot thermal storage system, at a much higher temperature. The space heating and domestic hot water load is then met using the stored thermal energy. The low grade heat input can be from any number of sources, 
including using a ground loop, solar thermal collectors, or a district or central heating loop [10]. These sources of energy would be outside the scope of the work and would provide an input to the cold side of the heat pump. All sources would act in the same way, where cold fluid is drawn from the heat pump of cold thermal storage system, heat added to the fluid, be it from the heat source and then returned to the heat pump or the thermal storage. During the cooling season, energy is extracted from the cold thermal storage tank, and then deposited into the hot thermal storage until the set-point is reached, providing the required energy for domestic hot water, at which point, the heat is then dumped outside, or stored using a seasonal thermal storage system [11]. The cooling load is then met using the cooling potential stored within the cold thermal storage tank. A high-level schematic of the proposed system is shown in Figure 1-1.

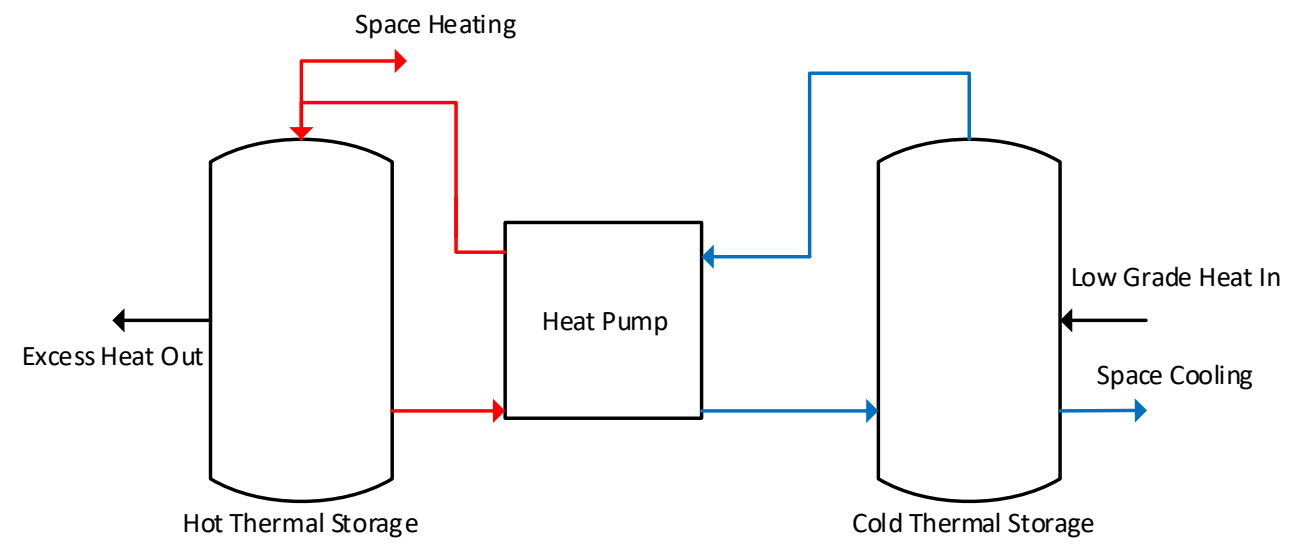

Figure 1-1: High level schematic of the proposed system with the heat pump connected to a hot and cold thermal storage system

The preliminary configuration of this system has been derived from a solar-assisted heat pump system used for space heating and cooling of Team Ontario's entry into the 2013 U.S. Department of Energy's Solar Decathlon [12]. When originally designed, the system used energy from four flat plate solar thermal collectors and was designed and optimized to minimize total annual energy consumption needed to meet the space heating, 
cooling and domestic hot water loads. This project extensively examined the relation between the heat pump, hot thermal storage and the heating loads but did not consider the cold thermal storage. The system has been reconfigured to examine the relation between the heat pump and the cold thermal storage, as well as optimizing the cold thermal storage.

\subsubsection{Heat Pumps}

The central component of the proposed research is a liquid to liquid heat pump. A heat pump is a mechanical device that compresses a refrigerant between an evaporator, where heat is extracted at a lower temperature, and a condenser, where heat is released at a much higher temperature [13]. This allows energy to be upgraded to a higher temperature for use in many applications. Heat pumps use the phase changes of the refrigerant to upgrade large quantities of heat in comparison to the electrical energy input into the compressor. As a result, heat pumps can provide in excess of five times the heat compared to the electrical input (coefficient of performance), making heat pumps one of the most efficient methods for meeting space heating, cooling and domestic hot water loads.

\subsubsection{Thermal Storage}

The use of two thermal storage systems is pivotal to the proposed system. Thermal storage is a system that stores heat or cooling potential from the time it is generated to the time at which the use is required [14]. Thermal storage systems can be broken down into two main categories. The first is diurnal which has a charge/discharge period in the order of a couple of hours to a couple of days. This type of system is most commonly used to store energy for use later in the day, and is extensively used with solar thermal heating and hot water systems [15]. Solar energy is readily available during the daytime periods, however thermal storage is required to meet heating and hot water demands overnight. The 
second main category of thermal storage is seasonal storage, which has an annual charge/discharge cycle. These systems are much larger than diurnal systems, typically with volumes 2-4 orders of magnitude greater than diurnal storage, and are used to meet the seasonal mismatch of when energy is available and when energy demand is greatest [15]. These include borehole thermal energy storage systems, where energy is inputted into the ground during the summer either as waste heat from cooling systems or from solar thermal systems, and then extracted during the heating season and used for space heating.

Thermal storage has been most commonly used to store thermal energy that can be used at a later time for heating applications, however as demand and energy use increases for space cooling, thermal storage systems are being implemented to store cooling potential. In addition to categorizing thermal storage systems as either diurnal or seasonal, and as hot or cold, the storage medium can also be used to define or characterize a thermal storage system. Three main types of thermal storage mediums can be implemented. The first and most common is sensible thermal storage, where energy is stored via a sensible change in temperature of a storage material, most commonly water. Although sensible storages are the simplest, cheapest and most common, they tend to have a lower storage density, which is the quantity of energy that can be stored either per volume or per mass.

For applications where higher densities (and consequently a smaller system) are required, the use of phase change materials can be implemented. Specific materials are selected for the temperature of interest, where the melting point is the desired storage/supply temperature, and the energy is stored by melting the storage medium, and when energy is required, the material is solidified, releasing heat. This process can also be used in reverse as a cold thermal storage system. The final type of storage medium is 
chemical storage, where chemical reactions are used to store energy. Typically hydrates are used, where the separation of the water molecules from the host chemical requires heat input, which is subsequently stored as separated substances, which can then be released by allowing the water to be recombined with the host substance, which is exothermic, releasing heat that can be used.

To date most thermal storage systems are used to meet a temporal mismatch between when an energy source is available and when energy use is required, however, systems can also be implemented to shift when energy is used, as a method for reducing or removing peak energy consumption. These systems not only reduce the peak load placed on the utility grid, but also can take advantage of lower energy costs during off-peak periods, providing energy cost savings to the building owner.

\subsection{Research Objectives}

The overall objective of the completed and planned work outlined in this thesis is to develop a system for use in residential applications, having a small footprint and being able to off-set a significant portion of the peak loads on an annual basis. To meet this overall objective a number of sub-objectives have been developed.

- Create a detailed heat pump performance map at low temperatures and flow rates to determine the relationship between the heat pump and thermal storage performance.

- Validate current TRNSYS storage tank models which have historically been used to simulate hot thermal storage for use as a sensible cold thermal storage system.

- Develop a small-scale ice storage system for use in daily demand side management.

- Experimentally determined ice growth rates with different flows and pipe 
configurations using a small-scale experimental test rig.

- Design a small-scale ice storage system capable of off-setting one day's worth of peak cooling loads.

- Develop a computer model of the proposed small-scale ice storage system.

- Develop a whole house models and perform annual simulations to determine influence of the proposed system on peak electrical demand reduction.

- Examine the economic potential for this system over its proposed life-span.

- Determine the potential for off setting peak loads for multiple locations across North America.

\subsection{Outline of Thesis}

The remainder of this thesis is broken down as follows:

- Chapter 2 presents a detailed literature review pertinent to this study, including the energy landscape in Canada, heat pumps, thermal storage with an emphasis on cold storage and performance metrics. The gaps in literature and how the current work fits within literature and the current state of knowledge will be discussed.

- Chapter 3 presents the experimental set-ups used through this work, including for the testing of heat pump performance, interaction with thermal storage systems and the performance of an ice thermal storage system.

- Chapter 4 presents the component modelling, including the validation of the heat pump models and the sensible thermal storage models, as well as the development and validation of the ice storage model.

- Chapter 5 presents the development of the house model used in the study, how the heating and cooling system is modelled as a whole, and the interaction between the 
heat pump and the thermal storage systems, including impact of flow rates on the overall performance.

- Chapter 6 presents the annual simulations and performance of the system, as additional components and building loads are included within the study, and the potential for off-setting peak loads, and the economic impacts for Ottawa are determined.

- Chapter 7 presents an analysis and discussion on the potential for off setting peak loads economically, including the examination of different locations across North America, and an analysis of different rate structures and life cycle costs.

- Chapter 8 presents the conclusions of this work and a discussion on future work that could build off of this study.

\subsection{Contributions to the Field}

This project will make a significant contribution to the field, as little to no work has been conducted on residential demand side management using heat pumps and thermal storage. This system has the potential to save both the consumer and the utility providers money. Through this Ph.D. project, the following specific contributions to the state of knowledge in the field are expected:

- Validate existing TRNSYS tank models for stratified, low temperature applications.

- Determine the potential and required tank sizes to offset daily peak heating and cooling loads using sensible thermal storage.

- Develop a detailed performance map for a medium temperature chiller under steady state and transient conditions for low flow rates. 
- Develop a model for a small-scale ice thermal storage system using heat transfer rates, and validated using experimentally obtained data.

- Determine the optimal configuration to provide the best economic returns based on a house located in Ottawa

- Determine the potential for the system in multiple locations across North America with differing rate structures and climactic conditions

- Determine the lifecycle cost and economic potential for the proposed system through the offsetting of peak loads

Through this work, four peer reviewed conference papers have been published, along with a book chapter on sensible thermal storage. Currently, two journal articles on the modelling and comparison of ice storage and sensible storage systems and on the potential and life cycle costs of undertaking demand side management in residential settings using a heat pump and thermal storage are being prepared. 


\section{Chapter 2: Background and Literature Review}

This chapter presents current literature pertinent to the overall research project. This includes examining the electrical landscape in Canada, with a detailed focus on the energy landscape in Ontario, which will be predominantly used for this work. Current methods being employed for demand side management are presented, including time-of-use billing and incentive programs employed by major utility providers. A background on residential heat pumps and thermal storage will be presented with a focus on the integration of these technologies into a cohesive system. A detailed review will be conducted on the current state of knowledge and technology for small-scale cold thermal storage systems. The metrics used to assess all aspects of heat pumps, thermal storage, and residential energy use will be listed. Finally, the current gaps in literature are presented, with these gaps forming the basis of the proposed thesis work.

\subsection{Shifting Electrical Consumption from Peak to Off-Peak Periods}

A thorough analysis of the current energy landscape in Ontario was conducted and is presented in Appendix A. From this, it can be seen that there are significant benefits of shifting energy consumption from peak periods to off-peak periods. From an economic standpoint, every kilowatt-hour of electricity that is shifted from peak to off-peak periods currently saves the consumer 9.3 cents. This number will increase over time as historically the peak electrical rate has increased at a greater rate than the off-peak rate, with off-peak rates increasing 57\% since May 2010, while peak rates have increased $77 \%$ during the same period [16]. The increasing peak load on the electrical grid is a driver for increased utility prices as it increases the required generating and transmission capacity on the grid. This is captured in the global adjustment component of the utility price, which covers the 
maintenance of the existing infrastructure, as well as recuperating the capital costs of new transmission and generating capacity. Although all of these numbers seem very small, they are on a per kilowatt-hour basis, and when extrapolated over the course of a year, can lead to cost savings in the hundreds of dollars. In addition to the cost savings associated with shifting the time of electricity use, significant environmental benefits can be realized as well. As calculated in Appendix A, for every kilowatt-hour of electricity shifted from peak to off-peak periods during the winter months, a reduction of $8.5 \mathrm{~g}$ of $\mathrm{CO}_{2}$ emissions can be realized, while during the summer months, this reduction increases to $21 \mathrm{~g}$ of $\mathrm{CO}_{2}$. If widespread implementation of load shifting systems were to occur in Ontario, the reduction in harmful GHG emissions could be significant.

\subsection{Utility Based Demand Side Management}

Demand side management (DSM) is the planning, implementation, and monitoring of activities initiated by a utility provider to influence the customer's electricity use habits [17]. This includes changes in a consumer's time pattern and quantity of electrical consumption [17]. Although this thesis will focus on DSM in terms of electrical consumption, non-electrical utility providers (most notably natural gas providers) create programs focused on customer consumption habits as well. Based on this definition, any program that provides an incentive for the consumer to change their consumption habits, including reducing overall consumption and/or shifting when they consume electricity would be considered DSM. Ontario has had a number of DSM policies in place in recent years, including providing rebates on the purchase of new, energy efficient HVAC equipment and on Energy Star LED and CFL light bulbs [9]. In addition to these rebates, the Ontario government has implemented a program called the Peaksaver Plus, which uses 
a special WiFi enabled thermostat, water heater controller or pool pump and heater control, allowing the utility provider to take control of these high demand devices during summer periods of peak electrical consumption [18]. The utility provider is then able to strategically cycle homeowner's devices on and off at set time periods, smoothing the overall electrical consumption of the grid.

On top of incentives offered to consumers for the installation of equipment and lighting that will reduce the overall electrical consumption, the single greatest DSM program that has been implemented in Ontario is the implementation of time-of-use billing practices. This billing practice incentivizes the use of off-peak electricity, while charging a premium for energy consumed during peak periods. The change in price between offpeak and peak periods is an incentive to get consumers to change their consumption habits, shifting their electrical consumption to off-peak periods. The cost benefits and greenhouse gas reductions for the consumer as a result of shifting electrical consumption has been extensively discussed in Appendix A. On top of the benefits for the consumer, this shift of electrical consumption from peak to off-peak periods is of critical importance and interest to electrical utility providers. The electrical grid and generating capacity must be sized to meet the anticipated peak electrical load for any given year, and as a result, the generating and transmission capacity for a region is dependent on the anticipated peak consumption. Consequently, the reduction of peak load through DSM can have significant capital savings for the utility provider and is the driving factor behind the incentives provided to the customer.

Almost all DSM programs and incentives target the reduction in overall energy consumption through upgrades to more energy efficient equipment, or to change the 
consumer's behavior shifting energy use to off-peak periods. To successfully achieve the target of the DSM program (reduced consumption or shifted consumption), the consumer is relied upon to change their day to day behaviors, changing their electrical consumption patterns, or relinquished control of their systems, possibly impacting their comfort and habits. To date, very few DSM measures implemented are autonomous, and having no impact on the everyday lives of the consumers.

\subsubsection{Smart Grids}

A smart grid is an electrical grid that distributes not only electricity, but simultaneously transmits data between consumer and generation sources. Smart grids are used to allow for the improved alignment between power generation and consumption [19]. The current state and source of generation and level of demand is provided to the consumer, while simultaneously data is transmitted to the generating station on overall demand to better manage the source and total generation. The primary focus of research in recent years has been to utilize the data provided from the grid to control variable electrical loads, including large appliances (most notably the dryer) and HVAC equipment, using what is often referred to as dynamic demand response. This can take either two forms. The first is to trigger large energy consumers when renewable generating capacity is at its greatest, with the goal of reducing the energy consumption when demand is met using fossil fuels, as outlined in the study by Pina et al. [20]. This work is applicable where there is a large renewable penetration into the electrical grid from variable sources (solar and wind primarily), with the remainder of electrical generation from fossil fuels-based sources. Although this type of system works with smaller electrical grids (the study focused on the Azores, small islands located in the east Atlantic), to date, a smart grid concept has not 
been developed and implemented into a grid the size of Ontario. Additionally, variable renewable electricity generation is responsible for less than $6 \%$ of the total generation in Ontario.

On the opposite end of the spectrum, the second method of dynamic demand response is to reduce energy consumption during peak periods, as presented by Fernandes et al. [21]. During peak periods, the information for the grid can be used by an energy management system within the house, allowing decisions to be made that reduce electrical consumption during these periods. This includes shutting down energy intensive devices like large appliances or alter the heating or cooling set-point of the thermostat to reduce energy consumption. The conversion to a smart grid in Ontario would be extremely costly and difficult, however energy management systems using the peak and off-peak times set out in Ontario's time-of-use billing practices can lead to significant reductions in peak loading.

In addition to looking at just real time demand response, extensive research is currently being conducted on predicting future consumption. A study by Logenthiran et al. [22] looked at predicting energy consumption for the next $24 \mathrm{hr}$ period to make informed decisions on energy consumption. Using the predicted energy consumption and building response, decisions were made within the energy management system to smooth total electrical consumption, in residential, commercial and industrial applications, with substantial savings realized and a significant reduction in peak loads. To implement responsive or predictive DSM systems, algorithms that factor in time-of-use, electrical costs and total energy consumption must be developed and implemented. Research has been conducted on developing and optimize these algorithms, and are outlined in studies 
by Logenthiran et al. [23], by Lopez et al. [24], and Thomas et al. [25] which also factor in the relationship between the smart-grid, demand side management and the charging of electric vehicles. Although smart grid integration with energy management systems can greatly reduce the peak load on the electrical grid, with all studies on the matter seeing a significant reduction in peak loads, they will typically be at the expense of occupant comfort, or requiring active input or change in habits of the occupant.

\subsection{Consumer Based Demand Side Management}

In addition to the DSM methods currently being employed by utilities, extensive research is currently being undertaken to develop alternate strategies. This research focuses on both the overall reduction in electrical consumption, and of particular interest to this work, methods for shifting electrical consumption. This section outlines recent work completed using smart grids, electric heating with thermal storage, changes to building design and chillers with cold thermal storage. The difference in consumer and utility-based DSM is who ultimately has decision making authority on the control of the system and implement when measures are taken. A review of residential demand side management approaches by Esther and Kumar [26], and found that few had meaningful interaction in the grid, and could be achieved through building design, occupant action and mechanical systems.

\subsubsection{Passive Building Design}

Most DSM strategies utilize active systems (mechanical systems that required energy input to provide space heating or cooling), however passive systems (systems that do not require continuous electrical input to function) can be equally as effective in shifting electrical consumption. This can be accomplished through the building design, including 
the strategic placement of windows, optimizing when heat gains occur while reducing unwanted heat gains, and increased insulation levels. Although building design can have a significant impact on electrical consumption for space heating and cooling, it can only achieve a certain level of peak shaving.

To achieve significant peak shaving using passive methods, advanced measures are required, and a number of studies have been conducted in recent years. To achieve a passive control of demand side management, the designed integration of thermal mass into the building is required. This thermal mass is able to passively collect solar heat during the daytime and release it overnight during the heating season, or cool down overnight, and absorb excess heat during the daytime period. This can be achieved using both sensible (high mass construction) or latent thermal storage (phase change materials) can be utilized. To achieve substantial results, a significant amount of thermal storage is required due to the small temperature differences available in passive applications, and therefore applications that have used phase change materials have had a larger impact on controlling when energy is used.

A study by Zhang et al. [27] showed that the integration of phase change material into the floor and/or ceiling of a house can smooth electricity consumption, storing solar thermal energy from the day, releasing the heat overnight. The reverse occurs during the cooling season, with cooler night-time air solidifying the phase change material, releasing the cooling potential through the day through the melting of the material. A second study conducted by Qureshi et al. [28] integrated phase change materials into gypsum board for use in an office building. Two identical spaces, one with the phase change material and one without were compared, with the space utilizing the phase change material saw a decrease 
in the amount of time the heating system was on, a shift towards lower demand times, and a reduction in excess of 50\% in total energy consumption for space heating over the days tested. Although passive systems, most commonly using building integrated phase change materials, have been proven to reduce peak load, there is no control over passive systems. As such, the benefits are hard to estimate, and maximum benefits of the infrastructure cannot be achieved. As a result, the use of active systems provides complete control to the occupant, limited impact on the occupant and have the potential to provide greater reduction in peak electrical consumption.

\subsubsection{Electric Heating with Thermal Storage}

A more decentralized, emerging method for DSM is the use of electric resistance heaters in conjunction with thermal storage. These can be used either for space heating or domestic hot water heating. A number of studies have been conducted on the development of control strategies utilized by hot water heaters, including by Paull et al. [29], Nehrir et al. [30] and by Chen et al. [31]. Each of these studies propose new control strategies for engaging and disengaging the heating elements, with a common goal of reducing the peaks associated with water heating. These include predicting when hot water demand is required, and starting to preheat the water before demand occurs, or by changing the water set-point based on the time of day, increasing the set-point during off-peak times, storing additional energy for use during peak periods. This is of particular interest during the overnight period, when electricity consumption is at its lowest, and directly precedes the period of greatest hot water use which typically occurs first thing in the morning.

In addition to hot water control strategies for demand side management, electric heaters can be paired with thermal storage to allow electrical consumption and spacing 
heating to be time independent. That allows the electrical consumption required for space heating to take place during off-peak periods, while the space heating from this electrical consumption can be realized at a later time, typically from peak to off-peak periods. These systems are currently commercially available and are becoming popular for consumers using electric resistance heating in jurisdictions with time-of-use billing [32, 33]. A number of studies have been conducted, including by Molina et al. [34] and by Arteconi et al. [35], proving these devices can successfully shift a significant portion of electrical consumption required for space heating to off-peak periods. These devices work by heating up high density bricks, heated during off-peak periods to very high temperatures, and then released slowly when heating is required, at a variable rate based on the heat required at the specific time. These systems are increasingly being incentivized by utility providers, with Nova Scotia Power leading the way. For a consumer to be permitted to change their rates to timeof-use billings, Nova Scotia Power requires the installation of an electric based heating system with thermal storage with advanced control. Through this program, consumers using electrical heat are able to realize significant cost savings shifting their consumption from peak to off-peak periods. These systems have shown, both through academic study and real applications, that a significant portion of peak energy consumption required for space and domestic hot water heating within the residential sector can be shifted to offpeak periods. The downside to these systems however, is that electric resistance heating is required, which is a poor use of high grade, electrical energy, and with a coefficient of 
performance of one, has a lower electrical performance when compared to other electric heating systems, most notably heat pumps.

\subsubsection{Heat Pumps with Thermal Storage}

To improve the overall energy performance of an electric resistance heating system, a heat pump can be used to replace the resistance heater. Heat pumps use the compression and expansion of a refrigerant to extract heat from a source, upgrade to a higher temperature and provide it to the source. Heat pumps are able to obtain an annual average heating coefficient of performance in excess of 4, meaning they supply 4 units of heat for every unit of electrical input, or when compared to resistance heaters, use a quarter of the electricity to provide the same amount of heat [36]. Additionally, a heat pump system can provide not only heating, but space cooling, and in some cases domestic hot water. The use of a heat pump alone can be viewed as a DSM method, as they provide a significant reduction in overall energy consumption, however a heat pump alone does not provide any control over when electricity is consumed [37].

For heat pumps to be used to provide load shifting capabilities, thermal storage must be used in conjunction with the heat pump. This allows the heat pump to run during off-peak periods, storing up heating or cooling potential, and allows this potential to be realized when required. This can be a passive thermal storage system, in which thermal storage is distributed throughout the dwelling and once charged, will passively provide space heating or cooling. The most common method for this is to use the mass of the

building itself as thermal storage, as discussed in studies by Ellerbrok [37], Hedegaard and Balyk [38] and Masy et al. [39]. In each of these studies, the dwelling incorporated concrete structural components, most commonly the floor, and the heat pumps are used to heat the 
thermal mass either directly (e.g. embedded radiant pipe) or indirectly (through heating the air in the space). All three studies found that like the passive thermal mass not coupled to heat pumps, the integration of passive thermal mass in conjunction with heat pumps reduced the amount of time the heating or cooling system is on. It was also found that when the control system is optimized, the thermal mass working in conjunction with the heat pump can shift electrical consumption from peak periods. The downside as found in these studies was the release rates of heating or cooling is only controlled by the temperature difference between the thermal mass and the space. This prohibits obtaining the maximum benefit of releasing the stored potential when electrical consumption is at its peak. This draw back can be alleviated by the use of active thermal storage systems, which allow for greater control of both when energy is stored, but also when the stored potential is utilized.

As heat pumps must use a heat transfer fluid to provide space heating or cooling (most commonly air or water), this heat transfer fluid can be used to directly charge thermal storage systems, which can then be used to meet the space conditioning demands. This is typically achieved in one of two configurations. The first is the heat transfer fluid is also the thermal storage medium, where a reservoir or tank of the fluid is heated, and the sensible increase in the fluid temperature provides the thermal storage. This is most commonly achieved using water, as it is a cheap material with a high density and heat capacity but can also be achieved using glycol solutions. The second method is to use the heat transfer fluid as a mechanism to transfer the heat from the heat pump to the storage 
medium, which can use sensible, latent or chemical storage and can use either water or air as the heat transfer fluid.

Different configurations with active thermal storage systems have been studied using both hot thermal storage systems for space heating as well as using cold thermal storage systems for space cooling. Hirmiz et al. [40] studied the integration of phase change materials with a heat pump to shift the electrical load needed to meet the cooling demand. The phase change material had a melting point of $10^{\circ} \mathrm{C}$ and was able to reduce the volume three-fold compared to a chilled water system. Through this system, peak loads could sustain for 2 to 6 hours depending on the volume used. Moreno et al. [41] compared using a cold water storage tank and an encapsulated phase change material with a melting point of $10^{\circ} \mathrm{C}$. Mixed results were observed, with a $35 \%$ increase in the storage density with the phase change materials, however, the charging time increased $455 \%$ and the charge and discharge efficiency saw significant degradation when going from phase change to sensible. A second study conducted by Real et al. [42] paired a water to water heat pump with a cold thermal storage system utilizing a phase change material with a melting temperature of again $10^{\circ} \mathrm{C}$. In this case, the thermal storage was used to temper the conditions of the inlet of the heat pump, by increasing the supply temperature for the source side of the heat pump during heating, and provides a lower heat sink during space cooling operations. This allowed an increase in heat pump performance on an annual basis, however this system had limited impact on time electricity is used as result of the control strategy being implemented. A third study from Arteconi et al. [43] looked at the implementing a heat pump coupled with hot thermal storage and a two different water based distributions systems in radiators and radiant floors. A stratified hot storage tank was 
used, with the control strategy turning off the heat pump between $4 \mathrm{pm}$ and $7 \mathrm{pm}$ (peak periods in Ireland - the location of study). This system was found to shift the energy consumption for space heating during the peak period, and when Ireland's time-of-use billing practices are implemented, where peak electricity is almost three times the cost of off-peak costs, this strategy was shown to reduce utility bills for the homeowner.

These studies have all shown potential for heat pumps to be paired with thermal storage systems to achieve demand side management, in shifting the space conditioning loads from peak to off-peak periods. Some of the studies have discussed the potential for using the proposed systems to offset both heating and cooling loads, however all studies found have looked at only the heating or the cooling season, with no comprehensive studies on combined systems. This limitation could be as a result that the previously completed work has been primarily conducted in areas that either have limited or no heating load (Mediterranean) or limited cooling load in Northern Ireland. These studies also showed limited impact on the offsetting of cooling loads as it is very difficult to store cooling potential, as a very large temperature difference is hard to obtain. Both cold thermal storage systems studied utilized phase changes materials in the form of different types of waxes with a melting point of $10^{\circ} \mathrm{C}$, allowing for additional cooling potential to be stored when compared to sensible storage. Although more energy was stored, the effectiveness of converting cooling potential from the heat pump to stored cooling potential was significantly lower than when compared to sensible thermal storage. This resulted in an 
increase in the required electrical input to meet the building's cooling loads within the studies.

No completed studies could be found using heat pumps and thermal storage for demand side management during the cooling season with cold thermal storage temperatures below $10^{\circ} \mathrm{C}$. It is believed that is because the performance of standard heat pumps and air conditioning systems degrade significantly at evaporator temperatures below $10^{\circ} \mathrm{C}$. This phenomenon is discussed by Dincer and Rosen [44], stating that both the coefficient of performance and cooling capacity of the chiller experience a significant degradation in performance, with the capacity decreasing $56 \%$ and the COP decreasing $70 \%$. This shows that to utilize thermal storages that requires lower storage temperatures (including ice storage), alternate compressor configurations or technologies must be utilized.

\subsection{Heat Pumps}

Heat pumps use a vapour compression cycle to transfer and upgrade energy from a colder source to a hotter sink through the use of mechanical work. To transfer heat from the source to the sink, a refrigerant is circulated through the heat pump. Energy is extracted from the source, causing the refrigerant to evaporate, it is then brought to a higher pressure using a compressor, and then the energy is transferred to the heat sink through the refrigerant condensation [13]. A heat pump can be used for heating or cooling of a space, and is used to in refrigerators to provide cooling, as well as air conditioners. In a building 
sense, a system is commonly referred to as a heat pump when it provides space heating, even though an air conditioner is, by definition, a heat pump as well.

Within the residential market, heat pumps are used for space heating and cooling, as well as domestic hot water heating, and are typically categorized by the source of lowgrade heat. Traditionally, heat pumps have extracted energy from outdoor air (air-source heat pump) or from the ground using boreholes and a liquid-source heat pump, however in recent years, advances have been made using heat from solar thermal systems as the source energy. In areas with district heating systems or in multi-unit complexes (condominiums), heat pumps are being used to extract energy from a central loop and upgraded to meet the demands of the individual unit.

Historically, in cold climates, space heating loads when outdoor temperatures were below $-5^{\circ} \mathrm{C}$ could only be met using a ground source heat pump, as heat could not be extracted from the cold ambient air using an air source heat pump. In recent years, extensive research has been conducted on heat pump technology to improve performance at colder source temperatures. This work has primarily focused on the development and optimization of multi-stage compression systems. These systems can take a number of different configurations, based on the required temperatures, but can be broken into two general categories [13]. The first is a cascade cycle, in which two or more refrigeration cycles are placed in series, where the heat sink of the first acts as heat source of the second. Each cycle in the series typically uses a different type of refrigerant, allowing for different temperature ranges in each cycle. The second configuration uses multi-stage compression with intercooling. This configuration uses two compressors and a flash tank or intermediate heat exchanger to cool the refrigerant between the first and second compressor. This 
configuration requires less energy to move the refrigerant to the required pressure and temperature when compared to compressing the refrigerant in a single step [13].

These advanced cycles have recently been studied to determine their applicability for air source heat pumps in the residential sector. The use of a multi-stage compression system allows for improved performance at low evaporator (source) temperatures, and as such could improve the performance of air source heat pumps in cold climates. Wang et al. [45] studied a system that utilized a flash tank to inject low temperature refrigerant vapour into an intermediate stage of the compressor. Using this configuration, observed cooling is in-line with a standard system, however at an evaporator temperature of $-17.6^{\circ} \mathrm{C}$, the multistage system has a capacity $33 \%$ higher, and a COP $23 \%$ higher than the base system at those temperatures. A similar study by Bertsch and Groll [46] used a two-stage heat pump with R-410A for cold climate applications. Heating of both air and water was tested, but only air was used for source energy. No experimental comparison to a traditional system of similar configuration was provided, however heating COPs of 2.1 were observed at evaporator temperatures of $-30^{\circ} \mathrm{C}$. This extended the range of source temperatures the heat pump could operate with, and an improvement over a simulated single stage compressor was reported once the evaporator temperature dropped below $0^{\circ} \mathrm{C}$. A third study by Kim et al. [47] studied a cascade cycle that used R410A in the lower temperature cycle and R134a in the higher temperature cycle. The experimental results show a COP improvement at evaporator temperatures of $-10^{\circ} \mathrm{C}$, however comparing the results from this cascade 
system to the previously discussed multi-stage systems with intercooling, the cascade systems have a much lower performance.

All of the literature available on using multi-stage compressors examined their use for space heating with a low, outdoor source temperature. That being said, the results from these studies also show that there is, in theory, potential to use a multi-stage compressor system to obtain increased performance for cooling of a thermal storage system at lower temperatures, while still achieving high load temperatures during the heating season. Through a search of scientific research and commercially available systems, all multi-stage compressor systems that could be found all use air for the source energy and then heat distributed using either air or water. As such, if a multi-stage compressor system is desired for use in a liquid to liquid system, a custom designed and fabricated system would be required based on the required temperature and capacity requirements for the application.

\subsection{Thermal Storage}

Thermal storage is the storage of thermal energy (either heating or cooling potential) during periods of energy abundance for use at a later time when the energy available is at a deficit in comparison to supply. Thermal storage can take many sizes, configurations, storage mediums and applications, and according to Pinel et al. [14] thermal storages are classified by storage mechanism (sensible, latent or chemical) and storage concept (active or passive). Within this research project, the focus is on pairing active 
thermal storages using sensible and latent thermal storage mechanisms with a liquid to liquid heat pump.

\subsubsection{Sensible Thermal Storage - Stratified Storage Tanks}

The most common thermal storage method is the use of heated water tanks. In this case, the thermal storage is achieved through the increase in temperature of the water. These systems owe their popularity to their low cost (predominantly as a result of water having little cost) and the high thermal capacity of water. To increase the effectiveness of sensible thermal storage systems, past research projects have focused on the promotion of stratification within the storage tank (or in some cases where multiple tanks were used, stratification through the system). Tank stratification is the existence of a temperature gradient through the height of the tank, allowing for the separation of the fluid by temperature zones, with the hottest zone at the top of the tank and the coldest zone at the bottom of the tank [48]. The use of stratified thermal storage systems have been extensively studied by, among many more, Cruickshank and Harrison [49], Dickinson and Cruickshank [50], and Lightstone, Raithby, and Hollands [51]. All of these studies showed the effectiveness of using stratified thermal storage tanks when paired with solar thermal systems, however many of the benefits observed when storing thermal energy from storage systems could be applied to a thermal storage connected to a heat pump. The drawback to utilizing a heat pump with thermal storage is as more energy is stored, the average tank temperature increases. If the tank is well mixed (constant temperature throughout), as the average tank temperature increases, the entering temperature into the heat pump increases. This causes a degradation in heat pump performance, as the lower the entering load temperature, the better the heat pump performance. If a stratified thermal storage tank is 
used, as the amount of energy being stored increases, the entering water temperature remains constant, allowing the heat pump performance to remain constant.

Although the use of water storage tanks have been extensively used and studied for hot thermal storage, the study of these tanks for use in cold thermal storage applications is very limited when compared to hot thermal storage. Cold thermal storage tanks have been studied both experimentally and through simulation. The fundamentals of stratified cold thermal storage were first studied by Nelson, Balakrishnan and Murthy [52] experimentally evaluated the stratification within a cold thermal storage tank, varying the aspect ratio, flow rate, inlet temperature and tank insulation. This study found that the aspect ratio is a critical parameter, and to increase charge and discharge efficiency and it is determined that a lower flow rate and higher temperature difference should be employed when possible. A second experimental study by Karim [53] which studied a $1 \mathrm{~m}^{3}$ cylindrical storage with a custom designed diffuser to promote stratification. The design of this diffuser was also evaluated as part of this study. It was determined that the thermal efficiency of the cold storage was as high as $90 \%$. Most of the loss of cooling potential is the heat transfer through the thermocline, which widens through the charge cycle. Both of these fundamental studies showed the potential for stratified cold thermal storage tanks, however they do not examine actual applications.

No studies were found that examined installed cold stratified thermal storage tanks for residential applications, however a couple were found for larger applications. A study of a system in Austin, Texas by Zhang et al. [54] containing a 13,249 $\mathrm{m}^{3}$ stratified cold storage tank. This system capitalized on the time-of-use billing present in Austin, Texas, and saved the operators almost $\$ 1$ million annually and a significant reduction in electrical 
consumption during peak periods was observed. A similar study by Sebzali, Ameer and Hussain [55] on a proposed system in Kuwait that was simulated to determine the potential for reducing peak electrical consumption. A "large" storage tank is implemented into a $3180 \mathrm{~m}^{2}$ building and stores water at temperatures between $4^{\circ} \mathrm{C}$ and $6.7^{\circ} \mathrm{C}$. This system was shown through simulation to reduce daily peak power demand for space cooling by $37 \%-88 \%$ and reduce overall annual energy consumption by $4.5 \%-6.7 \%$. Although both of these studies focused on systems that are much larger than those which could be used in residential applications, they do prove the potential benefits of using a stratified storage tank with a chiller or heat pump for demand side management and peak electrical reduction.

\subsubsection{Modelling Stratified Storage Tanks}

An important component within the proposed system are the stratified storage tanks. The tanks must be accurately modelled to predict the performance of the system. At a fundamental level, thermal storage tanks can be modelled with a high degree of accuracy using three-dimensional computational fluid dynamics. A number of studies have been conducted on the performance and stratification within thermal storage tanks utilizing three dimensional CFD, including by Nizami et al. [56], Consul et al. [57], Shah and Furbo [58],and Yaici et al. [59], among many others. All of these studies showed excellent results in terms of predicting the temperature distribution within the storage tank and the storage performance of the system, however the models were very computationally intensive, requiring long calculation times or the use of computing clusters. The use of CFD simulations is best suited for short time periods or to study tank geometries, however, as a result of their computational complexities, are difficult to use when studying the 
performance of thermal storage tanks over greater periods of time, e.g. an annual performance simulation.

To decrease the computational time requires a number of assumptions to be made to simplify the system and allow for analytical equations to be derived to predict the temperature distribution within a storage tank. The primary assumption that is made is that a cylindrical storage tank can be modelled in only one-dimension, along the axial direction. This assumption has been experimentally evaluated by Cruickshank [48] and was found that within a stratified storage tank, there is virtually no temperature change in the radial direction. To model the temperature distribution within a tank, the tank is divided up into $\mathrm{N}$ number of constant volume segments, commonly referred to as nodes. Each of these nodes is considered perfectly mixed and at a constant temperature, and an energy balance is conducted over each node, factoring in the fluid transfer into or out of the tank, the fluid and heat transfer between nodes, and the heat transfer across the tank boundary. A 
schematic of a tank node with all of the interaction, from Cruickshank [48], is shown in Figure 2-1.

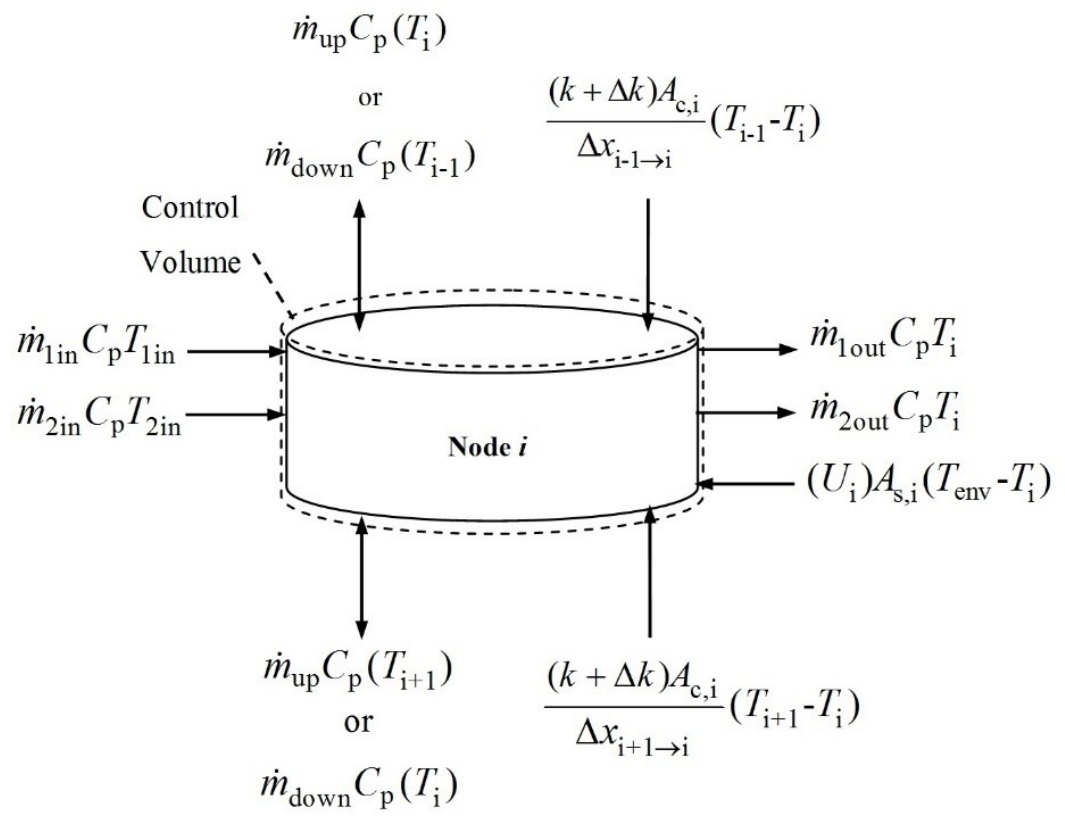

Figure 2-1: Schematic of energy into and out of a single node being modelled in a stratified thermal storage tank

If the tank contains only one node, it is considered to be a fully mixed, and uniform temperature, while increasing the number of nodes increases the resolution and consequently the accuracy of the model, as shown by Cruickshank and Harrison [60]. A first order differential equation defining the energy balance was developed for each node by Newton [61] and have been integrated into a TRNSYS Type, allowing for the modelling of a thermal storage tank within the TRNSYS modelling platform [62]. TRNSYS allows for the simultaneous solving of the $\mathrm{N}$ differential equations though numerical methods, allowing the temperature at each individual node to be determined.

To model the interaction between tank nodes, a set of governing equations is used to model the heat and mass transfer that is occurring within the system. To model the tank a number of assumptions need to be made, including that the fluid is incompressible, steady 
state and constant fluid properties through the simulation. To model the flow between nodes, the conservation of mass, momentum and energy are utilized [48]. Since the control volume is constant for each node, the mass of fluid entering the node must equal the mass exiting, and therefore Equation 2.1 is used.

$$
\frac{\partial u}{\partial x}+\frac{\partial v}{\partial y}+\frac{\partial w}{\partial z}=0
$$

The momentum is also conserved in each of the three directions, and is solved using Equation 2.2, where the partial in the u-direction is repeated in the v- and w-direction.

$$
u \frac{\partial u}{\partial x}+v \frac{\partial u}{\partial y}+w \frac{\partial u}{\partial z}=-\frac{1}{\rho} \frac{\partial u}{\partial z}+v\left(\frac{\partial^{2} u}{\partial x^{2}}+\frac{\partial^{2} u}{\partial y^{2}}+\frac{\partial^{2} u}{\partial z^{2}}\right)
$$

Finally, the conservation of energy for each node is calculated used Equation 2.2.

$$
u \frac{\partial T}{\partial x}+v \frac{\partial T}{\partial y}+w \frac{\partial T}{\partial z}=\frac{k}{\rho c_{p}}\left(\frac{\partial^{2} T}{\partial x^{2}}+\frac{\partial^{2} T}{\partial y^{2}}+\frac{\partial^{2} T}{\partial z^{2}}\right)
$$

These equations are solved numerically by discretizing each node. This allows the equations to be transformed into algebraic equations, with appropriate boundary conditions. This series of equations is then solved iteratively in the using the TRNSYS numerical solver.

This model has been integrated into the standard TRNSYS program as Type 4, and has been extensively used throughout literature to model the performance of, and temperature distribution within thermal storage tanks. That being said, all of the studies using this Type, as well as the corresponding Types 60 and 534, which use the same basic principles to calculate the temperature profile of a cylindrical storage tank, have been conducted on hot thermal storage systems. As such, no previous studies could be found to prove the validity of this method at low temperatures or using a glycol/water solution at 
temperatures below $0^{\circ} \mathrm{C}$. As such, before these models can be used for stratified cold thermal storage systems, the predicted results must be validated.

\subsubsection{Ice Storage}

Stratified storage tanks can provide cost effective thermal storage, however there are many challenges for their use in cold thermal storage applications. As a result of the relatively small temperature differences obtainable for cooling applications, very low thermal storage densities are obtainable. As a result, it is common for cold thermal storages to employ phase change materials, taking advantage of the latent heat of formation. A graph shown in Figure 2-2 from Cruickshank and Baldwin [15] compares the storage density of water, a 50/50 glycol/water solution and ice, with ice storage having a density in excess of 8 times that of a water only storage tank. This allows for a significant amount of cooling

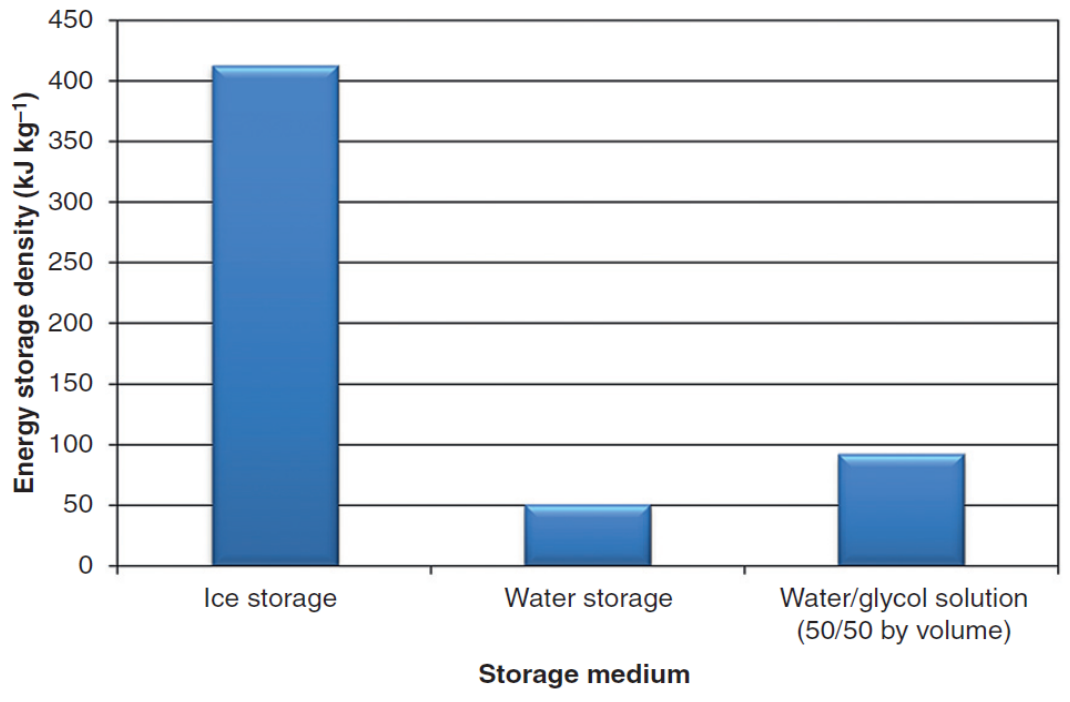

Figure 2-2: Energy storage densities for cold thermal storages

potential to be stored within a relatively small volume.

Ice storage has seen an increase in popularity over recent years, predominantly in large scale, commercial and industrial applications. The principle use for these ice storages is to smooth the electrical consumption required for air conditioning over the 24-hour 
period, as opposed to almost all electrical consumption occurring during peak, daytime periods. To achieve this, three high level control strategies can be employed; 1) a full storage strategy in which all cooling loads are met using stored energy during peak periods, and the chillers run at maximum during off-peak periods, 2) a partial storage strategy with demand limiting, in which chillers supply some of the required cooling and the remainder is met using stored cooling potential and the chillers run at a lower level during peak periods compared to off-peak periods, and 3) a partial storage strategy with load levelling, in which the chiller runs continuously at the same level throughout the day, with the extra cooling stored during overnight periods and used to meet the increase in load during the peak period [63].

Installation of ice based cold thermal storage systems have typically occurred in building that are predominantly occupied during peak, working hours with many examples found in literature of these systems. Of the installed cold thermal storage systems, $80-85 \%$ of these systems use ice storage, with the bulk of the remainder using chilled water and a small percentage using eutectic salts [64]. These ice storage systems can be broken down into four major types: ice harvesting, ice-on-coil (which can be further categorized as internal and external melt), ice slurry and encapsulated ice systems [64, 65]. Each of these systems have their own advantages and disadvantages, however ice-on-coil systems have shown promising results in smaller systems including in a study by Lee and Jones [66] which used an ice-on-coil storage tank with a $150 \mathrm{kWh}$ storage capacity and direct refrigerant coils, and connected to a simulated $10,000 \mathrm{ft}^{2}$ building. Additionally, Sait [67] experimentally evaluated an alternate design, in which the water is misted over chilled pipes for ice development, and using the experimental results, developed a model of the 
system. This system requires a larger volume and does no realize the sensible storage capabilities of the storage medium.

Based on the results found in literature, this study will focus on using a small-scale, ice-on-coil cold thermal storage system. This has been selected to act as it is intended to be a diurnal system, shifting the electrical consumption required to meet the residential cooling load from peak to off-peak periods.

\subsubsection{Modelling Ice Storage Systems}

An important component of this study is performing annual simulations of the proposed system, including the heat pump, hot and cold thermal storage, as well as the residential building. To conduct these simulations, TRNSYS: Transient System Simulation Tool was selected due to its flexibility to model both energy systems and complete buildings, while being easily able to incorporate existing system components from a diverse library, while also being able to develop new, custom components [62]. One such component which will have to be custom built and coded into TRNSYS is the ice storage system. The standard and expanded TRNSYS component libraries do not contain an ice storage tank, while a simple ice storage model is available from the University of Wisconsin, however only one that uses an ice harvesting system and models the ice as a percent of tank that is ice [68]. Although no ice on coil TRNSYS components are publicly available, ice on coil systems have been previously modelled using various platforms.

Ice on coils have historically been modelled using one of two methods. The first is using fundamental heat transfer and thermodynamic properties of the materials, while the second uses a performance map for ice growth rates using experimentally obtained data. A number of different methods can be employed when modelling using first principles. The 
most simplistic is using a lumped capacitance method, first proposed by Jekel et al [69]. This method looks at the complete tank as a lumped system, and only considers tank properties, and the heat transfer into and out of the tank, with the total thermal storage calculated. This model was later validated by Drees and Braun [70], which found this modelling predicts the heat transfer into and out of the storage tank to with an average error of between $3 \%$ and $6 \%$.

Building off of this simple model, a more detailed model was developed by Soltan and Ardehali [71] which set up the freezing and melting of the ice on the submerged pipes as a moving boundary problem, commonly referred to as the Stefan Problems. There does not exist an analytical solution to this problem, which is in the form of a non-linear, parabolic form, and as such, a numerical method must be used to solve the problem. To effectively utilize this method, a number of assumptions must be made. These include that the surface temperature of the pipe is the same temperature as the fluid in the pipe, and that the mass flow rate is large enough that there is a negligible temperature change and the fluid temperature can be assumed constant. Although these assumptions are valid in a number of applications, when modelling smaller thermal storage systems, the flow rates will not be adequately high for this assumption. Additionally, when modelling a complete system, this assumption does not hold, as the premise of the thermal storage is to realize a temperature difference, and to be met using the heat pump.

The major method for modelling ice on coil thermal storage systems is to develop a performance map of analytical solutions for ice growth and heat transfer rate based on the flow rate of the working fluid, temperature of the working fluid, amount of ice already on the coils, temperature of the surrounding fluid, and velocity of flow over the coils. This 
method has been partially utilized by Jekel [72], however on a much larger scale, with flow rates in excess of $80 \mathrm{GPM}(300 \mathrm{~L} / \mathrm{min})$. This method allows for the more detailed modelling of the ice growth and allows for varying ice thicknesses along the coil, while the drawback is a significant amount of experimentally obtained data is required to develop the relationships used within the model.

\subsection{Performance Metrics}

To assess the performance of the individual components and the overall systems, a number of performance metrics had to be selected and developed. These performance metrics were broken down into heat pump performance, thermal storage performance, the interaction of the heat pump and the thermal storage and the annual performance.

\subsubsection{Heat Pump Performance}

The most widely utilized metric to assess the performance of a heat pump is to use the coefficient of performance, which is the ratio of electrical input to the heating or cooling provided and is calculated using Equations 2.4 and 2.5 [13].

\subsubsection{Thermal Storage Performance}

$$
\begin{gathered}
C O P_{\text {Heating }}=\frac{Q_{\text {Load }}}{e_{\text {Heat_Pump }}} \\
C O P_{\text {Cooling }}=\frac{Q_{\text {cold }}}{e_{\text {chiller }}}
\end{gathered}
$$

To assess the performance of the thermal storage system, the total storage at any given point is calculated in two different methods. The first is an energy balance across the boundary of the tank, using Equation 2.6.

$$
E_{\text {Stored }}=\sum_{i=1}^{n} \dot{m} c_{\mathrm{p}} \Delta T_{\text {source }} t_{\mathrm{i}}-\dot{m} c_{\mathrm{p}} \Delta T_{\text {load }} t_{\mathrm{i}}-k \Delta T_{\text {wall }} A t_{\mathrm{i}}
$$

Using the basics of this equation, a second parameter can be determined which is the effectiveness of the thermal storage, which is the ratio of energy supplied to the storage 
system to that delivered to the load, and is calculated using Equation 2.7.

$$
\varepsilon=\sum_{i=1}^{n} \frac{\dot{m} c_{\mathrm{p}} \Delta T_{\text {load }} t_{\mathrm{i}}}{\dot{m} c_{\mathrm{p}} \Delta T_{\text {source }} t_{\mathrm{i}}}
$$

This equation takes into account the loss from within and through the walls of the storage tank, and provides and indicator as to the performance of the storage system. The second method for calculating the energy stored when using a sensible thermal storage system is to use the thermodynamic properties of the storage medium, the physical properties of the tank and the temperature distribution within the tank. This is done using Equation 2.8.

\subsubsection{Annual System Performance}

$$
E_{\text {Stored }}=\sum_{i=1}^{N} \rho V c_{\mathrm{p}}\left(T_{\mathrm{i}}-T_{\text {ref }}\right)
$$

Once the performance of the individual components has been calculated and used to optimize the system performance, performance metrics have to be developed to assess the annual performance of the system. The first was the annual coefficient of performance of the system, which factored in the heating and cooling loads of the house, and the electrical consumption of the heat pump plus all associated components (pumps and fans) as shown in Equation 2.9.

$$
C O P_{\text {annual }}=\frac{E_{\text {heating }}+E_{\text {cooling }}}{E_{\text {electrical }}}
$$

In conjunction to calculating the annual system performance, a number of metrics are required to assess the ability for the proposed systems to shift electrical consumption from 
peak to off-peak periods. The main parameter is the percent consumption during heating and cooling season, as defined in Equation 2.10.

$$
\% \text { Peak Consumption }=\frac{E_{\text {Elec,Peak }}}{E_{\text {Elec, }, \text { Total }}}
$$

The lower the \% consumption, the more effective the strategy is at effective demand side management.

\subsection{Gaps in Current Literature}

Based on the findings of this literature review, a number of gaps have been identified. A larger focus has been placed on commercial and industrial demand side management, with less work being done on residential applications. This focus has predominantly been due to commercial application's economies of scale where measures can have a much larger impact on energy use and utility costs. This is because of the larger, predictable loads, more space for system integration and the fact that commercial buildings have a larger swing in peak to off peak costs, as well as billings based on their monthly peak consumption. The work that has been conducted within the residential sector has focused on large scale programs that provide incentives and programs consumers can enroll or participate in, or that change the electrical utility structure, like in the implementation of time-of-use billing. Although some systems that use electrical resistance heaters with thermal storage are commercially available, no systems using heat pumps and thermal storage for demand side management on a residential scale are currently available, or coming on to the market. To determine whether this type of system could be economically beneficial, detailed annual modelling is required, however very little has been done on the development and validation of computer models for small-scale cold thermal storage systems. This research will focus on developing a system that utilizes a heat pump with 
both hot and cold thermal storage systems to offset peak and mid-peak electrical consumption. Through the course of this work, detailed computer models for different types of small-scale cold thermal storage systems will be extensively examined, with a focus on Ontario applications, but examining their applicability across North America. 


\section{Chapter 3: Experimental Set-Up}

A total of three experimental set-ups were designed and constructed with the focus on obtaining experimental data to develop and validate computer models of the individual components. These are 1) an experimental set-up to evaluate the performance of heat pump connected to thermal storage, 2) an experimental set-up to evaluate the performance of a medium temperature chiller, and 3) an experimental set-up to measure the performance of an ice based thermal storage system. The first set-up consisted of a standard, liquid to liquid heat pump designed for use as a ground source heat pump, installed between a hot and cold sensible thermal storage system. In this system, energy flowed from the cold thermal storage system (reducing its temperature), through the heat pump, where it was upgraded to a higher temperature, and then transferred to the hot thermal storage (increasing its temperature). This set-up first used water as the cold storage medium, and then was replaced with a 50/50 by volume, water/glycol solution, allowing lower temperatures to be obtained. The second set-up was a retrofit of the first experimental set-up, where the standard ground source heat pump was replaced with a medium temperature chiller, which had a higher cooling capacity and was able to reach much lower temperatures then the standard heat pump. This set-up was used to experimentally evaluate the performance of the medium temperature chiller under varying flows and temperatures. The final experimental set-up developed as part of this project was a bench-top ice storage model. This consisted of a single instrumented pipe, submerged within a water tank, where glycol chilled below $0^{\circ} \mathrm{C}$ is circulated through the pipe, and the heat transfer into/out of the water tank, the temperature of the water, and the growth of ice on the pipe were measured. Detailed descriptions of each experimental set-up, the instrumentation used and the 
measurements to be taken are provided below.

\subsection{Liquid to Liquid Heat Pump Coupled with Sensible Thermal Storage}

The first experimental test set-up that was constructed, instrumented, and commissioned to experimentally evaluate all aspects of a typical liquid to liquid heat pump connected to both a hot and cold sensible thermal storage. This includes measuring the steady state performance of the heat pump, the temperature profile through both the hot and cold thermal storage tanks, the charge and discharge profiles and the interaction between the thermal storage systems and the heat pump. This system was integrated into the existing infrastructure within the Solar Energy Systems Laboratory at Carleton University, providing a continuous supply of both hot water for heating and chilled water for heat rejection. This allowed for the controlled addition and subtraction of energy to the different systems and was used to mimic the space heating and cooling loads of an actual house. Finally, the system was also connected to an existing hot water draw system, allowing for the future inclusion of the proposed systems supplying the domestic hot water. A photo of the complete system is shown in Figure 3-1, while a complete schematic of the experimental system as constructed is provided in Figure 3-2.

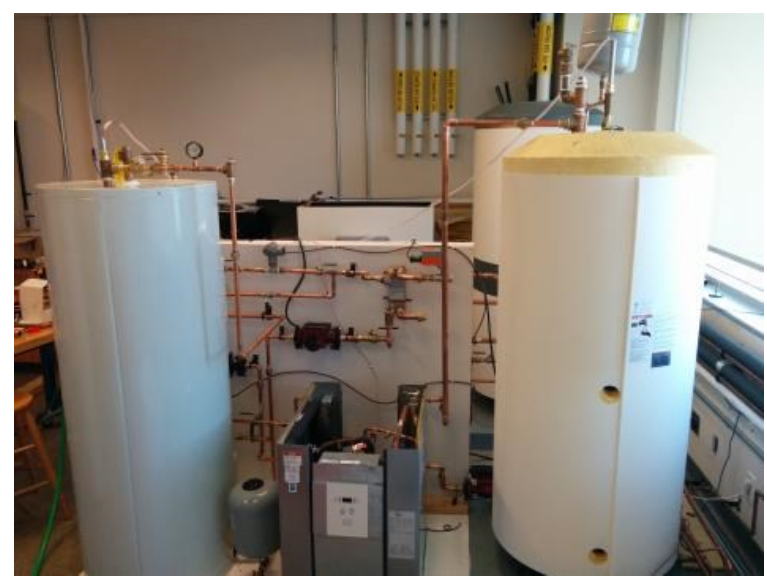

Figure 3-1: Photo of heat pump (center) with cold (left) and hot (right) thermal storage systems 


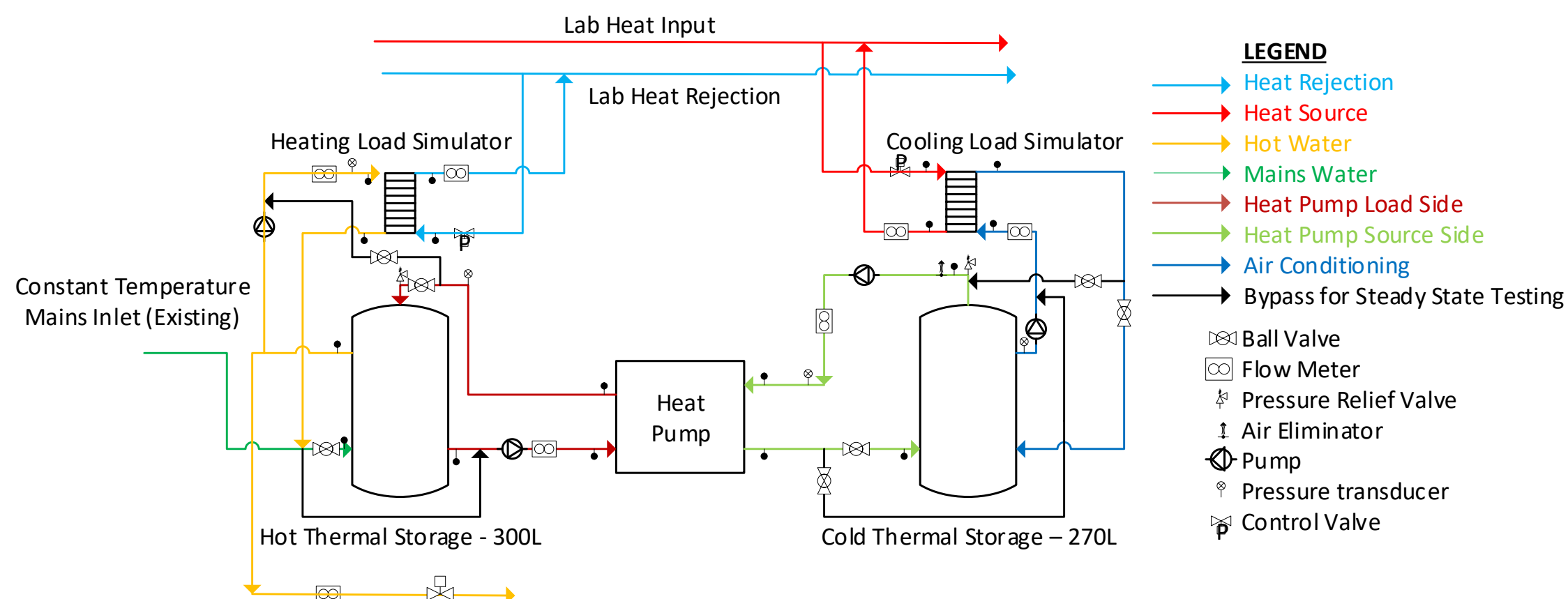

Hot Water Draw Simulator

Figure 3-2: Schematic of completed experimental test set-up 
To measure the performance of the system, instrumentation was installed throughout the system. This included 35 T-Type thermocouples, located at the inlets and outlets of the heat pump, the thermal storage tanks, and the heat exchangers, as well as in the storage tanks, using a temperature probe, as described in Baldwin [73]. To measure the flow rate within each of the four loops, Blancett 1100 impeller flow meters were installed. This instrumentation allowed the heat transfer rates to be calculated through the heat pump, as well as the amount of energy stored within the system. Through these measurements, the impact of flow rates on the thermal storage systems and the overall system performance were determined, which included the impact of the stratification within the storage tanks. The final main piece of instrumentation installed was a WattNode power meter, which was used to measure the electrical consumption of the heat pump.

The experimental test set-up was first utilized to develop a steady state performance map of the heat pump is included in Appendix B, while the experimental uncertainty is discussed and presented in Appendix C. The performance map uses the source and load side entering fluid temperatures and the flow rates as the independent variables, and the power consumption of the compressor and the heat transfer rate from the source fluid and to the load fluid as the measured dependent variables. Tests were first performed using water on the source (cold) side of the heat pump with source and load flow rates of $3 \mathrm{~L} / \mathrm{min}$, $6 \mathrm{~L} / \mathrm{min}$, and $9 \mathrm{~L} / \mathrm{min}$, and entering source side temperatures of $10^{\circ} \mathrm{C}, 15^{\circ} \mathrm{C}, 20^{\circ} \mathrm{C}$, and $25^{\circ} \mathrm{C}$, while the load side entering temperatures were evaluated at $20^{\circ} \mathrm{C}, 25^{\circ} \mathrm{C}, 30^{\circ} \mathrm{C}, 35^{\circ} \mathrm{C}$, and $40^{\circ} \mathrm{C}$. In total, 180 tests were conducted. Before conducting the steady state tests, a number of preliminary tests were conducted to determine the length of time required to reach steady state. Through these tests, it was determined that a 15 minutes stabilization 
period was required to achieve steady state flow rates and outlet temperatures. Each test consisted of setting the inlet temperature and flow rates, and once the inlet conditions on both the load and source side reached steady state through the 15-minute stabilization period, data was collected for 10 minutes at 30 second intervals. The inlet and outlet source and load temperatures and the flow rates were then averaged for each test, and the heat transfer rates into the load fluid stream and out of the source fluid stream were calculated. Additionally, the average power consumption of the compressor was measured and averaged over the 10-minute period. The results of each test were then tabulated, and a complete performance map was created.

As water was being used as the test fluid, if the outlet source temperature dropped below $3^{\circ} \mathrm{C}$, the heat pump turned off to prevent any potential freezing and consequential damage. Additionally, the refrigerant temperature entering and leaving the compressor was monitored, and if the exiting refrigerant temperature was above $56^{\circ} \mathrm{C}$, the test was stopped due to high pressure in the refrigerant line (this corresponds to approximately $3.55 \mathrm{MPa}$ (515 psi) for the refrigerant being used, R-410a). As a result, tests with a source flow rate of $3 \mathrm{~L} / \mathrm{min}$ and entering source temperatures of $10^{\circ} \mathrm{C}$ and $15^{\circ} \mathrm{C}$ were not completed, and a number of tests at high load entering temperature failed due to the high refrigerant pressure limit. The performance of the heat pump at each inlet temperature and flow rate tested are tabulated in Appendix B, while the uncertainty on these values is given in Appendix C.

Once the storage medium in the cold storage was switched from water to a 50/50 by volume water/glycol solution to allow much lower storage temperatures, the process was repeated with the water/glycol solution on the source side. Due to the decreased heat transfer possible with the water/glycol solution, the heat pump had a reduced high 
temperature range, and as a result, no tests were conducted with a load side temperature of $40^{\circ} \mathrm{C}$ as the heat pump will not function. As the water/glycol solution has a freezing point below $-35^{\circ} \mathrm{C}$, lower source side temperatures can be achieved and as a result, an additional source side temperature of $5^{\circ} \mathrm{C}$ was added to the test as much lower temperatures are possible when using a glycol solution. The same test procedure was utilized as the tests conducted with water as the source side fluid, with the results of these tests tabulated in Appendix B, with the uncertainties in Appendix C. These values were then used to develop a performance map in a format that could be read into TRNSYS to model the performance of the heat pump.

In addition to determining the performance of the heat pump, this set-up was used to assess the performance and temperature profile within the thermal storage. This was done using the temperature probe installed within the cold tank, in which 13 T-Type thermocouples were installed within a stainless steel pipe through the center of the tank, as shown schematically in Figure 3-2 The first thermocouple was installed $5 \mathrm{~cm}$ from the bottom of the tank, and each subsequent thermocouple was incremented $10 \mathrm{~cm}$ higher, with the last thermocouple installed $5 \mathrm{~cm}$ from the top of the tank. An image of this thermocouple probe is shown in Figure 3-3. Using these thermocouples, a detailed temperature profile of the tank can be measured over the course of an experiment and using this data the stratification within the tank can be determined. An example of the temperature profiles obtained is shown in Figure 3-4, and these profiles were used to validate computer models of the storage tanks. In this case, each line represents the temperature of a single thermocouple over the course of a test. In a cold thermal storage application, the tank is charged from the bottom of the tank to the top of the tank, so the 
first node to change temperature is the bottom, and then in order until the top node begins to charge, before the bottom node begins to further charge. A second thermocouple probe was installed in the hot tank to measure the temperature profile in the tank. A total of 10 type-T thermocouples were installed in the same method as those installed in the cold tank, spaced at $15 \mathrm{~cm}$ increments.

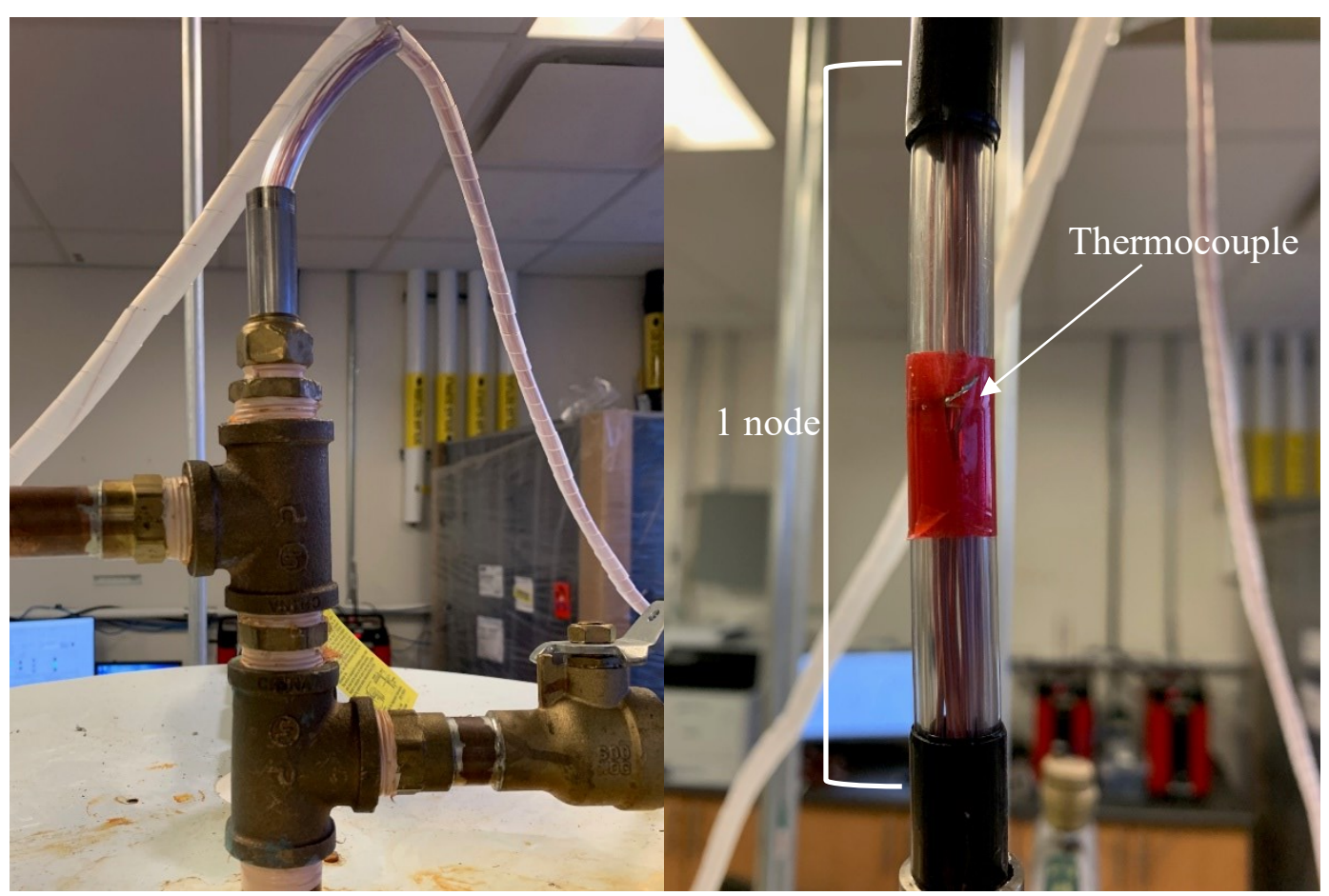

Figure 3-3: Thermocouple probe as it enters the tank (Left) and the thermocouple installed within the tank (Right) 


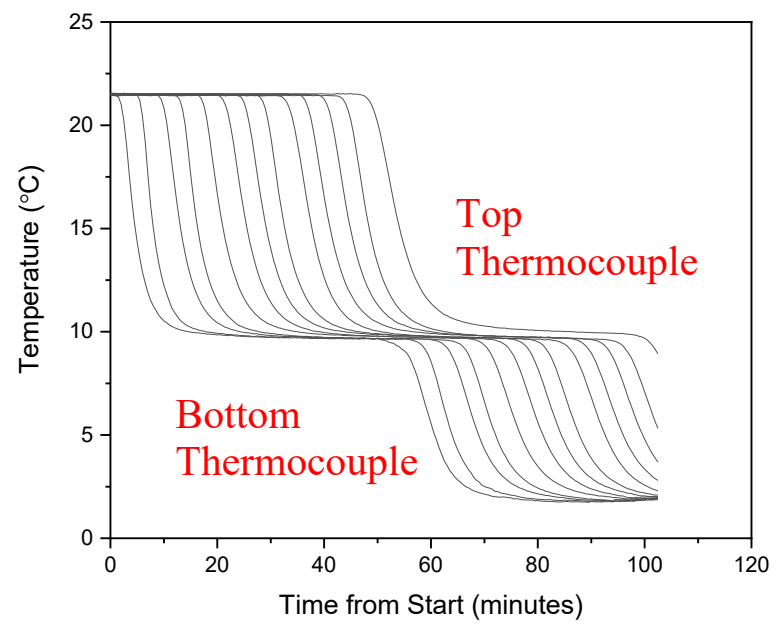

Figure 3-4: Example of a temperature profile at low flow rates in the cold thermal storage

In addition to determining the temperature profile of the tank, the temperature probe in the cold tank was also used to determine the amount of energy or cooling potential stored in the tank. Each thermocouple represents $1 / 13^{\text {th }}$ of the volume of the tank, and through tracking the change in temperature of a given point from the start of the test to any point during the test, the change in energy for each node was determined, and by summing all 13 nodes, the total change in energy in the thermal storage was calculated. This was used to determine the total energy stored within a tank, and to calculate the storage efficiency of the thermal storage system. During all tests, the temperature profile and total energy storage were recorded and calculated respectively.

\subsection{Medium Temperature Chiller Evaluation}

After testing concluded on the standard liquid to liquid heat pump, it was determined that the standard liquid to liquid heat pump could not reach low enough temperatures to obtain high cooling potential storage densities (be it low temperature sensible storage or ice storage). To examine the potential of using these thermal storage strategies, a heat pump or chiller that operated with average evaporator temperatures in the range of $-5^{\circ} \mathrm{C}$ to $-15^{\circ} \mathrm{C}$ could be used. This would allow for the either lower temperature 
sensible storage, which would increase the amount of cooling potential stored for the same volume of fluid. A lower temperature liquid to liquid chiller typically used for walk-in fridges and freezers was sourced and installed within the experimental set-up. New pumps were installed to meet the increased flow and head requirements of the medium temperature chiller, but the remainder of the experimental system was maintained, including the hot and cold thermal storage and all instrumentation. A series of images of the chiller and new piping installed to accommodate the medium temperature chiller are shown in Figure 3-5.

This set-up was used in a similar method as the set-up testing the ground source heat pump, but as a result of both the higher capacity of the medium temperature chiller and the lower temperatures obtainable, a larger test range was utilized in the development of the performance map of the system. The chiller was tested at cold side flow rates of 5, 10 and $15 \mathrm{~L} / \mathrm{min}$ and temperatures of $-5^{\circ} \mathrm{C}$ to $25^{\circ} \mathrm{C}$ in $5^{\circ} \mathrm{C}$ increments, while the hot side was tested at 10,15 and $20 \mathrm{~L} / \mathrm{min}$ and entering temperatures of $15^{\circ} \mathrm{C}$ to $35^{\circ} \mathrm{C}$ in $5^{\circ} \mathrm{C}$ increments. This resulted in a total of 315 test points, although a number of tests were not able to be completed. These failed tests included those at low flow rates, with a cold side temperature of $-5^{\circ} \mathrm{C}$, and $/$ or hot side temperatures of $35^{\circ} \mathrm{C}$. The low temperature tests failed due to localized freezing of the heating water in the heat exchanger which provided heat input to the system. This stopped the flow through the systems, leading to a low-pressure cut-off. Additionally, insufficient pump power was installed in the system to meet the flow set-points when the glycol/water solution was at very low temperatures. This was caused by the significant increase in the viscosity of the heat transfer fluid and consequently the pumping power required to move the fluid, which led to tests that did not maintain the required flow rate. The tests using a hot side inlet temperature of $35^{\circ} \mathrm{C}$ and low flow rates 

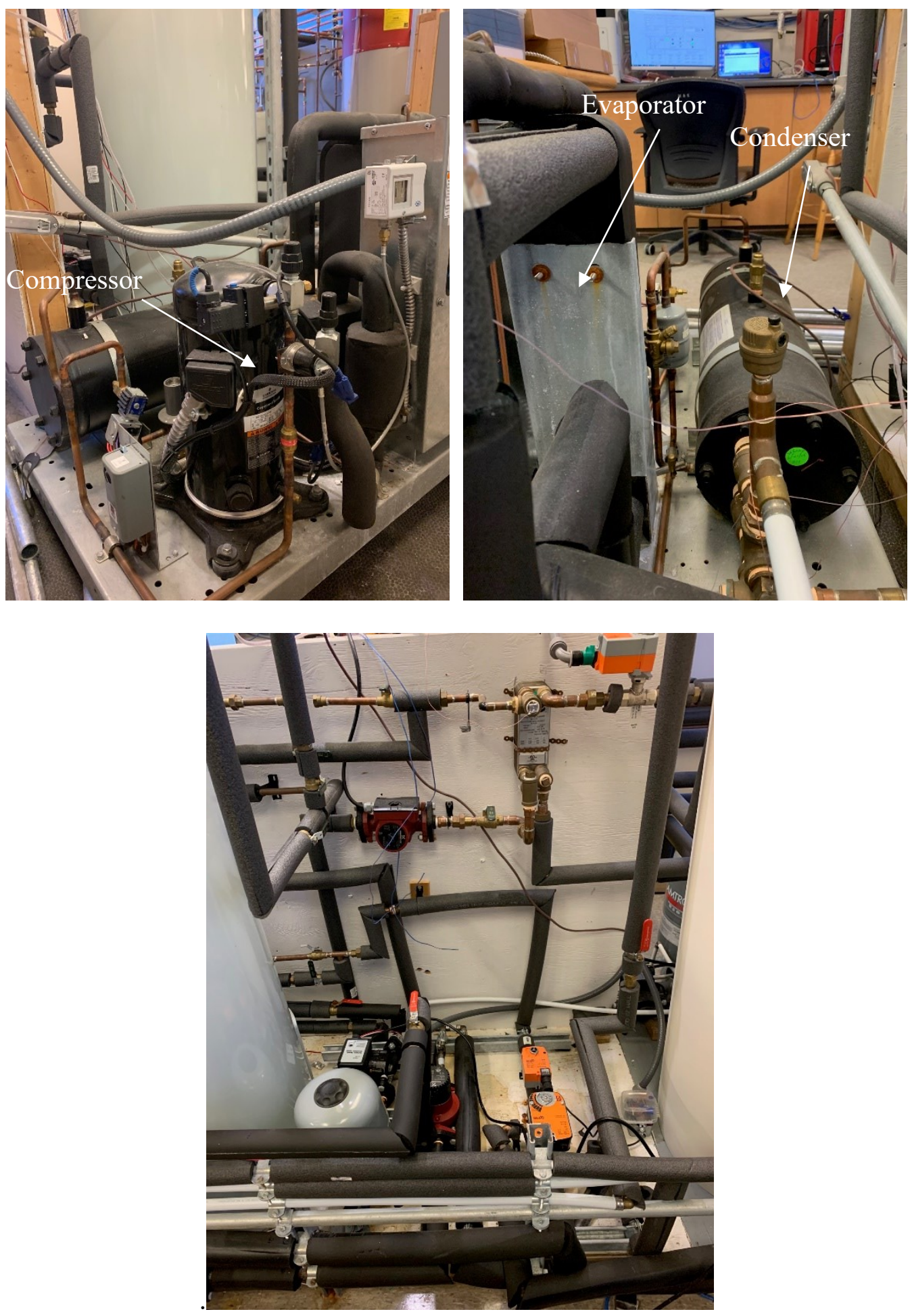

Figure 3-5: Images of the medium temperature chiller set-up, including the components of the chiller (top) and the required infrastructure to operate and test the chiller (bottom) 
also result in failed tests, as a result of insufficient heat rejection available based on the temperature and flow conditions, which caused a high-pressure safety cut-off to be triggered, turning off the chiller. As such, these values were extrapolated from the remainder of data to ensure a complete performance map could be developed and implemented within TRNSYS. The performance data for the chiller is tabulated in Appendix B, while the uncertainty on these measurements is provided in Appendix C.

This medium temperature chiller was also used to provide the chilled glycol solution used within the ice storage set-up (discussed in Section 3.3), using a separate thermal storage tank, in which a 50/50 water/propylene glycol solution was stored at a set point between $-5^{\circ} \mathrm{C}$ to $-10^{\circ} \mathrm{C}$ and pumped through the ice storage system. This meant that both the chiller and the ice storage test apparatuses could not operate at the same time.

\subsection{Ice Storage Small Scale Test Set-Up}

Through experimental testing and modelling of the proposed systems (discussed in Chapter 4 and 5), it was determined that the sensible cold storage systems did not have the required storage density to be feasibly implemented into a residential building. To increase the storage density of the cold storage system, an ice storage system was proposed. To determine whether this type of system is compatible with the chiller and heat pump systems tested, and technically feasible, a model of the storage system was required. A small scale set-up was designed, built and commissioned to measure the heat transfer through the pipe into the thermal storage, and the measure the rate of ice growth on the submerged pipe. The experimental data would be used for validating the developed model of the ice storage system.

Although in an actual application, an ice thermal storage would have a volume in 
the order of magnitude of $300 \mathrm{~L}$ to $600 \mathrm{~L}$ with between 50 and 200 submerged pipe, it was not feasible to construct a full-scale system and instrument it to determine performance. Additionally, it was important to test and validate the systems using a simple set-up, limiting the variables at play, then once validated and the concept proven, a full-scale setup could be explored. As such, a small-scale tank was built with a single, submerged pipe. The system was designed to measure the heat transfer through this pipe, as well as the amount of ice that builds up on the pipe through the test. This data was then used to validate the computer models of the ice storage system. Once the system was validated for a single pipe, the model was then extrapolated to represent a larger system, and used to determine its feasibility as a cold thermal storage system for residential demand side management.

The test set-up consisted of rectangular tank, constructed of acrylic sheets, with a cross section of $30 \mathrm{~cm} \times 30 \mathrm{~cm}$, and a length of $1.3 \mathrm{~m}$. On the inside surface of the acrylic t, $10 \mathrm{~cm}$ of extruded polystyrene sheets were then glued on the inside of the acrylic sheets to both provide insulation to the tank and reduce the volume of the tank to what would be more indicative of an actual submerged pipe, ice storage system. This left a volume of approximately $10 \mathrm{~cm} \times 10 \mathrm{~cm} \times 1.3 \mathrm{~m}$. A single copper pipe, with an outer diameter of 9.5 $\mathrm{mm}$ and a wall thickness of $0.75 \mathrm{~mm}$ was installed through the center of the tank volume. This pipe is connected to the system using compression fittings, which would allow for different pipe diameters and configurations, including pipe materials and possibly pipe geometry to be tested in the future. The pipe is then connected to a supply of chilled glycol, and connected to a variable speed pump that allows varying flows to be tested through the test pipe. Originally, a heat exchanger was installed between the reservoir of chilled glycol and the test pipe to allow for additional control of the temperature and flow through the 
pipe; however, after initial testing of the system, the heat loss through the heat exchanger was too great, and the lowest temperature that could be obtained was between $-2^{\circ} \mathrm{C}$ and $3^{\circ} \mathrm{C}$, which was not low enough to obtain adequate heat transfer to produce ice on the test pipe. As such, the heat exchanger was removed and the test pipe was connected directly to the chilled glycol reservoir, which is capable of storing glycol between $-5^{\circ} \mathrm{C}$ to $-11^{\circ} \mathrm{C}$. This allows a continuous supply of constant temperature chilled glycol through the set-up, providing adequate cooling to chill the tank and generate ice on the test pipe which could be measured.

\subsubsection{Instrumentation within the Ice Storage Test Set-Up}

The tank and test pipe were extensively instrumented to measure the heat transfer occurring within the system, as well as the total amount of energy stored within the system. To determine the amount of heat transferred from the tank into the chilled solution, a flow meter was installed to determine the mass flow rate of the fluid. Three thermocouples were installed at each the inlet and the exit of the test pipe, submerged in the flowing fluid. During installation, one thermocouple at the inlet was damaged, resulting in two functioning thermocouples at the inlet, and three at the outlet. The thermocouples were calibrated to an overall uncertainty of $\pm 0.25^{\circ} \mathrm{C}$, and the average of the thermocouple temperature readings was taken.

When taking the temperature difference between the inlet and outlet of the test pipe, this uncertainty was compounded to $\pm 0.35^{\circ} \mathrm{C}$, which when compared to the typical of change in temperature across the pipe, resulted in a significant experimental uncertainty approaching $20 \%$. A preferred method to measure this change in temperature across the length of the pipe is to use a multi-junction thermopile. This is a series of thermocouples, 
which uses one side as the refence junction, and measures the change in temperature between the two junctions. As it is a series of thermocouples, this amplifies the signal, as well as removes the requirement for cold junction compensation. This reduces the uncertainty on the change in temperature across the pipe from $\pm 0.35^{\circ} \mathrm{C}$ to between $\pm 0.05^{\circ} \mathrm{C}$ and $\pm 0.1^{\circ} \mathrm{C}$ depending on the thermopile configuration and accuracy of the calibration. Unfortunately, at the time the test apparatus was constructed, the lab did not possess the equipment required to properly calibrate the thermopiles at the expected temperatures. A second alternative solution that would have reduced the total uncertainty would have been to use a secondary reference RTD at both the inlet and outlet of the system. This was decided against for two reasons. RTDs are extremely expensive and were not possible within the budget for this set-up, and they typically have slower response times and therefore are much better suited for systems that operate only at steady state. As a result, it was decided that the three embedded thermocouples were the best option available.

In addition to the thermocouples installed to measure the total heat transfer across the length of the pipe, thermocouples were also installed to measure the surface temperature along the length of the submerged pipe, as well as the temperature of the water in the storage tank. A total of seven thermocouples were installed using a high conductivity thermal epoxy, with the intention that the thermocouples would measure the surface temperature of the pipe. Because the pipe was so thin, and the epoxy layer aproximatley doubled the thickness, the thermocouple ended up reading a temperature slightly higher than that of the actual surface temperature, however, the series of thermocouples down the length of the pipe still showed the difference in temperature along the length of the pipe. An image of the epoxied thermocouple is shown in Figure 3-6. 


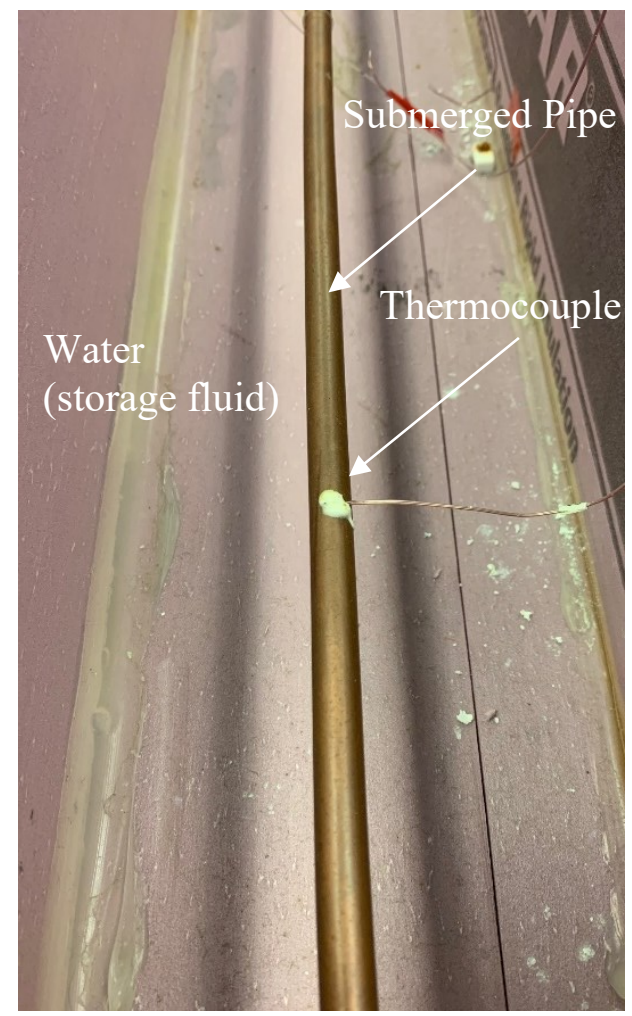

Figure 3-6: Thermocouple epoxied to the outer surface of the submerged pipe

The second set of thermocouples were used to measure the temperature of the water within the tank. To get an accurate distribution across length and depth of the tank, three sets of three thermocouples were installed within the tank. The sensors were installed at radial distance from the surface of the submerged pipe, at distances of 2,4 and $6 \mathrm{~cm}$. The thermocouples were attached to fishing line to minimize their impact on the temperature distribution. In the first and third set of thermocouples, located $1 / 4$ of the distance from each end of the tank, the sensors at 2 and $6 \mathrm{~cm}$ were installed above the pipe, and the sensor at $4 \mathrm{~cm}$ was installed below, while the reverse was true in the middle set of thermocouples. The submerged thermocouples are shown in Figure 3-7. 


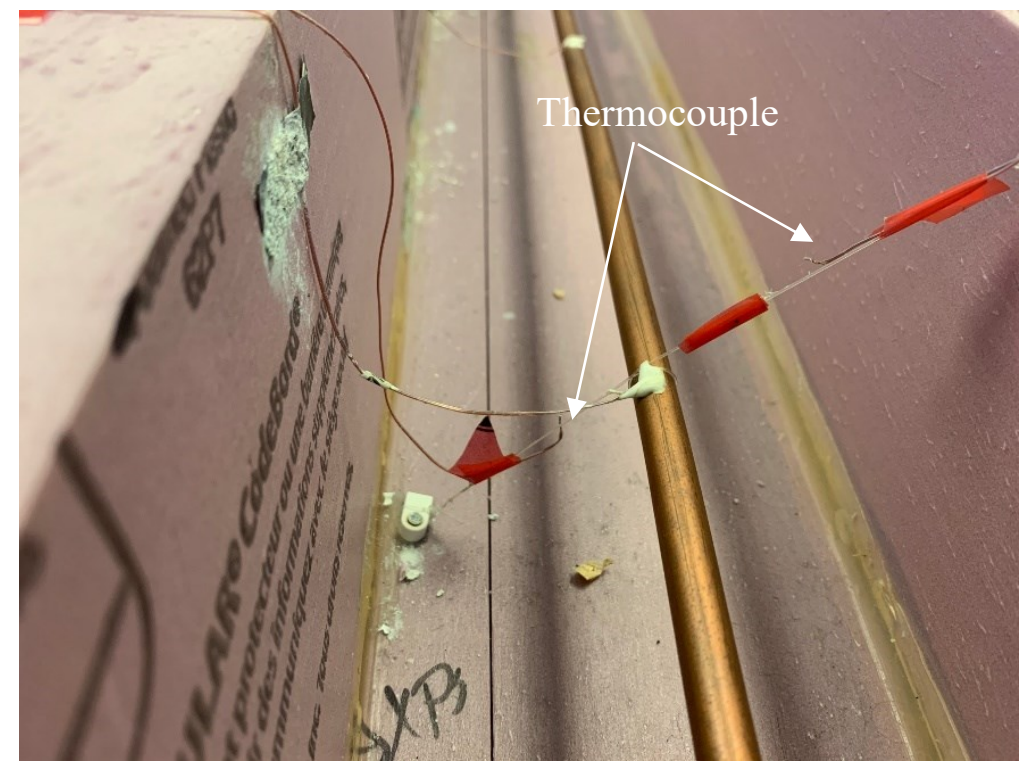

Figure 3-7: Thermocouples connected to fishing line to allow the fluid temperature around the pipe to be measured.

In addition to the temperature measurements, instrumentation was added to measure the flow rate of fluid through the submerged pipe, and the ice buildup on the submerged pipe. The flow was straight-forward, with an impeller flow meter capable of measuring flows down to $1 \mathrm{~L} / \mathrm{min}$, which read into the data acquisition using pulse outputs. This flow meter had an accuracy of $\pm 1 \%$ of the reading plus the flow represented by 1 pulse output. The measurement of the ice build-up was much more problematic and a number of different solutions were attempted. The first strategy was to leave the top off of the tank and use time lapse photos of the tank to record ice growth. This quickly failed as the removal of insulation on the top of the tank created excess heat gain from the environment into the storage. Additionally, any time people walked by the set-up, the water rippled distorting the images, which made it almost impossible to analyze the images. Finally, for accurate measurements, a camera would have been required at each node, requiring significant infrastructure and cameras. 
The second proposed method for measuring the build-up of ice on the submerged pipe was to use infrared (IR) distance sensors. The sensors came calibrated and with defined curves for distances measured through air. As the sensors were not waterproof, a test was conducted to determine whether the IR signal could pass through the water and measure the distance to the surface of the pipe. In initial testing, it was determined that the sensors did transmit through the surface of the water and provide a reading based on the distance from the surface of the water to the surface of the pipe. As such, it was proposed that the sensors could be calibrated to measure the distance to the surface of the pipe. Although initial testing looked promising, through a series of calibration tests, it was determined that the sensors did not have the required resolution to accurately measure the ice growth rates. Additionally, small changes in conditions, such as the air and water temperatures, would impact the relationship between the output of the sensors and the measured distances. As such, new strategies were required that would meet the accuracy and measurement requirements of measuring ice growth.

The third and ultimately chosen solution was to use a linear variable differential transducer (LVDT), which is an accurate method for measuring vertical distance, with accuracies of $\pm 1 \mathrm{~mm}$. This provided the accuracies and repeatability required for experimental evaluations, however, this did not provide an automated solution, as the sensors could not be installed and left, because during tests, the ice would have simply grown around the sensor. As such, the sensors would be removed, and at predetermined time steps, the operator would probe at the center of each of the 6 segments of pipe that make up the fully submerged pipe. The LVDT is inserted into the tank through 6 openings in the top insulation, to a retaining collar to ensure the distances are measured from the 
same point at each time step. The difference between the first measured distance as the test starts, and the distance measured at any given time will give the amount of ice that has formed in the pipe. This measurement is then used to determine the total mass of ice that has formed. An overall photo of the experimental set-up with the lid off (left) and lid on with the probe holes (right), is shown in Figure 3-8.
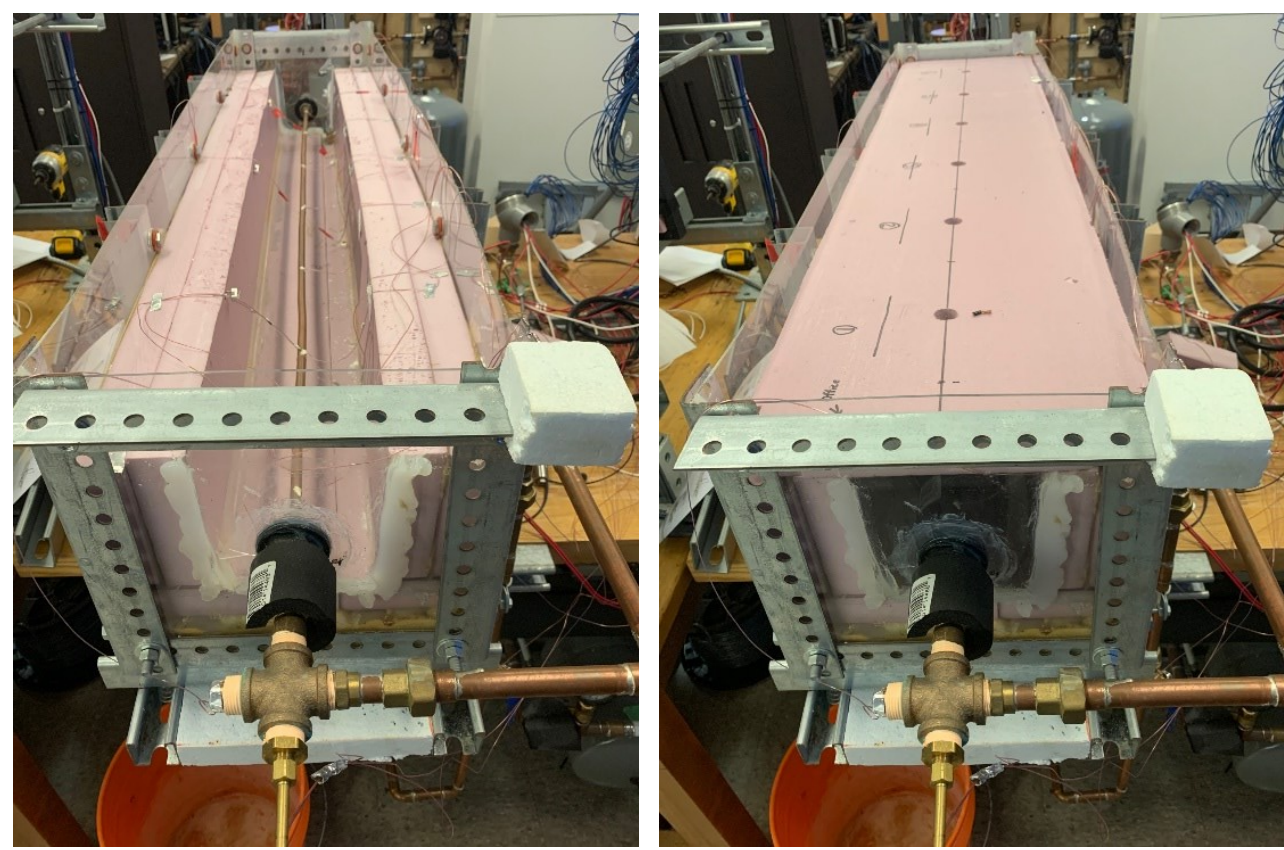

Figure 3-8: Images of the completed small-scale ice storage set-up

\subsection{Conclusion on Experimental Set-Ups}

In this chapter, a description of, and the design process for each of the three main experimental set-ups has been provided. These include a set-up to characterize the steady state performance of a standard liquid to liquid heat pump and a medium temperature chiller. Through the changing of the configurations, the same set-up was used to measure and characterize the interaction between the heat pump and medium temperature chiller and the hot and cold sensible storage tanks. These systems were completely instrumented to measure and monitor the flow of energy through the systems and track the quantity of 
heating and cooling potential being stored. The final experimental set-up measured the performance of a small-scale ice storage system, which was used to validate the developed computer models. This system was used to measure the cooling potential being stored within the system, the heat transfer rates, and the quantity of ice forming on the surface of the pipe. These experimental systems allow the most important components of the proposed demand side management system to be experimentally tested and validated, for use within the annual simulations performed. 


\section{Chapter 4: Component Modelling and Validation}

With the overall research objective of determining if the integration of a heat pump with thermal storage systems can be effectively used for demand side management, extensive modelling of the proposed systems was required. To build a model of the complete system, it was first required to develop and validate models of each of the main components of the overall systems. This includes the different heat pumps being tested throughout the study, the thermal storages being examined, as well as the interaction and performance when the systems are integrated together. These modelled systems would then be integrated into a house model to determine the annual performance and its potential for demand side management. The main purpose of the three experimental set-ups described in the preceding section was to validate the individual components. The experimentally obtained data was used to both develop the performance data and maps the models read in, as well as to obtain data to validate the output parameters of the model. This chapter outlines the models used for the individual components, including the development of a new component for the ice storage, and the validation of these models against experimental data.

Within this study, it was decided that all modelling would be conducted using the Transient System Simulation Tool (TRNSYS). TRNSYS is a program that is designed to model energy systems and uses a graphical user interface. Each component of the actual system is modelled using a mathematical code that represents the physical performance of a component as part of the actual system. Each of these individual pieces of code is called a Type in TRNSYS and contains the mathematical code, as well as a set of static parameters, inputs and outputs. Each Type reads in inputs from other components, runs the 
mathematical code, and produces outputs that can either be read into other components, recorded in an output file for future analysis, or both. The simulation engine uses an iterative solver across all of the Types within the simulation, and once the convergence tolerance is met, will move on to the next timestep in the simulation, using the results from the previous time step as the simulation starting point.

\subsection{Heat Pump Modelling}

The first component which was modelled and experimentally validated was the liquid to liquid heat pump. Through the course of this study, two different heat pumps were tested and modelled within the systems. The first was a standard liquid to liquid ground source heat pump, designed for residential heating applications. This was a small unit with a peak heating power of approximately $6 \mathrm{~kW}$, using a $1.5 \mathrm{~kW}$ compressor. The second was a medium temperature chiller, which is designed to operate with evaporator temperatures between $0^{\circ} \mathrm{C}$ and $-10^{\circ} \mathrm{C}$. Although this is a "chiller" it operates the same as the traditional heat pump, but it does not have the ability to reverse the flow of refrigerant, meaning that the cold and hot side cannot switch. This met the needs of this system, as it was designed in operate in a single direction, from one thermal storage systems to the second. Additionally, in this application, the heat pump was only operated in one direction, and consequently the reversing function was never utilized.

\subsubsection{Modelling the Standard Liquid to Liquid Heat Pump}

To model the standard liquid to liquid heat pump, TRNSYS Type 927 from the Thermal Energy System Specialists (TESS) libraries was selected. This model uses a steady state performance map, inputted as a text file. The performance map of the system uses four independent variables, being the flow on the source (cold) side and load (hot) 
side of the heat pump, as well as the temperature of the fluid entering the evaporator and condenser. The measured heat transfer rates and compressor power were compiled and are shown in Appendix B. This data was then correlated into the correct format, and a text file was created which the Type could read in, as shown in Appendix D. A separate file was used for each of the heat and cooling supplied by the unit. This was first conducted using water on the source (cold) side, and then repeated with a solution of 50/50 glycol/water.

Once this file was developed, a simple TRNSYS model was created that allowed the performance of the heat pump to be calculated, and the outputs were compared directly to the experimental outputs. The comparison of these two results were then be used to validate the model of the traditional liquid to liquid heat pump. To complete the validation of the individual component models, the experimental and modelling results at each time step were examined. This included examining the experimental uncertainty on the measured value and comparing the range of potential actual values from the experiment to the modelled value. The modelled value was first checked to determine if it was in the experimental range, and if the value was outside the experimental value, further investigation was performed. This was to determine if there was a reason the modelled value is outside the experimental range (physical conditions, assumptions, etc.). If the discrepancy could not be accounted for via experimental or modelling procedures and the modelled values consistently fell outside the experimental values, changes and further model development and utilization (e.g. change of parameters) to improve the accuracy and validate the models.

A simple experiment was conducted, where the flow rates through the heat pump were kept constant, and the inlet temperature conditions were varied. Three different flow 
rates were used for each of the source fluids to ensure the validity across all flows and temperatures. The inlet and outlet temperatures along with the flow from the experimental test runs were recorded and a text file was then developed that contained the flow rates and the inlet temperatures on both the load and source side of the heat pump. This test file was then used as the input to conditions to the modelled heat pump. The range of flow rates tested experimentally and compared to the modelled data are as shown in Table 4-1.

Table 4-1: Flow rates for each of the experimental test runs

\begin{tabular}{c|ccc}
\hline Test & Cold Side Fluid & $\begin{array}{c}\text { Hot Flow Rate } \\
(\mathrm{L} / \mathrm{min})\end{array}$ & $\begin{array}{c}\text { Cold Flow } \\
\text { Rate (L/min) }\end{array}$ \\
\hline A & Water & 6 & 9 \\
B & Water & 6 & 6 \\
C & Water & 6 & 3 \\
D & Water/Glycol & 5.5 & 7.5 \\
E & Water/Glycol & 3.5 & 5 \\
F & Water/Glycol & 4 & 4 \\
\hline
\end{tabular}

The performance maps were then implemented into a basic TRNSYS model, with the inlet source and load temperatures and flow rates taken from the experimental data. The outlet temperatures for both sides of the heat pump were then calculated using the model and compared to the experimental results. These results are shown for water on the left of Figure 4-1, and for the water/glycol mixture on the right. 

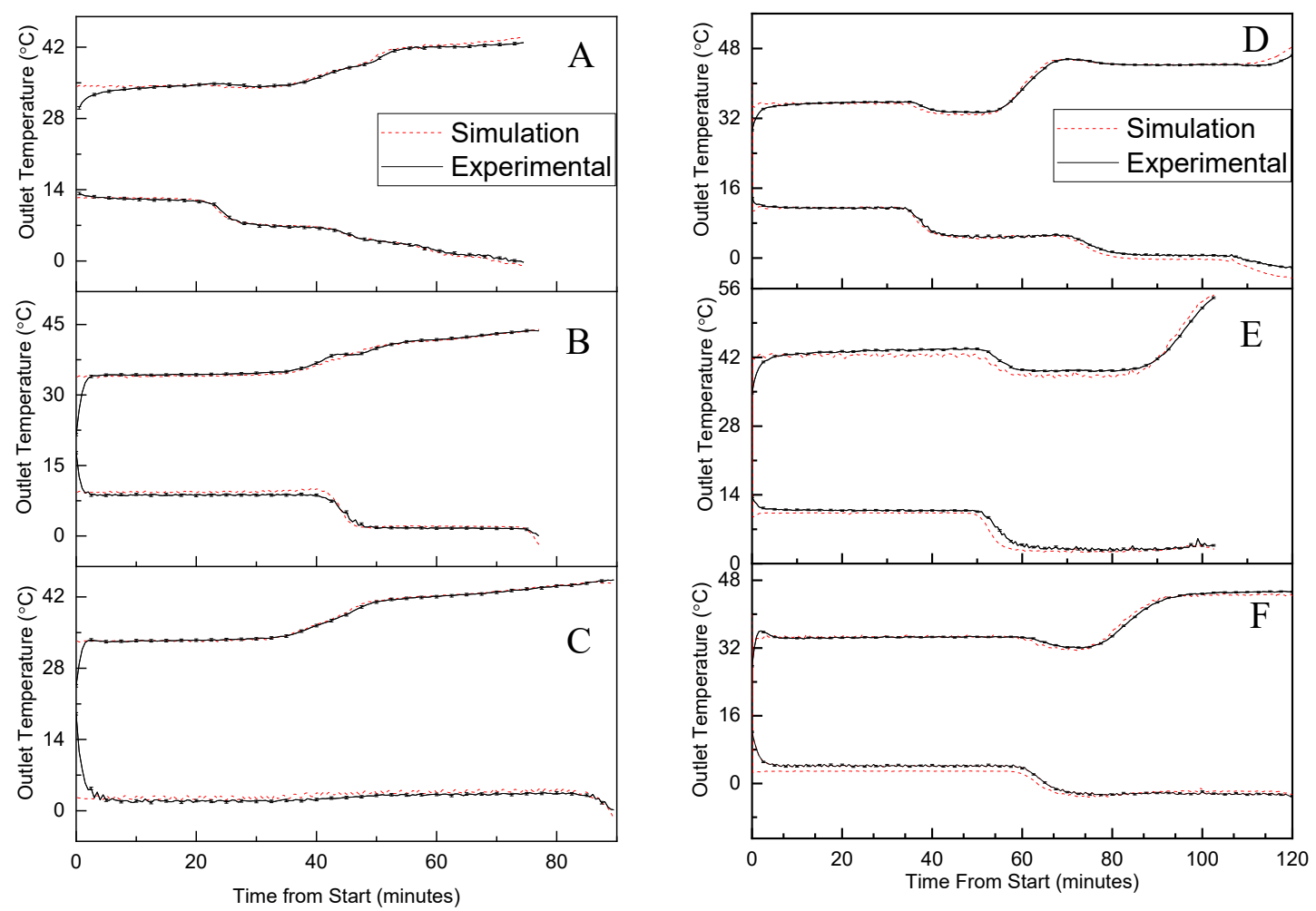

Figure 4-1: Comparison of experimental and simulated performance of the heat pump with water (left) and water/glycol solution (right) with flow rates as indicated in Table 4-1

Using this result, an analysis of the output temperatures was conducted to ensure the model accurately represented the heat pump performance. When looking at the outlet temperatures, it was found that there were little to no discrepancies between the experimental and modelled data. There is a small discrepancy between the two data sets near the end of the test, which is at the boundary of the performance map, and if the inlet conditions are outside the temperature bounds of the performance map, errors in the predicted performance can be observed. Typically, this results in an over-estimation of the performance of the heat pump when the source temperature goes below the bounds, or the load temperature goes above the temperature bounds. This proves that the heat pump model with the experimentally derived performance maps accurately represents the performance 
of the heat pump, as long as the inlet temperatures stay within the performance map bounds.

Although this is true over most of the test, the steady state model does not account for any initial transient conditions at start-up. The modelling of this initial start-up has been explored in depth by Brideau et al. [74], in which they found that this start up can account for an over prediction of performance in a standard heat pump application when steady state models are used. As the system being evaluated is designed to turn on and work continuously for an extended period of time charging the tank, it was noted that each of the 6 test ranged from 70 minutes to 120 minutes, this initial discrepancy is negligible as it accounted for almost none of the total energy transfer that occurred within the test. Based on these results, it was shown that the temperature profiles developed by the heat pump accurately represent the performance of the heat pump, but the heat transfer rates and power consumption had to also be validated.

To do this, the results from Tests B and E of the previous experiments (Figure 4-1) were further analyzed to determine the load side and source side heat transfer rate. The instantaneous and cumulative heat transfer rates calculated both experimentally and from the modelled result are presented in Figure 4-2 and Figure 4-3. Based on these results it was shown that the experimental and modelled data matched for both the instantaneous heat transfer rates and power consumption, as well as the cumulative energy over the length of the 80-minute test run. At the end of the test, the largest deviation was in the cold side energy transfer, which had a difference of $0.058 \mathrm{kWh}$, or approximately $1 \%$ of the total heat transfer, and well within the experimental uncertainty of the system. 


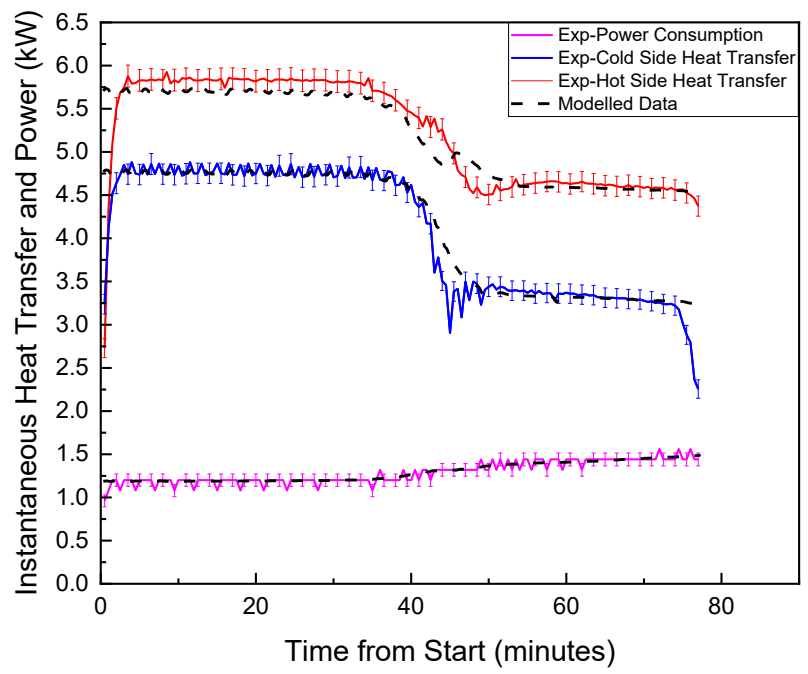

Figure 4-2: Comparison of instantaneous heat transfer rates and electrical consumption - Water

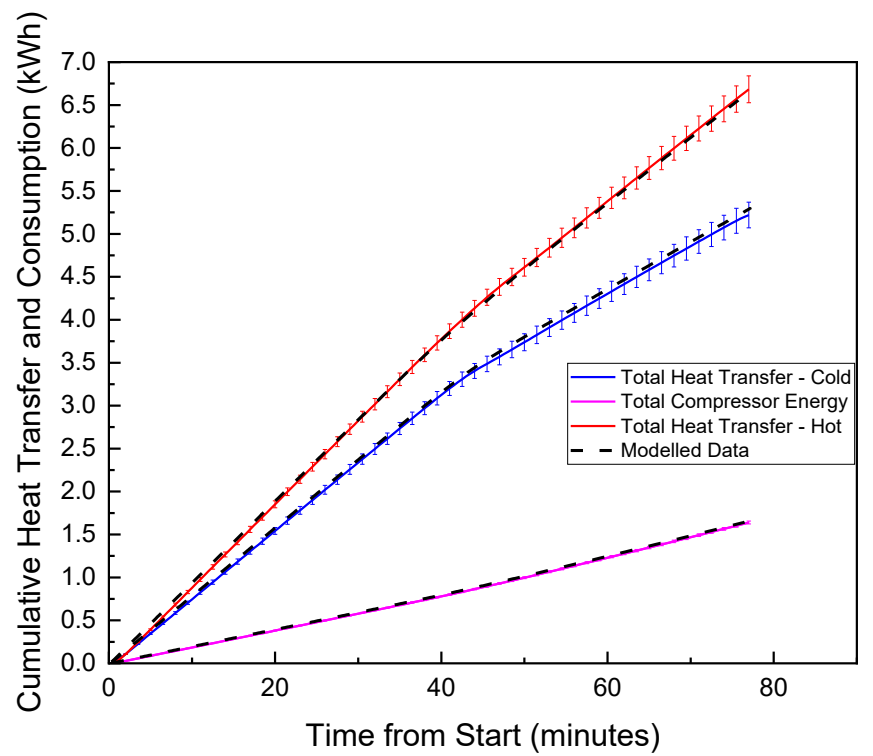

Figure 4-3: Comparison of cumulative heat transferred and electrical consumption - Water

This procedure for validating the heat pump model was then repeated to validate the model when the source (cold) side of the heat pump uses a 50/50 water/glycol solution. The results for this validation test for instantaneous and cumulative heat transfer and 
compressor consumption are shown in Figure 4-4 and Figure 4-5.

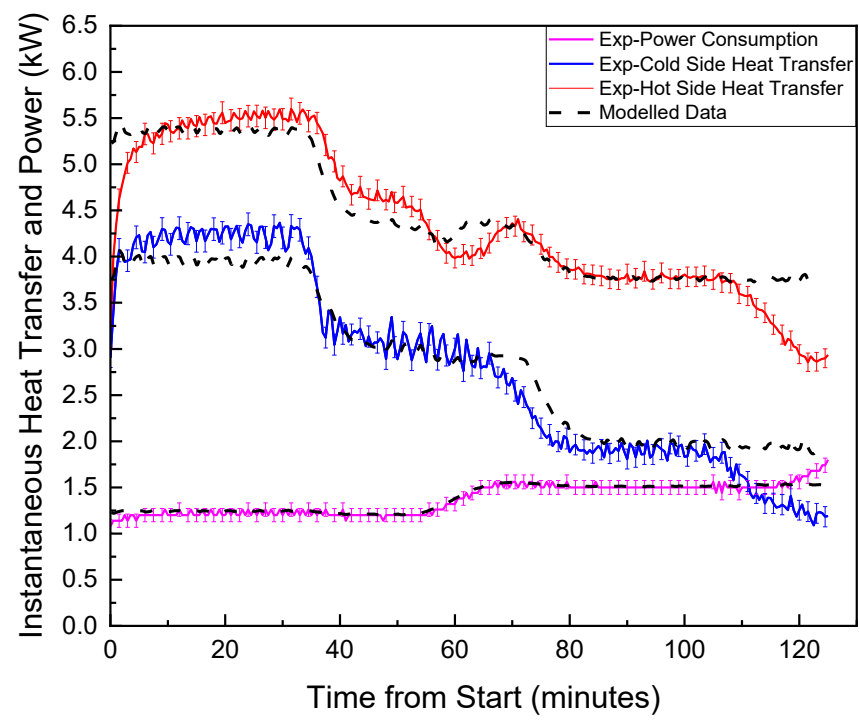

Figure 4-4: Comparison of instantaneous heat transfer rates and electrical consumption -

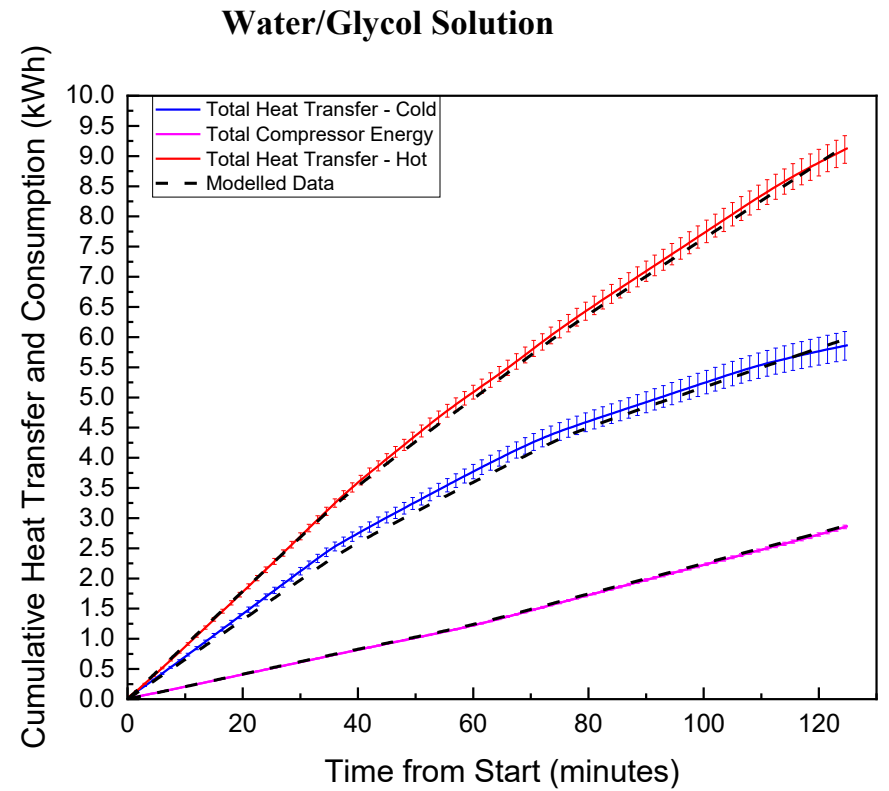

Figure 4-5: Comparison of cumulative heat transferred and electrical consumption Water/Glycol Solution

From these results, the instantaneous values are not nearly as accurate as those seen when modelling the system with water. This shows more unpredictability in the instantaneous performance of the system when using the water/glycol solution, potentially 
due to the reduction in performance of the heat pump seen as a result of the switch from water. In particular, there is a very significant deviation in instantaneous values near the end of the test. This reiterates the additional limitation in the TRNSYS type and its builtin interpolation program; if the input conditions rest outside the provided performance map, the program does not extrapolate, and instead uses the conditions on the edge of the performance map that most closely match the data point. In this case, the entering water/glycol solution on the cold side dropped below $5^{\circ} \mathrm{C}$ and the entering water on the hot side exceed $35^{\circ} \mathrm{C}$, creating a scenario where the predicted performance in far greater than the experimentally measured performance. This result showed it is imperative to keep the test within the range of the developed performance map, and if a significant portion of the simulation occurs outside the performance map range, the performance map must be increased to include the bulk of the simulation time steps. As such, while performing annual simulations, it was imperative that the control systems kept the supply temperatures within the range of the performance map. This was achieved through putting limits on the maximum and minimum temperatures that could achieved in the thermal storage tanks.

Although the instantaneous values have some discrepancies as previously discussed, the total, cumulative heat transfer and compressor energy consumption over the length of the test have a much high accuracy. The total simulated energy remains within the experimental uncertainty for both heat transfer rates as well as the compressor power. Based on this series of experiments and modelled results, it was concluded that the heat pump model using Type 927 and the experimentally developed performance map is suitable to model the performance of the system when using both water and a water/glycol solution on the source (cold) side of the heat pump. 


\subsubsection{Modelling the Medium Temperature Chiller}

The second phase of this project examined the potential for using a medium temperature chiller to provide both the space cooling requirements (using the evaporator side of the chiller) and the space heating requirements (through the condenser side of the chiller). This allows for lower temperatures on the evaporator side of the chiller when compared to the standard heat pump previously tested, meaning it can operate with low source temperatures when heating or reach lower temperatures when storing cooling potential. Although called a "chiller", this operates in exactly the same way the previously modelled liquid to liquid heat pump. The only change being that the cycle cannot be reversed, and therefore the cold side and hot side always remain the same components. As such, the switch between heating and cooling modes had to occur outside of the refrigeration cycle. The experimental unit had a nominal cooling capacity of $12 \mathrm{~kW}$ with a $4 \mathrm{~kW}$ compressor and used R-404a as a refrigerant. This chiller was modelled the same way as the first heat pump, using two Type 927 components, one for the cold side heat transfer rate and compressor power, and a second component to model the hot side heat transfer rate. This was required as Type 927 assumes an ideal system with no losses, and when the performance for the chiller was analyzed, significant loss within the chiller system were observed. This is because the chiller was designed only for cooling, so the condenser is not insulated like the standard heat pump, and therefore a significant portion of the heat transferred through the condenser is lost to the space. A performance map was experimentally developed using the test set-up and formatted for use within the TRNSYS model.

The same validation procedure was used as the standard heat pump to ensure the 
model accurately represented the performance of the chiller. The first parameters which were compared were the outlet temperatures on both the hot and cold side of the system. The plotted outlet temperatures for both the experimental values and the modelled values across three separate tests are shown in Figure 4-6. The hot side was kept constant at $18 \mathrm{~L} / \mathrm{min}$, while the cold side flow rate was A) $9 \mathrm{~L} / \mathrm{min}, \mathrm{B}) 12 \mathrm{~L} / \mathrm{min}$ and C) $6 \mathrm{~L} / \mathrm{min}$.

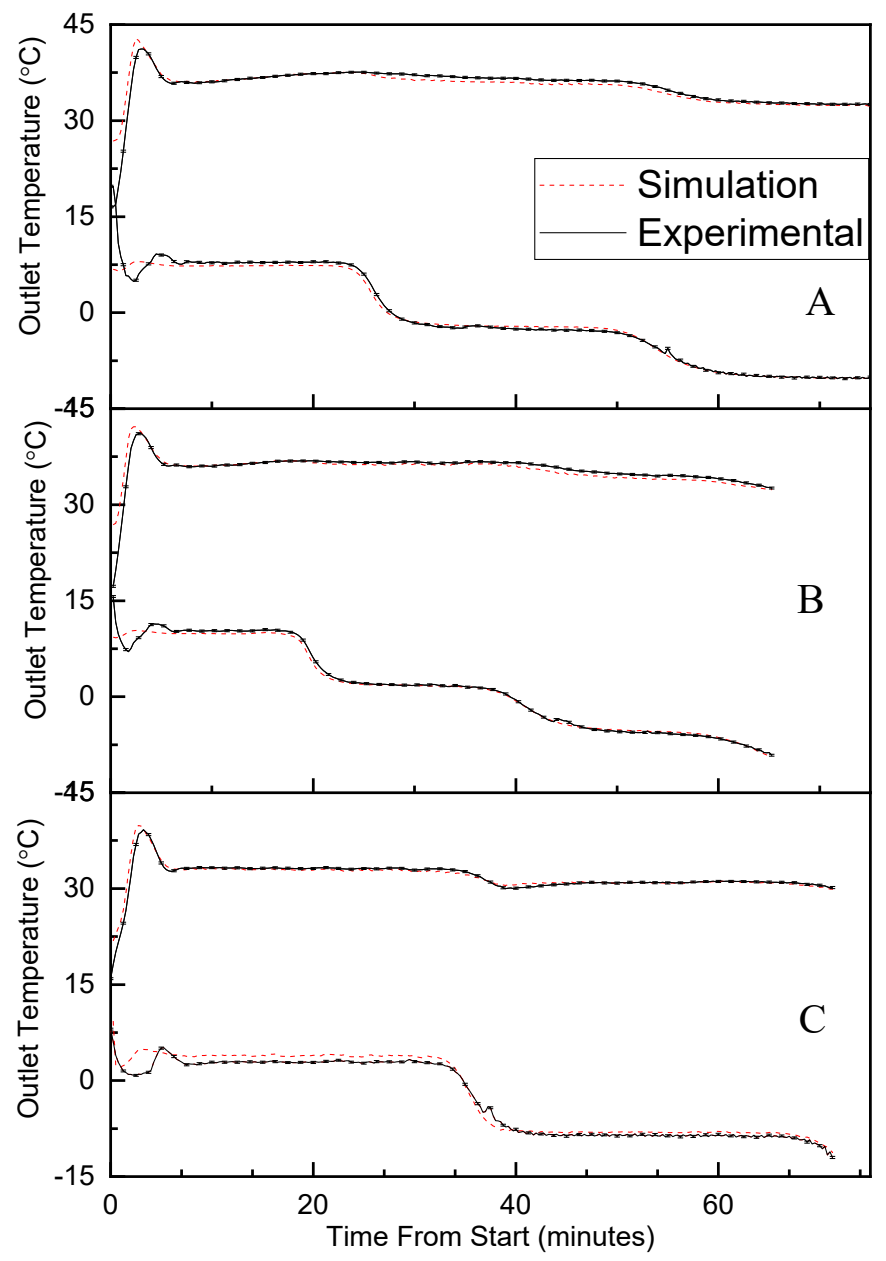

Figure 4-6: Comparison of outlet temperatures for the medium temperature chiller at A) $9 \mathrm{~L} / \mathrm{min}$, B) $12 \mathrm{~L} / \mathrm{min}$ and C) $6 \mathrm{~L} / \mathrm{min}$

These result show that the model accurately predicts the outlet temperature of the medium temperature chiller for both the hot and cold side of the chiller across the complete temperature range the chiller will experience. The next step was to validate the heat transfer 
rates and power consumption, as well as the total heat transfer and energy consumption across the length of the test. To do this, the results from Test B were further evaluated for to ensure the model accurately predicts these values when compared to the experimental values. The comparison between the experimental and simulated instantaneous results are shown in Figure 4-7, while the cumulative results are shown in Figure 4-8.

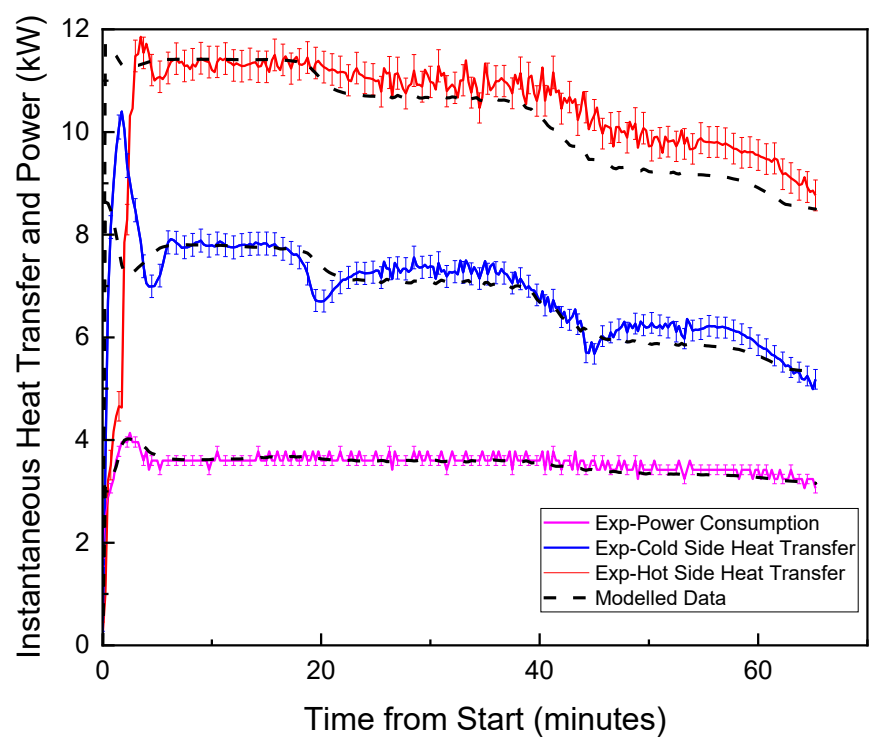

Figure 4-7: Instantaneous heat transfer and electrical consumption for the chiller

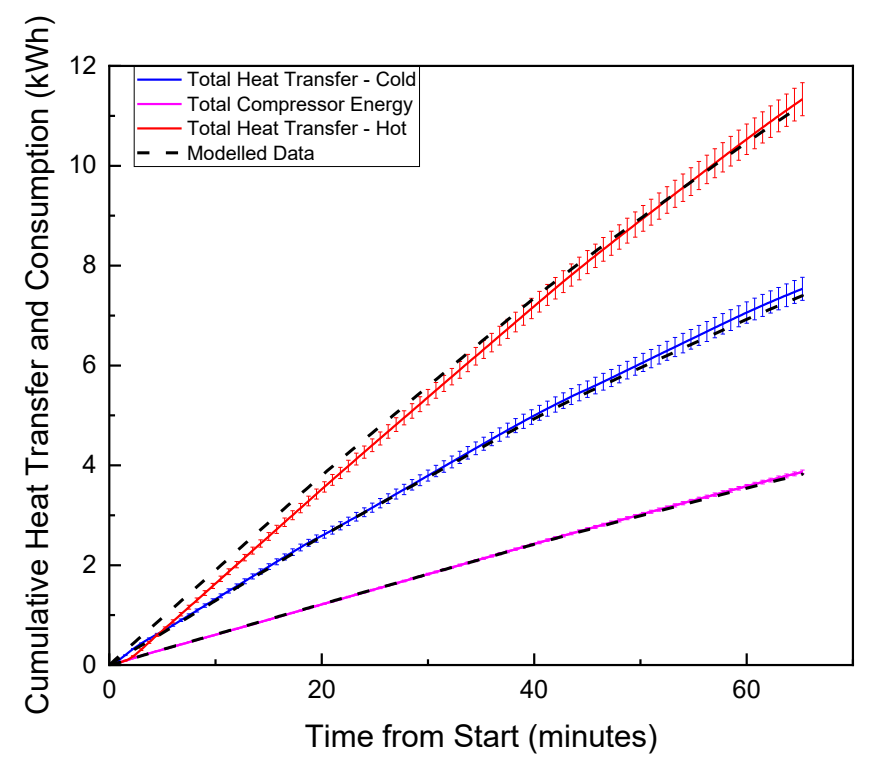

Figure 4-8: Cumulative heat transfer and electrical consumption for the chiller 
From these graphs, it can be seen that there are discrepancies in the initial start-up of the system experimentally compared to the modelled system. This discrepancy is very noticeable in the instantaneous values, however, has minimal impact on the overall performance of the system over the course of the 70-minute test. Additionally, there is a large amount of noise within the experimental data, as a result of the constant changing of the flow rates caused by the PID controller attempting to keep a constant flow as the fluid properties change as the temperature changes. Overall, across the entire length of the test, the total energy transfer modelled is well within the experimental uncertainty of the test system. Based on this, it was determined the model provided the required accuracy for the extended length runs that will be modelled for this system.

\subsection{Modelling Sensible Cold Thermal Storage Tanks}

The initial design of the system to achieve demand side management within the residential setting consisted of two, sensible storage tanks. The first was a hot storage, connected to the condenser side of the heat pump/chiller and the second was a cold thermal storage tank connected to the evaporator side of the heat pump/chiller. The hot thermal storage was designed to use water as the storage medium, while the cold thermal storage will be tested first with water, and then a water/glycol solution. The water/glycol solution was tested to determine whether higher storage densities could be achieved, as the temperatures in which the fluid can be stored can be much lower when using glycol due to its lower freezing point. Additionally, one of the objectives of this work was to determine 
whether the use of a stratified cold thermal storage could be used to improve the performance of the heat pump or chiller being used to charge the system.

To date, almost all research on stratified thermal storage tanks has been completed using hot thermal storage tanks, with little work completed on cold thermal storage tanks. To model both the hot and cold stratified thermal storage tank, TRNSYS was utilized. This allowed the tank as a component to be modelled, as well as the overall interaction between the thermal storage systems and the heat pump, and the heating and cooling system of the modelled house. Within TRNSYS, three different Types have been previously developed and are available to model stratified thermal storage tanks: Type 4, Type 60 and Type 534 (TESS Libraries). These all use similar configurations, where the tank is split up into a predetermined and selected number of nodes, evenly spaced along the vertical dimension of the storage tank and encompassing the entire area of the tank. Each of these nodes then interact with, and transfer heat and fluid between their neighboring nodes, as well as through to the surroundings through heat loss (or gain) across the walls of the storage tank. A more detailed review of modelling methods of thermal storage tanks is provided in Section 2.5.1.1.

The same TRNSYS Type was used for both the hot and cold thermal storage tank, however, many studies were found that validated the performance of the tank model when storing hot water. Through the literature review, no previous studies utilized or validated these TRNSYS Types when the tank is being used to store cooling potential, nor when using a water/glycol mixture as the storage medium. As such, validation of the cold storage 
tank models had to be undertaken to ensure they properly predict the temperature profile and energy storage within the tank.

The first step of the validation process was to define the material properties and physical parameters of the thermal storage system and the material properties of the storage medium. One of the challenges was to select the properties that best represented the material properties, where they could be changing across the length of the test as the temperatures of the changed. As such, properties were selected at the average expected temperature of the material across the test period. The material storage properties of both water and the 50/50 water/propylene glycol solution are provided in Table 4-2 [75, 13].

Table 4-2: Fluid properties of the two storage mediums

\begin{tabular}{l|ll}
\hline Fluid Property & Water & $\begin{array}{l}\text { Water/ } \\
\text { Glycol }\end{array}$ \\
\hline Density $\left(\mathrm{kg} / \mathrm{m}^{3}\right)$ & 1000 & 1046.7 \\
\hline Thermal Conductivity $(\mathrm{W} / \mathrm{m} \cdot \mathrm{K})$ & 0.594 & 0.378 \\
\hline Specific Heat $(\mathrm{kJ} / \mathrm{kg} \cdot \mathrm{K})$ & 4.19 & 3.32 \\
\hline Viscosity $\left(10^{-3} \mathrm{~Pa} \cdot \mathrm{s}\right)$ & 1 & 5 \\
\hline Thermal Expansion $(1 / \mathrm{K})$ & 0.00026 & 0.000495 \\
\hline
\end{tabular}

In addition to the storage medium properties, the tank properties for the cold thermal storage tank must also be inputted to the model. For the validation experiments, the test set-up used a $270 \mathrm{~L}$ standard hot water tank, connected to the source side of the heat pump to provide the cooling source. The tank and the model had the following parameters as outlined in Table 4-3. 
Table 4-3: Physical tank parameters

\begin{tabular}{c|c}
\hline Tank Parameter & Water \\
\hline Volume & $270 \mathrm{~L}$ \\
\hline Height & $1.3 \mathrm{~m}$ \\
\hline Number of Nodes & 50 \\
\hline Fluid Entry Point & Bottom Node \\
\hline Fluid Exit Point & Top Node \\
\hline Auxiliary Heater & $0 \mathrm{~kW}$ \\
\hline
\end{tabular}

These parameters were used for all of the sensible thermal storage validation tests and the corresponding simulation runs. Fifty nodes were initially selected for these models as TRNSYS has a built-in limit of 100 differential equations across the complete model. Each node is represented by a differential equation, and in the complete modelled system, two thermal storage tanks would be present, so 50 nodes was selected as the maximum allowable for each tank, if an equal number of tank nodes were present in each of the modelled tank. The assumption and selection of 50 tank nodes was validated within this process and is discussed in detail in Section 4.2.4.

\subsubsection{Comparison of TRNSYS Model Types}

Once the physical system parameters and material properties were determined, the first test that was conducted was to determine which of the three TRNSYS Types best represented the performance of the cold thermal storage tank. To do this, the same parameters and inputs were placed into a model using each of the Types, and the results were compared graphically using both water (Figure 4-9) and the glycol/water solution as the storage medium (Figure 4-10). 


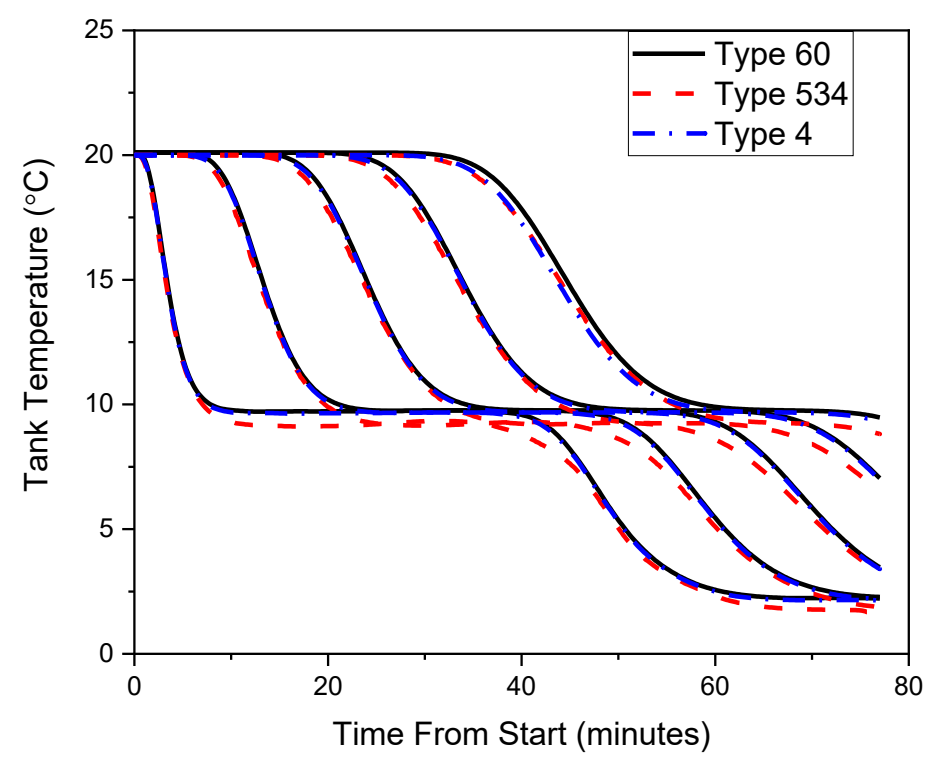

Figure 4-9: Comparison between TRSNYS Types with water as the storage medium

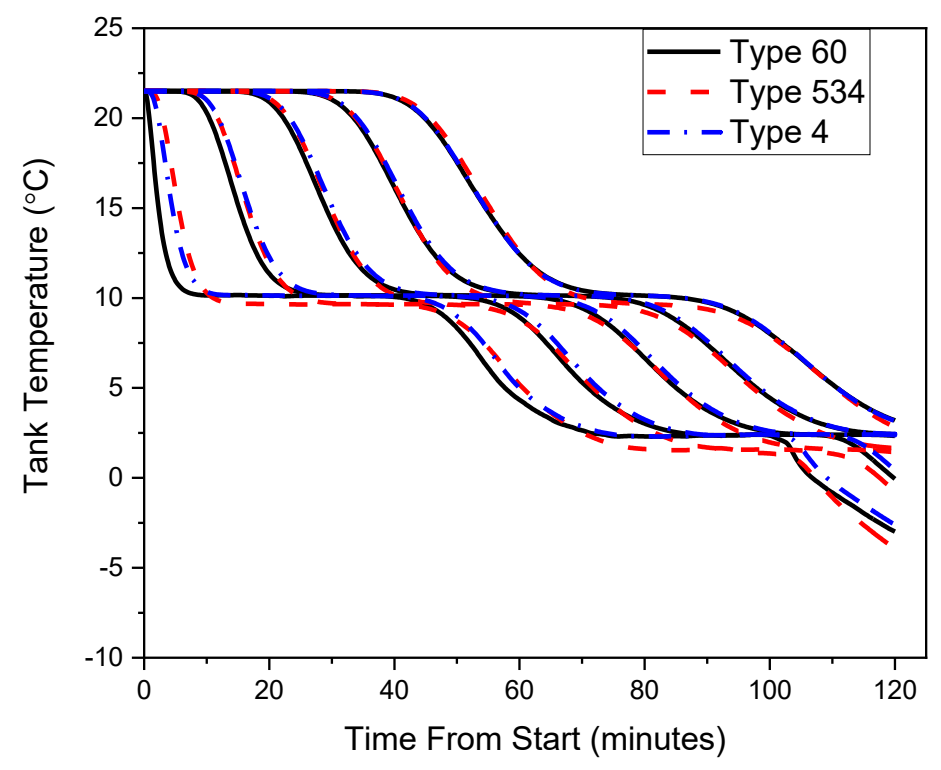

Figure 4-10: Comparison between TRSNYS Types with a water/glycol solution as the storage medium

Based on these results for both storage mediums, all three tank models produce basically identical results, with little to no temperature deviation between each other. What deviations that do occur are predominantly caused by small differences in how the nodes are defined and did not change the overall amount of energy storage within the tank. As 
such, there is no performance benefit between any of the three models and selecting which to use comes down to personal preference and the model in which the modelling of inlet and outlet, heating coils, and internal heat exchangers best represent the physical system. Based on the required components going forward within this study and the added features of the model, Type 534 was selected for the remainder of the validation, and once validated, for use within the larger system and house models.

\subsubsection{Validation of the Cold Thermal Storage with Water as a Storage Medium}

To validate the applicability of the standard TRNSYS thermal storage tank models for use as a cold thermal storage tank with both water and a water/glycol solution, the results using Type 534 were first compared to experimental results. The primary purpose of the comparison was to determine whether the tank model could accurately predict the stratification present during a charging cycle of the cold storage. As such, the temperatures were measured at 13 equal increments through the tank, while the temperature at that level was also determined through simulation. The results were then plotted allowing the temperature profiles to be compared. This process was conducted at three different source side flow rates, allowing the effect of flow rate on both stratification and the ability of the model to predict the temperature profile to be determined. As such, low ( $3 \mathrm{~L} / \mathrm{min})$, medium (6 L/min) and high $(9 \mathrm{~L} / \mathrm{min})$ source side flow rates were compared, with the results in Figure 4-11, Figure 4-12, and Figure 4-13, respectively. In all of these graphs, the left most line represents the bottom most measurement point moving up the tank to the right to the graph with the right most line representing the top measurement point. The first measured point is located $5 \mathrm{~cm}$ from the bottom of the tank, and in $20 \mathrm{~cm}$ increments with the top measurement being $125 \mathrm{~cm}$ from the bottom of the tank. 


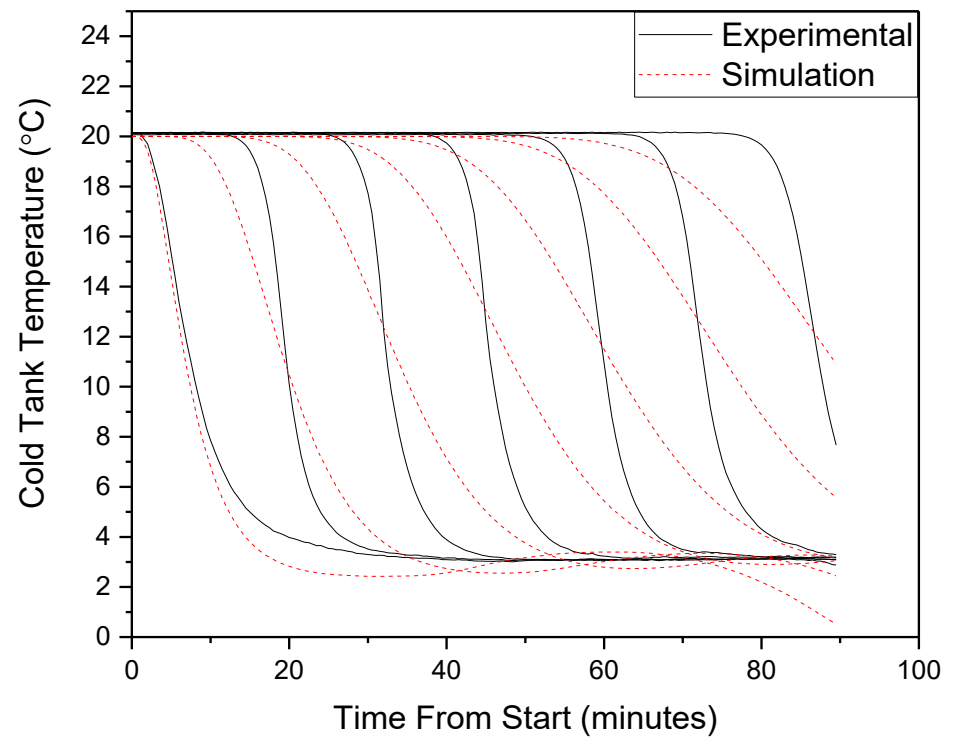

Figure 4-11: Comparison of tank profiles at a flow rate of $3 \mathrm{~L} / \mathrm{min}$

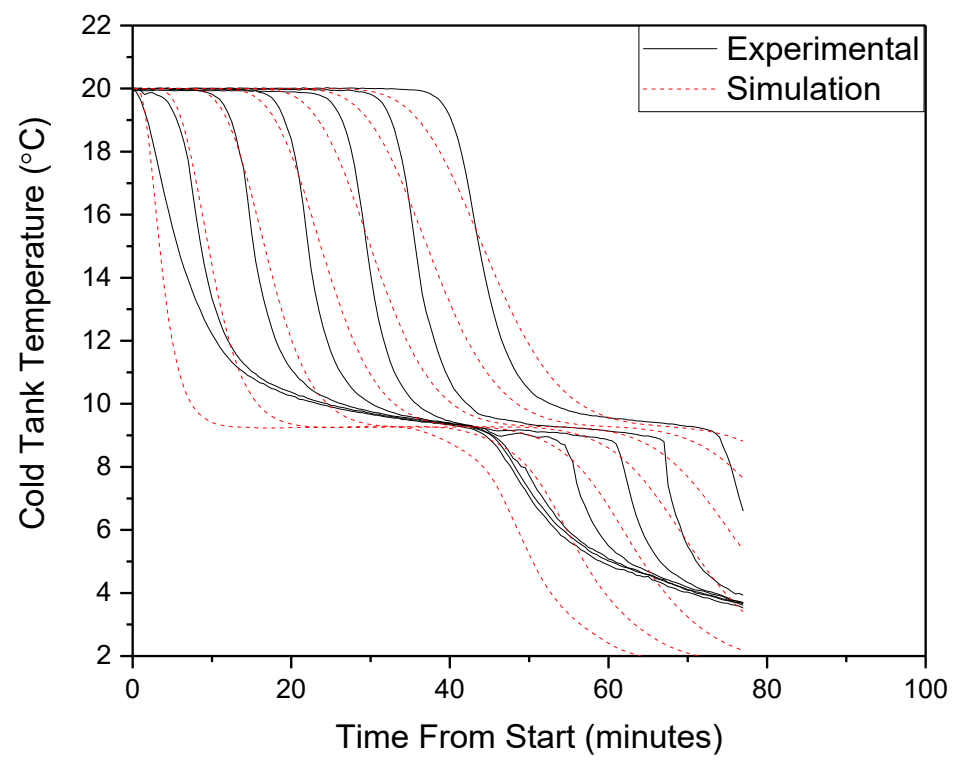

Figure 4-12: Comparison of tank profiles at a flow rate of $6 \mathrm{~L} / \mathrm{min}$ 


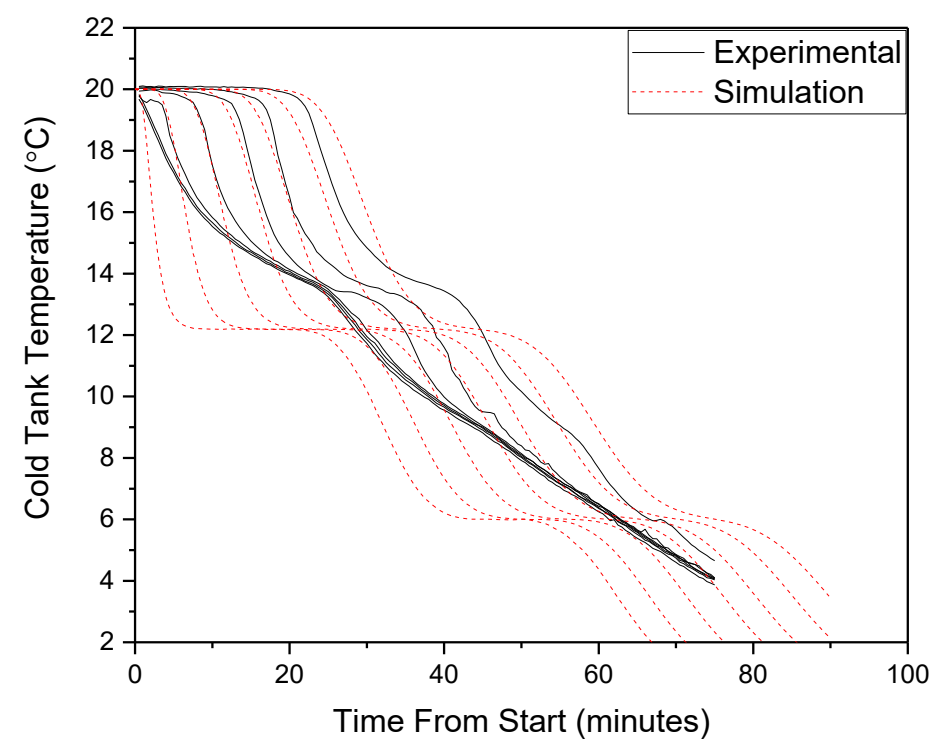

Figure 4-13: Comparison of tank profiles at a flow rate of $9 \mathrm{~L} / \mathrm{min}$

From these figures, it can be seen that as the flow rate increases, the experimental results show a rapid destruction of stratification starting at the bottom of the tank (where the inlet is located). Additionally, as the temperature of the tank storage medium decreases, the stratification seen in the tank also decreases and as this decrease in temperature occurs, the more the tank resembles a fully mixed tank. As a result, it was determined that the model accurately represents the temperature profile of the tank at low flow rates, and at higher temperatures, however the models fail to accurately predict at high flow rates and as the temperature approaches freezing. This impacted the temperature distribution within the tank, but not the overall energy stored. This is caused by the plume effect, where the inlet acts as a jet entering the tank, causing rapid fluid transfer between nodes as described by Csordas et al. [76]. This is compared to the lower flow rates, where the fluid enters and remains in the entry node, and slow moves up the height of the tank as more fluid is removed. In this case, the only transfer of fluid between nodes is the natural convection occurring between nodes, and no forced flow between the nodes. As the focus of this work 
was on stratified storage tanks at lower flow rates, the models were used as is going forward, however, if higher flow rates were to be tested, model parameters that control the flow of fluid between nodes would have to be further examined.

\subsubsection{Validation of the Cold Thermal Storage with a Water/Glycol Solution as a}

\section{Storage Medium}

This process was repeated, but the storage medium was replaced with a 50/50 water/glycol solution (by volume). The simulations were run using Type 534, however all the fluid properties were changed to those of the glycol solution. The simulations were run at a low $(4 \mathrm{~L} / \mathrm{min})$, medium $(5 \mathrm{~L} / \mathrm{min})$ and a high $(7.5 \mathrm{~L} / \mathrm{min})$ source side flow rate. A slight change in flow rates from the water tests were used to further validate the models at different flow rates. The simulation and experimental results were plotted together for each set of test conditions and the results are shown in Figure 4-14, Figure 4-15, and Figure 4-16 respectively.

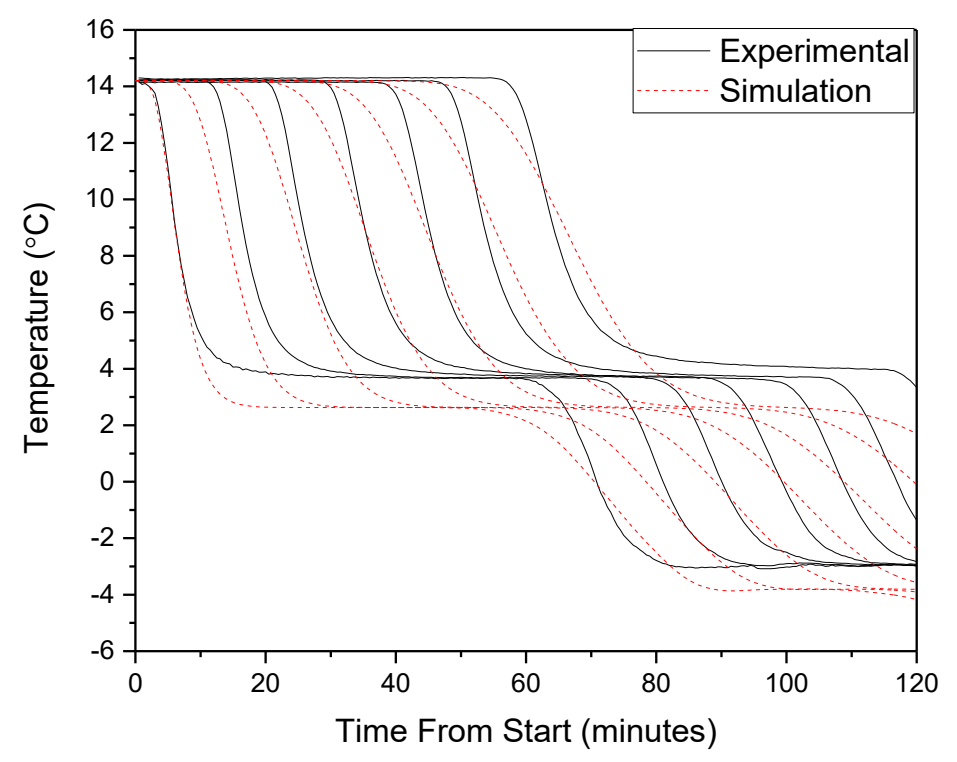

Figure 4-14: Comparison of tank profiles at a flow rate of $4.5 \mathrm{~L} / \mathrm{min}$ 


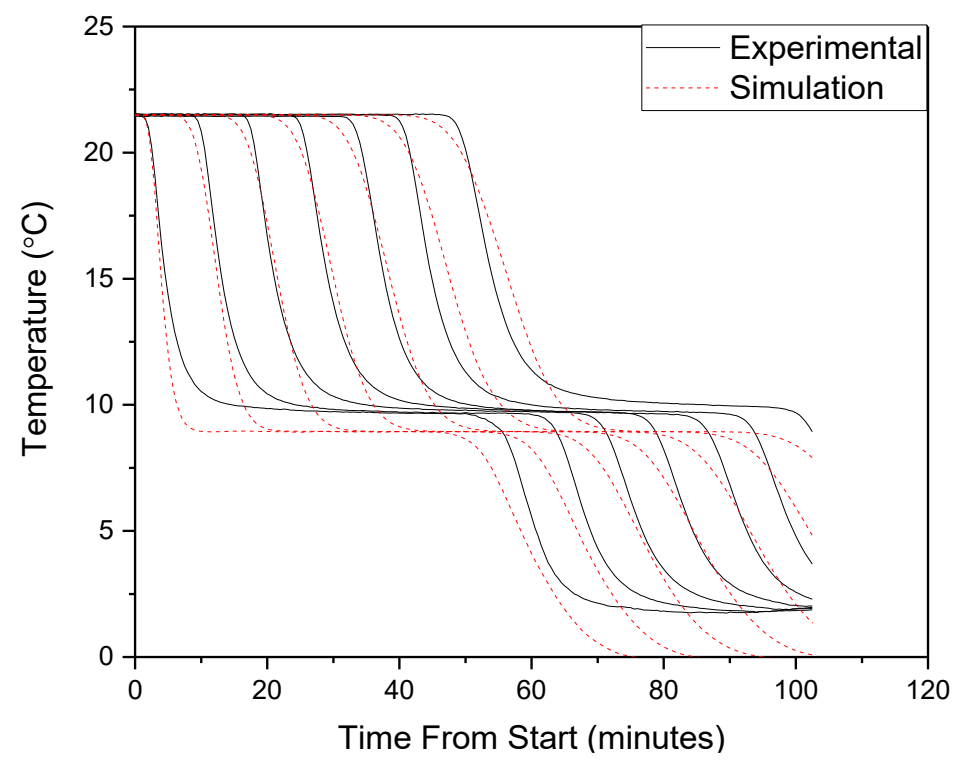

Figure 4-15: Comparison of tank profiles at a flow rate of $5 \mathrm{~L} / \mathrm{min}$

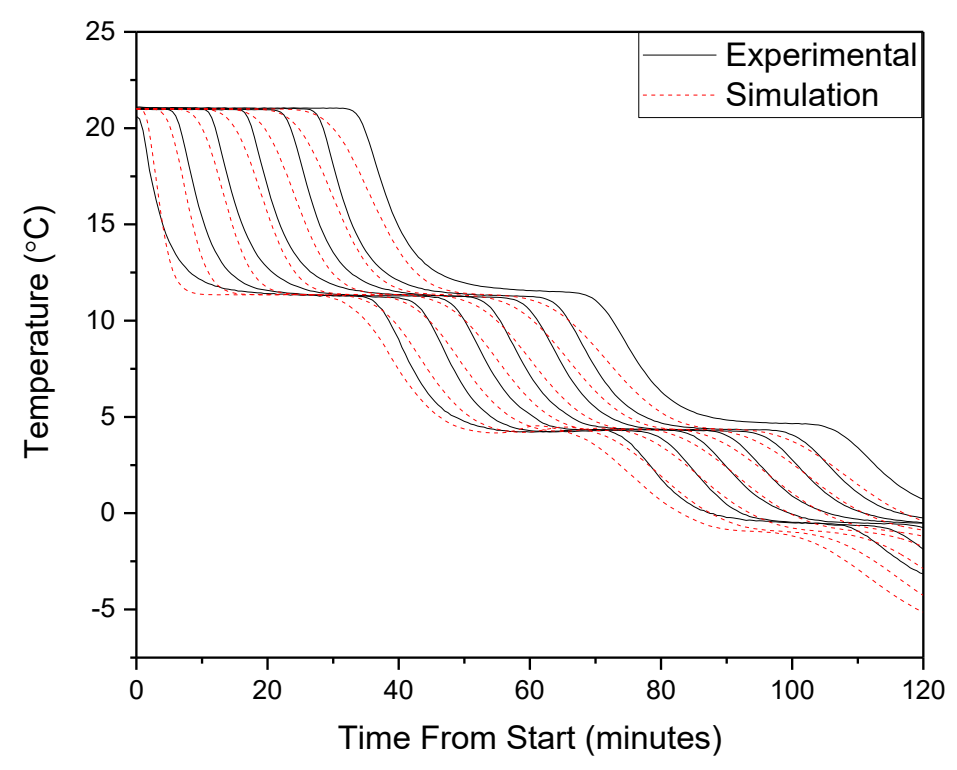

Figure 4-16: Comparison of tank profiles at a flow rate of $7.5 \mathrm{~L} / \mathrm{min}$

Based on these results, it is shown that TRNSYS Type 534 adequately predicts the temperature distribution within a cold thermal storage system that uses a glycol/water solution as the storage medium. A small discrepancy is noted in the end temperatures, with this most likely resulting from small discrepancies seen in the source outlet temperatures of the heat pump. There is no change in the predictive capabilities of the model at higher 
flow rates or at lower tank temperatures as seen in the experimental results of the tank temperature profiles when using water as the storage medium.

The simulation and experimental results have almost a perfect agreement at the beginning of the test, however as the test continues, the level agreement between the experimental and simulation results slowly decreases. This is caused by the small errors introduced through each component and time step slowly building on each other and compounding, as a small difference in entering heat pump temperature can cause a larger error on the outlet temperature and then compounds within the thermal storage tank temperature profile.

\subsubsection{Node Sensitivity Analysis}

In the storage tank when both water and a water/glycol solution are used as the storage medium, the experimental measurement changes temperature at a much greater rate than the modelled values. This is caused by the fact that the experimental set-up measures the temperature at a single point, capturing the change as the storage charges up at that exact point. This differs from the simulated value, in which the temperate of a given node is provided, which requires more time to change as the system is charged. The model works on the premise that the tank is split up into $\mathrm{N}$-number of nodes, where in this case, $\mathrm{N}$ is 50 . Within each node, the temperature is assumed uniform, and since, with 50 nodes, each node represents $\sim 5.4 \mathrm{~L}$ of fluid, the slower change in temperature is a result of the increased time it takes to decrease the temperature of the nodal fluid. This discrepancy between modelled and experimental results has been extensively studied and evaluated in literature, and the only way to decrease the difference in the rate of change of the temperature at a given point is to increase the number of nodes in the tank. Based on the previous work, if the tank was 
divided into an infinite number of nodes, the simulated results would change at the same rate as the experimental values, but due to the practicality of using a large number of nodes and the required computing power, the approximation from these nodes provides the necessary temperature profiles required.

Although it is expected that there is a difference between the nodal temperature and the experimental results as discussed, the true impact on the number of nodes needed to be tested, to determine the impact of increasing or decreasing the number of nodes used to model the storage tank. As such, a sensitivity analysis was completed on the cold tank using both water and water/glycol solution as the storage medium. The tank model was tested using 12 nodes, 25 nodes, 50 nodes and 100 nodes for the cold thermal storage, and the modelling results where then compared graphically to the experimental results in Figure 4-17 and Figure 4-18.

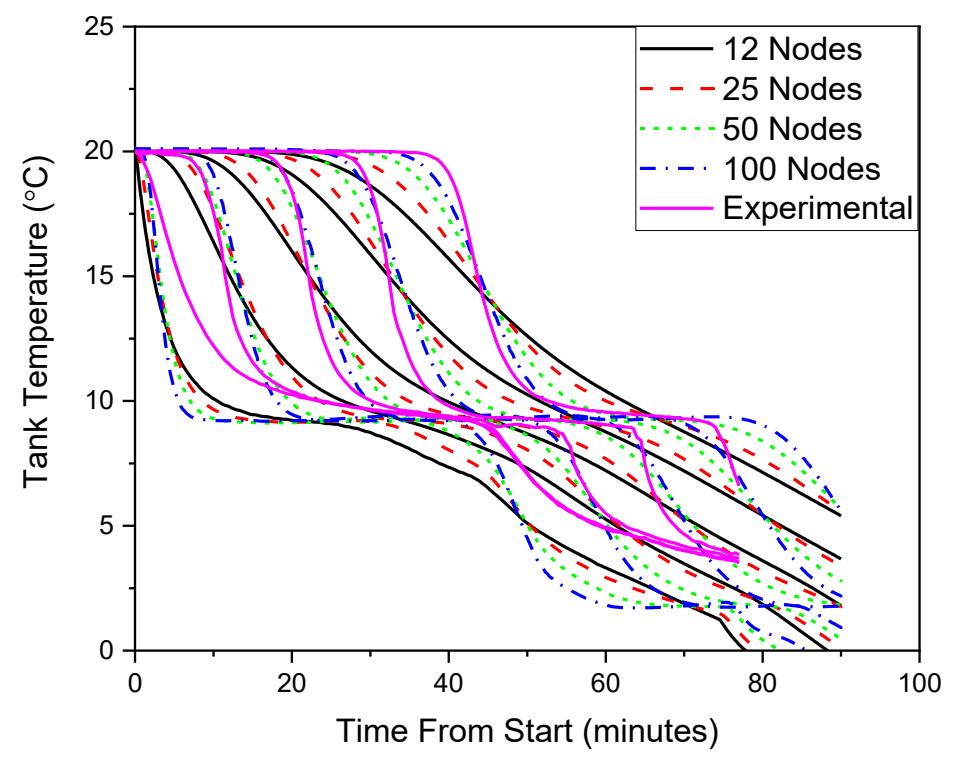

Figure 4-17: Comparison of number of nodes with water as the storage medium 


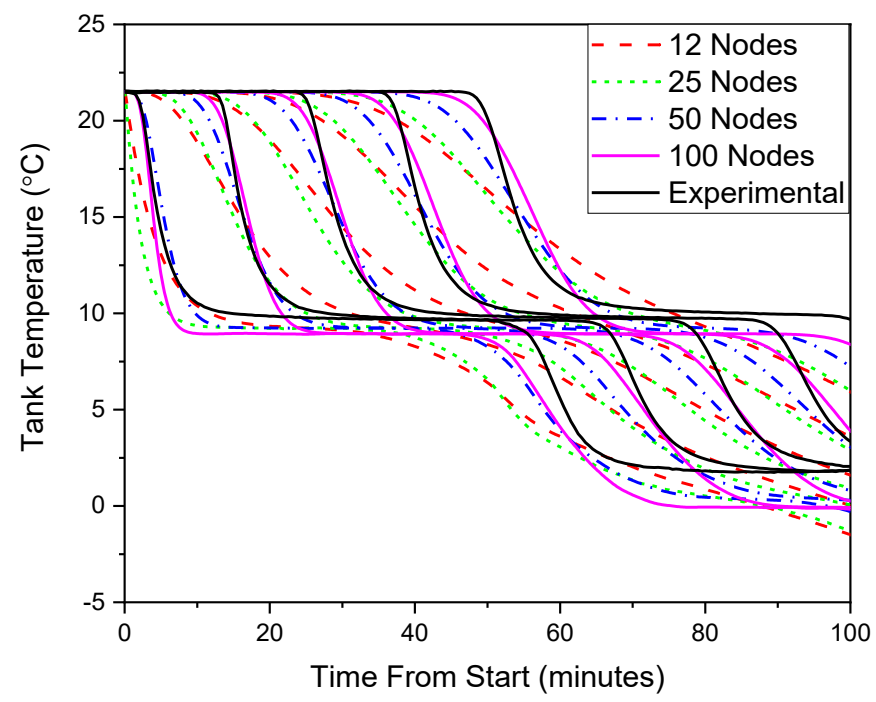

Figure 4-18: Comparison of number of nodes with a glycol solution as the storage medium

These graphs show the expected result where the more nodes in the tank, the quicker the rate of temperature change experienced at any given point. This is most evident when comparing the extremes of the simulation results using 12 nodes when compared to that using 100 nodes. There is also a significant volume per node difference, where a node represents $22.5 \mathrm{~L}$ when using 12 nodes, to $2.7 \mathrm{~L}$ when using 100 nodes. This confirmed that there is a significant improvement in the tank temperature profile as the number of tank nodes increased.

Although it was expected to see a significant difference between the two extremes, of more interest was the difference between 50 and 100 node cases. In both the water and water/glycol cases, there is an improvement going to 100 nodes from 50 nodes, however this improvement is small (particularly when looking at the glycol/water solution). As a result, it can be said that the 50 node thermal storage tank model has the necessary accuracy to model the cold thermal storage tank in the system of interest. This was an extremely important determination, as TRNSYS can only handle a total of 100 nodes across all tanks 
in the model. When the complete system will be modelled with both a hot and cold thermal storage tank, this allows each tank to be modelled with 50 nodes.

\subsection{Modelling Latent Cold Thermal Storage Models - Ice Storage}

As this research project progressed with full building modelling (as discussed in the next two chapters), it was determined that the sensible thermal storage did not have the required thermal storage densities required to shift off-peak cooling loads. As such, higher thermal storage densities were examined, and through this study, it was determined that ice storage would suit the requirements of the application, while having a storage density in excess of four times that of the sensible thermal storages. Within ice thermal storages, a number of different configurations exist, but for this application, an ice-on-pipe system was chosen. This is a relatively simple system, that does not require any sophisticated chiller or control systems and functions by passing chilled water/glycol solutions through pipes submerged in an unpressurized reservoir. The cooling potential transfers through the submerged pipes, cooling the storage medium down, and then once the temperature of the water reaches $0^{\circ} \mathrm{C}$, ice begins to form on the surface of the pipes. Ice continues to grow out radially from the pipe surface, until, in theory all of the water is converted to ice, and maximum storage density is obtained. That being said, in practice, at no point will $100 \%$ of the water be converted to ice, where ice formation is typically seen as between 40 and $60 \%$ of the volume of the water.

To calculate the required capacity of the ice storage, both the sensible cooling within the water as well as the heat of formation of the ice that builds up on the pipes were required. An equation has been derived that uses the volume of water and the percentage of water converted to ice to determine the storage capacity and can also be used during 
operation to determine the amount of cooling potential stored at any point, as shown in Equation (4-1).

$$
E_{\text {Stored }}=m_{\text {water }} c_{\mathrm{p}}\left(T_{\text {avg }}-T_{\text {max }}\right)+I F \cdot m_{\text {water }} \cdot H_{\mathrm{f}}
$$

where $m_{\text {water }}$ is the total mass of water within the storage tank, $c_{\mathrm{p}}$ is the heat capacity of the water, $T_{\mathrm{avg}}$ is the average temperature of water in the tank, $T_{\max }$ is the maximum delivery temperature to the air conditioning coils, $I F$ is the fraction of water in the tank that is ice, and $H_{\mathrm{f}}$ is the heat of formation of water solidifying to ice.

To compare the storage capacity of the ice storage compared to the sensible storage, the required volume of an ice/water tank was determined to obtain the same storage capacity as a $2000 \mathrm{~L}$ water tank. To make this calculation, it was assumed that the fraction of ice is $50 \%$ (i.e. $50 \%$ of the water can be made into ice through storage), and all other water in the tank is at $0^{\circ} \mathrm{C}$. A total mass of water of $446 \mathrm{~kg}$ is required to match the sensible storage capacity, or a final volume of 467 L (446 L of water before solidification). From this, the same quantity of cooling potential can be stored in a volume that is $23.4 \%$ that of the sensible storage system. If ice fractions greater than $50 \%$ can be obtained, the required volume will even be less that the calculated value, however a $50 \%$ value will be used as a conservative benchmark until experimental data proves that a greater ice fraction is obtainable.

To study the potential for ice storage within the larger system, a model for the ice storage had to first either be found in literature or developed. When going through 
literature, it was found that almost all models previously used fell into one of two categories:

1) a lumped capacitance model where all of the cooling potential generated by the source being stored either at $100 \%$ or an experimentally determined storage efficiency (usually around 90\%), or

2) detailed finite element models, using computational fluid dynamics (CFD) or finite element analysis (FEA) solvers to model each element of fluid within the tank.

Both model types had their own advantages and disadvantages. The lumped capacitance models were advantageous as they were very simple models, and did not require much computing power to run on annual simulations, but the assumption had to be made that all of the cooling provided could be stored, and that there was no real interaction between the chiller and the storage. This works for systems that use a slush generator, or encapsulated particles, but there is a direct correlation between how much cooling can pass through the pipes and into the storage medium. Additionally, the return temperature to the chiller must be determined, whereas with the lumped capacitance model, an assumption must be made that the return temperature is either constant, or the temperature of the thermal storage. As such, it was determined that a lumped capacitance model was not suitable for an ice on pipe thermal storage system due to its over simplicity, but also incompatibility between the physical system and its operation, and the assumption required in the model.

Next the detailed FEA and CFD models were examined for their use within the proposed system. Although these systems produce excellent, detailed and accurate results, 
they require significant computing power to solve the CFD/FEA computational model. This is practical when doing detailed design work on a thermal storage system, however, is impractical when modelling performance over extended periods of time. This is the case in this study, where annual performance of a system is studied, modelled at anywhere from 30-second to 5-minute time steps, which requires up to over 1 million individual simulations. As such, it was decided that a CFD/FEA model was would not work for this application, due to either the long simulation time or the computing power that would be required for optimization of parametric studies on an annual basis.

Once the existing models were determined to not be compatible with the requirements of the current research project, alternative modelling strategies were determined. The first idea that was explored was the experimental development of a performance map that determined the heat transfer rate and the ice growth rate given a set of conditions. The independent variables would include the temperature of fluid flowing through the pipe, the flow rate of the fluid through the pipe, the thickness of the ice on the pipe and the average temperature of the tank. This would need to be repeated for both the charging and the discharging of the system. As this modelling method was further explored, a number of issues quickly arose. As the range of possible temperatures both in the pipe and the tank temperatures, and flow rates were determined, the number of points within the performance map required to accurately represent the system was massive, and as such, the experiments could not be feasibly conducted. Additionally, the experimental set-up cannot be operated under steady state conditions, as the thermal storage is constantly charging, and as a result the conditions are constantly changing. This limited what type of experimental performance map could be developed. Finally, this method was not further 
pursued as a full set of experiments would need to be conducted for each pipe configuration, so no changes could be made to the pipe diameter, material or pipe thickness without changing the performance map.

The second new modelling method that was explored was calculating the heat transfer rate through the pipe and ice into the tank, and from that, the tank temperature and the amount of ice formation can be calculated. This method allows for the interaction between the chiller and thermal storage to be determined, any pipe geometry to be modelled without extensive experimental evaluation, and the system can be modelled using a minimal number of nodes, reducing the computational time required for the simulations of annual performance to run. The detailed development of this model for the ice storage is presented in the proceeding section, as well as the experimental validation of this model.

\subsubsection{Model Development}

Once the use heat transfer rates was determined as the selected approach to model the ice storage, and in the application being explored in this study, detailed development of the system and modelling structure was completed. Before the detailed mathematical representation of the system could be coded, the model architecture had to be first developed and the overall system geometry and parameters were determined. The ice storage system consists of a series of pipe submerged within a larger tank, equally spaced throughout the volume of the tank. For this model, a single pipe was modelled in detail, and then, based on the assumption that the heat transfer and ice formation will constant on all pipes, the results will be extrapolated across the total number of pipes. This is because all pipes will have the same inlet conditions, and since the tank is at a constant temperature of $0^{\circ} \mathrm{C}$, the heat transfer and therefore the formation of ice will be constant across all pipes. 
The number of pipes within the volume of the tank is a variable and can be inputted to the model as a parameter. The pipe being modelled in detail is further broken down into 12 nodes, where node 1 is the entry point of the working fluid providing cooling, and node 12 is the exit point of the fluid after the fluid transfers all of its energy to the thermal storage tank. The fluid parameters (temperature, flow) from the chiller enters node 1 , and the conditions of the fluid leaving the preceding node become the entry conditions for the following node, with the exit conditions of node 12 being the return conditions to the chiller. Figure 4-19shows the geometry of the pipes within the tank, while Figure 4-20 shows the node configuration of a single pipe.

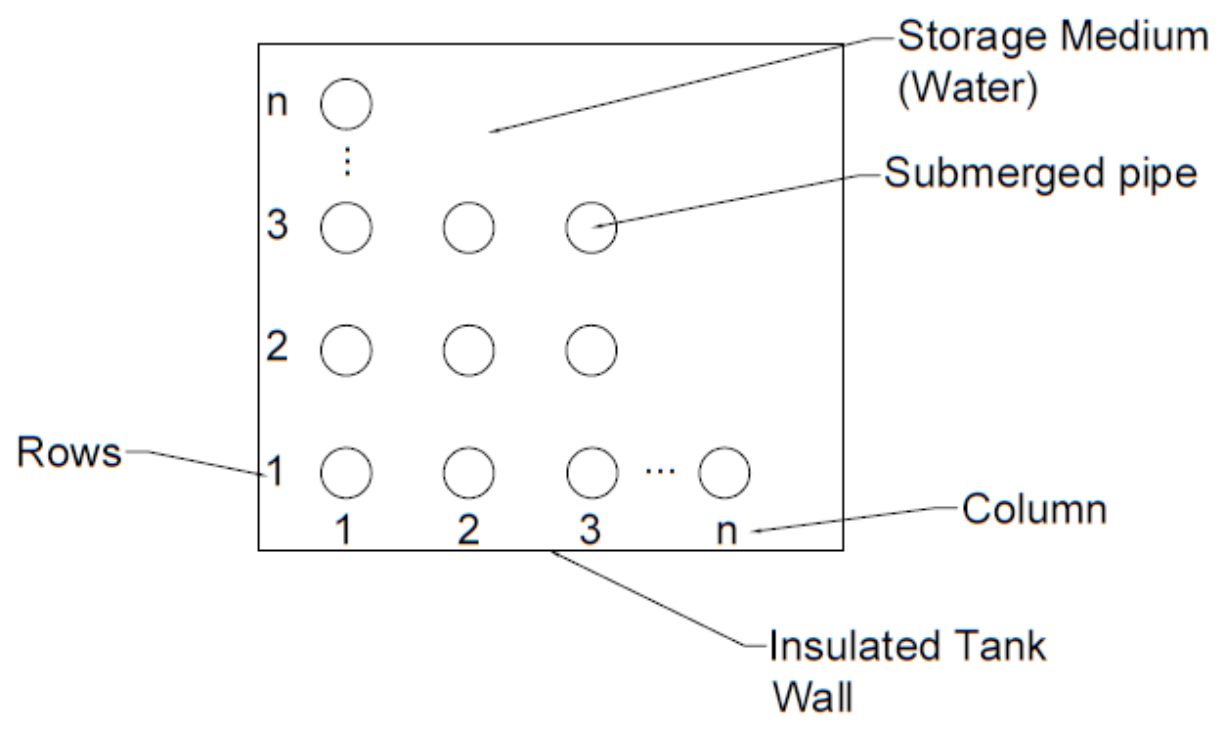

Figure 4-19: Pipe configuration within the ice storage system 


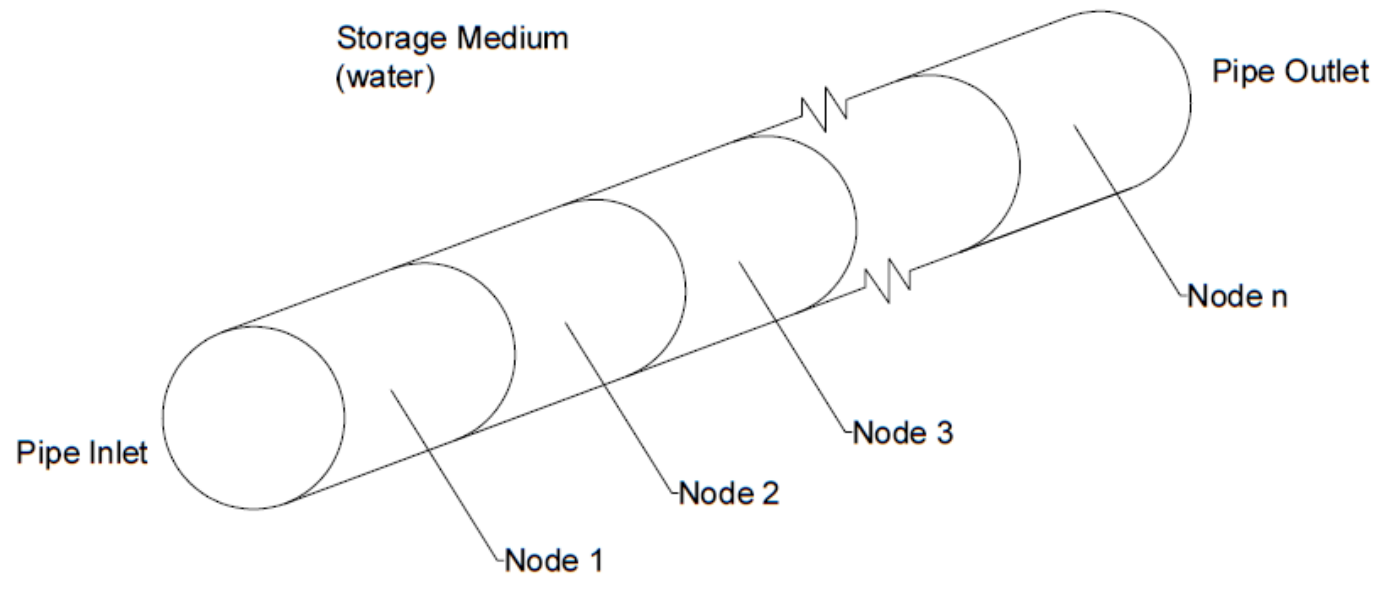

Figure 4-20: Node configuration on a single submerged pipe

To model the pipe in detail, a heat transfer model was developed, where heat is transferred from the moving fluid, through the pipe wall and ice buildup (if ice has already formed), and then into the storage fluid (water). To develop this heat transfer model, a radial coordinate system was utilized for all calculations, where the heat transfer across/at four boundaries was required. These were the forced convection from the moving fluid in the pipe to the pipe material, the conductive heat transfer through the pipe wall, the conductive heat transfer through the ice, and the natural convection from the surface of the pipe/ice to the tank fluid. A schematic of the selected surface, as defined in the overall heat transfer calculations are shown in Figure 4-21, while the detailed mathematical description and governing equations for each location are provided in detail in the proceeding sections. Information was taken from Bergman et al. [77], Moran et al. [13], and Holman [78] to 
derive and develop the complete heat transfer model used to characterize the ice storage system.

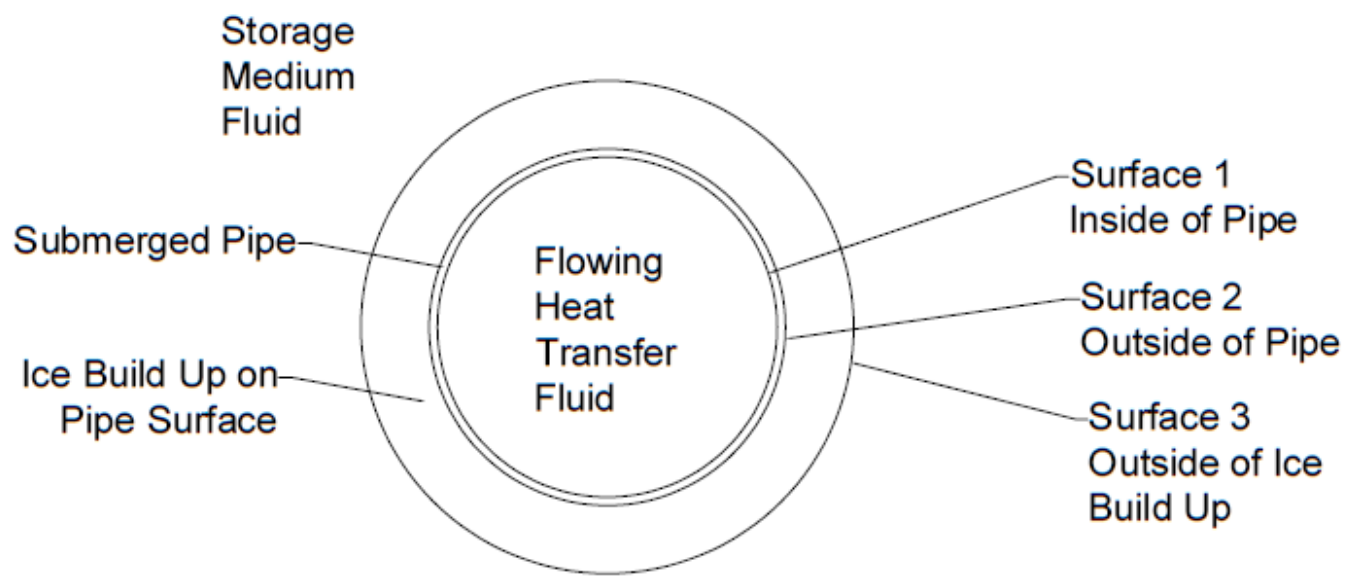

Figure 4-21: Schematic of the submerged pipe, with ice build up, and heat transfer surfaces indicated

\subsubsection{Forced Convection in the Pipe}

A 50/50 glycol/water solution is utilized as the heat transfer fluid, where the chiller reduces the fluids temperature, and providing the required cooling. The chilled fluid passes through the submerged pipe, transfer the cooling potential from the fluid into the storage tank. The first heat transfer that occurs and has to be calculated is the forced convection from the fluid into the wall of the submerged pipes. To calculate this heat transfer, the Reynolds Number of the flowing fluid had to first be determined, using Equation (4-2).

$$
\operatorname{Re}=\frac{V D}{v}
$$

where $V$ is the velocity of the flowing fluid, $D$ is the inside diameter of the pipe, and $v$ is the kinematic viscosity of the flowing fluid.

The forced heat transfer equations are based on whether the flow within the pipe is in the laminar or turbulent range. Using a first assumption where the maximum flow 
velocity is $1 \mathrm{~m} / \mathrm{s}$, which is the equivalent of $3 \mathrm{~L} / \mathrm{min}$ through the test pipe, which has a diameter of $8 \mathrm{~mm}(0.008 \mathrm{~m})$. Finally, the kinematic viscosity of the water/glycol solution was found to be $2.38 \times 10^{-5} \mathrm{~m}^{2} / \mathrm{s}$, based on a dynamic viscosity of $0.025 \mathrm{~kg} / \mathrm{ms}$ and a density of $1050 \mathrm{~kg} / \mathrm{m}^{3}$. Using these three values, a Reynolds Number of 336 was determined for this situation, which is in the laminar range (for pipe flow, less than 2000). This showed that even if the diameter of the pipe was increased, as long as the pipe did not increase to greater then $\sim 48 \mathrm{~mm}$, while maintaining the same flow velocity, which if assumed to be $1 \mathrm{~m} / \mathrm{s}$, would equate to a flow rate of $108.5 \mathrm{~L} / \mathrm{m}$. Based on these assumptions, it was determined that for all applications within the ice storage system, a laminar pipe flow would be expected and only the equations for laminar flow will be considered within the model. As such, one limitation of this model is the fluid flow through the submerged pipes must be laminar for the model to be valid.

To calculate the overall heat flux as a result of the forced convection, the general convective heat transfer equation is utilized, as shown in Equation (4-3).

$$
q=h_{\mathrm{c}} A\left(T_{\mathrm{G}}-T_{1}\right)
$$

where $q$ is the heat transfer from the fluid to the surface, $h_{\mathrm{c}}$ is the convective heat transfer coefficient, $A$ is the heat transfer area and $T_{\mathrm{G}}$ is the average temperature of the flowing glycol/water solution and $T_{1}$ is the temperature of the inside surface of the pipe.

For forced convection, the heat transfer coefficient can be defined using Equation (4-4).

$$
h_{\mathrm{c}}=\frac{\mathrm{Nu}(k)}{D_{\mathrm{i}}}
$$

where $\mathrm{Nu}$ is the Nusselt Number for the flow, $k$ is the thermal conductivity of the flowing fluid, and $D_{\mathrm{i}}$ is the interior diameter of the pipe. 
The Nusselt number is a non-dimensional parameter based on the geometry and fluid properties. This value cannot be calculated simply based on fixed parameters, and as such, it was determined experimentally for the tested system, as discussed in detail in section 4.3.2. This value would need to be experimentally determined if the modelled system has significant deviations either in terms of fluid used or if geometry of the system is different than the pairing used within this study, and is an input to the model.

Combining Equations (4-3) and (4-4), while also calculating the heat transfer surface, the overall equation for the heat transfer through the forced convection from the flowing fluid to the pipe is shown in Equation (4-5).

$$
q_{\text {fluid_flow }}=\frac{\mathrm{Nu}(k) 2 \pi r_{\mathrm{i}} L_{\mathrm{n}}\left(T_{\mathrm{G}}-T_{1}\right)}{D_{\mathrm{i}}}
$$

where $r_{\mathrm{i}}$ is the interior radius of the pipe and $L_{\mathrm{n}}$ is the length of pipe of the given node in which the heat transfer is being calculated.

\subsubsection{Conduction through the Pipe Material}

Once the heat transfer from the fluid is determined, the next step is to determine the heat transfer through the actual pipe. As most pipes are metallic in nature, these will provide a minimal resistance to the heat transfer between the flowing fluid and the storage tank, but none the less should still be calculated and modelled in case a more insulating, or thick walled pipe is utilized. To model the transfer, the conduction for a cylindrical surface was utilized, and is shown in Equation (4-6).

$$
q_{\text {pipe }}=\frac{2 \pi k L_{\mathrm{n}}}{\ln \left(\frac{r_{2}}{r_{1}}\right)}\left(T_{2}-T_{1}\right)
$$

where $k$ is the thermal conductivity of the pipe material, $L_{n}$ is the length of pipe in the node being calculated, $T$ and $r$ are the temperature and radius at the given surfaces, with 
surface 1 is the interior of the pipe and surface 2 is the outer surface.

\subsubsection{Conduction through the Ice Buildup}

In addition to the conduction through the pipe material, the ice built up on the surface of the pipe will also create a resistance for the heat transfer between the flowing fluid and the storage tank. The heat transfer through the ice would be through conduction only, and as such, Equation (4-7) shows the heat transfer through the ice.

$$
q_{\text {ice }}=\frac{2 \pi k L_{\mathrm{n}}}{\ln \left(\frac{r_{3}}{r_{2}}\right)}\left(T_{3}-T_{2}\right)
$$

where all of the variables remain the same as the conduction through the pipe, and where surface 3 is the outside surface of the ice, while surface 2 is the outer surface of the submerged pipe, which, when ice has formed would be the interface between the pipe and the ice.

One thing to note is if the ice has not formed $r_{3}=r_{2}$, which would create a situation where there is a division by 0 , making the heat transfer undefined at this point. This is true when looking at only the heat transfer through the pipe, and would be accurate if there is no ice, but mathematically will be taken care of once the composite heat transfer through the whole system is taken in to consideration, removing this error when no ice has formed.

\subsubsection{Natural Convection between the Pipe/Ice Surface and Tank Fluid}

The final heat transfer calculation is the natural convection heat transfer from the surface of the pipe or ice, into the fluid of the tank. To calculate this, the base convection heat transfer equation is first utilized, as shown in Equation (4-8) with the specific parameters of the given situation. 


$$
q=h_{\mathrm{c}} A\left(T_{3}-T_{\mathrm{T}}\right)
$$

where surface 3 is the interface between the outside of the pipe/ice and the fluid in the tank, while position $T$ is the temperature of the fluid in the tank.

As the fluid in the tank is naturally at rest, with no movement unless active air conditioning is being provided, it is assumed that all heat transfer into the tank from the surface is via natural convection. Natural convection is based on the change in buoyancy of the fluid as the fluid closest to the surface either increases/decreases in temperature, changing the fluid density, and as a result, the movement of fluid around cooling surface. The convective heat transfer coefficient of a submerged pipes has been previously determined and available through literature, and is shown in Equation (4-9).

$$
h_{\mathrm{c}}=\frac{C(\mathrm{Ra})^{n} k}{D}
$$

where $\mathrm{Ra}$ is the Rayleigh number, which is a dimensional parameter defined in Equation (4-10),

$$
R a=\frac{g \beta\left(T_{3}-T_{\mathrm{T}}\right) D^{3}}{v \alpha}
$$

where $g$ is the acceleration due to gravity $\left(9.81 \mathrm{~m} / \mathrm{s}^{2}\right), \beta$ is the volumetric expansion of the tank fluid $(1 / \mathrm{K}), v$ is the kinematic viscosity of the tank fluid and $\alpha$ is the thermal diffusivity of the tank fluid, while $C$ and $n$ are constants, which depend on the Rayleigh Number and are defined in Table 4-4..

Table 4-4: Constant values for calculating natural convection around a cylinder

\begin{tabular}{c|cc}
\hline Rayleigh Number & $\mathrm{C}$ & $\mathrm{n}$ \\
\hline$<0.01$ & 0.675 & 0.148 \\
\hline $0.01-100$ & 1.02 & 0.148 \\
\hline $100-10,000$ & 0.85 & 0.188 \\
\hline$>10,000$ & 0.48 & 0.25 \\
\hline
\end{tabular}


Combining all of the pieces, the overall heat transfer as a result of the natural convection between the pipe/ice and the tank is given in Equation (4-11).

$$
q_{\text {Nat_conv }}=\frac{C(\mathrm{Ra})^{n} k 2 \pi r_{3} L_{\mathrm{n}}\left(T_{3}-T_{\mathrm{T}}\right)}{D}
$$

\subsubsection{Calculating the Total Heat Transfer}

Once the heat transfer for each individual mechanism was defined, the total heat transfer through the system has to be calculated. If the assumption is made that the system is operating at a quasi steady state, and the amount of energy stored within the pipe and the ice constant, the heat transfer through each component must be constant. As such, the following statement, as shown in Equation (4-12), can be made.

$$
q=q_{\text {Nat_conv }}=q_{\text {ice }}=q_{\text {pipe }}=q_{\text {fluid_flow }}
$$

Using this relationship, as well as the relationships between the temperatures at the common interfaces, a relationship for the total heat transfer from the flowing fluid into the storage tank, referred to as the charging heat transfer, can be calculated using Equation (4-13).

$$
=\frac{q_{\text {charging,n }}}{\frac{D}{2 \pi r_{1} L_{\mathrm{n}} N u\left(k_{\text {fluid }}\right)}+\frac{T_{\mathrm{G}}-T_{\mathrm{T}}}{2 \pi L_{\mathrm{n}} k_{\text {pipe }}}+\frac{\ln \left(\frac{r_{3}}{r_{2}}\right)}{2 \pi L_{\mathrm{n}} k_{\text {ice }}}+\frac{D}{2 \pi r_{3} L_{\mathrm{n}} C(R a)^{n} k_{\text {tank }}}}
$$

To simplify the length of this equation, all items on the bottom of the fraction will be denoted as $R_{\text {Total }}$. From this equation, we can also calculate the outlet temperature of the flowing fluid. As the total heat transfer into or out of the storage tank must equal the rate of change of the energy in the fluid as defined in Equation (4-14), 


$$
q=\dot{m} c_{\mathrm{p}}\left(T_{\text {out }}-T_{\text {in }}\right)
$$

where $\dot{m}$ is the mass flow rate of the flowing fluid, $c_{p}$ is the specific heat of the flowing fluid, $T_{\text {in }}$ is the temperature of the flowing fluid as it enters the node being modelled, while $T_{\text {out }}$ is the temperature at the outlet.

Combining Equation (4-13) and (4-14), the outlet temperature of the node can be defined using Equation (4-15).

$$
T_{\text {out }}=T_{\mathrm{T}}-\exp \left(\frac{-1}{\dot{m} c_{\mathrm{p}} R_{\text {Total }}}\right)\left(T_{\mathrm{T}}-T_{\mathrm{in}}\right)
$$

Finally the total heat transfer for a given pipe can be calculated through the sum of each of the nodes, using Equation (4-16).

$$
q_{\text {charging,pipe }}=\sum_{n=1}^{12} q_{\text {charging,n }}
$$

Using this value, the total rate at which the system is charging at can be calculated using Equation (4-17), where the heat transfer rate is multiplied by the total number of pipes, which is inputted as a parameter, based on the number of rows and columns of pipes.

$$
q_{\text {charging }}=q_{\text {charging,pipe }}(\text { columns })(\text { rows })
$$

This value represents the total amount of cooling potential stored at a given time step (or the amount of energy removed from the thermal storage system), and is the value utilized to determine net change in cooling potential of the system for a given time step.

\subsubsection{Modelling the Pipe/Ice Surface Temperature}

Equations (4-13) and (4-15) provide the method for calculating the total heat transfer and outlet temperature, however, to calculate the Rayleigh number, the surface 
temperature of the pipe or of the outside surface of the ice must be known, as this drives the natural convection. This surface temperature can only be determined after the overall heat transfer is known, at with point all of the interface temperatures can be found. As such, an initial assumption for that surface temperature must be made for each time step. Within the model, this value is always set as $0^{\circ} \mathrm{C}$. Once the total heat transfer is determined, this surface temperature is then calculated, and the entire heat transfer rate is recalculated. Within the model, 15 iterations are completed to ensure an accurate surface temperature and therefore heat transfer rate can be determined. With a range of test conditions, a convergence within $0.1 \%$ of the total heat transfer rate was always achieved within 5 iterations, however, 15 iterations were chosen to ensure that no matter the situation, the true surface temperature will be determined.

\subsubsection{Modelling Tank Temperature and Ice Formation}

Once the heat transfer into the storage tank was calculated, the overall energy balance on the storage tank had to be calculated. In addition to the heat transfer out of the tank through the charging pipes, decreasing its temperature, heat transfer into the tank occurs from two sources. The first is through the walls of the storage tank, while the second is through the use of the stored cooling potential to meet the air conditioning load of the house. These two values must be calculated, and then used in conjunction with the cooling potential added to the system through the submerged pipes to determine the net change in stored cooling potential for a given time step.

First, the energy transfer across the tank wall had to be calculated. The area of the tank wall, the thermal conductivity of the tank wall, the tank wall thickness, and the temperature of the air surrounding the tank were all inputted into the simulations as 
parameters, and all were considered constant throughout the length of any simulation. The actual heat transfer was calculated using Equation (4-18),

$$
q_{\text {loss }}=A_{\text {Tank }_{\text {wall }}}\left(T_{\text {Tank }}-T_{\text {surrounding }}\right) \frac{k_{\text {Tank_wall }}}{t_{\text {Tank_wall }}}
$$

where $A$ is the area, $k$ is the thermal conductivity, $t$ is the thickness, and $T$ is the temperature.

In this case, all values are constant throughout the length of the simulation, with the exception of the tank temperature, which is dependent on the temperature of the fluid within the tank at any given time step.

The second component which the change in energy required is the amount of cooling delivered to the building cooling coil. Based on the assumption that the tank fluid is a constant temperature through the entire system, it is further assumed that the tank temperature is also the temperature delivered to the cooling coil. The total amount of cooling delivered to the cooling coil, and consequently the amount of cooling potential removed from the cold thermal storage is calculated using Equation (4-19),

$$
q_{\mathrm{AC}}=\dot{m}_{\mathrm{water}} c_{\mathrm{p}}\left(T_{\text {Tank }}-T_{\text {Return }}\right)
$$

where $\dot{m}$ is the mass flow rate in $\mathrm{kg} / \mathrm{s}, c_{\mathrm{p}}$ is the specific heat of the tank fluid, and $T$ is the temperature at the given locations.

Using these energy transfer rates, the net change in energy can be calculated for a given time step for the model. The equations were set up so that the energy transfer is positive for $q_{\text {charging }}$ when cooling potential is being added to the system (energy being removed), while $q_{\text {loss }}$ and $q_{\mathrm{AC}}$ are negative as cooling potential is being removed from the system (energy being added). As such, the total change in energy is defined using Equation (4-20). 


$$
q_{\text {net }}=q_{\text {charging }}+q_{\text {loss }}+q_{\mathrm{AC}}
$$

This net change in energy transfer rate was then converted into the total amount of energy transferred during a given time step, by multiplying $q_{\text {net }}$ by the timestep in seconds.

If the net energy for the given time step is greater than zero, then the system is charging, and additional cooling potential is being added into the system. If the net energy is less than zero, the stored cooling potential is being depleted. If the net change is positive and the system is charging, the first step is to determine the whether the energy will be stored as through sensible or latent storage. An underlying assumption that has been made within this model is that all energy is stored sensibly until all of the water within the tank has reached $0^{\circ} \mathrm{C}$, and then all additional energy is stored through the latent change of the water to ice. As such, a new tank temperature is first calculated using the amount of energy stored, using the change in energy, tank volume and the fundamental fluid properties of the storage water. If that temperature is determined to be greater than $0^{\circ} \mathrm{C}$, then all energy is stored through the sensible change in the tank temperature, and the tank temperature is set as the new value and the system storage is set for that time step. If the tank temperature is found to be less than $0^{\circ} \mathrm{C}$, then a certain amount of energy must be stored as latent energy. In this case, the total amount of additional energy that needs to be stored after meeting the $\mathrm{AC}$ and loss demands, as well as bringing the tank temperature to $0^{\circ} \mathrm{C}$ is calculated. As the total energy transferred for each node is not equal (nodes near the pipe inlet have colder fluid in them, and therefore a greater temperature difference), this energy is proportioned per node, based on the ratio of the energy transfer at that node compare to the total pipe heat transfer, using Equation (4-21). 


$$
q_{\text {latent, } \mathrm{n}}=\frac{q_{\text {charging, } \mathrm{n}}}{q_{\text {charging,pipe }}} q_{\text {latent,pipe }}
$$

Once $q_{\text {latent,n }}$ is determined of each node, the mass of the ice formation around each node was determined using the heat of formation of water. Based on the mass, using the density of ice and the volume, the thickness of the ice at each node was determined. It was important to solve this proportionally for each node, as ice formation does not form equally along the length of the pipe, and if the pipe was assumed as a single node, a uniform thickness would be found which does not accurately represent how the ice would actually form. This equation is based on the underlying assumption that the amount of energy each node provides to meet the AC loads and loss within the tank is taken proportionately from each node, based on its charging rate compared to the total charging rate.

If the net energy had been found as less than zero, the system would be overall reducing the amount of cooling potential stored. It was assumed that the net decrease was absorbed equally across all nodes within the systems. Once the energy transfer rate is determined for each node, the increase in energy is utilized to first melt the ice that is formed on the submerged pipe. Once the ice is melted on a given node, its allotment of the energy increase in the system is used to increase the sensible temperature within the tank, increasing the overall tank temperature.

At the end of each time step, once the final mass of ice and tank temperatures were determined, the overall amount of cooling potential being stored was calculated. This value was the sum of the energy stored through the sensible change in the tank fluid and the latent energy stored through the phase change of the water to ice. Equation (4-22) takes into account both of these and provides the total energy stored, which is used as the control 
variable for this system.

$$
E_{\text {stored }}=V \rho c_{\mathrm{p}}\left(T_{\mathrm{Ref}}-T_{\text {Tank }}\right)+m_{\text {ice }} h_{\mathrm{fg}}
$$

where $V$ is the volume of fluid in the tank, $T_{\text {Ref }}$ is an input parameter and is the reference temperature in which the sensible storage is calculated relative to (typically the warmest temperature cooling can still be realized for a given application), $m_{\text {ice }}$ is the mass of ice that has formed within the tank and $h_{\mathrm{fg}}$ is the heat of formation of ice.

This series of equations formed the basis for a code first written in Matlab to test the functionality of code, then converted to a Fortran code in the TRNSYS format, where the parameters, inputs and outputs for the TRNSYS component were also defined. The final code for the developed ice storage model in TRNSYS is provided in Appendix E.

\subsubsection{Model Experimental Validation}

Once the model was developed, it needed to be validated against experimental results to confirm the methodology developed can accurately model the performance of the system. A number of experimental test runs were conducted, where the inlet conditions (flow and temperature) entering the system were recorded and used as the input for the model. The experimental and modelled heat transfer and total energy storage were across the length of the test were then compared to determine the model's accuracy. The 
parameters for the experimental test set-up were determined and inputted into the model as outlined in Table 4-5.

Table 4-5: Experimental parameters of the small-scale ice storage set-up

\begin{tabular}{c|c}
\hline Parameter & Value \\
\hline Water Volume & $21.8 \mathrm{~L}$ \\
\hline Number of Pipe(s) & 1 \\
\hline Length of Pipe & $1.3 \mathrm{~m}$ \\
\hline Pipe Inner Diameter & $0.008 \mathrm{~m}$ \\
\hline Pipe Thickness & $0.00076 \mathrm{~m}$ \\
\hline $\begin{array}{c}\text { Thermal Conductivity } \\
\text { of the Pipe Material }\end{array}$ & $401 \mathrm{~W} / \mathrm{K} \cdot \mathrm{m}$ \\
\hline Tank Surface Area & $0.7096 \mathrm{~m}^{2}$ \\
\hline $\begin{array}{c}\text { Thermal Conductivity } \\
\text { of Tank Wall }\end{array}$ & $0.033 \mathrm{~W} / \mathrm{K} \cdot \mathrm{m}$ \\
\hline
\end{tabular}

The final parameter required is the Nusselt number $(\mathrm{Nu})$, which is a nondimensional parameter that relates the convective to conductive heat transfer that occurs at the heat transfer boundary of a moving fluid. This parameter is dependent on the fluid properties, the flow conditions and the geometry of the fluid passing through. In literature, a $\mathrm{Nu}$ value of 4-4.5 is prescribed [77], however no value was found for a glycol/water solution at low temperatures flowing through the pipe. As such, the first experiment was used to determine the $\mathrm{Nu}$ that should be used for the system. A parametric study was conducted with the $\mathrm{Nu}$ number ranging from 6-9 in increments of 1 . The graph in Figure 4-22 show the experimental heat transfer rate compared to the modelled heat transfer rate with each of the different $\mathrm{Nu}$ values, while Table 4-6 shows the sum of the absolute differences between the experimental and modelled heat transfer rates, with $\mathrm{Nu}$ values of 6.5 and 7.5 added for comparison. 


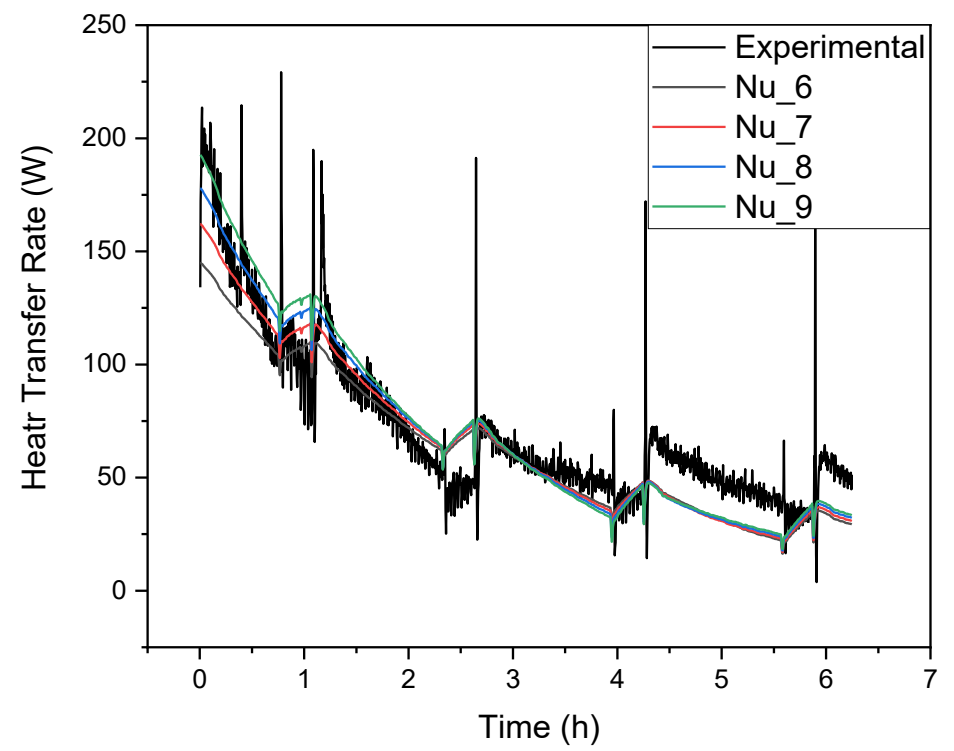

Figure 4-22: Comparison of different Nusselt numbers to the experimental values

Table 4-6: Results for the absolute differences for each Nusselt number modelled

\begin{tabular}{c|c}
\hline Nusselt Number & $\begin{array}{c}\text { Sum of } \\
\text { Absolute } \\
\text { Difference }\end{array}$ \\
\hline 6 & 19954 \\
\hline 6.5 & 19036 \\
\hline 7 & 18574 \\
\hline 7.5 & 18481 \\
\hline 8 & 18801 \\
\hline 9 & 20169 \\
\hline
\end{tabular}

Based on these results, a value of 7.5 was chosen as the value for the Nusselt number going forward. It was also noted that the Nusselt number had a much greater impact on the results, when the heat transfer rates were highest and the temperature difference between the storage tank and the flowing heat transfer fluid. This is because during these periods, the heat transfer coefficient from the flowing fluid to the pipe is much closer to the heat transfer coefficient of the natural convection in the tank. As such, the forced convection has a much greater impact on the overall heat transfer through the system. When the tank temperature is much closer to the flowing fluid temperature, the natural convection 
coefficient is much lower than the forced convection coefficient, which includes the Nusselt number. As such, the natural convection within the system is the limiting factor in the overall heat transfer of the system. When these conditions are present, the Nusselt number has a limited impact on the overall heat transfer taking place.

Once the Nusselt number was experimentally determined, the results obtained using the developed modelled were compared to experimentally obtained date. The inlet flow rate and temperature of the heat transfer fluid within the experiment were recorded and a text file was created with this data. This file was then used as the input to the ice storage component, and the outlet temperature, storage fluid temperature, and the amount of energy stored within the system were compared between the modelled system and the experimental systems. Two experimental runs were used to validate the model of the ice storage, where the starting tank temperature and the flow rate through the pipe varied for the two tests. The comparison between outlet temperatures for the two tests are shown in Figure 4-24, the comparison between heat transfer rates is shown in Figure 4-25, while the total amount of cooling stored is shown in Figure 4-26. 

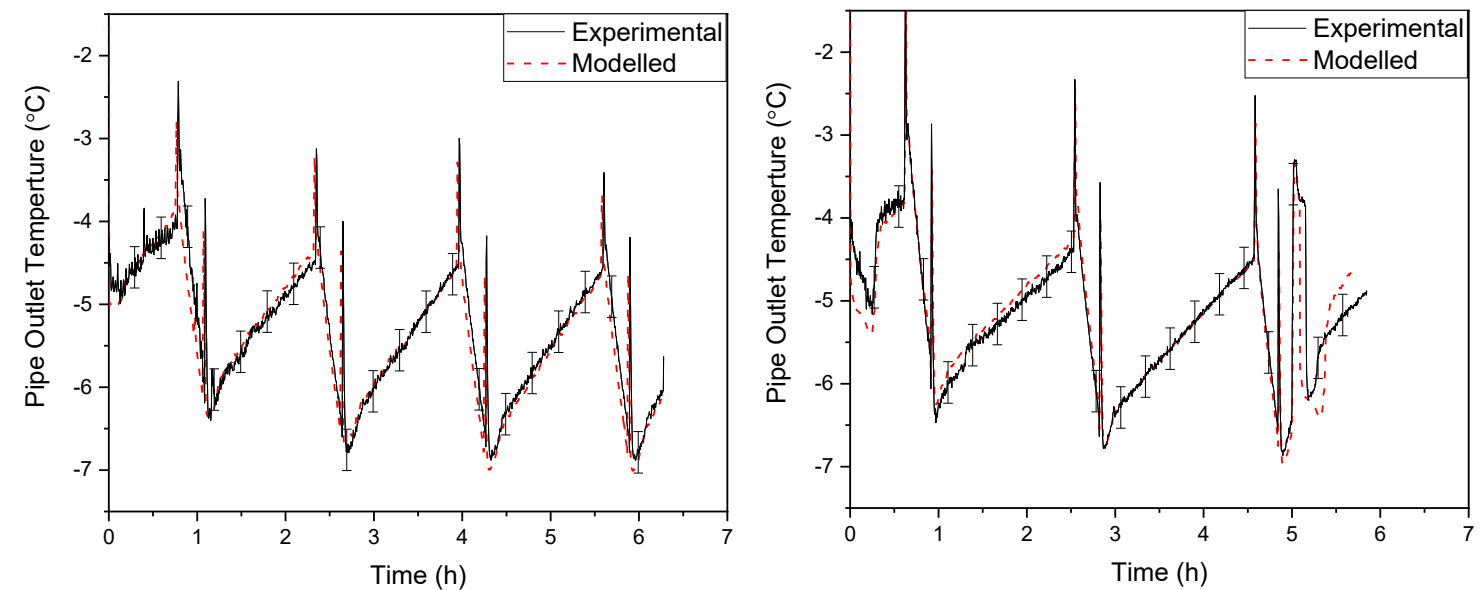

Figure 4-23: Comparison of pipe outlet temperature for two experimental runs
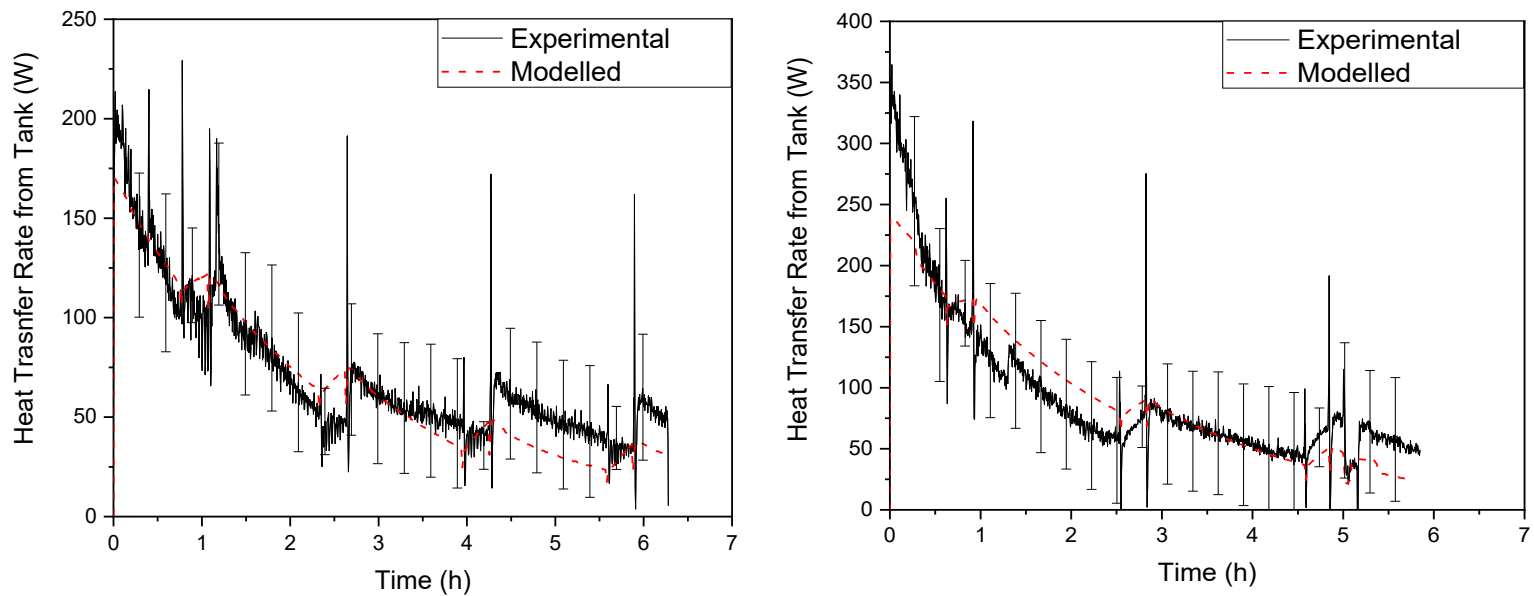

Figure 4-24: Comparison of heat transfer rates for two experimental runs
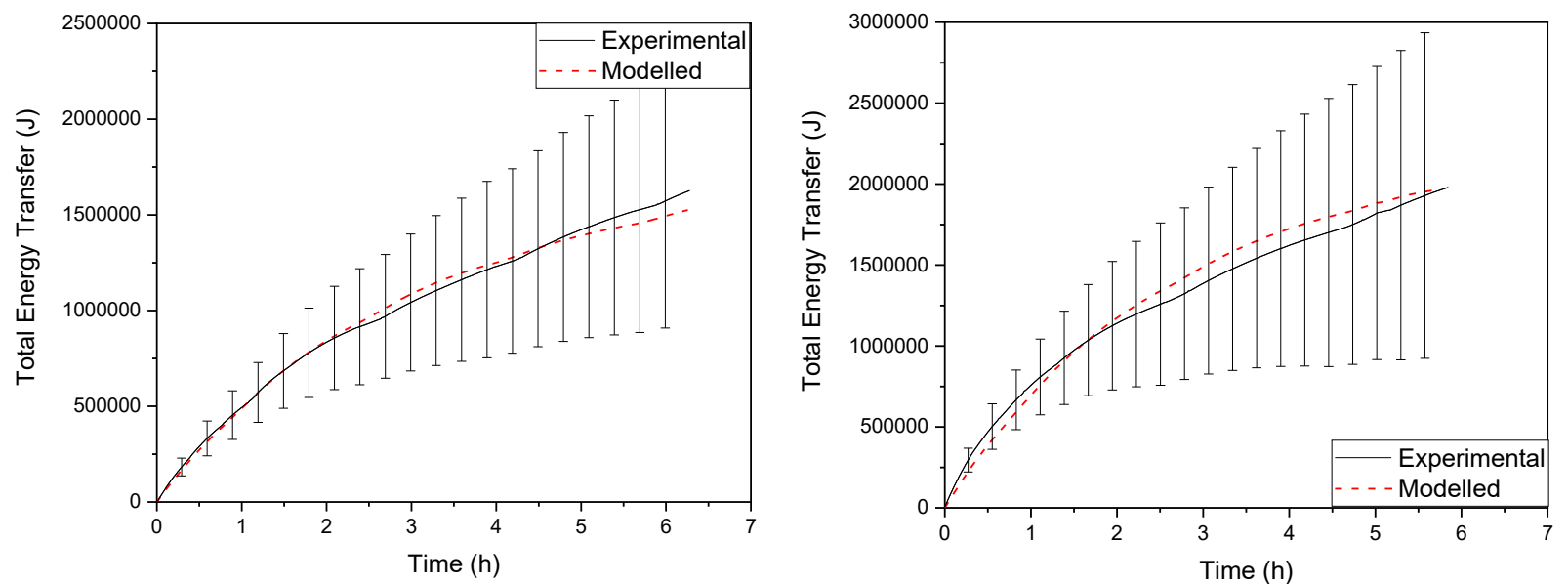

Figure 4-25: Comparison of total energy transfer for two experimental runs 
Based on these results, a number of conclusions can be drawn. The first is that in all cases, there is a good agreement between the experimental and modelled values, and in all cases, the modelled value is well within the range of the experimental uncertainty. As such, it can be said that the model accurately predicts the amount of heat transfer that occurs within the system. The second conclusion that can be drawn is that the experimental uncertainties within the system are quite large and are driven by the experimental uncertainty in the temperature readings. These uncertainties are even greater in the second test, where the flow rate was higher, which consequently caused the temperature difference across the length of the pipe to be smaller. As the uncertainty on the temperature measurement is a constant value, this uncertainty has a larger impact on the overall uncertainty on the heat transfer rate. To further validate the developed model, sensors with a much higher accuracy would be required. These measurements were conducted using thermocouples which have lower accuracies when compared to other measurement devices. Based on the small change in temperature across the pipe, sensors with a combined uncertainty on the change in temperature of $\pm 0.1^{\circ} \mathrm{C}$, which equates to approximately $5 \%$ of the change in temperature across the pipe, should be utilized to improve the experimental results. To meet these specifications, high accuracy RTDs would need to be utilized at both the inlet and outlet of the system, as discussed in Chapter 3.

In addition to the heat transfer rates, the tank temperature, ice accumulation and total energy stored within the system were compared between the experimental and modelled values. The modelled results were compared to the experimental results for the first test previously examined, and the results for the tank temperature are shown in Figure 4-26, ice accumulation in Figure 4-27, and the total energy stored in the system in 
Figure 4-28. As the experimental ice growth was only measured at discrete points, the ice accumulation and stored thermal energy is only shown at the points measurements were taken, while the modelled values are continuously reported.

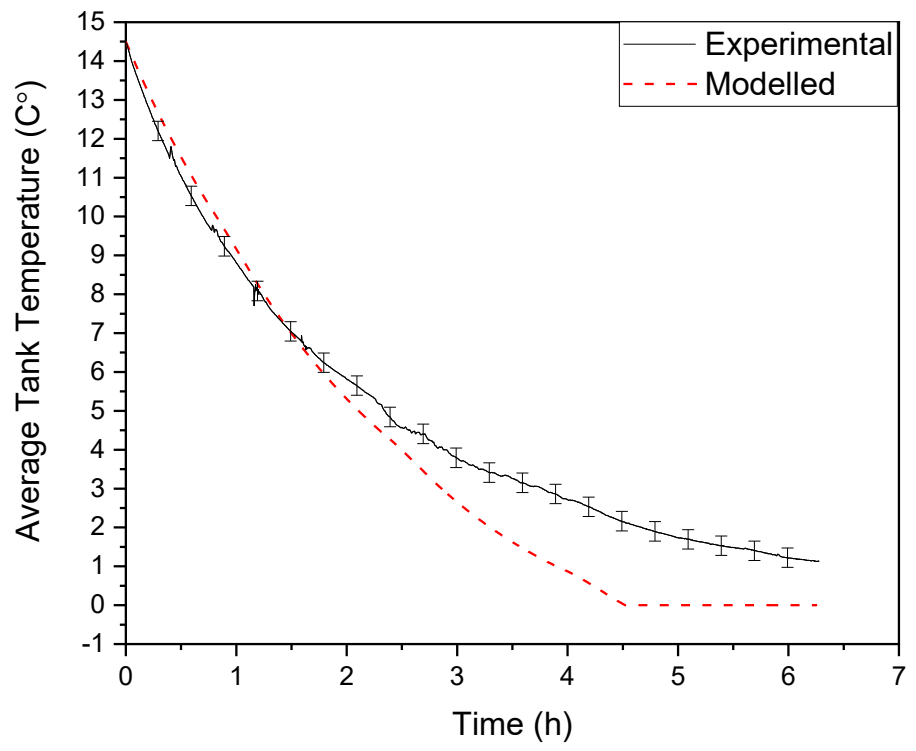

Figure 4-26: Comparison between modelled and experimental average tank temperature

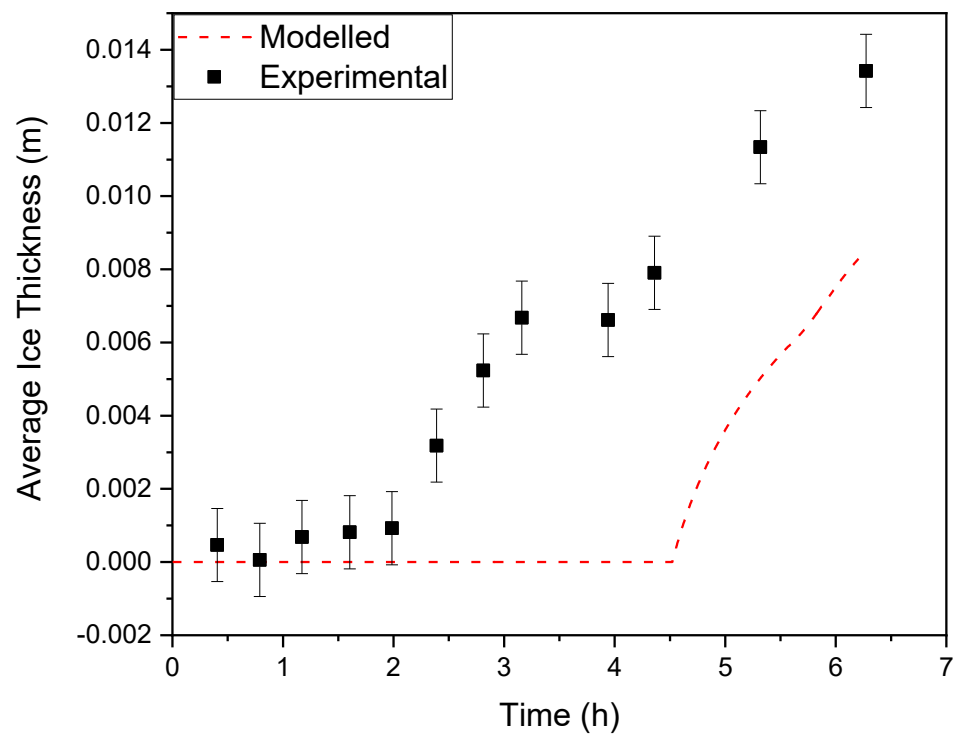

Figure 4-27: Comparison between modelled and experimental ice thickness 


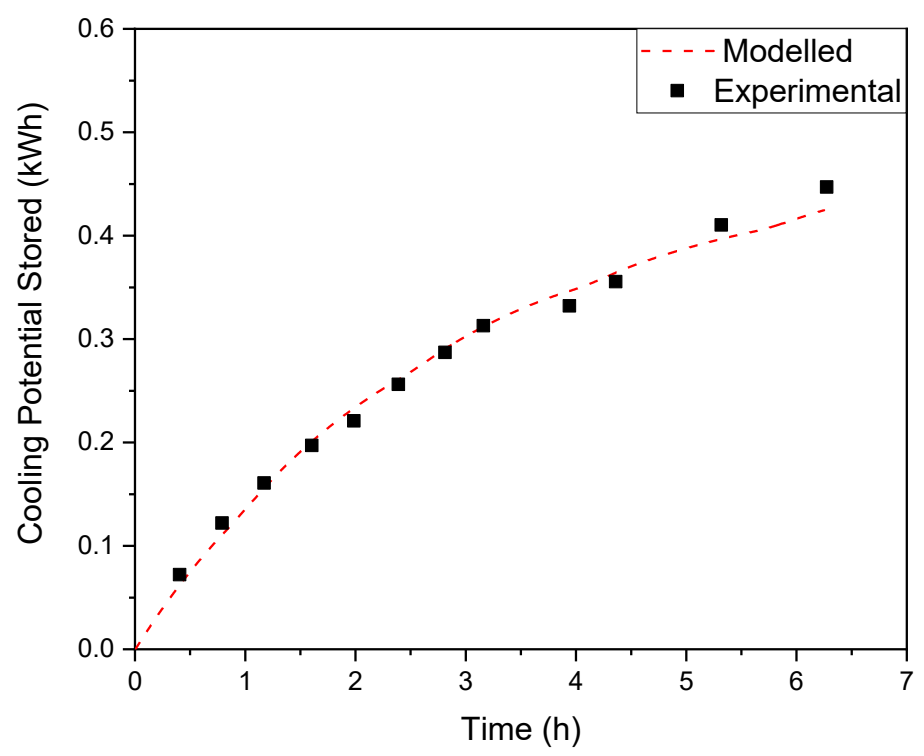

Figure 4-28:Comparison between modelled and experimental stored cooling potential

Based on these results, it can be seen that the model, when compared to the experimental set-up, accurately predicts and models the total amount of cooling potential that is being stored. Where the model lacks accuracy is in predicting the exact breakdown of sensible storage (through the storage tank temperature) and the latent storage through the transformation of the water to ice. This is caused by a combination of the assumptions made within the model and the limitations of the experimental set-up. In the model, it was assumed that the entire tank got to $0^{\circ} \mathrm{C}$ before any ice began to form, and then all cooling potential stored was in the formation of ice. In the small-scale test set-up, since there was no discharge capability, there was no movement in the storage water. This caused a temperature gradient to form within the tank, where the fluid closer to the pipe was much colder than that on the outside edge of the tank, far away from the pipe. This resulted in the water at the surface of the pipe in the experimental system to reach the freezing point earlier in the test period than the model predicted, and as such, ice started to form much earlier. As such, a greater percentage of the total cooling stored was in the latent form in 
the experiment compared to what was modelled. In an actual system, where there would be a semi-regular draw of cooling from the system, which would require the movement of the storage fluid, it is anticipated that the fluid would be much better mixed than in the experimental set-up. If this were the case, the assumption of constant temperature within the tank, and reaching $0^{\circ} \mathrm{C}$ before ice formation would be closer to reality, and the difference in the percentage between latent and sensible storage would be much lower. Based on this fact, it was determined that the model adequately represents the storage capabilities of an ice storage and will be used to determine the annual performance of the integrated system. 


\section{Chapter 5: Whole System and Building Modelling Methodology}

Once the individual components were modelled, the next step in developing a complete system which could be used to offset peak loads is to determine interaction between components and determine the required sizing of the systems. This chapter first examines the impact of flow rate between the chiller and the hot and cold thermal storage systems to determine the impact of flow on performance. The required configuration and sizing for the ice storage was then completed, to determine the piping layout and geometric constraints of the system when the validated model is brought into an actual application and finally, the interaction between the heat pump or chiller and the thermal storage systems are modelled and the optimal configurations determined.

\subsubsection{Charge Efficiency of the Sensible Thermal Storage}

Once the model of the system was complete, the first step was to look only at the interaction between the different heat pumps being tested and the thermal storage systems. The main drawback to using a thermal storage system with a heat pump is that the inlet temperature from the cold storage to the evaporator is lower, while the inlet temperature from the hot thermal storage to the condenser is higher, when compared to using the heat pump in a standard configuration with direct heating/cooling. These increase/decrease in temperatures can cause a significant degradation in performance of the heat pump. As part of this study, one research question was if the use of a stratified storage tank, which would use lower flow rates through the heat pump to prevent mixing, allowing a higher/lower temperature for a longer period of time would improve overall performance. The tradeoff for these more advantageous temperatures is lower flow rates, which reduce the heat transfer potential through the system. As such, a modelling study was utilized to determine 
the optimal flow rates for the complete system and determine whether the use of a stratified storage tank could improve system performance.

This study was conducted using the same size storage capacity as the experimental set-up, with the glycol/water solution as the cold thermal storage medium. The cold tank had a volume of $270 \mathrm{~L}$, while the hot tank had a volume of $300 \mathrm{~L}$. In each test, the tanks were set at $20^{\circ} \mathrm{C}$ and the heat pump was set to run continuously for two hours, while the medium temperature chiller was set to operate for 1 hour based on its increased cooling capacity. This study was done in two parts, first holding the hot, condenser side at a constant temperature and flow, and charging the cold thermal storage at a constant flow, and then holding the cold, evaporator side constant, and charging the hot thermal storage at a constant temperature.

\subsubsection{Impact of Flow on Cold Thermal Storage Charging}

Before modelling as the complete system, the cold side was first examined with a hot side inlet temperature of $25^{\circ} \mathrm{C}$ and a flow rate for $480 \mathrm{~kg} / \mathrm{hr}$, and the cold side flow rate was varied from $200 \mathrm{~kg} / \mathrm{hr}$ to $500 \mathrm{~kg} / \mathrm{hr}$ in $50 \mathrm{~kg} / \mathrm{hr}$ increments. The results for the standard heat pump are shown in Figure 5-1. This was repeated for the medium temperature chiller, with a fixed condenser temperature of $25^{\circ} \mathrm{C}$ and a flow of $900 \mathrm{~kg} / \mathrm{hr}$, and cold side flow rates of $300 \mathrm{~kg} / \mathrm{hr}$ to $900 \mathrm{~kg} / \mathrm{hr}$ on $100 \mathrm{~kg} / \mathrm{hr}$ increments, with the results shown in Figure 5-2. The total charge time for each test is different (length of test), based on the difference in capacity of the heat pump and medium temperature chiller. 


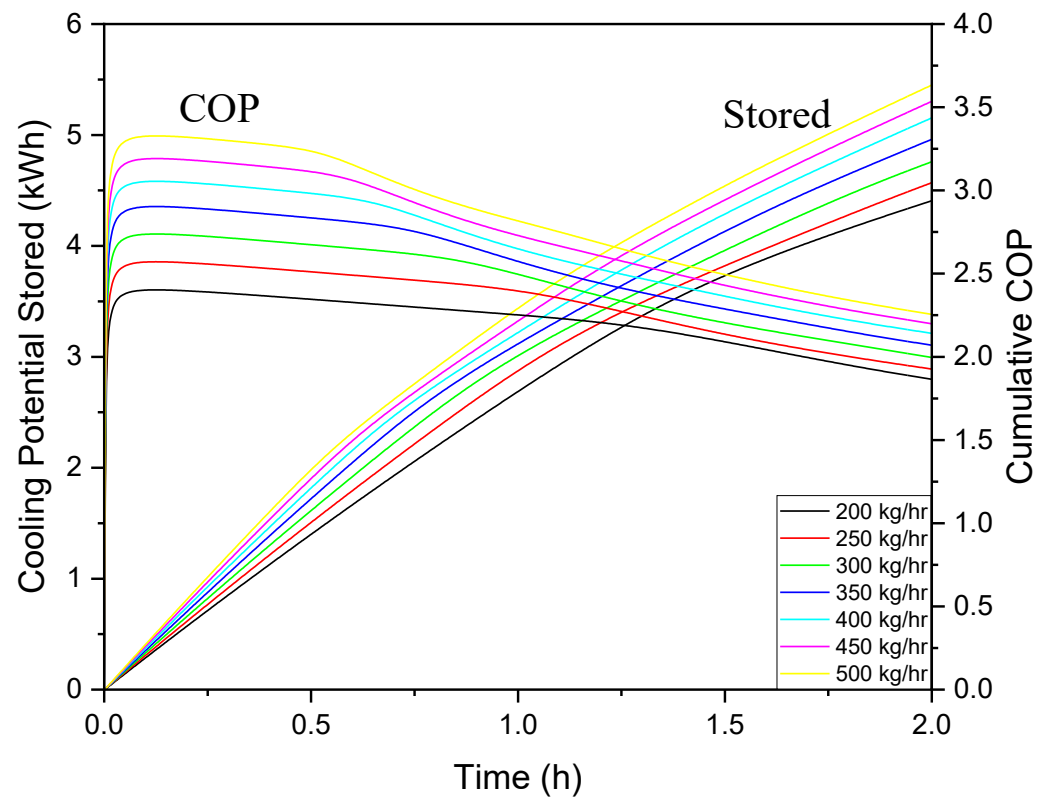

Figure 5-1: Comparison of cold flows for the standard heat pump

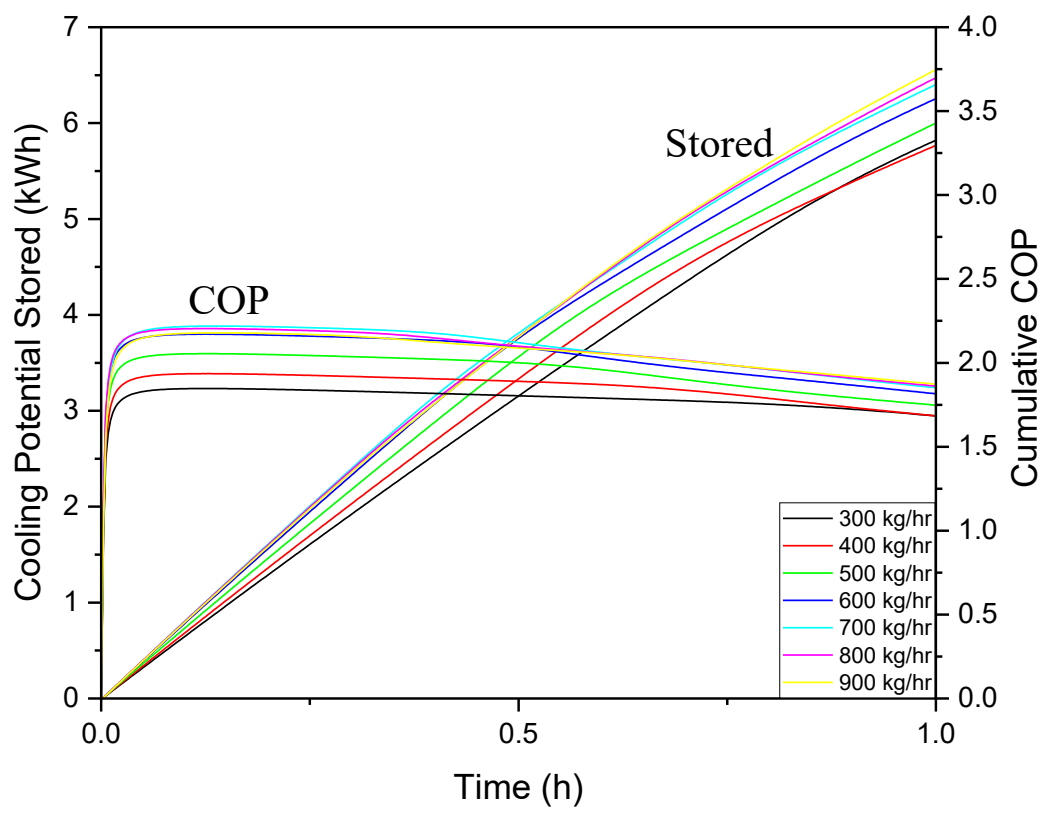

Figure 5-2: Comparison of cold flows for the medium temperature chiller

From these results, a number of conclusions were determined. The first, and most important is the heat pump performance obtained using a stratified thermal storage tank is lower than that using a well-mixed, higher flow rate configuration. The average inlet temperatures over the length of the tests, as shown in Table 5-1 for the standard heat pump 
and Table 5-2 for the medium temperature chiller, increased with the decreased flow rate, however the total cooling potential stored, as well as the overall COP to charge the tank decreased. As such, it was determined that, when directly coupling a heat pump/chiller to a cold thermal storage system, higher flow rates are more desirable for the overall performance of the system.

Table 5-1: Results at the end of each test with varying flow rates - Heat Pump

\begin{tabular}{l|llll}
\hline $\begin{array}{l}\text { Flow } \\
\text { Rate } \\
(\mathrm{kg} / \mathrm{hr})\end{array}$ & $\begin{array}{l}\text { Average } \\
\text { Evaporator } \\
\text { Temperature }\left({ }^{\circ} \mathrm{C}\right)\end{array}$ & $\begin{array}{l}\text { Stored Cooling } \\
\text { Potential } \\
(\mathrm{kWh})\end{array}$ & $\begin{array}{l}\text { Electrical } \\
\text { Input } \\
(\mathrm{kWh})\end{array}$ & $\begin{array}{l}\text { Tank } \\
\text { Charging } \\
\text { COP }\end{array}$ \\
\hline 200 & 16.30 & 4.41 & 2.36 & 1.87 \\
250 & 15.05 & 4.57 & 2.37 & 1.93 \\
300 & 14.05 & 4.75 & 2.38 & 2.00 \\
350 & 13.07 & 4.96 & 2.40 & 2.07 \\
400 & 12.33 & 5.15 & 2.41 & 2.14 \\
450 & 11.67 & 5.30 & 2.41 & 2.20 \\
500 & 11.03 & 5.44 & 2.42 & 2.25 \\
\hline
\end{tabular}

Table 5-2: Results at the end of each test with varying flow rates - Chiller

\begin{tabular}{l|llll}
\hline $\begin{array}{l}\text { Flow } \\
\text { Rate } \\
(\mathrm{kg} / \mathrm{hr})\end{array}$ & $\begin{array}{l}\text { Average } \\
\text { Evaporator } \\
\text { Temperature }\left({ }^{\circ} \mathrm{C}\right)\end{array}$ & $\begin{array}{l}\text { Stored Cooling } \\
\text { Potential } \\
(\mathrm{kWh})\end{array}$ & $\begin{array}{l}\text { Electrical } \\
\text { Input } \\
(\mathrm{kWh})\end{array}$ & $\begin{array}{l}\text { Tank } \\
\text { Charging } \\
\text { COP }\end{array}$ \\
\hline 300 & 18.14 & 5.82 & 3.45 & 1.68 \\
400 & 15.07 & 5.76 & 3.42 & 1.68 \\
500 & 13.54 & 6.00 & 3.43 & 1.74 \\
600 & 12.25 & 6.26 & 3.44 & 1.82 \\
700 & 11.18 & 6.40 & 3.46 & 1.85 \\
800 & 10.68 & 6.47 & 3.47 & 1.86 \\
900 & 10.22 & 6.56 & 3.50 & 1.87 \\
\hline
\end{tabular}

In addition to the conclusions made in terms of the performance of the systems in relationship to the flows through the evaporators, a comparison can be made between the two systems as well. It is noted that in the initial charging period, where temperatures and flows are high, the standard heat pump is able to charge with a much higher COP, in excess 
of 3 , while the medium temperature chiller hovers around a $\mathrm{COP}$ of 2 . This shows that for straight cooling of a building, the standard heat pump offers much better performance, as well as if only a small amount cooling potential needs to be stored. The benefits of the medium temperature chiller can be seen as the total stored quantity increase and, consequently the evaporator temperature decreases. The standard heat pump has a very low $\mathrm{COP}$, in some cases an instantaneous COP of less than 1 when temperature approach and go below $0^{\circ} \mathrm{C}$ and the heat pump stops functioning between $0^{\circ} \mathrm{C}$ and $-5^{\circ} \mathrm{C}$ depending on the flow conditions. When the medium temperature chiller is utilized, COPs between 1.5 and 2 are observed as the inlet evaporator temperatures reach $0^{\circ} \mathrm{C}$ and continue with limited degradation down to approximately $-10^{\circ} \mathrm{C}$ inlet temperatures. Based on these items, the medium temperature chiller can store between an additional $50 \%$ and $100 \%$ of cooling potential compared to the standard heat pump, reducing the required size of the thermal storage system. Additionally, the medium temperature chiller should be able to provide the required cooling capacity and COPs to meet the requirements of the proposed ice storage system, which is modelled as a system in Section 5.2.

\subsubsection{Impact of Flow on Hot Thermal Storage Charging}

Once the impact of the cold side flow rate on the overall system performance was determined, a second set of simulations were run for the impact of the hot side flow rate between the thermal storage tank and the condenser. In this case, a $300 \mathrm{~L}$ tank containing pure water was used as the thermal storage and cold side was kept constant at $15^{\circ} \mathrm{C}$ inlet temperature to the evaporator and at a flow rate of $400 \mathrm{~kg} / \mathrm{hr}$ for the standard heat pump and $800 \mathrm{~kg} / \mathrm{hr}$ for the medium temperature chiller. The same test conditions were utilized, with the standard heat pump running for 2 hours, while the medium temperature chiller ran 
for 1 . The result for the heat pump, with the hot side flow rate varying from $200 \mathrm{~kg} / \mathrm{hr}$ to $500 \mathrm{~kg} / \mathrm{hr}$ in $100 \mathrm{~kg} / \mathrm{hr}$ increments are shown in Figure 5-3, while the results for the medium temperature chiller, with flows varying from $400 \mathrm{~kg} / \mathrm{hr}$ to $1200 \mathrm{~kg} / \mathrm{hr}$ in $200 \mathrm{~kg} / \mathrm{hr}$ increments are shown in Figure 5-4. 


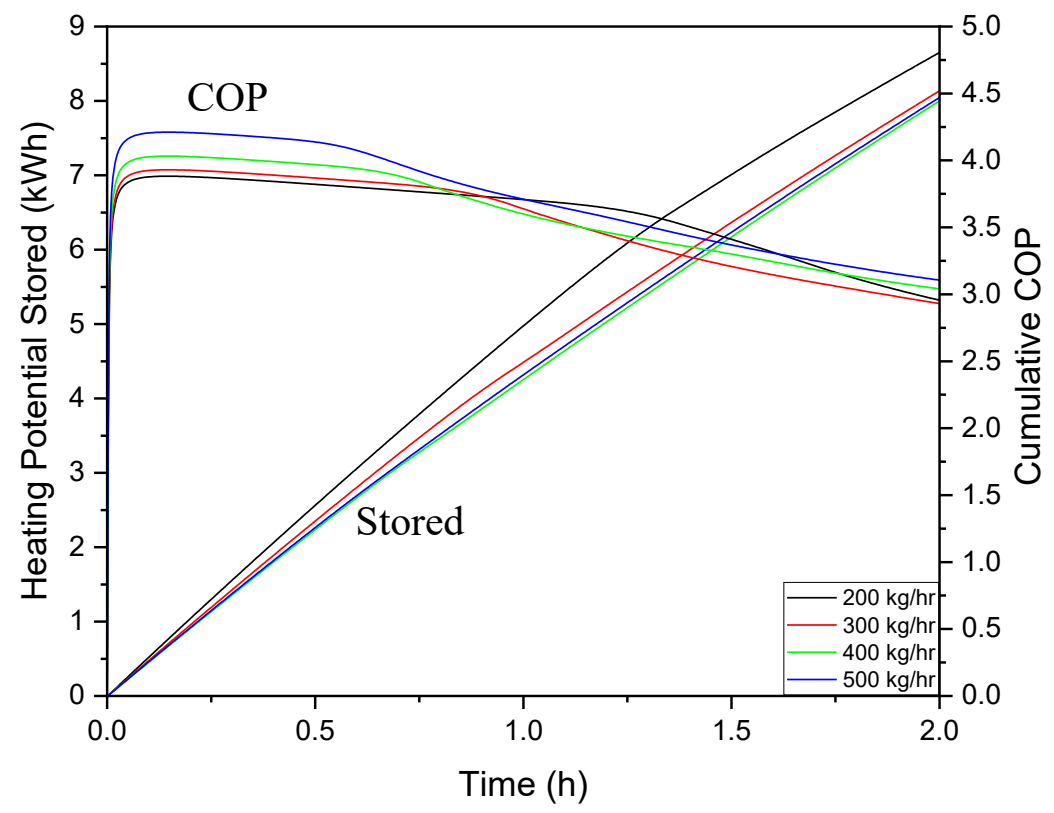

Figure 5-3: Comparison of hot flows for the heat pump

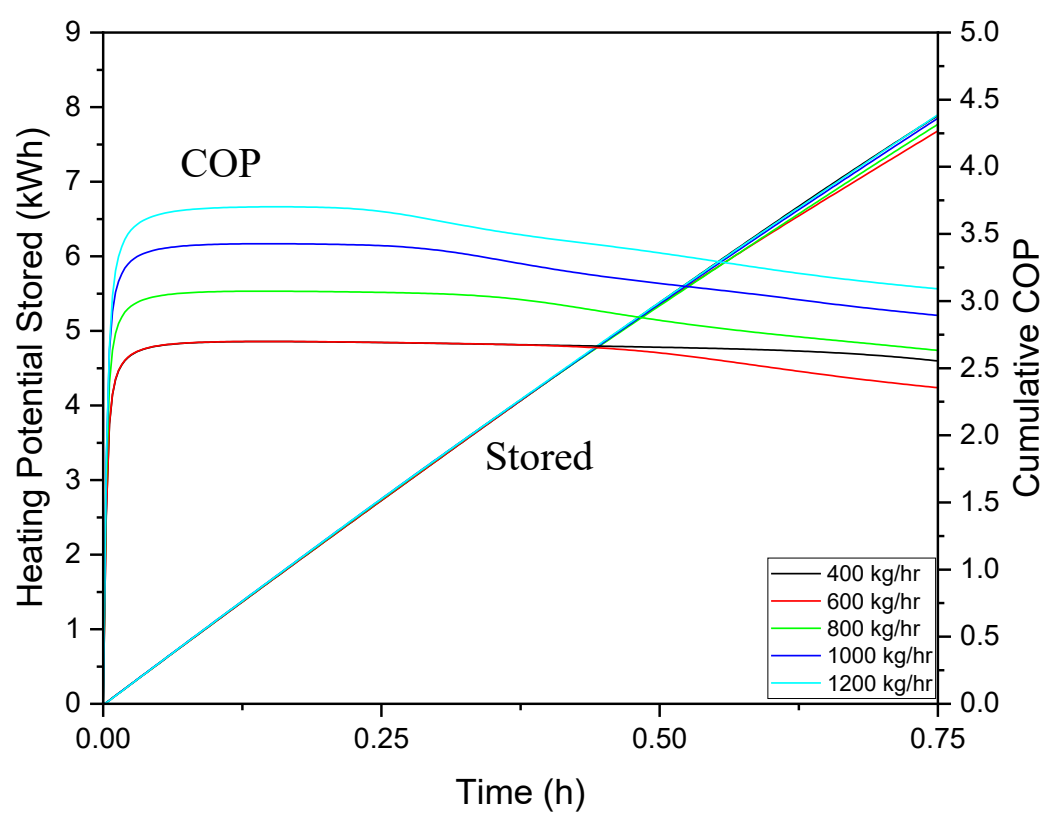

Figure 5-4: Comparison of hot flows for the medium temperature chiller

These results showed that at lower flow rates, the lower the flow rate, the better the overall performance of the system. This is because the amount of energy required to compress the refrigerant is directly related to the temperature in the condenser, so keeping 
the lower inlet temperatures for long periods of time improves performance. This trend continues until a tipping point, at which the increased flow, and consequently the increased heat transfer capability begins to improve performance, and in the end, flow rates on the higher end of what the standard heat pump and medium temperature chiller run have the overall, best COPs when compared to all modelled flow rates. Another item to note from these results is that the standard chiller starts with a very high COP (in excess of 4) when the condenser temperatures are in their lower range (less than $30^{\circ} \mathrm{C}$ ) and then drops significantly and quickly, ending with a total cumulative COP which ends up at approximately 3 . The medium temperature chiller starts with a much lower COP at the lower condenser temperatures (approximately 3.5), but does not see the drastic drop off as the condenser temperature increases, and the final result is very similar to that of the standard heat pump. This shows that the same charging profile is available with both units, while the medium temperature chiller can also reach those low temperatures required on the cold thermal storage side, be it sensible or latent storage. The total, final results for each of the flow rates are shown in Table 5-3 and Table 5-4.

Table 5-3: Results at the end of each modelled hot flow rates with the heat pump

\begin{tabular}{l|llll}
\hline $\begin{array}{l}\text { Flow } \\
\text { Rate } \\
(\mathrm{kg} / \mathrm{hr})\end{array}$ & $\begin{array}{l}\text { Average } \\
\text { Evaporator Inlet } \\
\text { Temperature }\left({ }^{\circ} \mathrm{C}\right)\end{array}$ & $\begin{array}{l}\text { Stored Heating } \\
\text { Potential } \\
(\mathrm{kWh})\end{array}$ & $\begin{array}{l}\text { Electrical } \\
\text { Input } \\
(\mathrm{kWh})\end{array}$ & $\begin{array}{l}\text { Tank } \\
\text { Charging } \\
\mathrm{COP}\end{array}$ \\
\hline 200 & 24.55 & 8.65 & 2.93 & 2.96 \\
300 & 26.32 & 8.14 & 2.78 & 2.93 \\
400 & 27.44 & 8 & 2.63 & 3.04 \\
500 & 28.32 & 8.04 & 2.59 & 3.10 \\
\hline
\end{tabular}


Table 5-4: Results at the end of each modelled hot flow rates with the chiller

\begin{tabular}{l|llll}
\hline $\begin{array}{l}\text { Flow } \\
\text { Rate } \\
(\mathrm{kg} / \mathrm{hr})\end{array}$ & $\begin{array}{l}\text { Average } \\
\text { Evaporator } \\
\text { Temperature }\left({ }^{\circ} \mathrm{C}\right)\end{array}$ & $\begin{array}{l}\text { Stored Heating } \\
\text { Potential } \\
(\mathrm{kWh})\end{array}$ & $\begin{array}{l}\text { Electrical } \\
\text { Input } \\
(\mathrm{kWh})\end{array}$ & $\begin{array}{l}\text { Tank } \\
\text { Charging } \\
\text { COP }\end{array}$ \\
\hline 400 & 21.12 & 7.88 & 3.09 & 2.55 \\
600 & 24.83 & 7.68 & 3.26 & 2.36 \\
800 & 25.98 & 7.77 & 2.95 & 2.63 \\
1000 & 27.13 & 7.85 & 2.71 & 2.89 \\
1200 & 27.72 & 7.89 & 2.56 & 3.09 \\
\hline
\end{tabular}

These results confirm the results shown in the instantaneous graphs in the preceding figures, but also show the relationship between energy stored, energy input and COP. In both the medium temperature chiller and the standard heat pump, at lower flow rates, more energy is stored, but a disproportionately large amount of energy is required to generate this stored energy. Overall, based on direct comparison when only considering flows, higher flows are recommended on both the hot and cold side of the heat pump system.

\subsection{Ice Storage System Configuration and Charging Efficiency}

Once the interaction between the heat pump/medium temperature chiller and the sensible thermal storage system, the next step was to determine both the storage configuration and the interaction between the ice storage and the medium temperature chiller. The experimental test set-up consisted of a small tank, and a single, submerged pipe, in which the heat was extracted from the tank though the heat transfer between the submerged pipe and chilled glycol flowing through the pipe. Although this was effective for validating the developed model, in an actual application, a bank of submerged pipe would be utilized, multiplying the potential heat transfer from the chilled fluid.

The first step in utilizing the ice storage is determining the minimum required pipes to be installed within the storage tank to ensure enough heat transfer can be achieved 
throughout the charging process. This sizing must be complete for each individual chiller connected to an ice storage as each chiller has its own heat transfer profile based on the return temperature from the thermal storage. For the further comparison to the sensible cold storage, a fluid volume of $270 \mathrm{~L}$ was first utilized to determine the required number of pipes. This volume can later be increased, to increase the available storage capacity of the thermal storage.

To determine the number of pipes, a few assumptions were made. The first is that a rectangular array of pipes would be utilized, meaning that not just any number of pipes could be utilized, but only the total number of pipes that would make a rectangular array. The second condition is that, based on the performance and capabilities of the medium temperature chiller, the chiller will turn off if the entering fluid temperature is lower than $-8^{\circ} \mathrm{C}$. The third condition is that the system will stop charging once a total of $10 \mathrm{kWh}$ is stored within the $270 \mathrm{~L}$ tank, between sensible and latent storage. Finally, all scenarios were modelled with a condenser inlet temperature of $20^{\circ} \mathrm{C}$ and a flow rate of $18 \mathrm{~L} / \mathrm{min}$, while the evaporator had a flow rate of $14 \mathrm{~L} / \mathrm{min}$. These represent the conditions in which the chiller would produce the greatest cooling potential and as such, the conditions in which the thermal storage must be sized to.

To determine the piping layout, one additional assumption was made to simplify the parametric study, and that was to fix the rectangular array as a square array to reduce the total number of combinations that could be tested. Since the total number of pipes dictates the heat transfer that can occur into the thermal storage, the array configuration has no influence in overall performance, just the number of pipes contained in the system. The array configuration would influence the final geometry of the system, and when 
fabricated an eventual full system, further consideration would be required. The modelling results for the configuration of $6 \times 6,8 \times 8,10 \times 10,11 \times 11,12 \times 12,13 \times 13$ and $14 \times 14$ are presented in Table 5-5.

Table 5-5: Summary of results for different pipe configurations

\begin{tabular}{l|lllll}
\hline $\begin{array}{l}\text { Piping } \\
\text { Array }\end{array}$ & $\begin{array}{l}\text { Total } \\
\text { Pipes }\end{array}$ & $\begin{array}{l}\text { Total Cooling } \\
\text { Stored }(\mathrm{kWh})\end{array}$ & $\begin{array}{l}\text { Time Heat } \\
\text { Pump Ran }(\mathrm{h})\end{array}$ & $\begin{array}{l}\text { Ending Return } \\
\text { Temperature }\left({ }^{\circ} \mathrm{C}\right)\end{array}$ & $\begin{array}{l}\text { Design Goals } \\
\text { Achieved }\end{array}$ \\
\hline $6 \times 6$ & 36 & 3.00 & 0.46 & -6 & No \\
$8 \times 8$ & 64 & 5.41 & 0.77 & -6 & No \\
$10 \times 10$ & 100 & 6.30 & 0.86 & -6 & No \\
$11 \times 11$ & 121 & 10.00 & 1.43 & -4.63 & Yes \\
$12 \times 12$ & 144 & 10.04 & 1.42 & -3.47 & Yes \\
$13 \times 13$ & 169 & 10.05 & 1.41 & -2.61 & Yes \\
$14 \times 14$ & 196 & 10.05 & 1.40 & -1.97 & Yes \\
\hline
\end{tabular}

Based on these results, it was determined that a minimum of approximately 120 pipes is required to obtain the required heat transfer into the cold ice storage. Although this is the minimum number of pipes, the ending return temperature to the chiller from the ice storage is very close to the cut off temperature for the chiller, so although it meets the minimum technical requirements, the cut off temperature could easily be met if greater cooling potential is to be stored. As a result, it was decided that a $12 \times 12$ pipe configuration would be used going forward, allowing for additional cooling capacity to be stored then the initially designed $10 \mathrm{kWh}$.

To achieve the $10 \mathrm{kWh}$ stored cooling capacity, approximately $40 \mathrm{~kg}$ of ice had to be formed, which is only converting $15 \%$ of the water in the thermal storage to ice. As 
such, larger storage capacity set-points were modelled, and the results are presented in Table 5-6.

Table 5-6: Impact of different storage charge levels using the ice storage system

\begin{tabular}{l|lllll}
\hline $\begin{array}{l}\text { Storage } \\
\begin{array}{l}\text { Set-Point } \\
(\mathrm{kWh})\end{array}\end{array}$ & $\begin{array}{l}\text { Ice } \\
\text { Formed } \\
(\mathrm{kg})\end{array}$ & $\begin{array}{l}\text { Average Ice } \\
\text { Thickness } \\
(\mathrm{mm})\end{array}$ & $\begin{array}{l}\text { Percent Ice } \\
\text { Formation } \\
(\%)\end{array}$ & $\begin{array}{l}\text { Storage } \\
\text { Density } \\
\left(\mathrm{kWh} / \mathrm{m}^{3}\right)\end{array}$ & $\begin{array}{l}\text { Ending Return } \\
\text { Temperature } \\
\left({ }^{\circ} \mathrm{C}\right)\end{array}$ \\
\hline 10 & 40.06 & 4.22 & 14.8 & 37.0 & -3.47 \\
12 & 61.55 & 5.89 & 22.8 & 44.4 & -3.65 \\
14 & 83.04 & 7.37 & 30.8 & 51.9 & -3.81 \\
16 & 104.80 & 8.71 & 38.8 & 59.3 & -3.94 \\
18 & 126.28 & 9.93 & 46.8 & 66.7 & -4.05 \\
20 & 147.76 & 11.02 & 54.7 & 74.1 & -4.15 \\
\hline
\end{tabular}

When compared to the value of water, assuming the same $20^{\circ} \mathrm{C}$ reference temperature and that a tank temperature of $4^{\circ} \mathrm{C}$ can be reached, water has a storage density of $18.6 \mathrm{kWh} / \mathrm{m}^{3}$ and the glycol solution, chilled to $-8^{\circ} \mathrm{C}$ has a storage density of $28.6 \mathrm{kWh} / \mathrm{m}^{3}$. This shows that the ice storage is able to achieve storage densities which are far superior to the storage densities achieved using the sensible storage densities. This proves that the ice storage can solve the problem of the required storage capacity, in much low volumes. To store $20 \mathrm{kWh}$ using water as the storage medium, $1070 \mathrm{~L}$ is required.

Although the model will allow for $100 \%$ of the water to become ice, a certain percentage of the tank must remain water to allow for the flow of the cooling water to the air handling unit to provide space cooling. Based on literature and the geometry of the tank and piping layout, an assumption has been made that the maximum amount of ice formation is $50 \%$. This allows enough fluid to be pumped through the storage and air handling units to meet the cooling demand, and ensuring there is enough space for ice to form without causing damage to the system through the expansion. Additionally, it is important to ensure the quantity of ice will fit between the pipe layout. The ice does not form equally along the 
length of the pipe, with a greater amount of ice forming in the first node of the pipe, compared to the exiting node, as the flowing fluid is at a lower temperature before heat is removed from the thermal storage as it flows down the length of the pipe. With a volume of $270 \mathrm{~L}$, and using the pipe length of $1.3 \mathrm{~m}$, the tank must be $46 \mathrm{~cm} \times 46 \mathrm{~cm}$. Using the $12 \times 12$ pipe layout, the pipe would be located at a spacing, center of pipe to center of pipe of $3.83 \mathrm{~cm}$. The radius of the pipe is $0.635 \mathrm{~cm}$, meaning the spacing between the outside of the two pipes is $2.56 \mathrm{~cm}$, or meaning the maximum ice thickness that can be built up on each pipe, under this configuration is $12.8 \mathrm{~mm}$. When looking at the results from the previous parametric study, the use of $20 \mathrm{kWh}$ was removed as it exceeds the $50 \%$ ice formation assumption. When the $18 \mathrm{kWh}$ results were examined, the ice thickness of the first node is $13.7 \mathrm{~mm}$, so this would not fit within the geometry of the tank. From the results, in this case, the $12.8 \mathrm{~mm}$ maximum ice thickness is achieved at $16.8 \mathrm{kWh}$ of cooling potential stored, so in this case, $16 \mathrm{kWh}$ is the maximum cooling potential that can be stored in the $270 \mathrm{~L}$ tank. Larger quantities can be stored in a greater volume tank, with the same number of submerged pipes as the spacing between each pipe would increase.

\subsection{Comparison between Sensible and Latent Cold Storage Efficiency}

Once the required storage configurations and set points were determined for both the sensible and latent cold thermal storages, a comparison was required to determine when each storage system is most beneficial. Due to the lower achievable temperatures and higher storage capacity, the water/glycol solution was used to represent the sensible storage. Both the sensible and latent storages were only modelled with the medium temperature chiller, to ensure an accurate comparison. The sensible storage was run until the return temperature was $-8^{\circ} \mathrm{C}$ and the ice storage was run until a total of $16 \mathrm{kWh}$ was 
stored. The condenser side of the chiller was held constant for both systems at $18 \mathrm{~L} / \mathrm{min}$ and $20^{\circ} \mathrm{C}$ inlet conditions. The total energy stored, the return temperature to the chiller, and the charge efficiency were compared, using the graphs in Figure 5-5 and Figure 5-6.

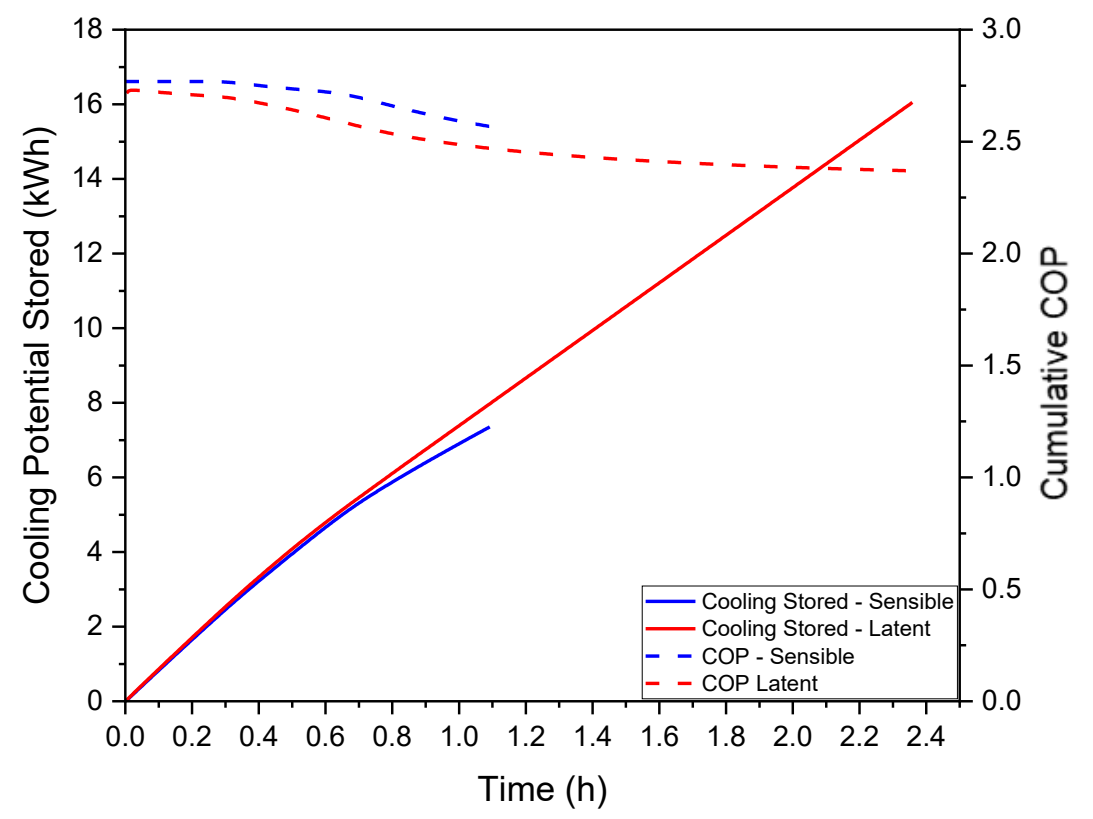

Figure 5-5: Comparison of cooling potential stored and COP between sensible and latent storage

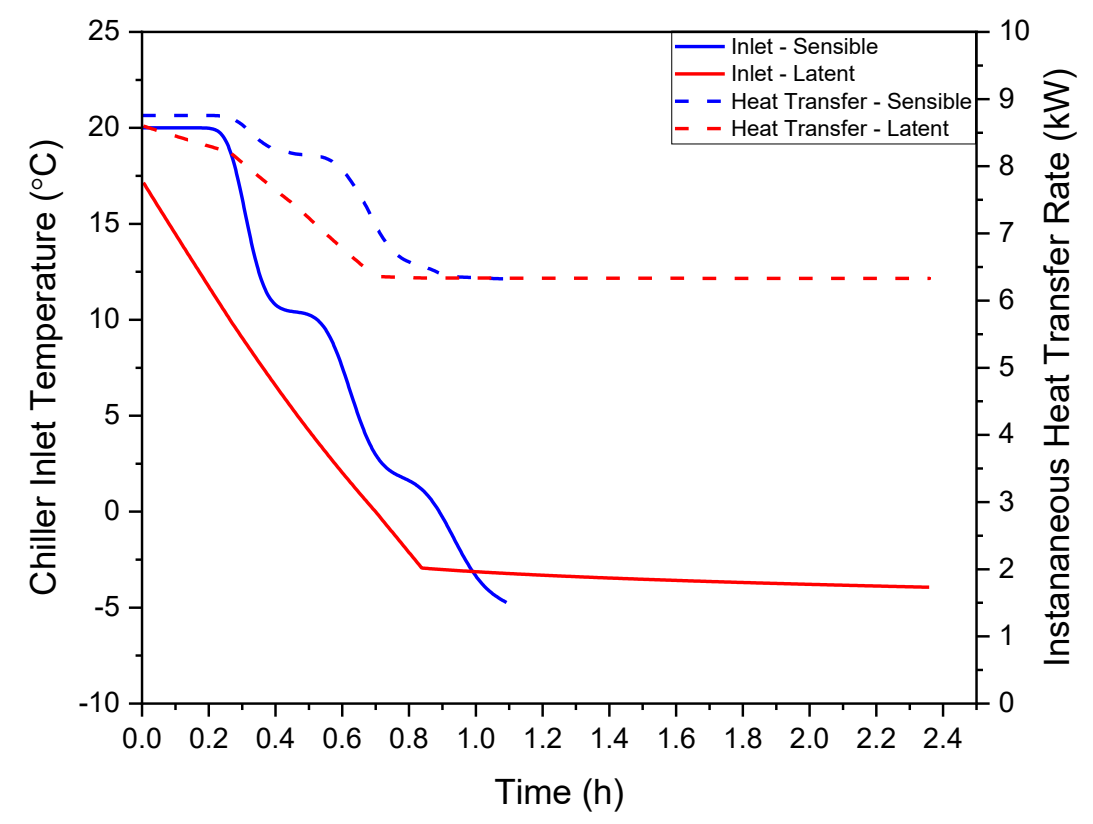

Figure 5-6: Comparison of inlet temperature and heat transfer rates between sensible and latent storage 
Based on these results, a few conclusions can be drawn and utilized going forward through the design process and when looking at annual simulations of the system. The first is, at lower storage requirements, the sensible storage system can be charged at a high COP. This is because the return temperatures are higher, improving chiller performance, particularly while the tank is stratified. That being said, the amount of energy that can be stored, with no significant decrease in the COP to the overall system, is significantly higher in the ice storage. Even if lower amount of cooling potential needed to be stored, the small degradation in charge performance does not justify running parallel systems. Overall, it can be seen that the ice storage has a similar storage profile to that of the sensible storage but allows for a significant increase in cooling potential to be stored, at the cost of a much more complex system. As such, the ice storage should only be used when higher storage capacity, with space limitation, is required.

\subsection{Conclusions}

Based on the results of modelling in individual storage systems, it was determined that in all cases, higher flow rates should be utilized between the heat pump/medium temperature chiller and the thermal storage system. The increased heat transfer as a result of the higher flow rates is more advantageous than the benefits of higher inlet temperatures that are achievable using a stratified storage tank. While using the chiller studied within this study, a minimum of 120 heat transfer pipes is required, however, it is recommended a minimum of 140 pipes be utilized so that larger storage capacities could be obtained. Finally, at $270 \mathrm{~L}$, the maximum storage based on geometry is $16 \mathrm{kWh}$, however, with larger volumes, and greater spacing between the pipes, larger storage capacities could be easily

achieved. When comparing the ice storage to the sensible storage, similar performance is 
seen at lower storage capacity, however, the ice storage continues to charge at a similar performance and allows for much larger storage capacity for the same tank volume. 


\section{Chapter 6: Annual Modelling Study}

Once the individual component models were developed and validated, and the performance of sub-systems and component interaction was optimized, the next step was to develop the complete system model and couple the system model with a house model. This complete model of the different heat/pump thermal storage coupled to a modelled house will be used to determine the potential benefits of offsetting peak residential electrical consumption. This chapter first describes the house models that were developed to assess the annual performance of the system, and the baseline energy consumption of these modelled buildings. The development of the system models and control strategies is then described, and finally, the interaction between the heat pump or chiller and the thermal storage systems are modelled and the optimal configurations determined.

\subsection{House Model Development}

Before the proposed system could be modelled to determine the annual performance, a house model had to be developed in TRNSYS to provide building loads for the heating and cooling system. The house was modelled to represent a newly built house located in Ottawa, Ontario, Canada. The modelled house is a two-story, single detached house with a full basement, and the design was based off of the Ontario Building Code and National Energy Code $[79,80]$. Each level has a floor area of $110 \mathrm{~m}^{2}$ with a total volume of $835 \mathrm{~m}^{3}$. The house was broken into 4 air zones, being the basement, main floor, $2^{\text {nd }}$ floor and the attic, with air exchange between the basement, main and $2^{\text {nd }}$ floor. The heating was an air based system, where $20 \%$ of distribution air goes to the basement, $35 \%$ to the main floor and $45 \%$ the $2^{\text {nd }}$ floor, allowing the increased heating and cooling demand in the $2^{\text {nd }}$ floor to be met as a result of the heat loss through the ceiling into the attic. A single 
thermostat has been placed in the main floor with a heating set-point of $20^{\circ} \mathrm{C}$ and a cooling set-point of $23^{\circ} \mathrm{C}$. The remaining specifications for building insulation and windows are provided in Table 6-1.

Table 6-1: House model parameters

\begin{tabular}{l|lcc}
\hline Type & Parameter & Units & Value \\
\hline \multirow{4}{*}{ Thermal Resistance } & Above Grade Walls & $\mathrm{m}^{2} \mathrm{~K} / \mathrm{W}$ & 4.5 \\
& Attic & $\mathrm{m}^{2} \mathrm{~K} / \mathrm{W}$ & 11.5 \\
& Below Grade Walls & $\mathrm{m}^{2} \mathrm{~K} / \mathrm{W}$ & 2.7 \\
& Under Slab & $\mathrm{m}^{2} \mathrm{~K} / \mathrm{W}$ & 1.9 \\
\hline \multirow{2}{*}{ Windows } & U-Value & $\mathrm{W} / \mathrm{m}^{2} \mathrm{~K}$ & 1.27 \\
& Solar Heat Gain & & 0.624 \\
\hline \multirow{4}{*}{ House } & Air Leakage & $\mathrm{ACH}$ & 0.05 \\
& Window to Wall Ratio & $\%$ & 30 \\
& Occupancy & Number & 4 \\
& & of People & 4 \\
\hline
\end{tabular}

\subsubsection{Baseline Energy Consumption}

Once the house model was developed within TRNSYS, a baseline energy consumption for heating and cooling had to be determined. A fluid heater and fluid cooler, both with a COP of 1 and with no losses, were integrated into the model to represent an ideal heating and cooling system. The model was then run for 1 year ( 8760 hours), with the electrical consumption recorded every 5 minutes. Based on these simulation results, the house was found to have an annual space heating load of $16,215 \mathrm{kWh}$ and an annual cooling load of $4686 \mathrm{kWh}$. Of more importance than the total energy consumption was the time at which the energy was used, the cost to the consumer and the total greenhouse gas emissions as a result of the space heating and cooling. The energy consumption was broken down by peak, mid-peak and off-peak as defined by Ontario's time of use billing [81], while the 
greenhouse gas emissions were calculated using the average for each time period for winter and summer periods. A summary of these results is presented in Table 6-2.

Table 6-2: Baseline energy consumption by time, heating costs and greenhouse gas emissions

\begin{tabular}{|c|c|c|c|c|c|c|c|c|}
\hline \multirow{2}{*}{$\begin{array}{l}\text { Energy } \\
\text { Type }\end{array}$} & \multicolumn{3}{|c|}{ Summer (kWh) } & \multicolumn{3}{|c|}{ Winter (kWh) } & \multirow{2}{*}{$\begin{array}{c}\text { Cost } \\
(\mathrm{CAD} \$)\end{array}$} & \multirow{2}{*}{$\begin{array}{l}\text { Greenhouse } \\
\text { Gas Emissions } \\
(\mathrm{kg})\end{array}$} \\
\hline & Peak & $\begin{array}{l}\text { Mid- } \\
\text { Peak }\end{array}$ & $\begin{array}{l}\text { Off- } \\
\text { Peak }\end{array}$ & Peak & $\begin{array}{l}\text { Mid- } \\
\text { Peak }\end{array}$ & $\begin{array}{l}\text { Off- } \\
\text { Peak }\end{array}$ & & \\
\hline Heating & 17 & 87 & 2091 & 1975 & 752 & 11,293 & 1580 & 321 \\
\hline Cooling & 2399 & 857 & 1430 & 0 & 0 & 0 & 66 & 871 \\
\hline Total & 2416 & 944 & 3521 & 1975 & 752 & 11,293 & 2444 & 1192 \\
\hline
\end{tabular}

Based on these results, a number of conclusions can be noted. The baseline energy consumption for space heating is predominantly off peak, with over $80 \%$ of the winter space heating load occurring during off-peak periods. This was expected, as during the overnight period, no solar gains are present to reduce the space heating load and the outdoor temperature is at its lowest. This results in the bulk of the heating required during the overnight, off-peak period. If a night-time temperature set-back was introduced, the energy consumption would shift towards a larger percentage during peak periods, particularly if the morning heating occurs at or after $7 \mathrm{am}$. Although a large percentage of the heating load occurs during off peak periods, almost $2000 \mathrm{kWh}$ of heating occurs during peak periods, accounting for almost $13 \%$ of the total space heating costs, of which most occurs early in the morning. This peak load has the potential to be shifted to off-peak periods through the use of thermal storage overnight.

When looking at the space cooling demand, a much larger percentage of the load occurs during peak periods, with $51 \%$ of the cooling load (and $65 \%$ of cost) occurring during peak periods, while peak periods only account for $18 \%$ of the summer period. As such, space cooling shows the greatest potential to see a meaningful benefit from shifting 
electricity consumption from peak to off-peak periods using heat pumps and thermal storage.

\subsubsection{Baseline Energy Consumption Using a Heat Pump}

The previous section indicated the total space heating and cooling loads, and the time of day they occurred using a generic auxiliary heater and chiller with a COP of 1 . This provided useful data to recognize energy trends and a true baseline, however to make a true comparison of the proposed system that integrates both a heat pump and thermal storage systems, a baseline energy consumption using the same heat pump that will be integrated with the thermal storage had to be determined. Using the same house model and air distribution system, a $6 \mathrm{~kW}_{\text {thermal }}$ heat pump was coupled with the heating and cooling coils. The single heat pump as experimentally tested did not have the heating and cooling capacity to meet the building loads of the modelled house, and therefore all values (flow rates, power consumption and heat transfer rates) were all scaled by a factor of two. This work focused on the heat pump and thermal storage systems and not the potential heat source or sink for the system, and as such to remove any discrepancies or errors that could be introduced by these, a constant heat source was provided on the source side of $15^{\circ} \mathrm{C}$ and $900 \mathrm{~kg} / \mathrm{hr}$ during the heating season and cooling water equal to the outdoor dry bulb temperature during the cooling season. The same thermostat set-points were used as for the baseline energy consumption, and the electrical consumption for space heating and space cooling were independently reordered and presented in Table 6-3. 
Table 6-3: Baseline energy consumption using a heat pump for space heating and cooling

\begin{tabular}{|c|c|c|c|c|c|c|c|c|}
\hline \multirow{2}{*}{$\begin{array}{l}\text { Energy } \\
\text { Type }\end{array}$} & \multicolumn{3}{|c|}{ Summer (kWh) } & \multicolumn{3}{|c|}{ Winter $(\mathrm{kWh})$} & \multirow{2}{*}{$\begin{array}{c}\text { Cost } \\
(\mathrm{CAD} \$)\end{array}$} & \multirow{2}{*}{$\begin{array}{c}\text { Greenhouse } \\
\text { Gas Emissions } \\
(\mathrm{kg})\end{array}$} \\
\hline & Peak & $\begin{array}{l}\text { Mid- } \\
\text { Peak }\end{array}$ & $\begin{array}{l}\text { Off- } \\
\text { Peak }\end{array}$ & Peak & $\begin{array}{l}\text { Mid- } \\
\text { Peak }\end{array}$ & $\begin{array}{l}\text { Off- } \\
\text { Peak }\end{array}$ & & \\
\hline Heating & 8 & 27 & 599 & 356 & 101 & 2742 & 373 & 205 \\
\hline $\begin{array}{l}\text { Cooling } \\
\text { Hot }\end{array}$ & 681 & 288 & 399 & 43 & 106 & 26 & 219 & 104 \\
\hline Water & 196 & 1012 & 838 & 1147 & 222 & 967 & 562 & 265 \\
\hline Total & 885 & 1326 & 1836 & 1546 & 429 & 3735 & 1154 & 574 \\
\hline
\end{tabular}

These results held the trends of the first baseline energy consumption; however, the introduction of the heat pump saw the energy consumption reduced by $78 \%$ for space heating and by $77 \%$ for space cooling. Additionally, the required energy and costs to meet the building's domestic hot water needs were also modelled and presented. This uses a standard, electric resistance on-demand heating coil. The values obtained from this base model incorporating the heat pump with no thermal storage will be used as the baseline for all future simulations incorporating thermal storage.

The liquid to liquid heat pump using a set source temperature and liquid based coils to supply heating and cooling was selected as it provided the most comparable system to the proposed system using thermal storage that will be compared to these values. Other systems could have been selected as the base case utilizing a heat pump as the primary source of both heating and cooling of the building. This could be either through the use of a standard air source heat pump with an electric back-up heating system for the coldest periods of the year, or a cold climate air source heat pump that would work throughout the winter. In both of these cases, because of the much lower source temperatures being used as opposed to the liquid based heat pump, would see much higher base load heating values. This would provided a higher energy and cost baseline to compare future systems against, 
but were no selected as it was decided that the liquid based systems provided a more accurate comparison to the future systems tested.

\subsection{Heating and Cooling System Model}

Once the individual components of the system were validated in TRNSYS, a complete model of the proposed system was developed, including the hot and cold thermal storage, the heat pump, heat source and sinks, and the system controls. This model was built to characterize and optimize the interaction of the heat pump with the thermal storage systems. This study focuses on the systems itself, and not the heat sink and heat source and as such, a constant supply of $15^{\circ} \mathrm{C}$ water was used as source input to the heat pump during the heating season. On the heat sink side during the cooling season, the outdoor ambient temperature was used. A control strategy was implemented so that if either the hot thermal storage or the cold thermal storage tank had reached their desired set-point, but the other thermal storage was still being charged, the fluid would bypass the storage tank for either the heat sink or source. A schematic of the control logic is shown in Figure 6-1, while images of all of the TRNSYS models used within this study are shown in Appendix . 


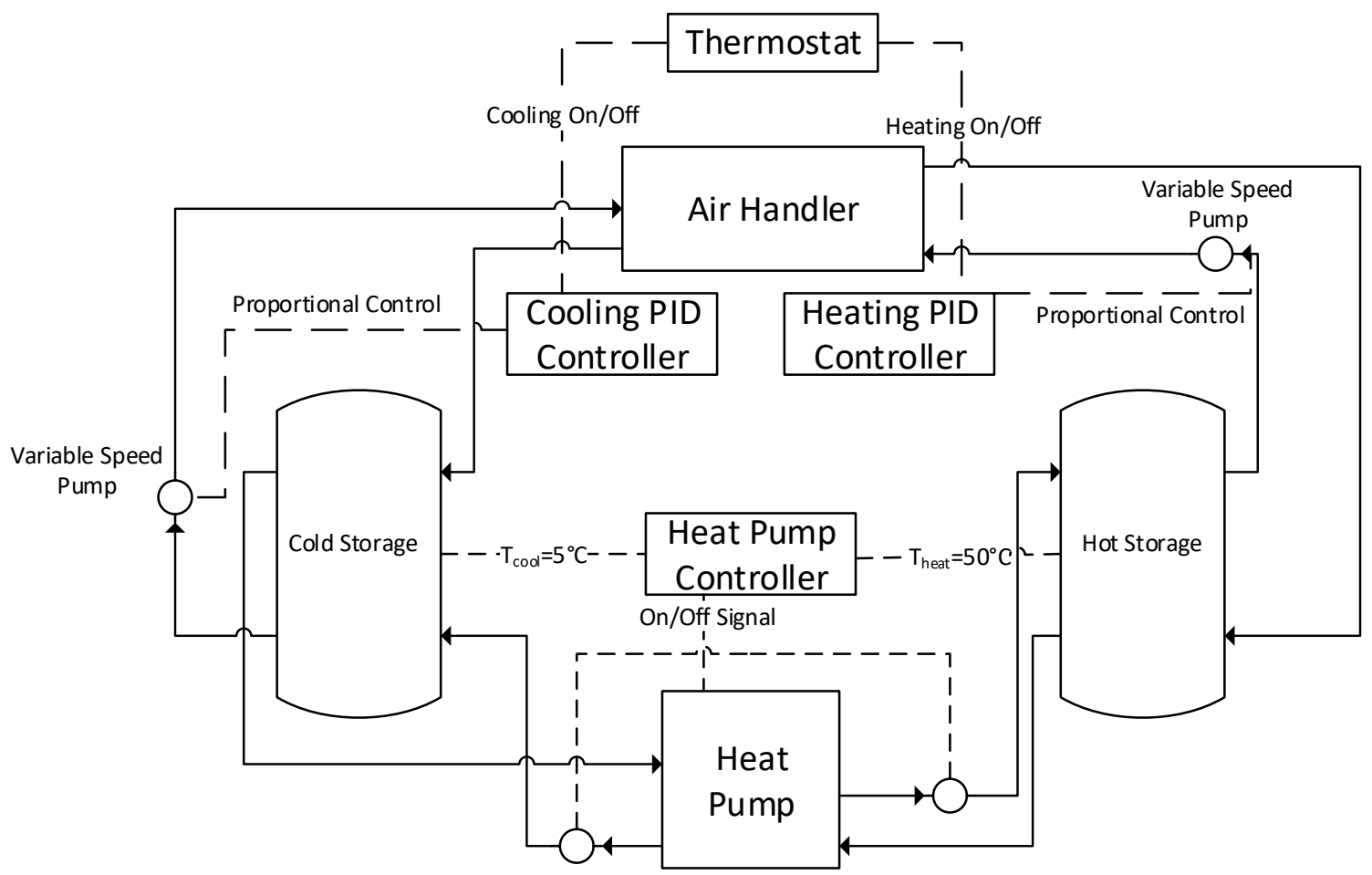

Figure 6-1: Control schematic for the proposed system as modelled

\subsection{Integration of Hot and Cold Thermal Storage}

The first iteration to determine the potential for demand side management with a heat pump and thermal storage utilized a standard ground source heat pump and both a hot and cold thermal storage with water as the hot storage medium and the glycol/water solution for the cold storage. To determine the optimal configuration, simulations were run for a range of source flow rates and tank set-points. Additionally, these results would be used to confirm the results observed when just examining the individual systems and their interaction. The first iteration simply inserted the tanks and used the temperature of the tank, at a location $80 \%$ from the bottom for the hot tank, and $20 \%$ of the way from the bottom for the cold tank. This set-point was held constant at first at $50^{\circ} \mathrm{C}$ for the hot tank and $0^{\circ} \mathrm{C}$ for cold tank to determine the impact of flow on the annual simulations. The results 
of this parametric study, looking at simultaneously the change in hot and cold flow are showing in Figure 6-2.

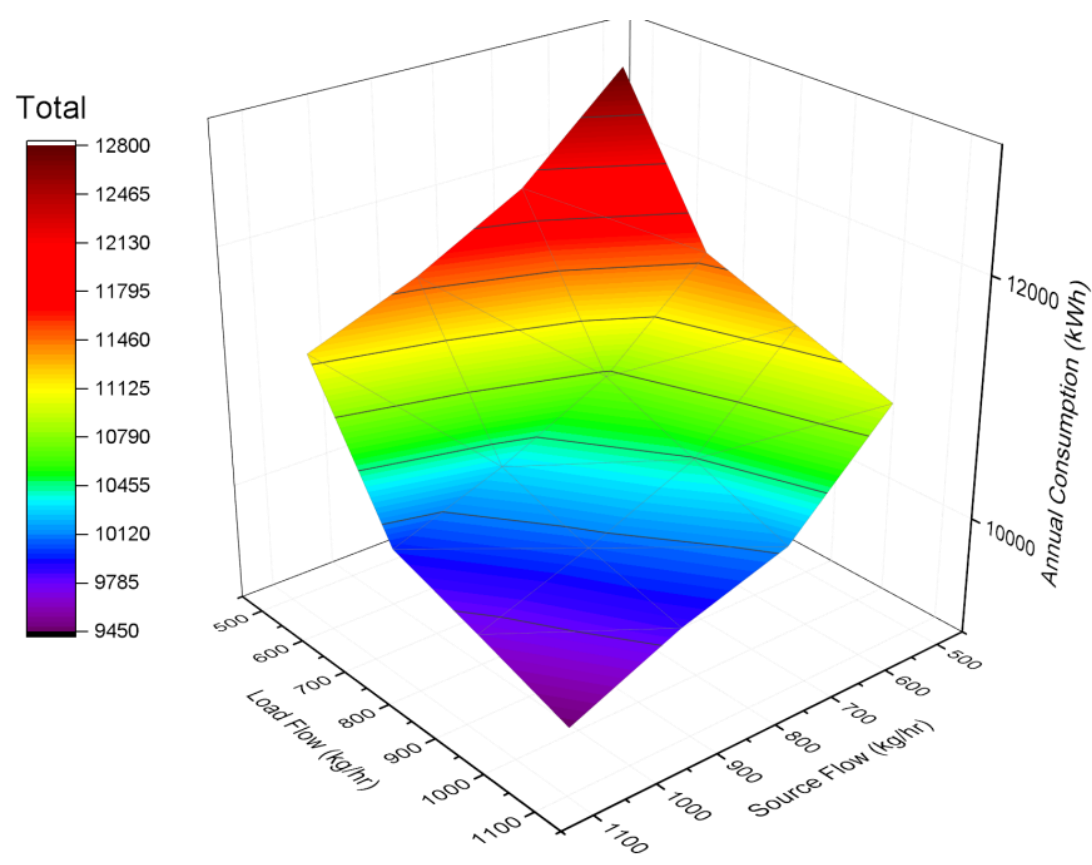

Figure 6-2: Simulation results for varying source and load flow rates

These results confirmed what was seen when examining the impact of flow on the individual components, and on an annual basis, it is confirmed that higher flow rates have a significant impact on the amount of energy required to meet the heating and cooling demand. As the flow rates on both the source and load side of the heat pump decreased, the total annual energy consumption rose significantly when compared to using flow rates on both the load and source side. This reaffirmed the results found in the short term laboratory studies previously discussed.

The second scenario that was modelled was the impact of the tank set-points on the annual energy consumption to meet heating and cooling demands. The cold side set-point 
was varied from $-5^{\circ} \mathrm{C}$ to $15^{\circ} \mathrm{C}$ and the hot side set-point was varied from $30^{\circ} \mathrm{C}$ to $60^{\circ} \mathrm{C}$. The results are shown in in Figure 6-3.
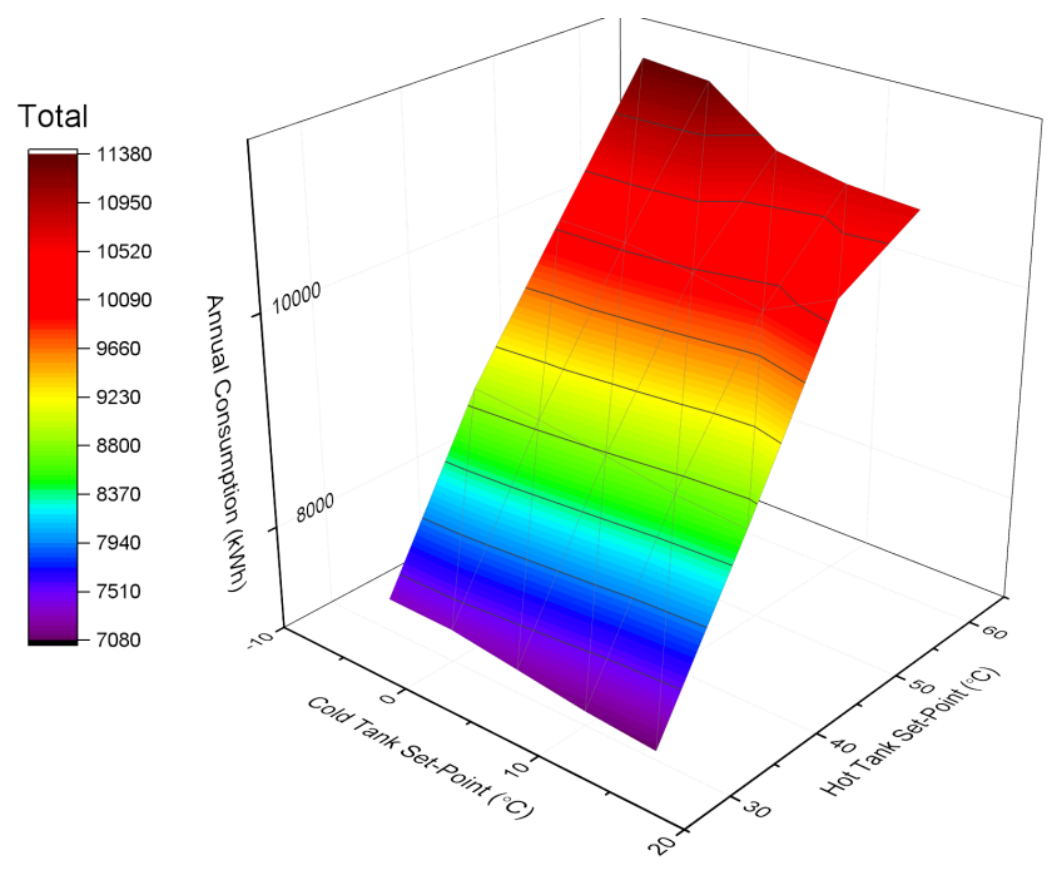

Figure 6-3: Simulation results for varying hot and cold tank set-points

These tests were not intended to offset peak loads but determine the impact of some of the main variables on the annual energy consumption to meet the space heating and cooling demand. From the results of the varying set-points, it was observed that the hot side set-point had a much more significant impact than the impact of the cold side set-point. This showed that it may be easier to store and off-set peak cooling potential, as using a lower set-point, and consequently being able to increase storage capacity. In comparison, the increase in hot temperature set-point which allows for higher storage capacities resulted in a significant increase in annual energy consumption. This on its own is not conclusive 
and requires additional testing and verification to determine the true impact of the thermal storages on the overall performance.

\subsection{Basic Set-Point Control of the Thermal Storage Systems}

Simply adding thermal storage to the system, on a continuous set-point no matter the time of day had no impact on reducing mid-peak and peak consumption. This is the result that the tanks have a constant set-point, meaning as soon as some energy is consumed, the tank temperature drops and triggers the heat pump, no matter the time of day. As such, these results were used to determine the optimal configuration for off-peak periods, but proved that different set-points are required for mid-peak/peak periods and off-peak periods. Using these results, the tank set-points for mid-peak and peak periods was set at $30^{\circ} \mathrm{C}$ for the hot tank and $15^{\circ} \mathrm{C}$ for the cold tank. A parametric study was then run varying the set-points during off-peak periods from $30^{\circ} \mathrm{C}$ to $60^{\circ} \mathrm{C}$ for the hot tank and $-5^{\circ} \mathrm{C}$ to $15^{\circ} \mathrm{C}$ for the cold tank. The results of these simulations are shown in Figure 6-4, which looked at the annual energy consumption and peak/mid-peak consumption for the house model used. 

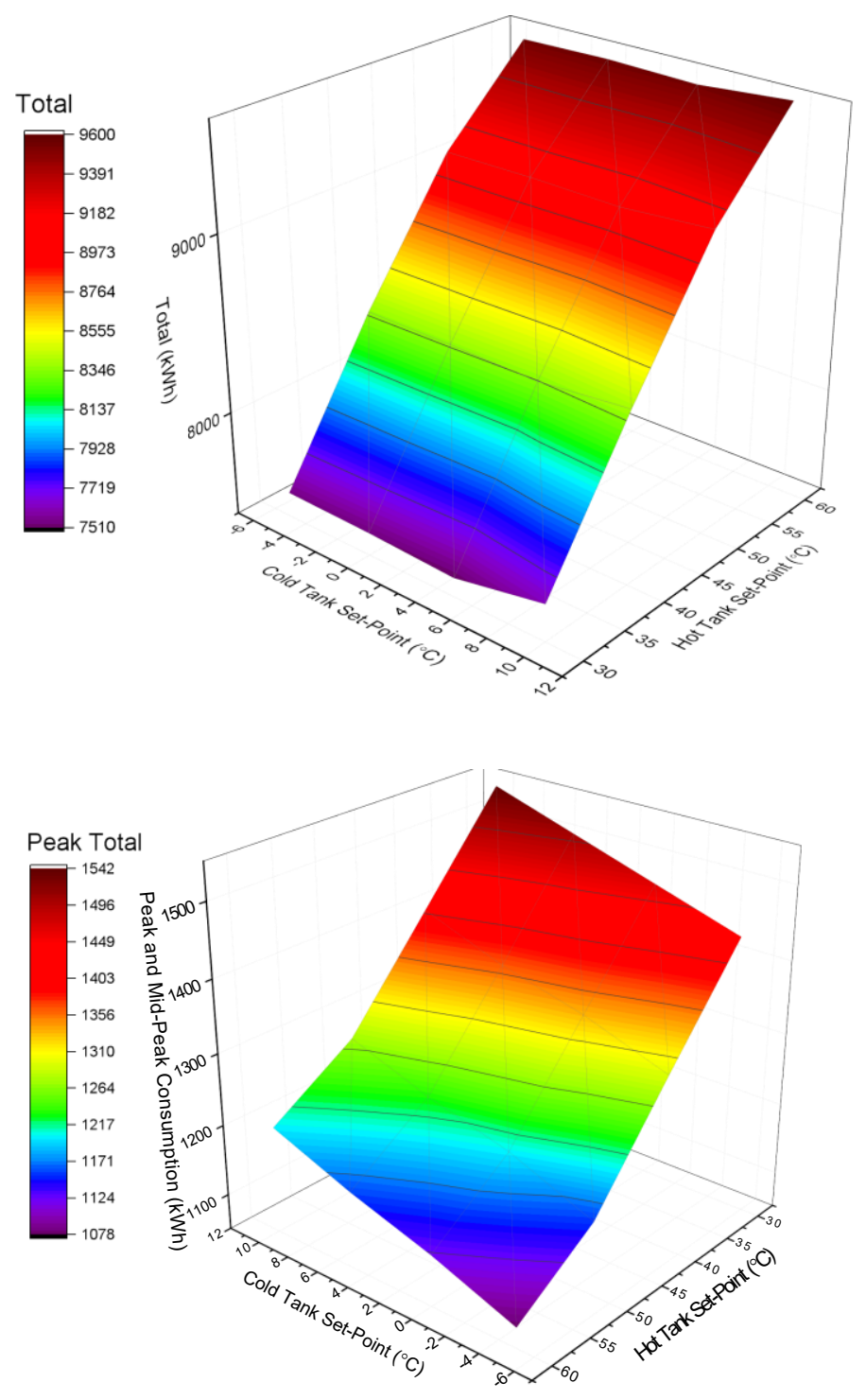

Figure 6-4: Simulation results varying off-peak tank set-points

From these results, every permutation of off-peak set-points reduces the amount of energy consumed during peak and mid-peak periods, however, the total energy consumption increases. This is most notable in terms the impact of the hot tank peak setpoint as it influences the heating of house which is the much large proportion of the load. In every scenario, when the set-point is increased for the hot tank or decreased for the cold tank, the total energy consumption and annual operating costs is significantly increased when compared to the base cases. Additionally, even when the set-point of the tank is at 
its extreme, there is still a significant peak and mid-peak electrical load to meet the annual heating and cooling demand. This indicated that the storage capacity of both the hot and cold thermal storage systems was too small to meet the peak and mid-peak demand, and system with a greater storage capacity should be investigated. As such, the tank volume for both thermal storage systems were varied between the base case (270L cold storage and $300 \mathrm{~L}$ hot storage), up to a maximum size of $2000 \mathrm{~L}$. The results of these simulations for annual total energy consumption, peak and mid-peak consumption and the annual electrical costs are shown in Figure 6-5 to Figure 6-7.

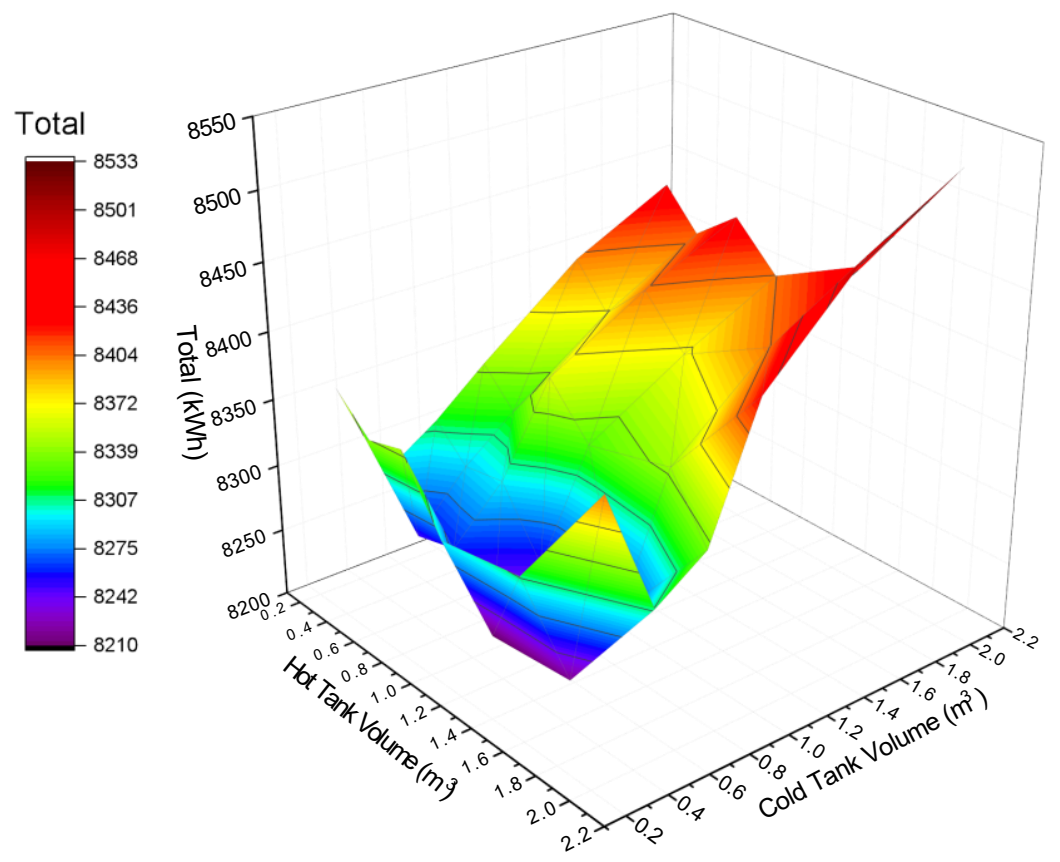

Figure 6-5: Impact of changing thermal storage volumes on total energy consumption 


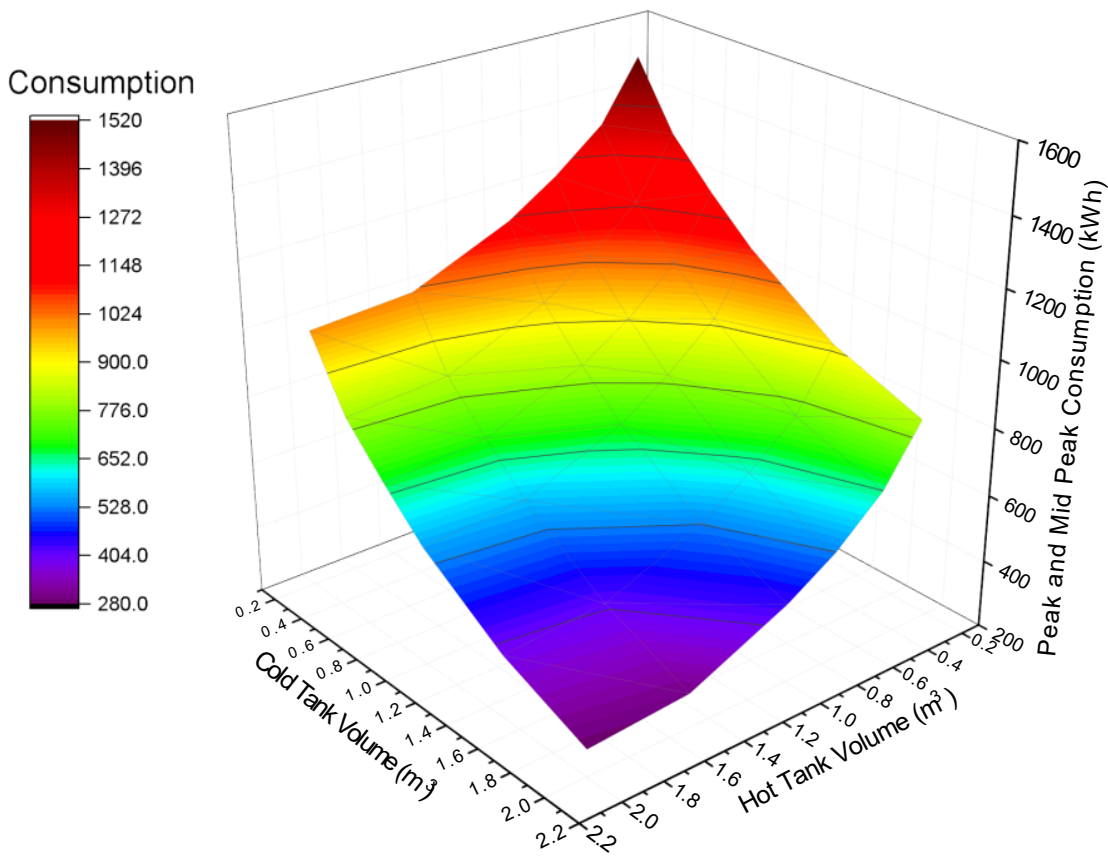

Figure 6-6: Impact of changing thermal storage volumes on peak electrical consumption
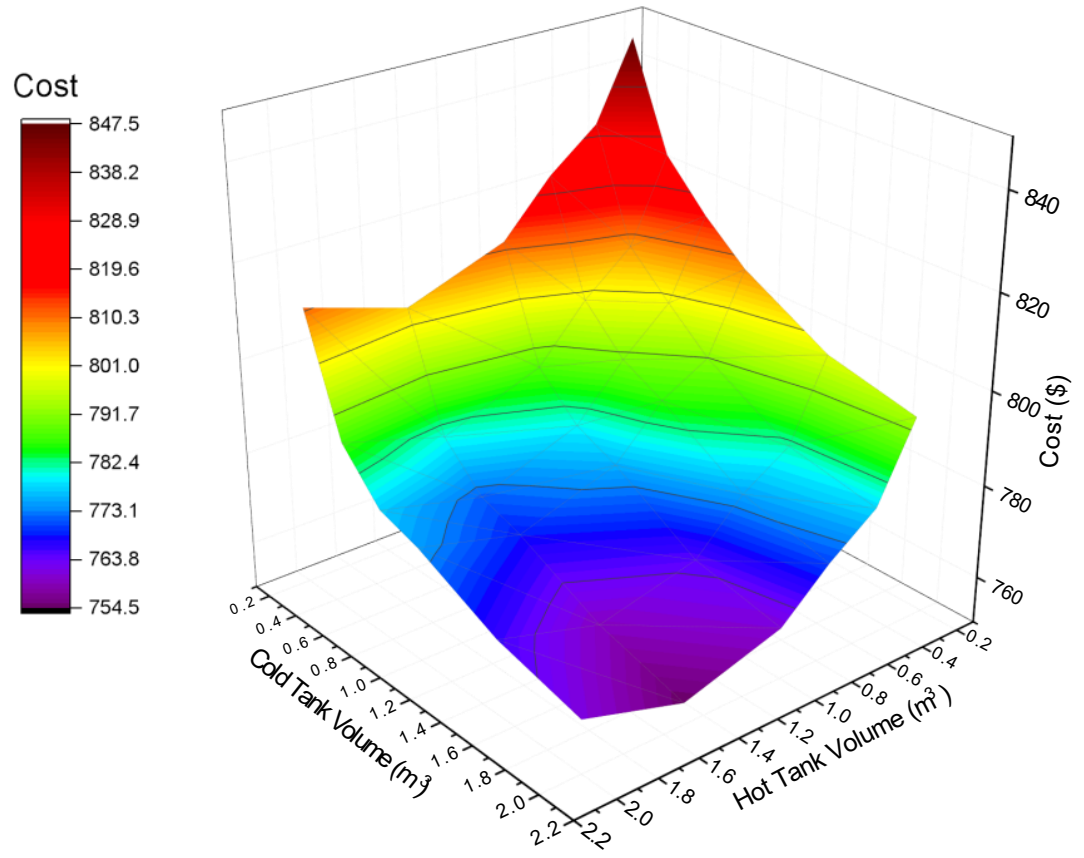

Figure 6-7: Impact of changing thermal storage volumes on annual utility costs

As expected, as the storage capacity of the tanks increases, the total annual energy consumption increases, as the heat pump operates under less ideal conditions. Although total consumption increased, the peak and mid-peak consumption decreases significantly, 
and as a result, the annual electrical costs also decreased as the tank sizes increase. When compared to the base case where no thermal storage systems are used, the annual operating costs are still $17 \%$ and $22 \%$ higher using the thermal storage system. This is predominantly a result of the significant increase in off-peak electrical consumption as a result of the decreased evaporator temperatures during cooling and increased condenser temperatures during heating. When using the scenario where $2000 \mathrm{~L}$ storage tanks are used for both thermal storage systems, the reductions in peak and mid-peak consumption and resulting increase in total consumption are provided in Table 6-4.

Table 6-4: Results of the optimal configuration looking at just heating and cooling

\begin{tabular}{l|cccc}
\hline Simulation & $\begin{array}{c}\text { Total } \\
\text { Electrical } \\
\text { Consumption } \\
(\mathrm{kWh})\end{array}$ & $\begin{array}{c}\text { Peak and Mid- } \\
\text { Peak } \\
\text { Electrical } \\
\text { Consumption } \\
(\mathrm{kWh})\end{array}$ & $\begin{array}{c}\text { Annual Cost } \\
(\$)\end{array}$ & $\begin{array}{c}\text { Greenhouse } \\
\text { Gas Emissions } \\
\left(\mathrm{kg} \mathrm{CO}_{2}\right)\end{array}$ \\
\hline $\begin{array}{l}\text { Base Case } \\
\text { Optimized }\end{array}$ & 5375 & 1609 & 592 & 309 \\
$\begin{array}{l}\text { Thermal Storage } \\
\text { Difference }\end{array}$ & 8532 & 281 & 761 & 454 \\
\hline
\end{tabular}

From this, the reason for the increase in annual costs can be seen in that the average cost of peak and mid-peak electricity is 15.6 cents $/ \mathrm{kWh}$, while off-peak rates are 8 cents/kWh. If it is assumed that the split is equal between peak and mid-peak periods, to be cost neutral, for every $\mathrm{kWh}$ of electrical consumption saved during peak and mid-peak periods, the maximum increase in off-peak electrical consumption would be $1.95 \mathrm{kWh}$. Currently, the performance degradation is so great with thermal storage, that for each $\mathrm{kWh}$ saved, peak consumption increases $2.56 \mathrm{kWh}$. When using greenhouse gas emissions as the indicative metric, for every $\mathrm{kWh}$ of peak and mid-peak electrical consumption 
reduction, the maximum corresponding increase in peak consumption is not clear cut due to the seasonal variations, but if summer and winter periods are to be considered equal, the maximum increase cannot exceed $1.25 \mathrm{kWh}$. As such, to make this system economically and environmentally viable, the ratio between peak loading offset compared to peak loading increase must be significantly reduced.

The results of this study, using the standard heat pump coupled with sensible hot and cold thermal storage systems, was not able to adequately offset peak and mid-peak loads in an Ottawa house to be either cost or carbon emission neutral. The main issue was that the standard heat pump is optimally designed for the narrow range of temperatures typically required to directly heat and cool a residential building. This does not couple well with the cold thermal storage, as the heat pump must operate out of its design range to charge the cold storage, and as a result, operate in a sub-optimal range, increasing the required electrical input to meet the heating and cooling of the building.

To counteract this sub-optimal range, one of three strategies could be utilized. The first would be to employ a very large sensible thermal storage, where the required cooling and heating potential could be stored while not having a significant impact on the entering temperatures to the heat pump. The second would be to employ a phase change material, which changes phase close to the temperature required for cooling, in the range of $8^{\circ} \mathrm{C}$ and $12^{\circ} \mathrm{C}$, meaning the heat pump does not need to leave its optimal range, while having a higher storage density and smaller volume. The final option would be to utilize a heat pump/chiller designed for the lower temperature ranges required for the thermal storage to have adequate capacity to meet the peak and mid-peak building loads. The first of these strategies employed was to utilize a medium temperature chiller to meet the heat and 
cooling load through the thermal storage systems.

\subsection{Medium Temperature Chiller for Meeting Heating and Cooling Loads}

Once it was determined that using a standard ground source heat pump with thermal storage would not provide the performance required for the desired demand side management through the shifting of peak and mid-peak loads to off-peak periods. To obtain improved performance and allow for higher charge efficiencies, a medium temperature chiller designed to operate with lower evaporator temperatures was utilized. This chiller directly replaced the standard heat pump and the same modelling procedure was followed to determine its potential.

\subsubsection{Baseline Results Using the Medium Temperature Chiller}

The first step in testing the low temperature chiller was to determine its performance when used to directly heat and cool the building. A baseline test was run to determine the annual performance and the total electrical consumption during both the peak, mid-peak and off-peak periods over a single year for Ottawa. These results are shown in Table 6-5.

Table 6-5: Base building loads using the medium temperature chiller

\begin{tabular}{|c|c|c|c|c|c|c|c|c|c|}
\hline \multirow{2}{*}{$\begin{array}{l}\text { Energy } \\
\text { Type }\end{array}$} & \multicolumn{3}{|c|}{ Summer (kWh) } & \multicolumn{3}{|c|}{ Winter $(\mathrm{kWh})$} & \multirow{2}{*}{$\begin{array}{c}\text { Total } \\
\text { Energy Use } \\
(\mathrm{kWh})\end{array}$} & \multirow{2}{*}{$\begin{array}{l}\text { Cost } \\
(\$)\end{array}$} & \multirow{2}{*}{$\begin{array}{c}\text { GHG } \\
\text { Emissions } \\
(\mathrm{kg})\end{array}$} \\
\hline & Peak & $\begin{array}{l}\text { Mid- } \\
\text { Peak }\end{array}$ & $\begin{array}{l}\text { Off- } \\
\text { Peak }\end{array}$ & Peak & $\begin{array}{l}\text { Mid- } \\
\text { Peak }\end{array}$ & $\begin{array}{l}\text { Off- } \\
\text { Peak }\end{array}$ & & & \\
\hline Heating & 10 & 37 & 823 & 496 & 138 & 3753 & 5257 & 512 & 281 \\
\hline $\begin{array}{l}\text { Cooling } \\
\text { Hot }\end{array}$ & 867 & 376 & 513 & 55 & 131 & 33 & 1975 & 280 & 133 \\
\hline Water & 196 & 1012 & 838 & 1147 & 222 & 967 & 4382 & 562 & 265 \\
\hline Total & 1073 & 1425 & 2174 & 1698 & 491 & 4753 & 11614 & 1354 & 679 \\
\hline
\end{tabular}

Based on these base results, it can be quickly seen that the decrease in performance of the medium temperature chiller under the ideal conditions the heat pump is designed for 
caused a significant increase in energy consumption on an annual basis. The belief was that although this increase is observed when directly heating and cooling the space, there will be a much smaller decrease in performance as the thermal storage systems are added. To determine the impact of each individual system, the individual systems and thermal storages were implemented one by one to determine their potential for demand side management in the residential setting.

\subsubsection{Integration of Sensible Cold Thermal Storage}

The first step was to determine how the cooling loads could be offset, as it is these loads that were responsible for the most significant portion of peak consumption within the house. A thermal storage tank was implemented into the house model, where the evaporator side of the chiller was connected to a sensible storage tank using a 50/50 water/glycol by volume solution as the storage medium. The model was run with the thermal storage tank with a volume of $270 \mathrm{~L}, 500 \mathrm{~L}$, and $1000 \mathrm{~L}$. The control of the heat pump was based on the average temperature of the tank, and the set-point was based on whether it was during peak or off-peak periods. During peak periods, the set-point was fixed at $17^{\circ} \mathrm{C}$ to limit the energy consumption during peak periods while meeting the cooling load of the house, while the set-point during off-peak periods was modelled at $5^{\circ} \mathrm{C}, 0^{\circ} \mathrm{C}$, and $-5^{\circ} \mathrm{C}$. These nine scenarios represent different total cooling potential stored, and the temperatures required to meet those storage capacities. Using $17^{\circ} \mathrm{C}$ as the reference temperature, as this is the temperature during peak periods in which the tank starts charging again, the total storage capacity and storage densities are tabulated in Table 6-6. 
Table 6-6: Storage densities for different volumes and set-points relative to $17^{\circ} \mathrm{C}$

\begin{tabular}{c|ccc}
\hline Volume (L) & $\begin{array}{l}\text { Off-Peak Set- } \\
\text { Point }\left({ }^{\circ} \mathrm{C}\right)\end{array}$ & $\begin{array}{l}\text { Storage } \\
\text { Capacity }(\mathrm{kWh})\end{array}$ & $\begin{array}{l}\text { Storage Density } \\
\left(\mathrm{kWh} / \mathrm{m}^{3}\right)\end{array}$ \\
\hline 270 & 5 & 3.27 & 12.14 \\
270 & 0 & 4.65 & 17.21 \\
270 & -5 & 6.01 & 22.27 \\
500 & 5 & 6.07 & 12.14 \\
500 & 0 & 8.60 & 17.21 \\
500 & -5 & 11.13 & 22.27 \\
1000 & 5 & 12.15 & 12.14 \\
1000 & 0 & 17.21 & 17.21 \\
1000 & -5 & 22.27 & 22.27 \\
\hline
\end{tabular}

The results for the simulations are presented in Figure 6-8, where the total consumption for peak, mid-peak and off-peak consumption is shown to meet the space heating and cooling demand (domestic hot water has been omitted as there is no impact and is constant for all scenarios).

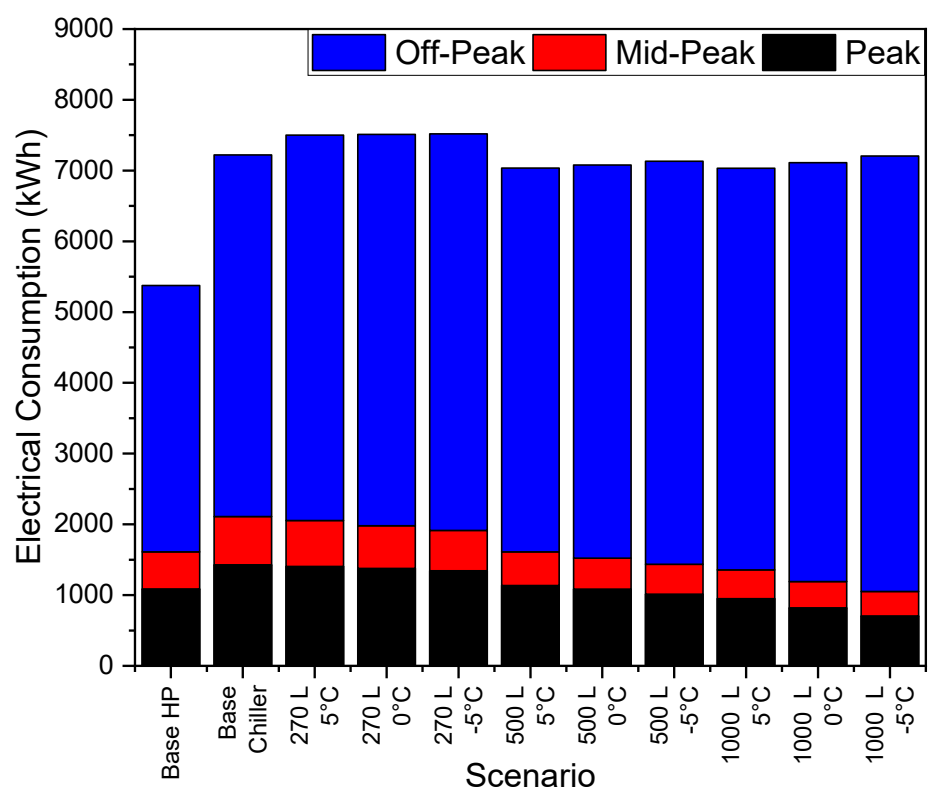

Figure 6-8: Annual electrical consumption for different cold storage volumes and set-points 
These results are further examined in Figure 6-9, where the total utility costs and greenhouse gas emissions are shown to meet the space heating and cooling demand for the year.

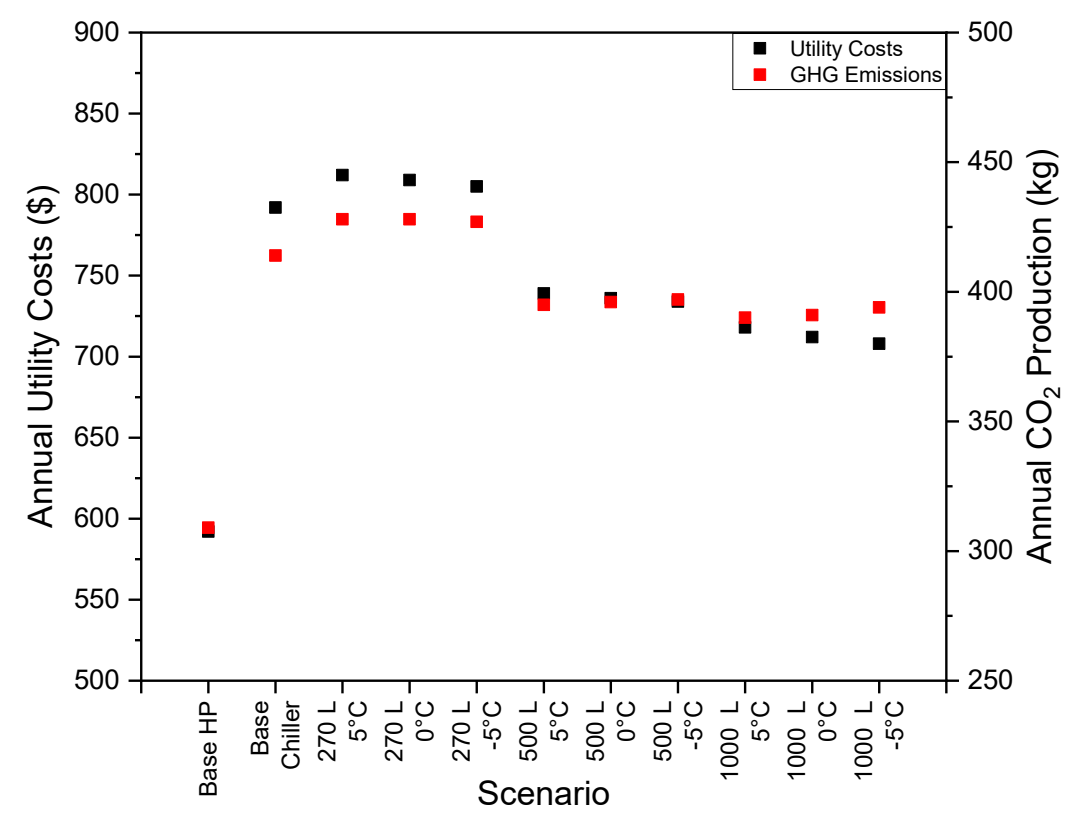

Figure 6-9: Annual utility costs and greenhouse gas production over different storage volumes and set-points

From these results, a number of conclusions were drawn. The first is that no matter the configuration, when using the medium temperature chiller it is impossible to meet the heating and cooling demands as efficiently as the standard heat pump, and in all scenarios modelled, a significant increase in overall consumption is observed. When looking at the integration of thermal storage into the system, a significant proportion of peak and midpeak loads can be shifted to off-peak periods, however a disproportionate increase in offpeak consumption is observed. This increase is partially offset by a reduction in off-peak heating during the shoulder seasons, where the waste heat generated through the charging of the cold thermal storage is used to simultaneously heat the space. This offset, due to the short period of time required to charge the tank in the shoulder seasons when both heating 
and cooling is permitted, does not make up for the decrease in the overall efficiency of the chiller to charge the thermal storage system when compared to cooling the space directly.

Although it was an important first step to look at the overall consumption on an annual basis, as most of the impact at this point is on the cooling, to determine the true impact of the different scenarios, just the energy required for cooling was examined in greater detail, and the annual consumption by time of use is shown in Figure 6-10.

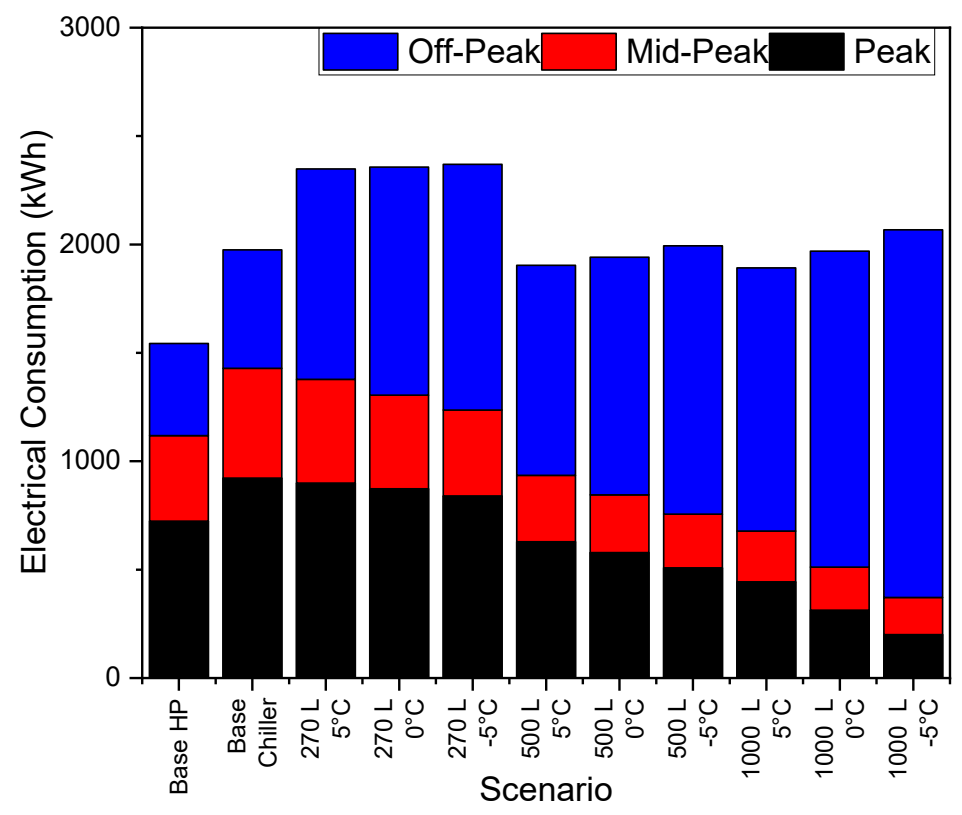

Figure 6-10: Electrical consumption to meet the space cooling demand only

It is also observed that decreases in costs are possible as more energy is shifted from peak to off-peak periods, however, significant volumes are required to off-set adequate peak consumption. Even with a $1000 \mathrm{~L}$ tank and a $-5^{\circ} \mathrm{C}$ average tank temperature set point, approximately two thirds of the peak and mid-peak energy is off-set. When the tank volume is decreased to $500 \mathrm{~L}$, which is the maximum realistic tank size that could be installed in a residence, only one third of peak and mid-peak cooling demand is offset. This showed that a thermal storage system with a higher storage density then a chilled glycol/water solution was required. 


\subsubsection{Integration of an Ice Storage Thermal Storage System}

Once the sensible cold thermal storage was tested and it was determined that the required tank volume was greater than what could be reasonably installed within a residential setting, particularly in retrofit situations where a tank of $1000 \mathrm{~L}$ or greater cannot be installed. As such, an ice storage system, which uses the latent heat of formation to store cooling potential with a much greater storage density then sensible storage. A $500 \mathrm{~L}$ tank was selected and replaced the sensible storage tank in the house model. A total of 144 ice formation pipes were modelled inside the tank. A control strategy was implemented, where the amount of cooling potential stored was used as the control variable, and two set-points, one for during off-peak periods and one for peak periods, were implemented. Through all tests, a peak storage capacity of $4 \mathrm{kWh}$ was implemented, while the off-peak storage capacity set-point was left variable to determine the optimal set-point for the system. A parametric study with the off-peak, stored cooling potential set-point was conducted, varying the set-point from $10 \mathrm{kWh}$ to $35 \mathrm{kWh}$ (slightly above $50 \%$ ice formation) in $5 \mathrm{kWh}$ increments. The results of this study when looking at the total annual utility costs are shown in Figure 6-11. 


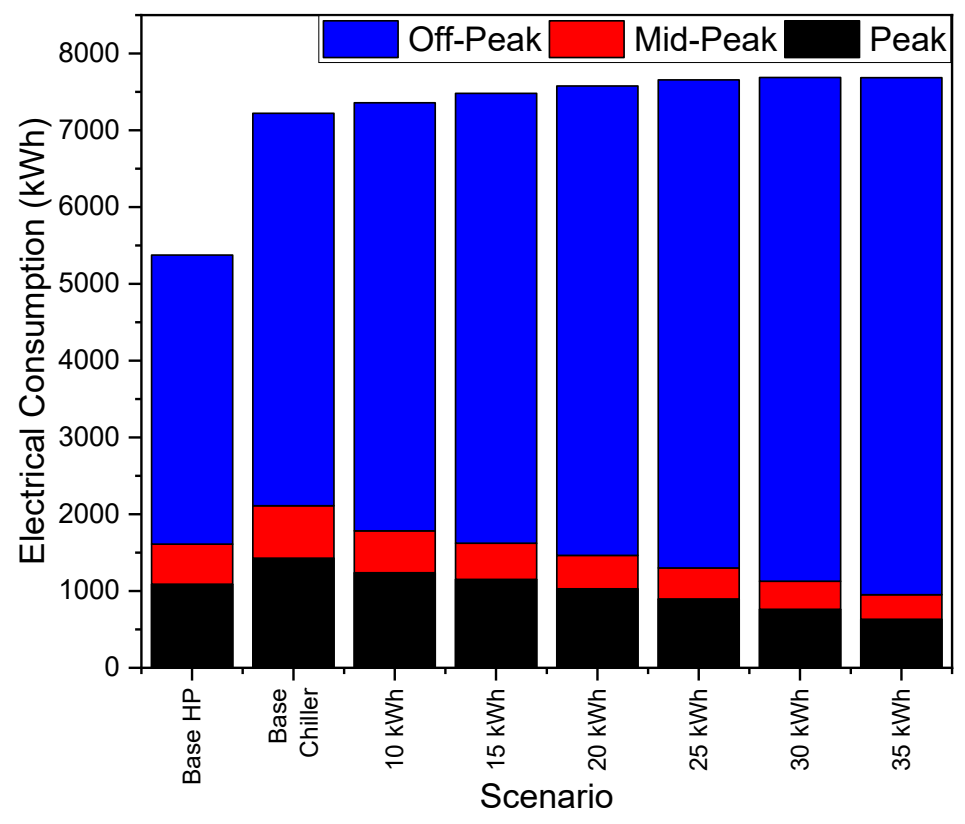

Figure 6-11: Electrical consumption using an ice storage system with different set-points

These results are very similar to those using the sensible storage tank, and again, the impact of the cold thermal storage is drowned out by the large percent of annual heating load. As such, just the cooling was examined for the same scenarios, and these results are shown in Figure 6-12.

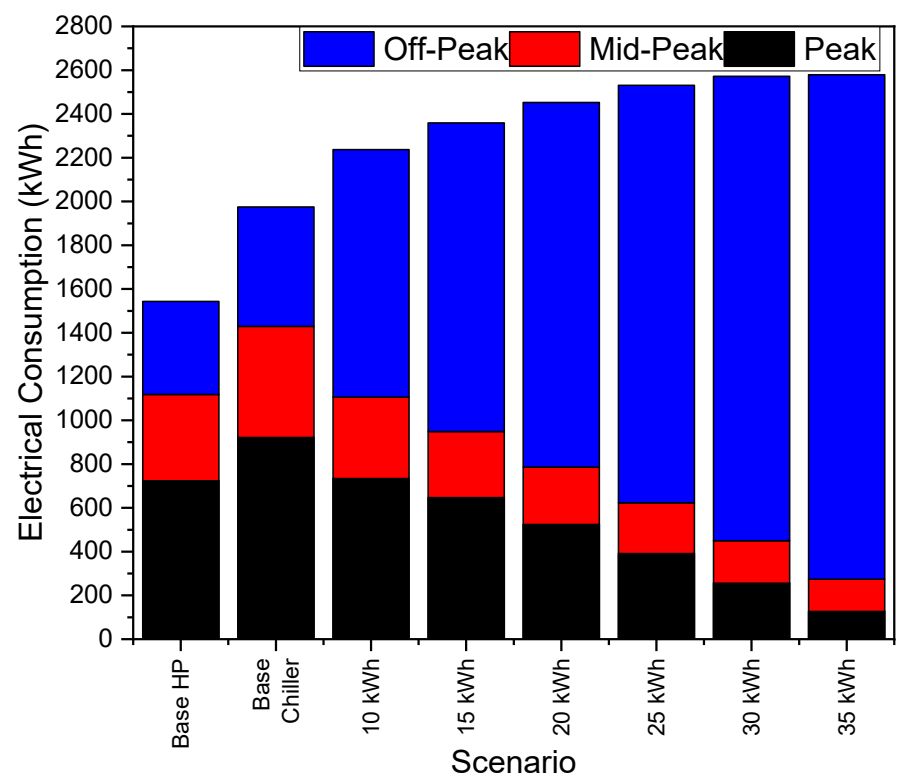

Figure 6-12: Electrical consumption to meet cooling demand using an ice storage system with different set-points 
These results show that the increased storage capacity possible with the ice storage when compared to the sensible storage allow for a greater percentage of the peak loading to be off-set, with only $10 \%$ of the cooling load during peak periods (compared to $72 \%$ in the base case). When the annual utility costs and greenhouse gas production is factored in, the system still creates a significant increase in the total utility costs and greenhouse gas production, as shown in Figure 6-13.

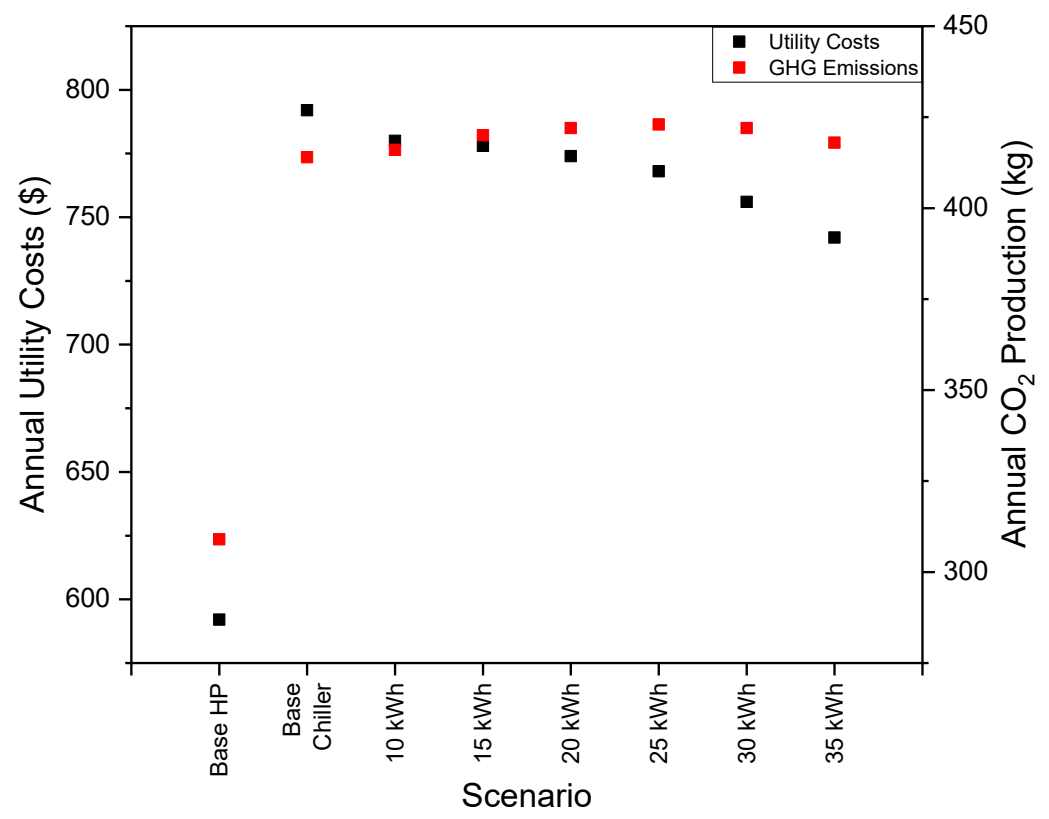

Figure 6-13: Annual cost and greenhouse gas production with an ice storage system and different setpoints

Based on these results, it was determined that in Ottawa, with the cooling loads a typical house would experience, and the ratio of the cost of electricity between peak and off-peak periods, offsetting peak cooling loads alone would not produce an annual utility savings. As such, further design changes need to be explored to determine whether there is 
the potential for achieving cost and greenhouse gas reduction through the shifting of residential peak loads.

\subsection{Integration of Domestic Hot Water Loads}

To increase the amount of peak energy consumption off-set through the system, either the DHW or heating loads needed to be incorporated into the system. When looking at the base loads, $60 \%$ of the DHW load occurred during peak periods, while only $13 \%$ of the heating load occurred during peak periods. As such, the offsetting of electrical peak loads to meet the DHW load of the system was further examined.

The base model utilized an on-demand, electric hot water heater to meet the DHW demand. A profile developed by the Canadian Standards Association [82] was utilized in the model, where a total of $225 \mathrm{~L}$ of hot water was consumed daily, which represents the typical demand for a family of four. This profile provides an hourly profile provided, in which the greatest demand occurs early in the morning and in the early evening. Both of these periods occur during peak/mid-peak periods and as such, represent a significant potential for reducing the peak electrical consumption. To begin, a $270 \mathrm{~L}$ standard hot water tank with no internal heating coils was implemented into the model. This tank was connected to the hot side of the chiller, and a control strategy was implemented using the average tank temperature as the control value. The chiller would not operate to simply charge the DHW tank, but when the chiller is running to meet the cooling load or to charge the cold thermal storage, and the DHW tank is below the chosen setpoint, the waste heat from the chiller is diverted to the DHW tank. If the DHW tank has reached its set-point, the chiller will resume its normal operation, where it dissipates its waste heat outdoors.

This system is not designed to meet $100 \%$ of the DHW load, but to preheat the 
water before it enters the on-demand heater which will heat the water up to $55^{\circ} \mathrm{C}$ as prescribed by the CSA standard. As such, the only variable that changes in the system, if it is assumed that the volume of the tank is constant, is the set-point for the DHW tank to charge. The higher the set-point temperature, the greater the percentage of hot water demand met through this system, but at a cost of decreased chiller performance. As such, a parametric study was conducted to determine the impact of the control variable on the overall system performance and was varied from $30^{\circ} \mathrm{C}$ to $55^{\circ} \mathrm{C}$ in $5^{\circ} \mathrm{C}$ increments. A final test was conducted with a set-point of $50^{\circ} \mathrm{C}$, but with the tank volume increased to $375 \mathrm{~L}$, to represent a larger, typically utilized, domestic hot water tank. The results showing the energy consumption, utility costs and greenhouse gas production when considering the space heating, cooling and domestic hot water demand are shown in Figure 6-14 and Figure 6-15.

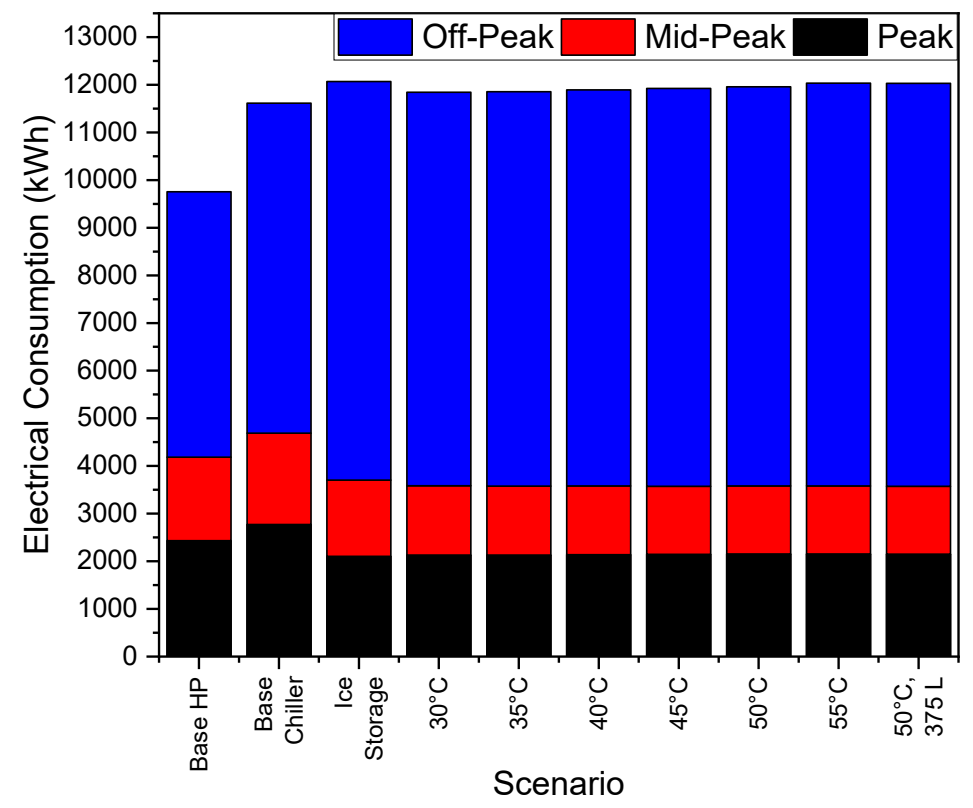

Figure 6-14: Annual electricity consumption to meet space heating, cooling, and DHW loads Integrated DHW 


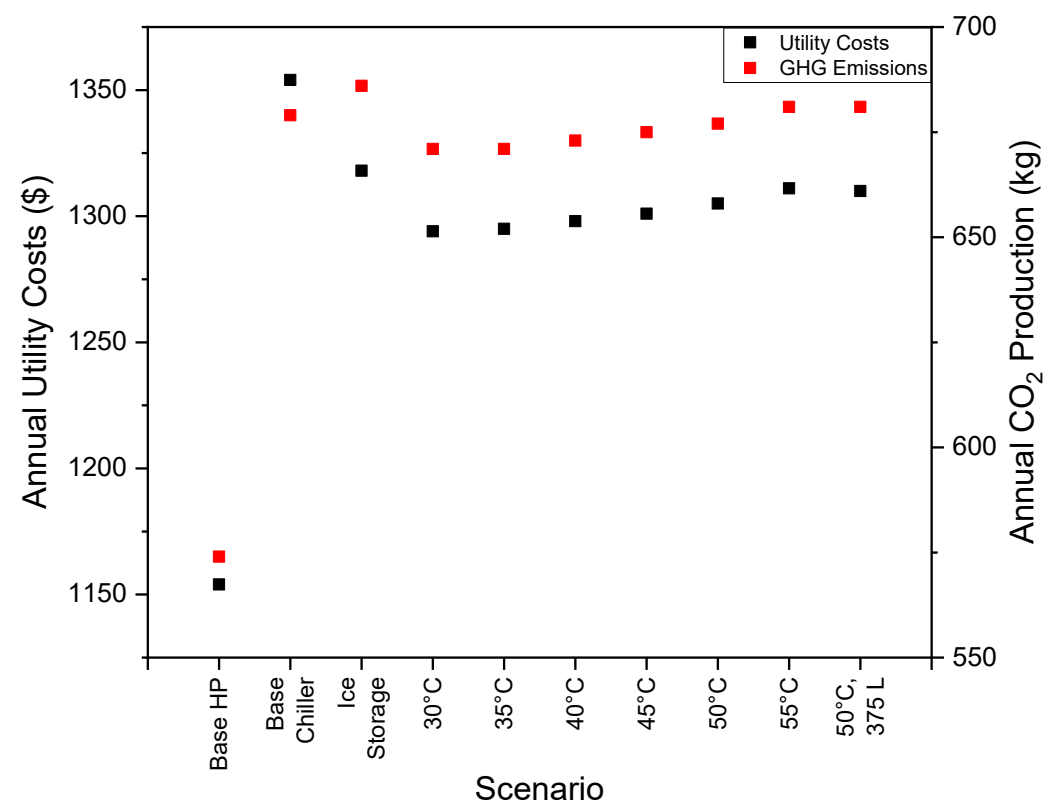

Figure 6-15: Annual utility costs and greenhouse gas emissions - Integrated DHW

As the integration of the domestic hot water has minimal impact on the heating load of the building, the energy required to meet the space heating demand was removed and the results were replotted to get a better gauge of the impact on the required energy to meet the building's requirements, and are shown in Figure 6-16. 


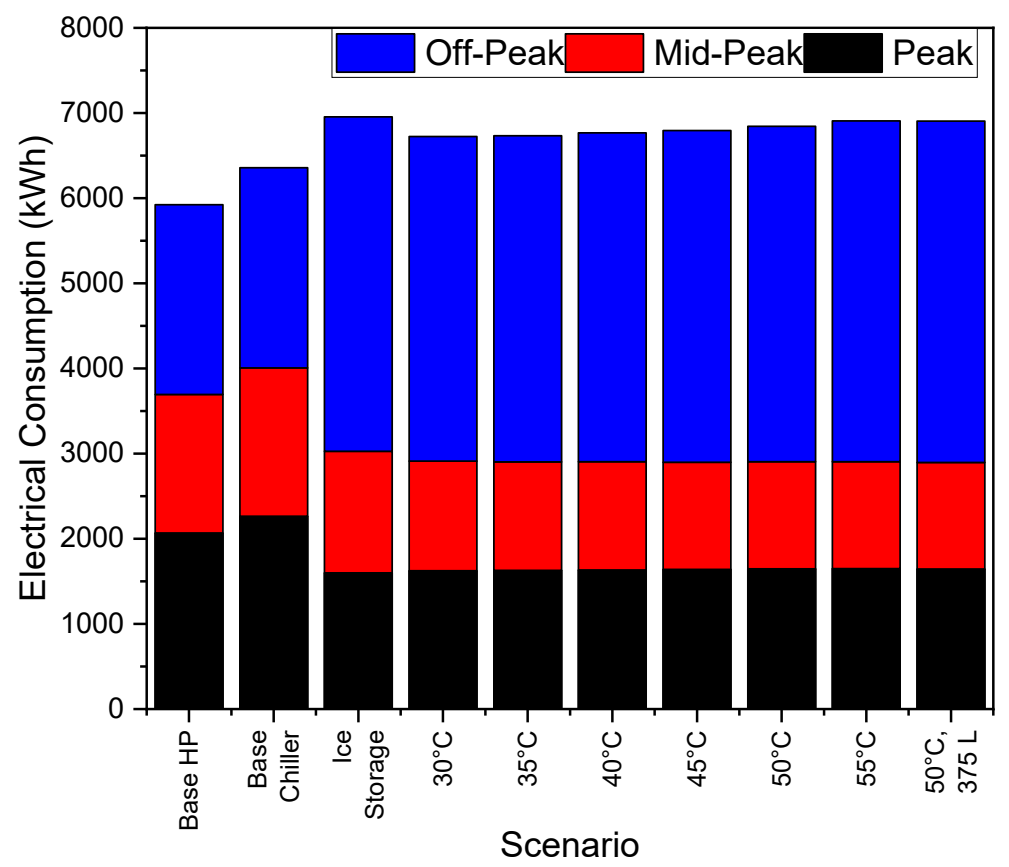

Figure 6-16: Electrical consumption to the meet the space cooling and DHW demands of the building

Based on both the total results incorporating the heating load, and those in which the heating consumption is omitted, a decrease in total consumption, peak/mid-peak consumption and annual utility costs are realized when the DHW tank is integrated with a low set-point temperature. As the set-point increases for the tank, the return temperature to the condenser increases, causing a significant degradation in chiller performance. This decrease in performance is more significant than the benefits that can be derived from using the waste heat from chiller as a "free" source of energy to meet the DHW demand. As such, it was decided that the integration of the DHW tank would run with a set-point of $30^{\circ} \mathrm{C}$.

A separate test was run at to determine the impact of a slightly larger tank volume, in which a slight increase in performance of the system as a whole was observed, but a temperature set-point of $50^{\circ} \mathrm{C}$ was utilized. As it was shown that the $30^{\circ} \mathrm{C}$ set-point was optimal for energy and cost savings, a second set of simulations were run with a $30^{\circ} \mathrm{C}$ setpoint, but with tank sizes of $150 \mathrm{~L}, 225 \mathrm{~L}, 300 \mathrm{~L}$, and $375 \mathrm{~L}$, with the results for just 
cooling and DHW loads shown in Figure 6-17, while the annual utility costs are shown in Figure 6-18.

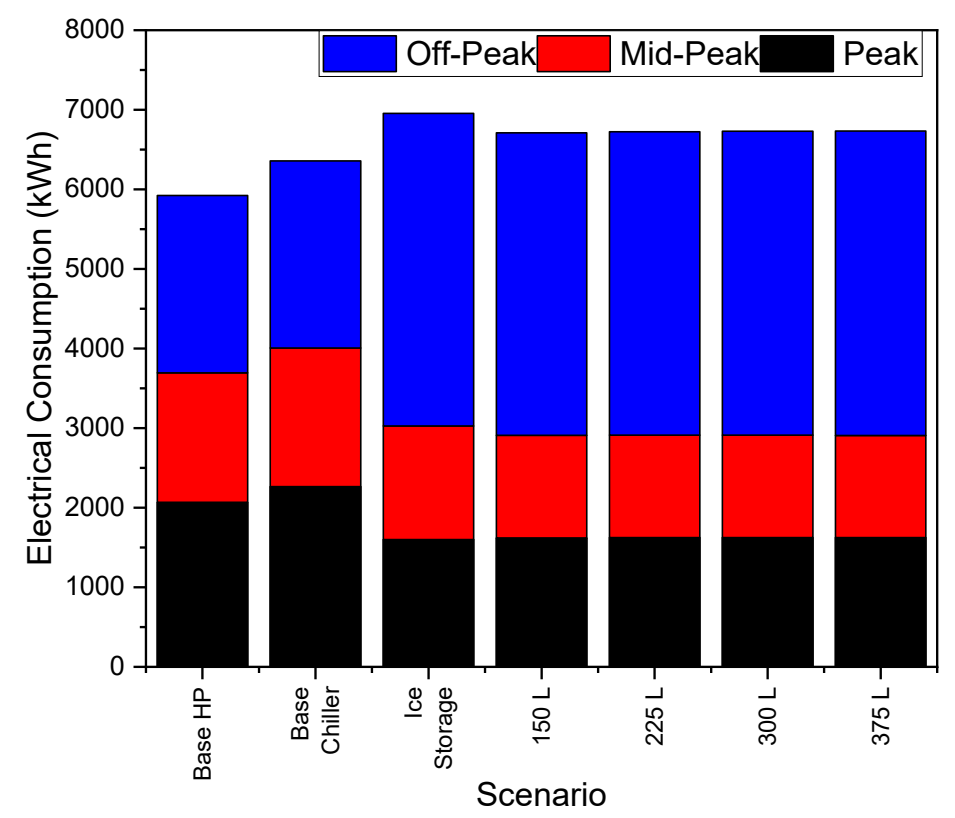

Figure 6-17: Impact of pre-heat tank volume on annual consumption for space cooling and DHW

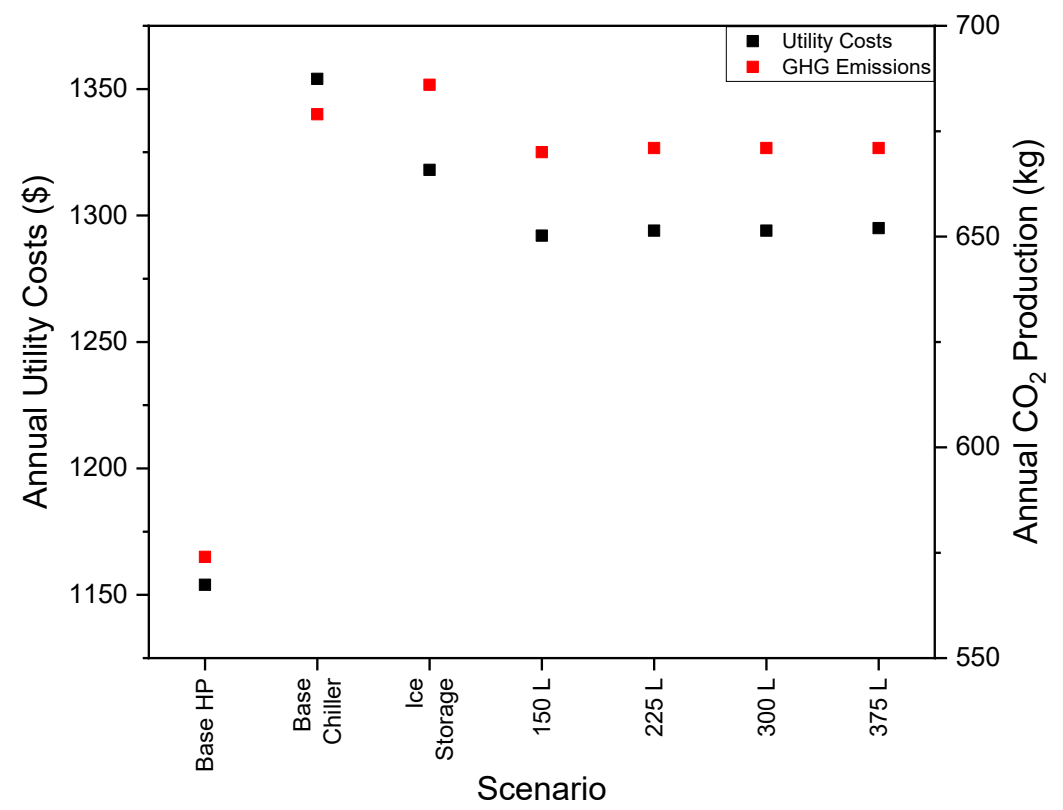

Figure 6-18: Impact of pre-heat tank volume on annual utility costs

These results reiterate that a reduction in total energy use and utility costs are realized by the integration of a DHW pre-heat tank with a set-point of $30^{\circ} \mathrm{C}$. The results 
also show that there is basically no change in overall performance between the different tank volumes, and actually a small increase in utility costs as the tank volume is increased. This is caused by a longer period of time where the chiller must operate at higher condenser temperatures, and the energy within the pre-heat tank is not utilized as the volume of the tank is greater than the volume of water supplied to the residence to meet the demands of the occupants. Based on these results, and the overall system being designed to offset peak and mid-peak loads, a preheat tank with a volume of $150 \mathrm{~L}$ is integrated and utilized within the system.

Although this system was only able to offset a small portion of the peak and midpeak DHW loads, it was able to decrease the overall energy consumption required to meet

all the building loads. A completely separate and stand-alone system could be integrated into the building, only to offset the DHW loads, most likely using a large water tank and electric resistance heater or a heat pump water heater with a control strategy that has a much higher set-point during off-peak periods, when compared to the peak periods. Although this would move consumption to off-peak periods, it falls outside the goal of this project which is the integration of a heat pump/chiller with thermal storage systems to move peak and mid-peak consumption to off-peak periods.

\subsection{Integration of Heating Loads}

The final building load that had to be examined to determine the potential for offsetting peak and mid-peak loads is the space heating. The space heating load had the smallest proportion of its load during peak and mid-peak times, as the solar heat gains through the building are greatest during the peak and mid-peak periods, and the outdoor temperatures are coldest during off peak periods. These factors combine to create more 
demand for space heating during overnight periods, but there is still overall a significant amount of energy required for space heating, particularly in northern locations like Ottawa. As such, one final thermal storage system was added, using $500 \mathrm{~L}$ of water for sensible storage, and connected to the heating coils of the house.

This system was controlled with two separate control set-points which monitored the average tank temperature, one at a higher temperature during peak periods, allowing energy not being used to meet the space heating loads to be stored, and a lower set-point during peak periods, that will maintain adequate temperature to meet the space heating loads, but will not store additional energy. The set-point is fixed during peak periods at $28^{\circ} \mathrm{C}$, while a modelling study was conducted to determine the impact of the set-point during off-peak periods on the total energy consumption, as well as the amount of energy utilized during peak periods, and consequently the impact on utility costs and greenhouse gas emissions. These results, for the system incorporating the ice storage system with a setpoint of $35 \mathrm{kWh}$, the $150 \mathrm{~L}$ DHW pre-heat tank set to $30^{\circ} \mathrm{C}$ and the newly integrated hot storage tank with a volume of 500 L, are shown in Figure 6-19 and Figure 6-20. 


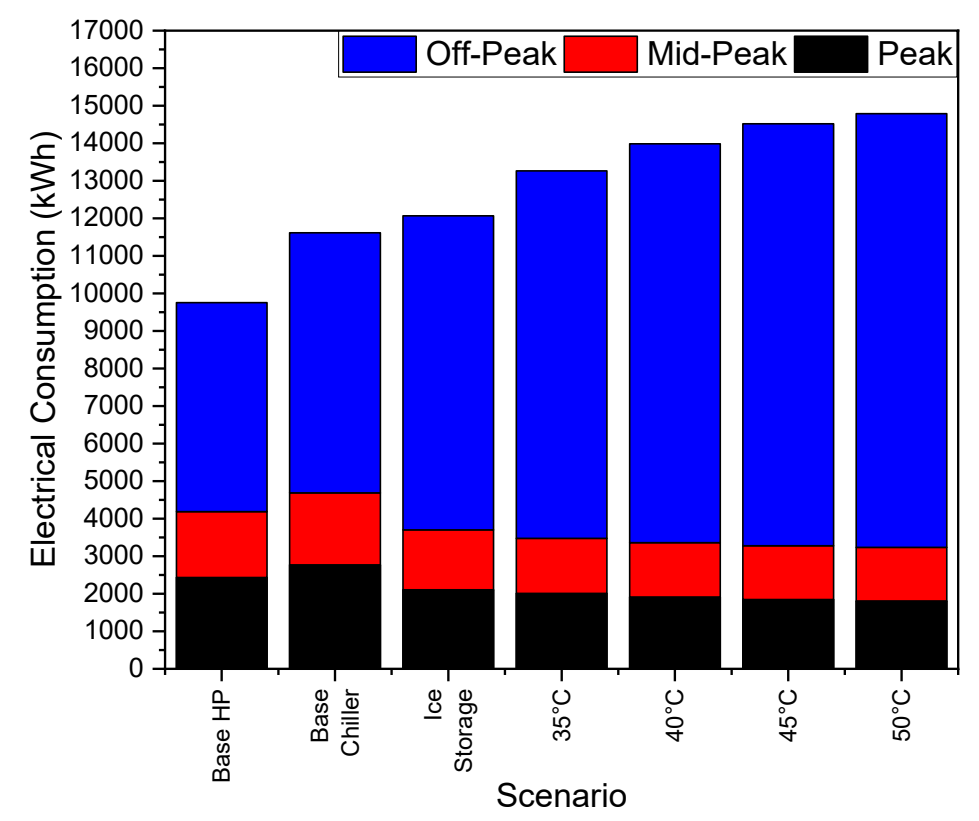

Figure 6-19: Annual energy consumption when a sensible hot storage system is integrated

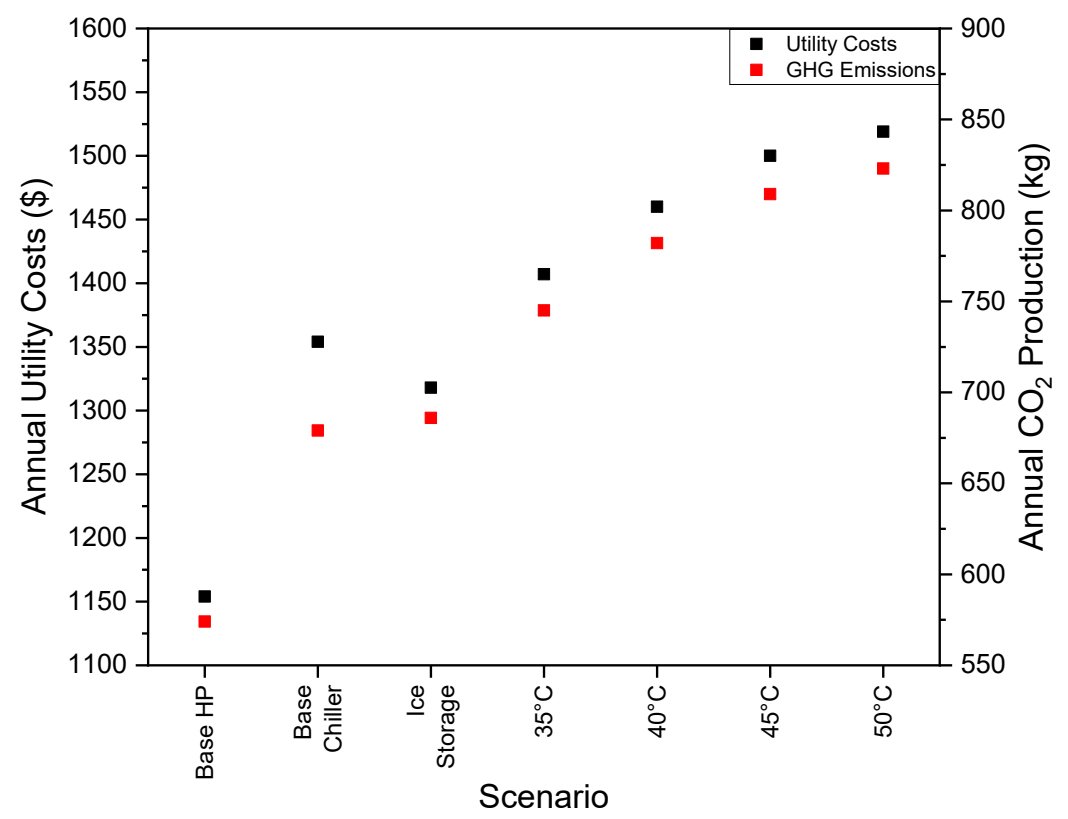

Figure 6-20: Annual utility costs and greenhouse gas emissions using sensible hot storage

These results show that when working to off-set the peak load, a significant increase is observed in the total consumption, annual utility costs, and greenhouse gas emissions. When observing the off-set energy, it was determined that at the $35^{\circ} \mathrm{C}$ set-point, each unit of peak energy off-set required an additional 6.2 units of peak energy, with only $230 \mathrm{kWh}$ 
of peak energy being offset. This increased to over 6.8 unit of energy for each unit of peak energy offset, while off-setting $468 \mathrm{kWh}$ when a set-point of $50^{\circ} \mathrm{C}$ was used. This is compared to an increase of $\sim 1.5$ units of off-peak energy for every unit of energy off-set when compared to using the medium temperature chiller to directly meet the space heating and cooling.

Based on these results, it is not feasible to directly use a sensible hot thermal storage system to off-set peak loads when directly controlling the amount of energy stored by using a fixed value for the thermal storage on an annual basis. One of the challenges is on day where little heating or cooling is required, the stored energy or cooling potential must be replenished under the worst potential conditions, where the heat pump/chiller operate with the lowest COPs. In other words, the first energy consumed from the storage tank is the hardest energy to replace. As such, the potential for determining how much energy is required for a given day was examined and discussed in the proceeding section, as a method for improving the efficiency at which energy can be stored and utilized for both heating and cooling applications.

\subsection{Utilization of Predictive Controls for Determining Storage Set-Points}

Once the impact of adding thermal storage systems to the cooling, DHW and heating system was assessed, the final component that was examined in detail to determine the potential for demand side management in Ottawa is the control strategies utilized. In all cases examined to this point, static control strategies were utilized, in which the amount of energy stored in each of the thermal storages (through a proxy being the average tank temperature in some cases), were used to control the heat pump. As previously discussed, this strategy is the simplest method to control the systems, but results in an increase in 
energy consumption as the energy that requires the greatest energy input to store is always being used first, and the total energy stored is rarely fully utilized. As such, alternate control strategies are examined to determine whether alternative strategies would allow for increased annual performance.

\subsubsection{Determining Relationship Between Weather Conditions and Loads}

The key to reducing the amount of energy required to shift consumption from peak to off-peak periods, is to predict the amount of energy that needs to be stored to meet the peak demand, and only store that amount of energy for a given day. To achieve this, a predictive control strategy must be utilized, taking in some number of variables or prediction of variables, and determining how much heating or cooling should be stored to meet that load. Although a number of factors contribute to the required heating and cooling loads of a building, they are predominantly driven by two main factors: the outdoor temperature and the solar radiation incident on the building. To develop a predictive control system, it is necessary to predict the value of one of these variables and relate that to the peak energy consumption. As weather forecasts are readily available for anywhere from 1 to 7 days in advance, a relationship between the daily high temperature and the peak heating and cooling load was explored. The maximum daily high was selected as the prediction variable, as all weather forecasts include this value, while solar radiation or sun conditions are not readily available. To determine whether a relationship can be derived, a simulation was run where the total peak heating and cooling demand are compared to the maximum temperature for the day. These results are for heating and cooling loads are graphed in Figure 6-21. 

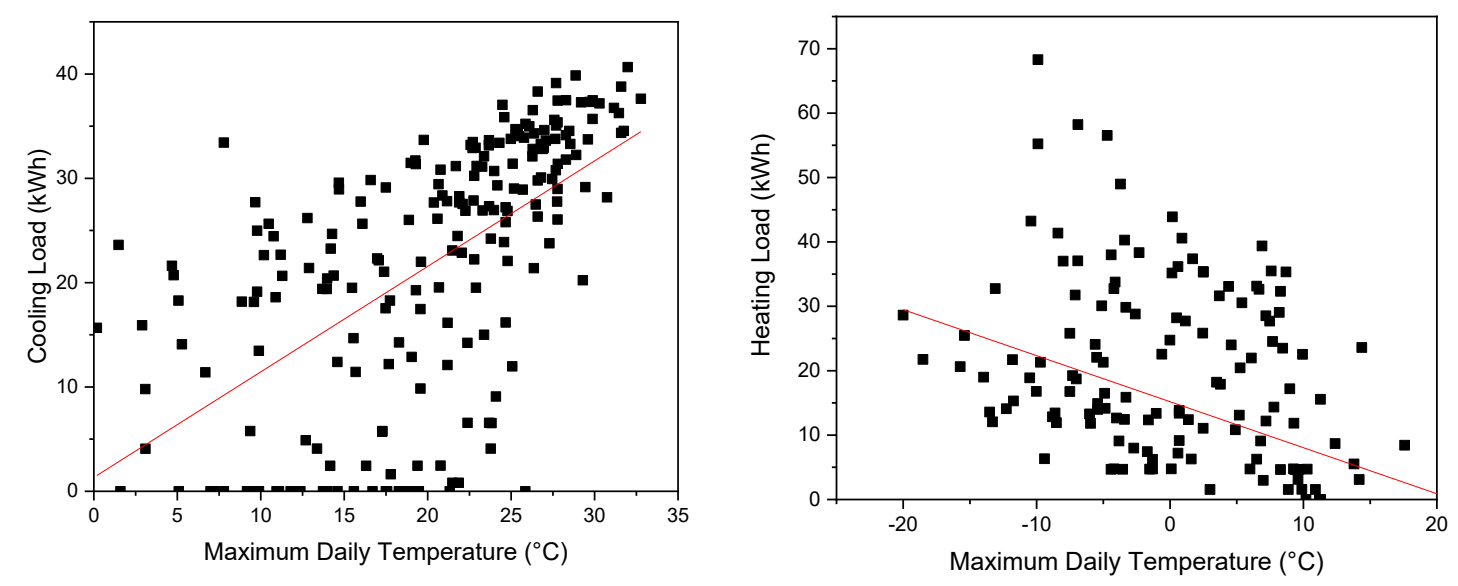

Figure 6-21: Plot of daily maximum temperature and cooling load(left), and heating load (right)

A linear trendline was applied to both graphs and an equation was determined that related the daily peak heating and cooling load to the maximum temperature, and are provided in Equation (6-1) and (6-2), along with their $\mathrm{R}^{2}$ values.

$$
\begin{array}{ll}
\text { Peak_Cooling_Load }=1.01\left(T_{\mathrm{Max}}\right)+1.33 & \mathrm{R}^{2}=0.35 \\
\text { Peak_Heating_Load }=-0.627\left(T_{\mathrm{Max}}\right)+17.53 & \mathrm{R}^{2}=0.23
\end{array}
$$

First examining the relationship for the cooling, the $\mathrm{R}^{2}$ value is relatively low, but most of the large deviations from the trendline occur at the lower temperatures, when the overall cooling required is at its lowest. This shows it is much easier to predict the cooling load on hot days, as opposed to cooler days where there could the solar gains would have a larger impact on the required cooling. Having the deviations at the lower cooling loads is preferred as these will have a much smaller impact on the overall performance of the system, and the annual performance of the system. Proceeding with the derived trendline, this represents the average relationship between the peak loads and the daily temperature. If this was used as the daily cooling load set-point then, during approximately $50 \%$ of the days, the amount of cooling potential stored would not allow all of the peak load to be offset. As such, it was decided that a fixed value would be added for each the calculated 
cooling load set-point, and values of $5 \mathrm{kWh}, 10 \mathrm{kWh}$, and $15 \mathrm{kWh}$ were plotted on the original graph, as shown in Figure 6-22, to determine the impact on encompassing the greatest number of days, while reducing the potential for, and magnitude of overcharging the thermal storage system.

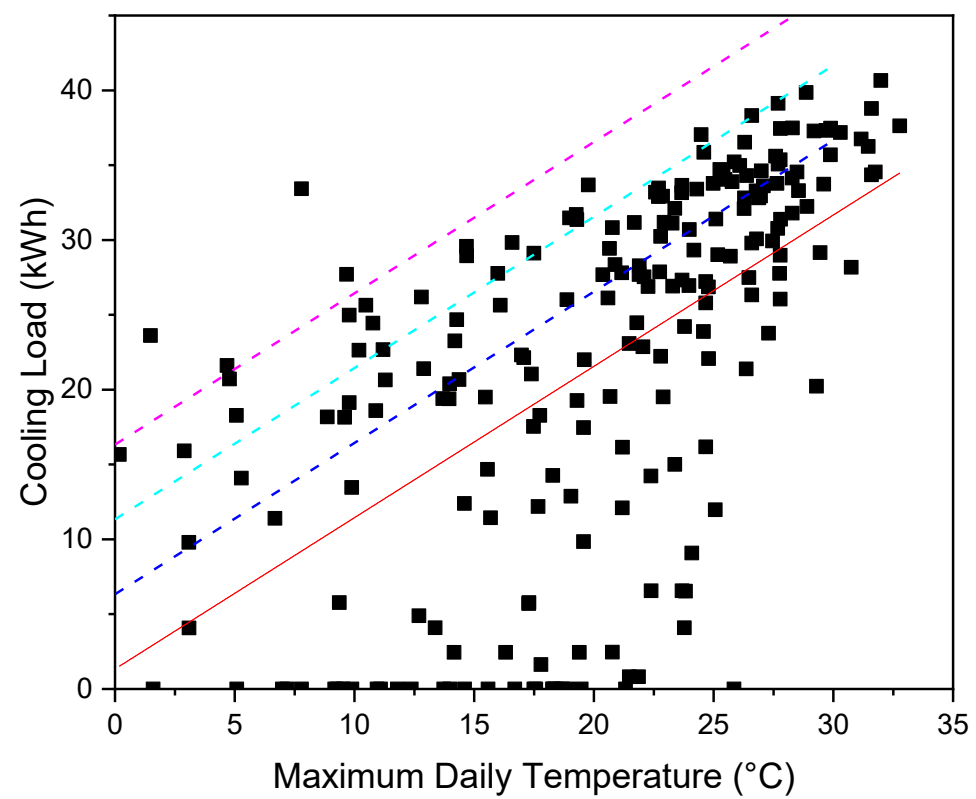

Figure 6-22: Cooling load compared to daily high temperature, with steps added to trendline

Based on this plot, a step of $10 \mathrm{kWh}$ was selected and added onto Equation (6-1), as at this point, the set-point for the cold storage encompasses the required cooling load for each day. This also limited the number of days in which the set-point would store more cooling then required, compared to the $15 \mathrm{kWh}$ step, and therefore will store cooling at the highest efficiency possible. The new equation, used within the simulations to determine the daily set-point for the amount of cooling to store in $\mathrm{kWh}$ is shown in Equation (6-3).

$$
\text { Cooling }=1.01\left(T_{\operatorname{Max}}\right)+11.33
$$

After determining the equation that will be used to test this proposed control strategy, the relationship between the maximum daily temperature in degrees Celcius and 
peak loads was further examined. This had an even lower $\mathrm{R}^{2}$ value compared to the cooling, and the difference between the trendline and actual values increased as temperature decreased and heating loads increased. In this case, the discrepancies observed would have a significant impact on the performance of the system, so alternative options for predicting the peak heating load were further explored. A second variable, commonly available in weather forecasts is the daily low, and as low temperatures are the leading driver of heating loads, these values were plotted to determine if a relationship can be derived, as shown in Figure 6-23.

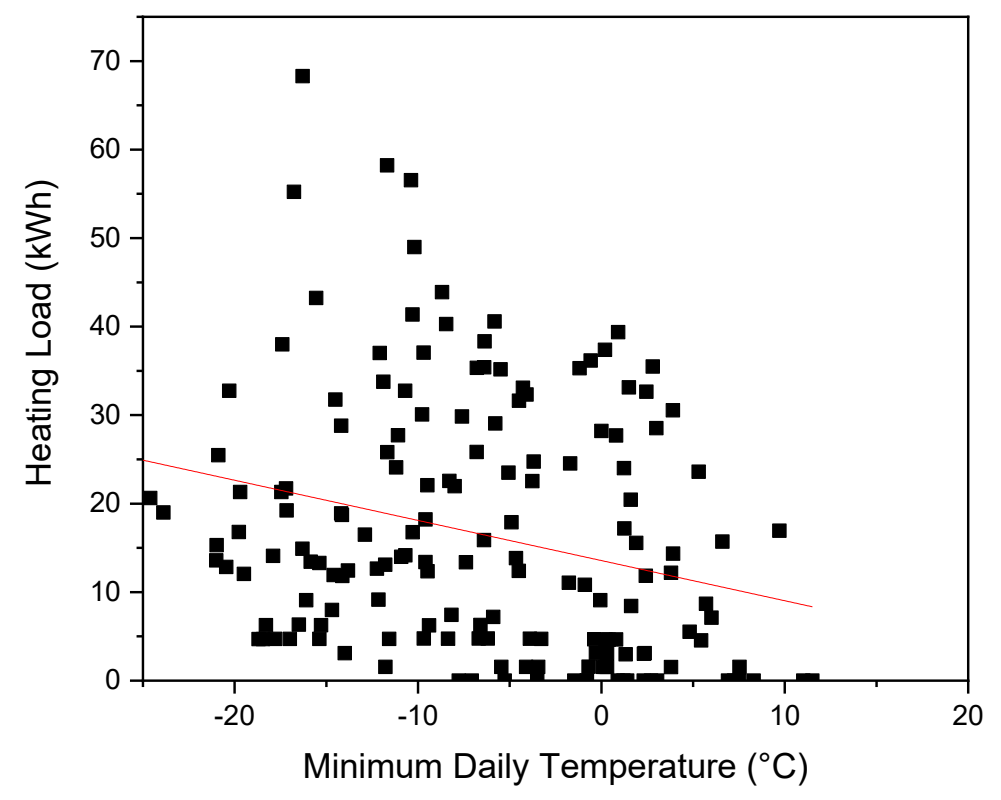

Figure 6-23: Relationship between heating load and daily minimum temperature

It was immediately obvious that there was not relationship with between the daily low temperature and amount of heating required during peak period. The trendline for this "relationship" was found to have an $\mathrm{R}^{2}$ value of only 0.06 , so the idea of switching to 
predictive variable being the daily low was abandoned and the results for using the daily maximum temperature was further evaluated.

Because the performance found for the system as a whole degraded quickly as the set-point temperature was increased, the amount of heating energy that can be offset was further examined. If the $35^{\circ} \mathrm{C}$ off-peak was utilized, and $24^{\circ} \mathrm{C}$ as the reference temperature (minimum temperature possible for space heating) and a $500 \mathrm{~L}$ tank volume, a total of $6.4 \mathrm{kWh}$ of heat could be stored. This increases to $12.2 \mathrm{kWh}$ at a set-point of $45^{\circ} \mathrm{C}$. When looking at the required heating to offset the peak loads, based on the trendline, $12.2 \mathrm{kWh}$ hours of storage equates to a maximum temperature of $8.5^{\circ} \mathrm{C}$. During the heating season, this equates to very few days in which a predictive control would alter the amount of energy stored, with the current system design and as such, predictive control was only implemented to control the cold storage, based on its limited effectiveness for predicting the required heating demand that should be stored.

\subsubsection{Modelling Predictive Control}

Once the relationship between the peak cooling load and the maximum daily temperature was determined, the next step was to develop a methodology for determining

the forecasted daily high. A report by Rose and Floehr [83] examined the accuracy of forecasted daily highs one, three, and seven days in advance. As the control is implemented one day in advance, only their conclusions on the accuracy of 1-day out forecasts were considered. They determined that weather forecasts were accurate based on the rates found 
in Table 6-7. Using these values, and the probabilities of a normal distribution, the standard deviation for each range was determined.

Table 6-7: Accuracy of weather forecasts

\begin{tabular}{l|cc}
\hline $\begin{array}{l}\text { Accuracy of } \\
\text { Forecast }\left({ }^{\circ} \mathrm{C}\right)\end{array}$ & $\begin{array}{c}\text { Percentage of } \\
\text { Occurrence } \\
(\%)\end{array}$ & $\begin{array}{c}\text { Standard } \\
\text { Deviation }-\sigma \\
\left({ }^{\circ} \mathrm{C}\right)\end{array}$ \\
\hline \pm 0.55 & 15.7 & 2.28 \\
\pm 1.67 & 79.4 & 1.32 \\
$>5.55$ & 1.5 & 2.41 \\
\hline
\end{tabular}

Within the model, a CWEC weather files were utilized to represent the environmental conditions that the building experienced. As the forecast for each day utilized in the weather year are not available, a "forecasted" temperature for each day of the year had to be determined based on the available weather file. The daily highs for each day were previously determined and were used to develop a set of forecasted daily highs using Equation (6-4).

$$
T_{\mathrm{F}}=T_{\max }+T_{\text {random }}
$$

where $T_{\mathrm{F}}$ is the calculated, forecasted daily high temperature, $T_{\max }$ is the maximum temperature seen on the particular day in the weather file for the corresponding city, and $T_{\text {random }}$ is value the temperature forecast is off based off of a randomly generated number, using the maximum standard deviation found in Table 6-7 and a mean value of 0 .

Using this formula, a forecasted high for every day of the year is determined. To ensure that any given forecasted year isn't overly bias in one direction, a second set of random numbers were determined. Using these values, a second forecasted year was developed for Ottawa. As a comparison of the two forecasts and the actual daily high temperatures, Figure 6-24 shows the data for the month of July. 


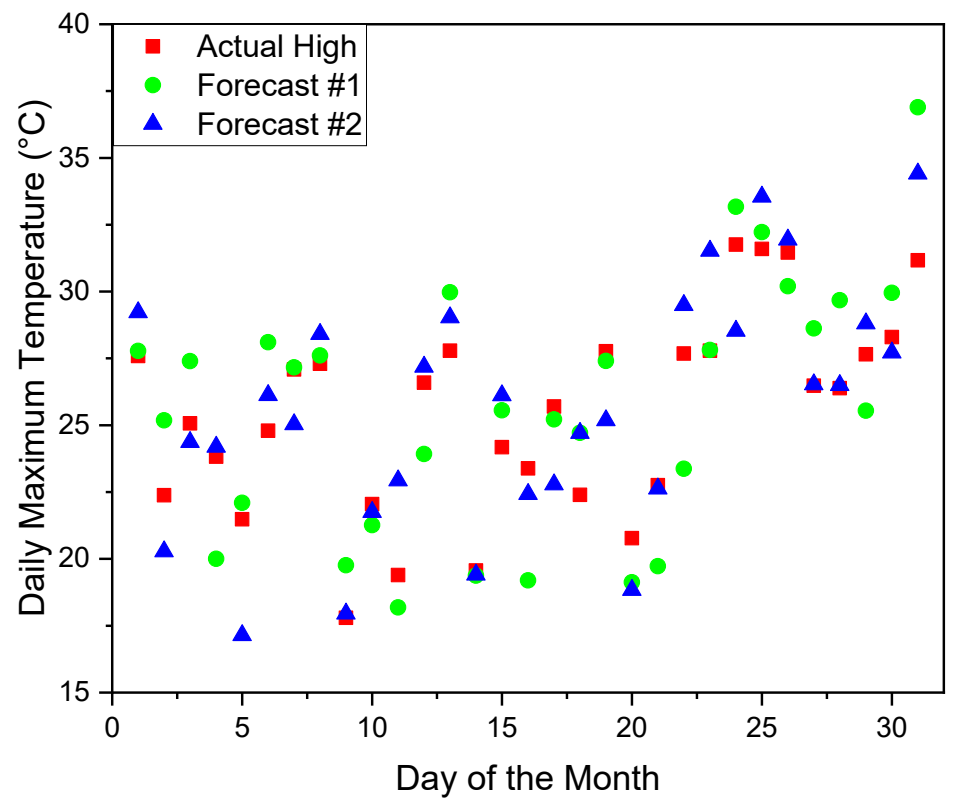

Figure 6-24: Comparison of actual and forecasted daily highs for the month of July

These forecasts were then implemented into the control system of the modelled system. At midnight, the forecasted high for that day is read into the control system, and using the equation (6-3), the predicted peak cooling load for the next day was then determined.

In addition to the set-point calculated based on the weather, a second static set-point is set, which is the minimum lowest set-point allowed based on the capacity for the ice storage, and as previously determined for this system set-up, is $30 \mathrm{kWh}$ of cooling potential. Once the two values were determined, they were then compared and the higher of the two values was taken as the set-point for the day. The system then charged the tank to this set-point, and once charged, continued to maintain that charge until the off-peak period ended and the peak period began.

If, during peak periods, the tank exhausts all of its cooling potential, then a secondary control takes over and maintains the tank with a minimum of $4 \mathrm{kWh}$ of stored cooling potential. Once the peak period is over, the tank control remains with the secondary 
control scheme until midnight, when the set-point for the next day is determined and the control cycle starts again.

\subsubsection{Results using the Predictive Control Strategy for Storing Cooling}

The previously discussed modelling method and control strategy were implemented into the model, where the DHW pre-heat tank was incorporated, but without any hot thermal storage. This was done to better see the impact on the required electrical input to meet the cooling and DHW loads, which this control strategy would impact. The annual energy consumption to meet these loads using the forecasted control strategy are shown in Figure 6-25, while the annual utility costs and greenhouse gases are shown in Figure 6-26.

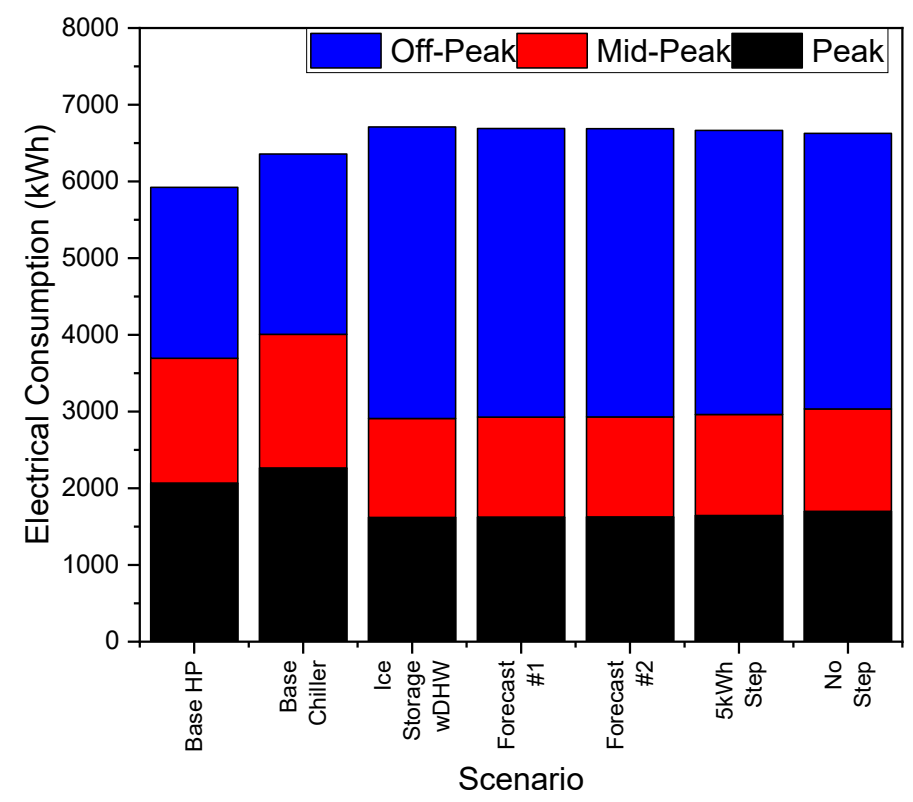

Figure 6-25: Annual consumption using a predictive control system 


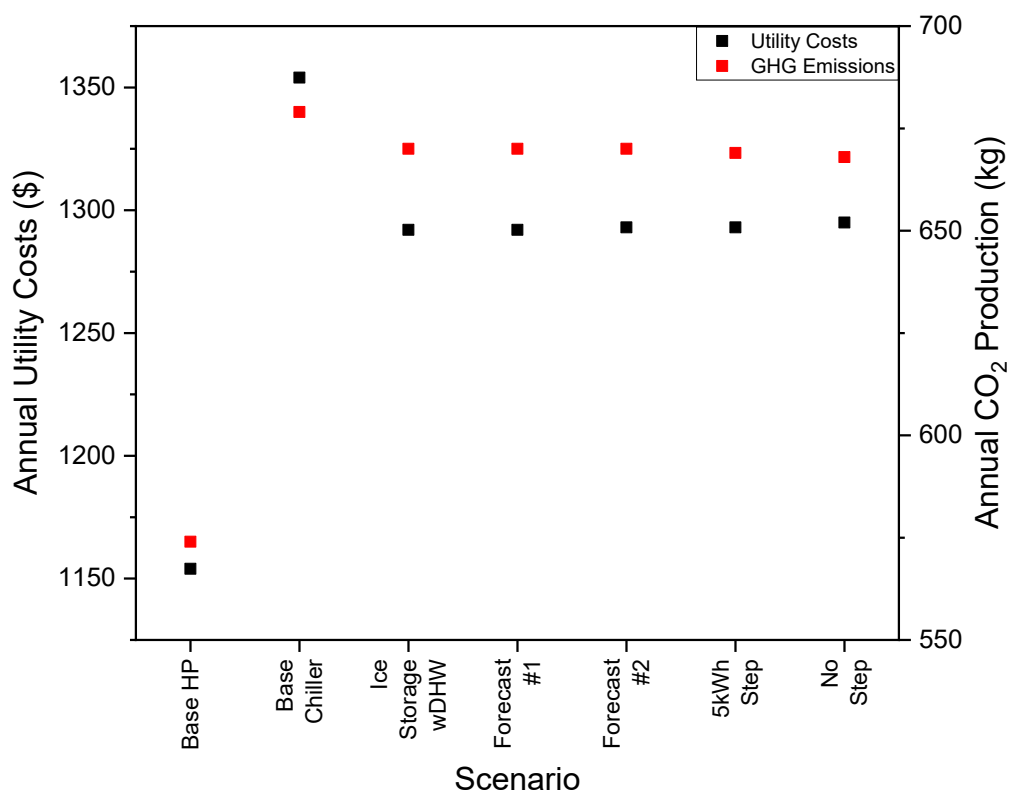

Figure 6-26: Electrical costs and greenhouse gas production using a predictive control system

When comparing the two forecast controls, both forecasts created very little change between the two modelled years. This showed that there is minimal impact created by the randomly generated in forecasts. When the results based on the originally derived equations, which included a $10 \mathrm{kWh}$ increase from the trendline, there was basically no change between the forecast control and the original static control. There was a total decrease in energy consumption of $19 \mathrm{kWh}$ for the cooling and DHW loads, while the peak electrical consumption increased by $18 \mathrm{kWh}$. This was a result of the fact that with the 10 $\mathrm{kWh}$ step implemented, the peak load is offset basically every day, and there is little change with this off-set when compared to the static control strategy. As such, two additional annual simulations were run, first where that off-set was reduced by $5 \mathrm{kWh}$ to $5 \mathrm{kWh}$, where the second, the off-set was removed and the trendline was used directly. The results of these changes to the control equation are also shown in Figure 6-25 and Figure 6-26. In both of these cases, there is a decrease in total energy consumption and greenhouse gas emissions, but an increase in total utility costs and peak energy consumption. Although 
these changes occurred, the overall difference was quite small. In the case of going to no off-set, the change was less than $1 \%$ in each of the parameters, with the exception of peak energy consumption, which increased $\sim 3 \%$. This shows that depending on which variable is of greatest important to minimize, the amount of off-set introduced can be changed to achieve the goals of the system designer.

The overall impact of implementing a predictive control strategy was minimal when using an ice thermal storage system. This was because once the system starts storing the cooling potential as ice, there is no further change in the charge efficiency as the return fluid temperature stays approximately constant. As such, as soon as the predictive control determines a set-point greater than what can be stored sensibly, the increased storage comes with no performance penalty. This differs significantly compared to a system that strictly uses sensible storage. A study by Baldwin and Cruickshank [84] used this same control strategy for a system that used only a sensible storage tank installed in 7 cities across North America and found that the total energy decrease to meet the cooling loads, compared to the standard control strategy, was up to $10 \%$, while total utility bills decreased in every city between $0.3 \%$ and $1.6 \%$. As such, it can be seen that a much larger impact is possible using a predictive control strategy with sensible storage, however a small benefit is still observed when implemented with an ice storage system.

\subsection{Conclusions on the Potential for Off-Setting Peak Loads in Ottawa}

This chapter has provided a step-by-step description as to how the system was developed and how additional components were added onto the system. The impact of each additional component was analyzed to determine its impact on the overall goal of reducing or eliminating the peak loads, while reducing the annual utility costs. The base loads of the 
building were first determined, followed by the potential for offsetting loads using a standard liquid to liquid heat pump with hot and cold thermal storage. Once it was determined that a standard heat pump did not have the temperature range and capability to store the required cooling capacity, a medium temperature chiller was explored, first coupled with sensible storage systems, and then using a latent ice storage system on the cold side.

Through this chapter, the first conclusion that could be made is that off-setting peak cooling loads has the greatest potential for reducing the overall peak demand a house places on the grid. Although the heating load in Ottawa is much more significant compared to the cooling load, $70 \%$ of the cooling load occurs during peak periods, while only $17.5 \%$ of heating loads occur during peak periods. Additionally, the condenser temperature of the heat pump/chiller had a greater impact on the performance compared to the evaporator temperatures. As a result, the increased temperatures, and the small percentage of the heating load during peak periods, the increase in overall increase in total energy consumption to offset the peak consumption is significant. It was found that more than six units of energy must be imputed to the system to offset a single unit of peak energy. Even with time of use billing, it is not foreseeable that cost of electricity would be over six times the price of off-peak costs. This is before looking at the added increase in the system costs, and to be lifetime cost neutral, would need an even larger difference in cost between peak and off-peak electrical rates. Additionally, although peak loads are met with a greater percentage of natural gas fired plants, the only way peak periods would produce six times the greenhouse gas emissions is if the off-peak generation mix remained the same, while peak loads were met using only natural gas (i.e. all nuclear and renewable options were 
shut down during peak periods). This will not occur, and as Ontario continues to increase the percentage of renewables within its grid, and are extending the life of its nuclear generators [85] [86], the shifting of peak heating loads to off-peak periods always increases the greenhouse gas emissions to meet the heating loads. As a result, it was determined that attempting to off-set peak heating loads using a heat pump and thermal storage does not make technoeconomic sense and should not be considered.

The use of cold thermal storage on the other hand has been shown to have significant potential for reducing utility costs and, to a lesser extent, the potential for reducing the greenhouse gas emissions. A comparison between the system using an ice based cold thermal storage with a small pre-heat domestic hot water tank and using just the medium temperature chiller and the base heat pump to directly heat and cool the house is shown in Table 6-8. 
Table 6-8: Summary of results for off-setting peak loads in Ottawa

\begin{tabular}{|c|c|c|c|c|c|}
\hline Performance Metric & $\begin{array}{l}\text { Base - } \\
\text { Heat } \\
\text { Pump }\end{array}$ & $\begin{array}{c}\text { Base - } \\
\text { Medium } \\
\text { Temperature } \\
\text { Chiller }\end{array}$ & $\begin{array}{c}\text { Ice Storage } \\
\text { and DHW } \\
\text { Pre-Heat } \\
\text { Tank }\end{array}$ & $\begin{array}{l}\text { Change } \\
\text { relative to } \\
\text { Heat } \\
\text { Pump }(\%)\end{array}$ & $\begin{array}{l}\text { Change } \\
\text { relative to } \\
\text { Chiller } \\
(\%)\end{array}$ \\
\hline $\begin{array}{l}\text { Peak Consumption } \\
(\mathrm{kWh})\end{array}$ & 4186 & 4686 & 3587 & -14.3 & -26.3 \\
\hline $\begin{array}{l}\text { Off-Peak } \\
\text { Consumption } \\
(\mathrm{kWh})\end{array}$ & 5570 & 6927 & 8238 & 47.9 & 23.5 \\
\hline $\begin{array}{l}\text { Total Consumption } \\
(\mathrm{kWh})\end{array}$ & 9756 & 11,614 & 11,825 & 21.2 & 2.2 \\
\hline $\begin{array}{l}\text { Cooling Peak } \\
\text { Consumption } \\
(\mathrm{kWh})\end{array}$ & 1118 & 1429 & 462 & -58.7 & -86.5 \\
\hline $\begin{array}{l}\text { Cooling Off-Peak } \\
\text { Consumption } \\
(\mathrm{kWh})\end{array}$ & 424 & 546 & 2155 & 408 & 379 \\
\hline Electrical Costs $(\$)$ & 1154 & 1354 & 1292 & 12.0 & -5.4 \\
\hline Cooling Costs $(\$)$ & 219 & 280 & 261 & 19.2 & -8.7 \\
\hline $\begin{array}{l}\text { Greenhouse Gas } \\
\text { Emissions (kg) }\end{array}$ & 574 & 679 & 670 & 16.7 & -1.5 \\
\hline $\begin{array}{l}\text { Cooling Emissions } \\
(\mathrm{kg})\end{array}$ & 104 & 133 & 151 & 45.2 & 17.3 \\
\hline
\end{tabular}

These results showed that the integration of the thermal storage system resulted in a decrease in peak consumption, particularly for cooling. When compared to directly using the medium temperature chiller for heating and cooling, the integration of ice storage resulted in a significant decrease in peak consumption, and a decrease in annual electrical costs and greenhouse gas emissions, and only a small increase in total consumption. Although this showed some promise, the real comparison needs to be made between the regular heat pump, which would typically be used to heat and cool the house and the complete system with the medium temperature chiller and the ice storage system. Based on this comparison, the system as modelled was successfully able to off-set a significant portion of the peak load during cooling periods, however for each unit of peak cooling off- 
set, an increase of 2.64 units of energy are consumed during off-peak periods. A portion of this was also used to preheat the hot water requirements, and when the DHW demand is considered, the offset ratio decreases to 2 units of off-peak energy for every unit of peak energy reduction.

Finally, it is noted that using the medium temperature chiller does not perform as well as the standard heat pump to directly meet the space heating demand of the house. With the assumptions made in terms of the source temperatures and flow to the heat pump and chiller, the change from the base heat pump to the medium temperature chiller resulted in an increase in total space heating consumption of $\sim 1400 \mathrm{kWh}$, or $37 \%$. This increase is responsible for most of the increase in annual utility costs $(\$ 139)$ and greenhouse gas emissions $(76 \mathrm{~kg})$. This increase in heating was a result of a number of individual items when compared to the standard heat pump. These include the selected refrigerant, where r404a, which is designed for low temperature cooling applications, is less effective at providing cooling when compared to the r134a used in the standard heat pump. Additionally, as the unit was designed and optimized for cooling applications, the flooded condenser was not insulated, causing a large heat loss through the shell of the heat exchanger and not through the flowing water, reducing the potential for space heating. Without overcoming this technical obstacle, this system will never be feasible in areas with significant heating loads.

Although this section focused on the economic and greenhouse gas potential for using a heat pump and thermal storage, with limited benefit, a number of additional benefits could be realized by this system. This includes better control of when electricity is used, which depending on future energy policies could see added benefits in future years. 
Additionally, this system could be used to make a building more resilient, as only the fan and pump need to run to provide space heating or cooling. This can easily be achieve with a small generator or battery system, allowing the homeowner to realize the stored heating or cooling during power outages. Finally, this could further reduce a houses reliance on the grid, allowing energy to be stored via renewable sources for later use, and if used in conjunction with a battery system, could allow a home to more easily be off grid compared to storing electricity alone.

In conclusion, it was found that there is potential for offsetting peak cooling loads in Ottawa using a medium temperature chiller connected to a thermal storage system. The required storage and performance could be met using a sensible storage system, however, a tank with very significant volumes (between $1000 \mathrm{~L}$ and $2000 \mathrm{~L}$ ) depending on the setpoint, would be required to provide the necessary storage capacities. This system could be replaced with a $500 \mathrm{~L}$ ice storage system and obtain the same or better results with a much smaller system. This would allow for these systems to be installed as a retrofit, where the access and available space are key factors. Although these systems are technically feasible based on the key assumptions made within the study, it is not economically viable, with the total cost to heat, cool and meet the domestic hot water loads of the building increasing by $12 \%$. Further analysis is needed to determine the impact of the assumptions on the results. Additionally, although the potential for this system to have significant benefit in heating dominated climates may be limited, an analysis of this system across multiple locations in North America should be conducted to determine the its potential, particularly in southern climates. Finally, this analysis only looked at annual operating costs, but the life cycle costs should be examined when determining the potential for this system. 


\section{Chapter 7: Analysis of Different Assumptions, Locations and Rate}

\section{Structures}

In Chapter 6, a detailed description was provided on the development of a medium temperature chiller coupled with thermal storage system(s) to offset the peak electrical loads. The system was examined and developed for use in Ottawa, with a number of assumptions, including the utility structure and availability of source heat. This chapter will build on the findings of Chapter 6 , and examine the impact of the assumptions made, the utility structure, and how the system would function if installed in the same house, but different cities across North America.

\subsection{Impact of Utility Rates}

The first assumption that had to be made was the utility rates used to determine the annual utility costs. As this study started in 2016, and has taken place over multiple rate adjustments, the utility rate at the start of the study was held constant throughout. Between 2016 and 2020, there has been changes in government, each with their own policies and rate adjustments. As such, utility rates dropped for a period between 2017 and 2019 through government subsidies but had recently been brought back to their true costs in late 2019, before a separate rebate is issued to customers. Additionally, only the cost of electricity generation was considered, and not the delivery costs or other associated fees. Depending on the local utility provider, these can be covered as a fixed customer fee(s), a supplemental cost per kWh consumed, or a combination of the two. In the original investigation, none of these fees were considered, but any per kWh fee charged by Hydro Ottawa should also have been included, and the impact of these fees were determined. The original and current utility rates, as well as the delivery fees are shown in Table 7-1. As this consumption would 
occur in Ontario, Canada a 13\% Harmonized Sales Tax (HST) was added to the total rate for 2019 .

Table 7-1: Comparison of utility rates over the course of the study

\begin{tabular}{l|cccc}
\hline $\begin{array}{l}\text { Time of Use } \\
\text { Period }\end{array}$ & $\begin{array}{c}2016 \text { Rates } \\
(\$ / \mathrm{kWh})\end{array}$ & $\begin{array}{c}2019 \text { Rates } \\
(\$ / \mathrm{kWh})\end{array}$ & $\begin{array}{c}\text { Delivery Rates } \\
(\$ / \mathrm{kWh})\end{array}$ & $\begin{array}{c}\text { Total 2019 } \\
\text { Rate }(\$ / \mathrm{kWh})\end{array}$ \\
\hline Peak & 0.18 & 0.208 & 0.0147 & 0.2517 \\
Mid-Peak & 0.132 & 0.144 & 0.0147 & 0.1793 \\
Off-Peak & 0.087 & 0.101 & 0.0147 & 0.1307 \\
\hline
\end{tabular}

Using this updated utility rate for 2019, the baseline utility costs were recalculated when the standard heat pump used, as well as the annual utility costs when utilizing the chiller with the ice storage system. These results are broken down by end use, and the new difference and percent change are calculated in Table 7-2.

Table 7-2: Energy costs using current utility rates

\begin{tabular}{l|cccc}
\hline End Use & Base Case (\$) & $\begin{array}{c}\text { Chiller with } \\
\text { Ice Storage } \\
(\$)\end{array}$ & Change (\$) & $\begin{array}{c}\text { Percent } \\
\text { Change }(\%)\end{array}$ \\
\hline Heating & 551 & 738 & +187 & 34.0 \\
Cooling & 308 & 383 & +75 & 24.5 \\
DHW & 795 & 752 & -43 & -5.4 \\
Total & 1655 & 1874 & +218 & 13.2 \\
\hline
\end{tabular}

With this change in utility rates, the total annual utility costs as calculated for both the base case and the case with the chiller and ice storage increased significantly. The change in utility costs between the two cases remained relatively stable, with the percent difference changing by only $1 \%$ when compared to the 2016 electrical rates. This was 
because although there was an increase in electrical rates, the increase is fairly consistent as a percentage in each time of use category.

\subsubsection{Required Utility Cost Increases}

Based on the current utility rates and source heating assumptions, it was determined to be not economically viable to implement the system, but it is important to determine at what utility rates it would make economic sense in Ottawa. Additionally, many utility providers are encouraging more off-peak consumption, to create a more uniform demand on the electrical grid. As a result, it is foreseeable that in the future, the increase in midpeak and peak utility rates would increase at a rate greater than that of the off-peak rate. If it assumed that the off-peak rate was to remain constant, the impact on the annual utility costs were determined in the case of a $25 \%$ and $50 \%$ increase, as well as a $50 \%$ decrease in off-peak rates and are tabulated in Table 7-3.

Table 7-3: Annual costs based on changing utility rates

\begin{tabular}{l|cccc}
\hline Scenario & $\begin{array}{c}2019 \text { Base } \\
\text { Rate }(\$)\end{array}$ & $\begin{array}{c}25 \% \text { Mid and } \\
\text { Peak Increase } \\
(\$)\end{array}$ & $\begin{array}{c}50 \% \text { Mid and } \\
\text { Peak Increase } \\
(\$)\end{array}$ & $\begin{array}{c}50 \% \text { Off-Peak } \\
\text { Decrease }(\$)\end{array}$ \\
\hline $\begin{array}{l}\text { Base Heat } \\
\text { Pump }\end{array}$ & 1654 & 1886 & 2118 & 1290 \\
$\begin{array}{l}\text { Chiller and } \\
\text { Ice Storage }\end{array}$ & 1873 & 2072 & 2272 & 1335 \\
Difference & 219 & 186 & 154 & 45 \\
\hline
\end{tabular}

This shows that for Ottawa, even with a 50\% increase in only mid-peak and peak rates, or a $50 \%$ decrease in off-peak rates, the system using the chiller and ice storage remains not economically viable. To break even on an annual basis compared to the base 
case, Ontario would need to increase the peak and mid-peak rates by $35 \%$, while simultaneously decreasing the off-peak rate by $50 \%$.

\subsubsection{Impact of Switching to Market Rates}

In Ontario, all residential and small business customers are charged based on a set time of use price, as listed in the preceding section. Large consumers (those using in excess of 250,000 kWh annually) and local distributors are charged based on market prices, which are directly tied to the supply and demand on the utility grid. They have their own definition of peak periods, being 8 AM to 11 PM and off-peak periods of 11 PM to 8 AM and 24 hours a day on weekends, and the average value was calculated monthly by the Independent Electrical Service Operator [87], and are the prices are shown for 2017-2019 in Table 7-4.

Table 7-4: Average market electrical rates by month and rate period in $\$ / M W h$

\begin{tabular}{l|cccccc}
\hline Month & \multicolumn{2}{|c}{2017} & \multicolumn{2}{c}{2018} & \multicolumn{2}{c}{2019} \\
& Peak & Off-Peak & Peak & Off-Peak & Peak & Off-Peak \\
\hline January & 28.56 & 13.6 & 36.98 & 24.35 & 33 & 20.4 \\
February & 26.32 & 15.04 & 22.45 & 14.34 & 32.56 & 22.55 \\
March & 25.45 & 23.6 & 21.48 & 12.39 & 28.74 & 25.01 \\
April & 19.16 & 3.33 & 32.42 & 25.47 & 18.09 & 12.15 \\
May & 6.83 & -1.27 & 16.85 & 6.77 & 9.72 & 4.58 \\
June & 9.14 & 0.5 & 21.49 & 12.57 & 9.12 & -0.68 \\
July & 21.86 & 3.97 & 36.27 & 22.28 & 25.53 & 16.05 \\
August & 24.36 & 7.94 & 35.72 & 22.81 & 20.76 & 9.91 \\
September & 29.13 & 13.33 & 32.03 & 23.21 & 18.88 & 8.97 \\
October & 10.6 & 5.77 & 16.35 & 9.57 & 10.91 & 2.63 \\
November & 19.07 & 7.18 & 34.07 & 13.62 & 26.48 & 13.62 \\
December & 25.94 & 14.76 & 34.85 & 20.91 & 25.55 & 16.84 \\
\hline
\end{tabular}

Using these values, the overall electrical cost for heating, cooling and the domestic hot water loads of the building were calculated using market rates for each of the last three 
years. Additionally, the annual utility rates using the chiller and ice storage and market rates was also calculated and these values are in Table 7-5.

Table 7-5: Annual Utility costs using the Ontario market rate

\begin{tabular}{l|ccc}
\hline Scenario & $\begin{array}{c}\text { 2017 Market } \\
\text { Prices }(\$)\end{array}$ & $\begin{array}{c}\text { 2018 Market } \\
\text { Prices }(\$)\end{array}$ & $\begin{array}{c}\text { 2019 Market } \\
\text { Prices }(\$)\end{array}$ \\
\hline $\begin{array}{l}\text { Base Heat } \\
\text { Pump }\end{array}$ & 1493 & 2227 & 1735 \\
$\begin{array}{l}\text { Chiller and } \\
\text { Ice Storage }\end{array}$ & 1641 & 2541 & 1996 \\
Difference & $148(10 \%)$ & $314(14 \%)$ & $261(15 \%)$ \\
\hline
\end{tabular}

The first result observed is that when using market rates, significant fluctuations in annual pricing can occur, as the individual pricing is completely dependent on the demand during a given year, which is predominantly driven by the weather conditions in the province. This can be seen in 2018 in particular, which had a historically hot summer [88], and as such, prices remained extremely high through the cooling season when compared to the pricing in 2017 and 2019. This is one of the main drivers in fixed time of use pricing, allowing cost certainty to consumers that market pricing cannot provide. All that said, in all three years in which the market rate pricing was explored, the annual utility costs increased when shifting from the base heat pump to the chiller with ice storage compared to using fixed time of use rates. When looking at the underlying values, it appears much of this increase is as a result of the increased electricity required to meet the space heating load, and to prove this point, the cost to meet just the DHW and space cooling loads were calculated and are shown in Table 7-6. 
Table 7-6: Annual costs to meet the space cooling and DHW load

\begin{tabular}{l|ccc}
\hline Scenario & $\begin{array}{c}\text { 2017 Market } \\
\text { Prices (\$) }\end{array}$ & $\begin{array}{c}\text { 2018 Market } \\
\text { Prices (\$) }\end{array}$ & $\begin{array}{c}\text { 2019 Market } \\
\text { Prices (\$) }\end{array}$ \\
\hline $\begin{array}{l}\text { Base Heat } \\
\text { Pump }\end{array}$ & 952 & 1465 & 1046 \\
$\begin{array}{l}\text { Chiller and } \\
\text { Ice Storage }\end{array}$ & 911 & 1538 & 1070 \\
Difference & $-41(-4 \%)$ & $73(5 \%)$ & $24(2 \%)$ \\
\hline
\end{tabular}

These results show that when using market rates, the cooling and DHW costs can decrease when going from the base case to the chiller and ice storage, and even in years when the cost increased, it was a smaller increase then what was seen with the standard time of use rates. From this, it can be concluded that the real driver in cost increase is the much lower heating performance of the chiller when compared to the base heat pump. As a result, no matter what cost scheme is used, without overcoming the heating performance of the chiller, this system will not be economically viable in heating dominated climates. Additionally, these results did show that if market pricing is introduced to residential customers, and not just large consumers there is potential for decreasing the annual costs associated with cooling and DHW. Although this may not be feasible in heating dominated climates, this shows that there is significant potential in cooling dominated climates.

A significant challenge of determining the impact of the use of market prices, while modelling system performance is that the market rate is predominantly driven by weather conditions in Ontario. As such very hot or cold temperatures across the province cause a spike in demand and consequently a spike in market rates. The challenge is the annual performance is dictated by fixed, CWEC data, and as such, the peak demands may not coincide with the peak market rates which would occur during these extreme weather conditions. To truly determine the impact of market rates, the models must be run with 
actual weather data that coincides with the market electrical rates. Some historic temperature data is publicly available, but a large portion of the building loads are driven by solar gains, and as such, without accurate temperature and solar data, it is impossible to definitively determine the potential for cost savings through demand side management using market rates. The use of average monthly rates reduced this error slightly, and provides a good estimate, but to determine the true impact, models need to be run with matched weather data and market rates.

\subsection{Impact of Source Assumptions on Heating Performance}

Through the economic analysis completed in Chapter 6 and the preceding section (7.1), it has been shown that there is potential for reducing the costs of space cooling and DHW through the use of the chiller and ice storage, but has yet to be realized under modelled conditions. However, when the total utility costs are examined, the space heating costs dominate in Ottawa due to its heating dominated climate. The decrease in heating performance of the chiller when compared to the base heat pump is the main reason the system is not economically feasible. When looking at what impacts the heating performance of the system, the single variable with the greatest impact is the evaporator temperature, which is dictated by the available heat source. As the heat source fell outside the scope of this study (many theses have been completed solely on the modelling of

sources of energy for heat pumps), an assumption was made for the source temperature and flow. For all the annual models run in this study, for both the base scenarios and the 
scenarios that incorporate thermal storage, a constant source temperature of $15^{\circ} \mathrm{C}$ was selected and utilized.

This source temperature of $15^{\circ} \mathrm{C}$ is a high source temperature, particularly since it is supplied continuously year-round. To meet the space heating loads this meant that a lift through the heat pump of only $8-10^{\circ} \mathrm{C}$ was required, which is small and easily achieved in an efficient manner. The standard heat pump used refrigerant $134 \mathrm{a}$ which is designed for use with small temperature lifts and within those higher evaporator temperatures. The chiller uses refrigerant 404a, which is designed for use with lower evaporator temperatures, but comparatively has lower performance at higher evaporator temperatures when compared to $134 \mathrm{a}$. As such, selecting a source temperature of $15^{\circ} \mathrm{C}$ favored the base heat pump and created a significant increase in the required electricity to meet the space heating demands. As such, more investigation is required to determine the impact this source temperature on the overall results, and whether it was a fair assumption.

Heat pumps can run with a wide range of heat sources, including outdoor air, ground source, solar or district heating loops. Air source was not explored as the system required a liquid to liquid heat pump and the ability to integrated liquid based thermal storage systems. Additionally, air source heat pumps have the lowest source temperatures, and can only achieve temperatures equal to the outdoor temperature. Ground source and solar systems have a wide range of source temperatures and vary throughout the year depending on the amount of energy used and weather conditions. Ground source heat pumps in Canada typically see return temperatures in the winter as low as $-2^{\circ} \mathrm{C}$ to $4{ }^{\circ} \mathrm{C}$, depending on location, piping layout, depth and ground water present [89]. The temperature peaks during the summer months, and then decrease through the winter months 
as heat is extracted from the ground and exterior temperatures decrease. District heat on the other hand supplies the highest source temperatures and that depend on their source of heat (industrial waste heat, a boiler, solar etc.). Based on these parameters, the selected $15^{\circ} \mathrm{C}$ source temperature could be accurate if connected to a district heating system but is higher than would have been expected if connected to a ground loop. As such, a sensitivity study was conducted to first determine the impact of the source temperature on the base energy consumption using a constant source temperature of $5^{\circ} \mathrm{C}$ and $10^{\circ} \mathrm{C}$. The results, broken down by peak, mid-peak and off-peak consumption in Figure 7-1, and by end use in Figure 7-2.

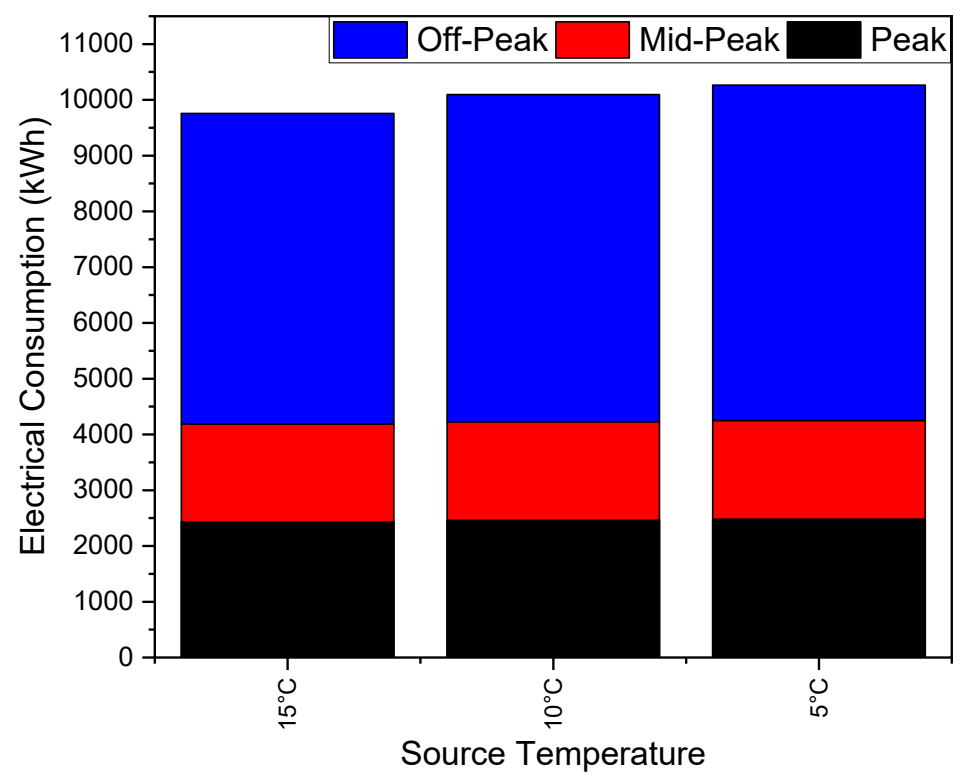

Figure 7-1: Electrical consumption by time of use for different source temperatures 


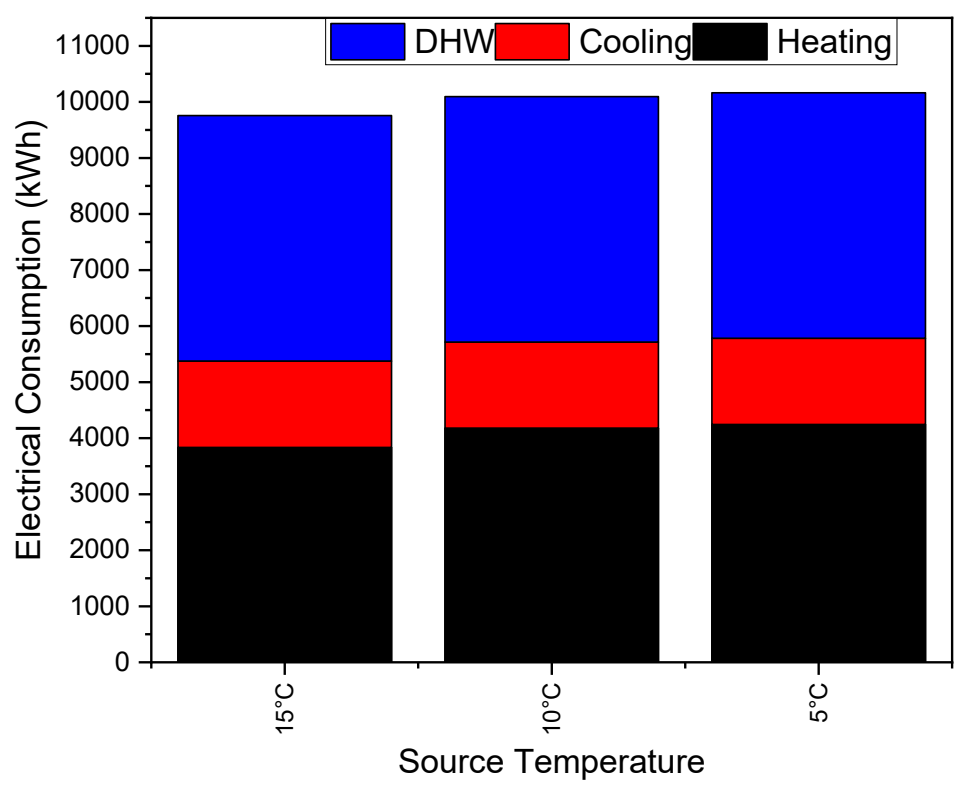

Figure 7-2: Electrical consumption by end use for different source temperatures

These results show that the decrease in source temperature did result in an increase in the overall energy consumption as a result of an increase in the required input to meet the space heating demand. This increase equated to $5.2 \%$ of total consumption from a source temperature of $15^{\circ} \mathrm{C}$ to $5^{\circ} \mathrm{C}$. This decrease would be even more significant if the source temperature were to further decrease, however during experimental testing of the heat pump, the heat pump shut down due to low pressure at source temperatures that occurred when using the glycol solution, indicating it has not been designed to operate at temperatures below $5^{\circ} \mathrm{C}$. After the impact of the source temperature was determined on the base case, the same source temperatures, as well as $0^{\circ} \mathrm{C}$, were plotted as shown in Figure 7-3. 


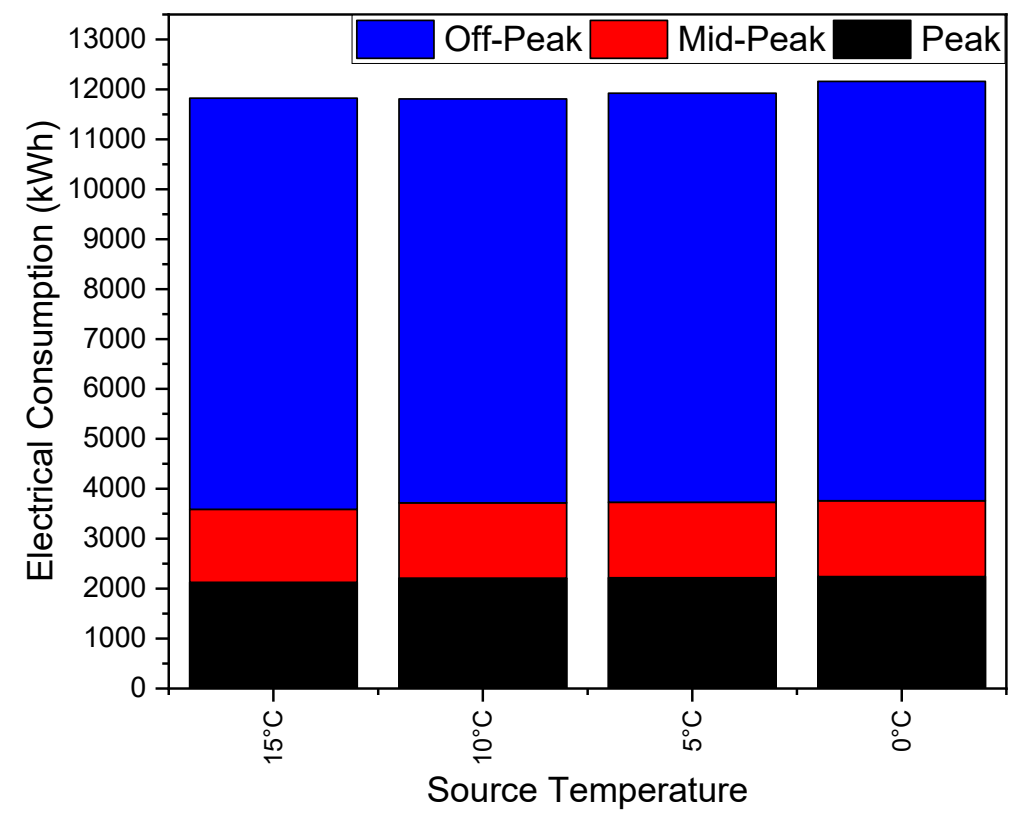

Figure 7-3: Electrical consumption by time of use for the medium temperature chiller with different source temperatures

Based on these results, it can be seen that, like the base case, the total energy consumption increased as the source temperature decreased, but only by $0.8 \%$ from $15^{\circ} \mathrm{C}$ to $5^{\circ} \mathrm{C}$ source temperature, and a further $2 \%$ when $0^{\circ} \mathrm{C}$ source temperature is utilized. To compare the results at each of the three source temperatures, Table 7-7 was created to show the difference between the base case and the system utilizing the chiller and ice storage system. 
Table 7-7: Comparison between base case and system using ice storage and the medium temperature chiller at different source temperatures

\begin{tabular}{l|cccc}
\hline Scenario & $\begin{array}{c}\text { Base } \\
\text { Consumption } \\
(\mathrm{kWh})\end{array}$ & $\begin{array}{c}\text { Complete } \\
\text { System }(\mathrm{kWh})\end{array}$ & $\begin{array}{c}\text { Increase } \\
(\mathrm{kWh})\end{array}$ & $\begin{array}{c}\text { Percent } \\
\text { Increase }(\%)\end{array}$ \\
\hline $\begin{array}{l}15^{\circ} \mathrm{C} \text { Source } \\
(\mathrm{kWh})\end{array}$ & 9756 & 11825 & 2069 & $21 \%$ \\
$\begin{array}{l}10^{\circ} \mathrm{C} \text { Source } \\
(\mathrm{kWh})\end{array}$ & 10096 & 11807 & 1711 & $17 \%$ \\
$\begin{array}{l}5^{\circ} \mathrm{C} \mathrm{Source} \\
(\mathrm{kWh})\end{array}$ & 10263 & 11923 & 1660 & $16 \%$ \\
\hline
\end{tabular}

These results show that the source side temperature does impact the consumption in both the base case and the tested scenario, and the increase is much larger in the standard heat pump compared to the medium temperature chiller. When the complete system integrating the ice thermal storage system is tested at lower source temperatures, the relative increase in comparison to the base model has decreased by $\sim 400 \mathrm{kWh}$, and the difference between the two systems has decreased to $16 \%$. This is caused by both the lower performance of the chiller at higher source temperatures when compared to the standard liquid to liquid heat pump, and the quicker decrease in performance of the standard heat pump as the source temperature decreases. Even with this relative change in performance between the two systems with the change in source temperatures, the change is not significant enough to change the overall conclusions that have been made in terms of the potential for this system to be economically feasible.

In addition to the source side temperature, a second assumption had to be made in terms of how heat is dissipated from the condenser during the cooling season. In both the base case and the system using the heat pump and thermal storage, an outdoor air to liquid heat dissipation was assumed. This provided a return temperature to the heat pump slightly above the outdoor dry bulb temperature and is a common method for dissipating heat in 
residential applications and has the highest return temperatures of any heat dissipation method. The alternative heat dissipation that could be employed would be through a ground loop, which would be used if a ground loop had been installed. In that case, the dissipated heat would be rejected back to the ground, helping to increase the ground temperature and allowing the boreholes to store energy for use during the heating season. If that were to be the case, lower, consistent temperatures would be available to reject the excess heat, and the energy for space cooling would decrease. Like the decrease in source temperature, this would introduce an off set into the data, but the relative performance between the two heat rejections temperatures would remain constant.

Based on this section, it has been shown that the assumption made in terms of the source and heat rejection temperatures have an impact on the required energy to meet the building energy demands. Although this does impact overall consumption, it was found that this assumption did not impact the analysis of the potential for demand side management using a medium temperature chiller and thermal storage systems. This is a result of the fact the source and heat rejection temperatures impacted the electrical consumption, but the relative change is not significant enough to significantly impact the results obtained for Ottawa. As such, the conclusions made hold true, no matter what type or temperature of source heat is supplied to the system. The magnitude of the obtained results may change depending on the assumptions made, but the fact the system is unable to economically off-set peak electrical loads holds true no matter what heating and heat rejections systems it is connected to. 


\subsection{Potential for Off-Setting Peak Loads across North America}

To this point, the analysis of the potential to achieve demand side management using the chiller and thermal storage, including the ice storage system has focused on a residence located in Ottawa, Canada. Through these results, it was found that there is significant potential for offsetting the peak cooling loads through this system, but the degradation in heating performance removed any potential for the overall system. These results were for Ottawa, but potentially other locations within North America, which have both different utility rates and rate structures, as well as mix of heating and cooling loads would have a different outcome. As such, it was decided that locations across North America would be tested, and to ensure climatic diversity, a city was selected from for each ASHRAE [90] climate zone, from zone 1 to zone 7 (no city was selected for zone 8 as no cooling is typically present). Within these climate zones, the lower the number, the higher the cooling loads and lower the heating loads to zone 8 where there is almost no cooling load and the building loads are heating dominated. As such, lower climate zones are located in the southern areas, increasing in number further north.

In addition to deciding that a climate zone would be tested, individual cities or geographical regions within each zone had to be selected. To be considered, the local utility had to either bill by, or provide customers the option to be billed by a time of use billing strategy. Taking into account these two parameters, the most populous cities meeting these criteria in each climate zone were examined and one was selected for each zone for evaluation. The selected cities (climate zone) were Miami (1), Phoenix (2), Los Angeles 
(3), Portland (4), Boston (5), Toronto (6) and Sudbury (7). These cities, as well as the ASHRAE climate zones are shown in Figure 7-4.

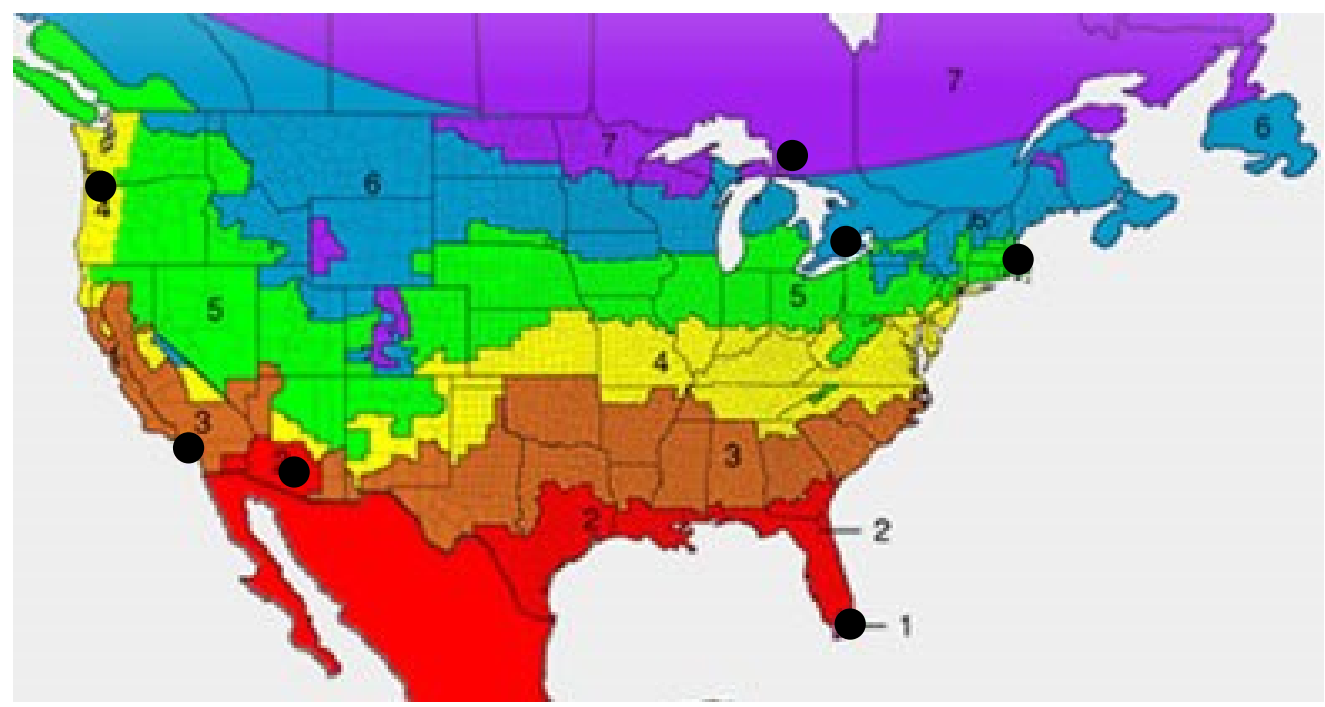

Figure 7-4: Selected cities and the ASHRAE climate map for North America

Once the cities were selected, the electrical rates and peak periods for each provider had to be determined. To keep consistent through the study, rates were obtained in March 2017, and were held consistent through the remainder of this thesis. Additionally, as locations in both the United States and Canada were observed, local currencies were utilized with no conversions to eliminate possible changes over time as economies changes cause a change in conversation rates. The rates for the selected cities are shown in Table 7-8, while the rate periods are shown in Table 7-9 through Table 7-14. In these tables, all times are in the 24 hour clock, and are valid only for weekdays, where all weekends are off peak rates, with the exception of Portland, where mid peak rates are valid on Saturday from $6 \mathrm{hr}-22 \mathrm{hr}$. 
Table 7-8: Utility rates for selected cities $[91,92,93,94,95,96,97]$

\begin{tabular}{lcccc}
\hline Location & $\begin{array}{c}\text { ASHRAE } \\
\text { Climate } \\
\text { Zone }\end{array}$ & $\begin{array}{c}\text { Peak Rate } \\
(\$ / \mathrm{kWh})\end{array}$ & $\begin{array}{c}\text { Mid-Peak Rate } \\
(\$ / \mathrm{kWh})\end{array}$ & $\begin{array}{c}\text { Off-Peak Rate } \\
(\$ / \mathrm{kWh})\end{array}$ \\
\hline Miami, Florida & 1 & 0.19165 & $\mathrm{~N} / \mathrm{A}$ & 0.0331 \\
Phoenix, Arizona & 2 & $0.1020-0.2226$ & $\mathrm{~N} / \mathrm{A}$ & $0.0711-0.0741$ \\
Los Angeles, California & 3 & $0.1456-0.2433$ & $0.1456-.01641$ & $0.1396-0.1492$ \\
Portland, Oregon & 4 & 0.1998 & 0.1428 & 0.0421 \\
Boston, Massachusetts & 5 & 0.1973 & $\mathrm{~N} / \mathrm{A}$ & 0.0920 \\
Toronto, Ontario & 6 & 0.1564 & 0.1194 & 0.0894 \\
Sudbury, Ontario & 7 & 0.1504 & 0.1134 & 0.0834 \\
\hline
\end{tabular}

Table 7-9: Rate periods for Miami

\begin{tabular}{l|ll}
\hline Rate Period & Summer (Apr 1- Oct 31) & Winter (Nov 1-Mar 31) \\
\hline Peak & $12-21$ & $6-10$ and 18-22 \\
Off Peak & $0-12$ and 21-24 & $0-6,10-18$ and 22-24 \\
\hline
\end{tabular}

Table 7-10: Rate period for Phoenix

\begin{tabular}{l|ccc}
\hline Rate Period & $\begin{array}{c}\text { Summer } \\
\text { (Jul 1 - Aug 31) }\end{array}$ & $\begin{array}{c}\text { Shoulder } \\
\text { (May 1 - Jun 30 and } \\
\text { Sep 1 - Oct 31) }\end{array}$ & $\begin{array}{c}\text { Winter } \\
\text { (Nov 1 - Apr 30) }\end{array}$ \\
\hline Peak & $13-20$ & $13-20$ & $5-9$ and 17-21 \\
Off Peak & $0-13$ and 20-24 & $0-13$ and 20-24 & $0-5,9-17$ and 21-24 \\
\hline
\end{tabular}

Table 7-11: Rate periods for Los Angeles

\begin{tabular}{l|c}
\hline Rate Period & All Year \\
\hline Peak & $13-17$ \\
Mid Peak & $10-13$ and $17-20$ \\
Off Peak & $0-10$ and $20-24$ \\
\hline
\end{tabular}

Table 7-12: Rate periods for Portland

\begin{tabular}{l|ll}
\hline Rate Period & Summer (May 1- Oct 31) & Winter (Nov 1-Apr 30) \\
\hline Peak & $15-20$ & $6-10$ and 17-20 \\
Mid Peak & 6-15 and 20-22 & $10-17$ and 20-22 \\
Off Peak & $0-6$ and 22-24 & $0-6$ and 22-24 \\
\hline
\end{tabular}


Table 7-13: Rate periods for Boston

\begin{tabular}{l|c}
\hline Rate Period & All Year \\
\hline Peak & $8-21$ \\
Off Peak & $0-8$ and 21-24 \\
\hline
\end{tabular}

Table 7-14: Rate periods for Toronto and Sudbury

\begin{tabular}{l|ll}
\hline Rate Period & Summer (May 1- Oct 31) & Winter (Nov 1 - Apr 30) \\
\hline Peak & $11-17$ & $7-11$ and 17-19 \\
Mid Peak & $7-11$ and 17-19 & $11-17$ \\
Off Peak & $0-7$ and 19-24 & $0-7$ and 19-24 \\
\hline
\end{tabular}

After the energy structure was determined for each location, the base energy consumption was determined. The same house model that was used for the analysis in Ottawa was utilized for each of the seven locations, with the weather files changed for each location. For each of the Canadian locations, CWEC files for the cities were used, while for American cities, Typical Meteorological Year (TMY2) files were used [98]. The standard liquid to liquid heat pump was used to determine the base heating and cooling loads, with source heating temperature of $15^{\circ} \mathrm{C}$ and using the outdoor dry bulb temperature as the heat sink temperature. The results by building load are shown in Figure 7-5, while the breakdown by time of use and the cost, is shown in Figure 7-6. 


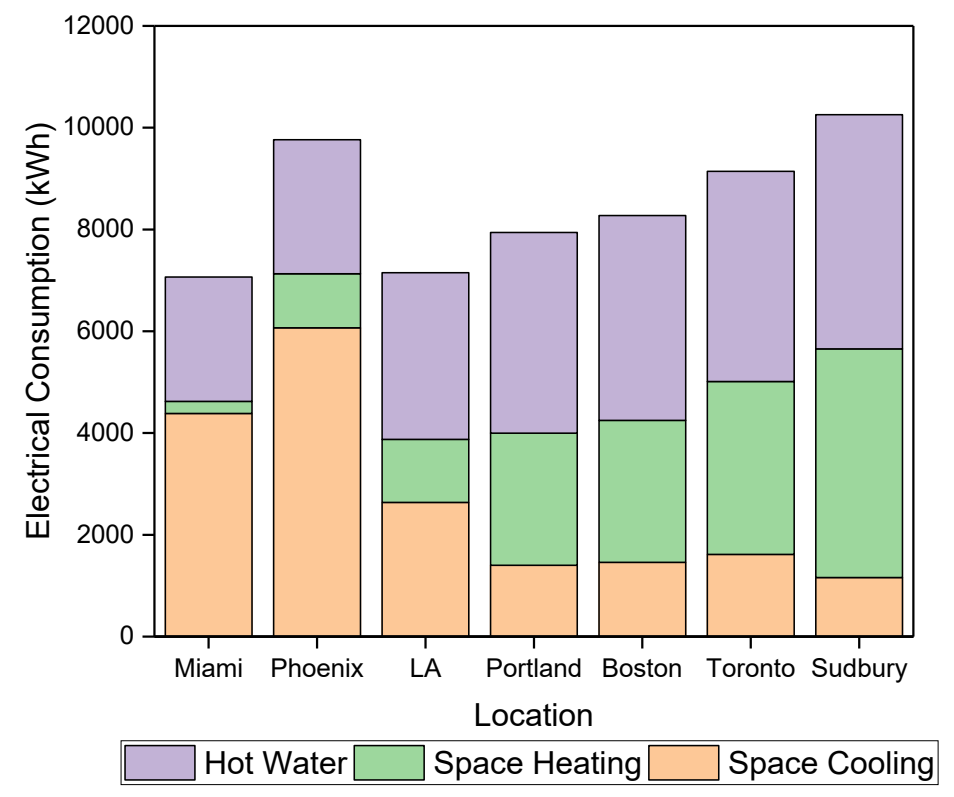

Figure 7-5: Annual electrical consumption by end use

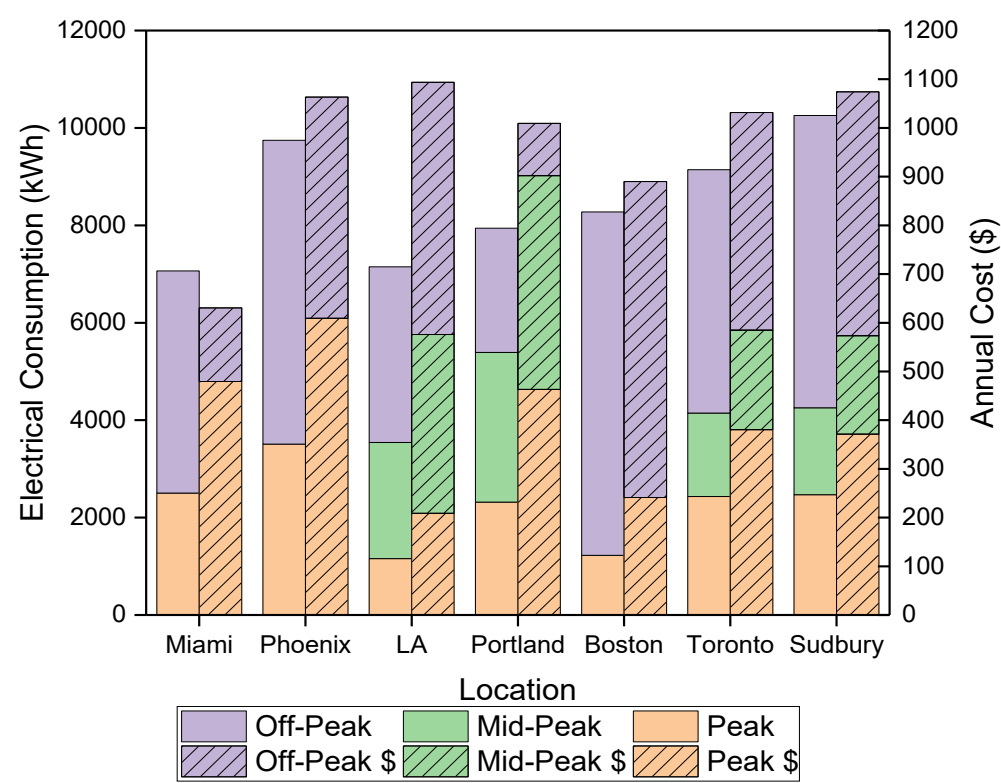

Figure 7-6: Annual consumption and cost by time of use

From these results, it can be seen that as the ASHRAE climate zone number increases (going left to right on each graph), the proportion of heating to cooling of the building increases. It can also be noted that in all locations, the peak and mid-peak costs were disproportionately higher than the amount of energy used during the same period. 
Additionally, in general, the higher the percentage of the building load that is attributed to space cooling, the greater the percentage of utility costs as a result of energy used during peak periods.

\subsubsection{Offsetting Cooling with Sensible Storage}

Before going directly to an ice based thermal storage system, the potential for offsetting the peak cooling loads in each location with a sensible storage tank using a glycol/water solution was examined. For each location, a parametric study was conducted, varying the volume and the set-point of the storage tank. For the tank set-point, two setpoints were utilized, one during peak periods, which was held constant at $15^{\circ} \mathrm{C}$ for all scenarios, and an off-peak set-point which was varied from $-10^{\circ} \mathrm{C}$ to $5^{\circ} \mathrm{C}$ in $5^{\circ} \mathrm{C}$ increments. The tank volume was simultaneously varied in $0.25 \mathrm{~m}^{3}$ increments from $0.25 \mathrm{~m}^{3}$ to $3 \mathrm{~m}^{3}$, creating 48 simulations for each location. The simulated energy results were then converted to an annual utility costs and the calculated annual utility costs were graphed using a surface plot with set-point and tank volume as the $\mathrm{X}$ and $\mathrm{Y}$ variables. Two example graphs for Phoenix and Portland are shown in Figure 7-7 and Figure 7-8. 


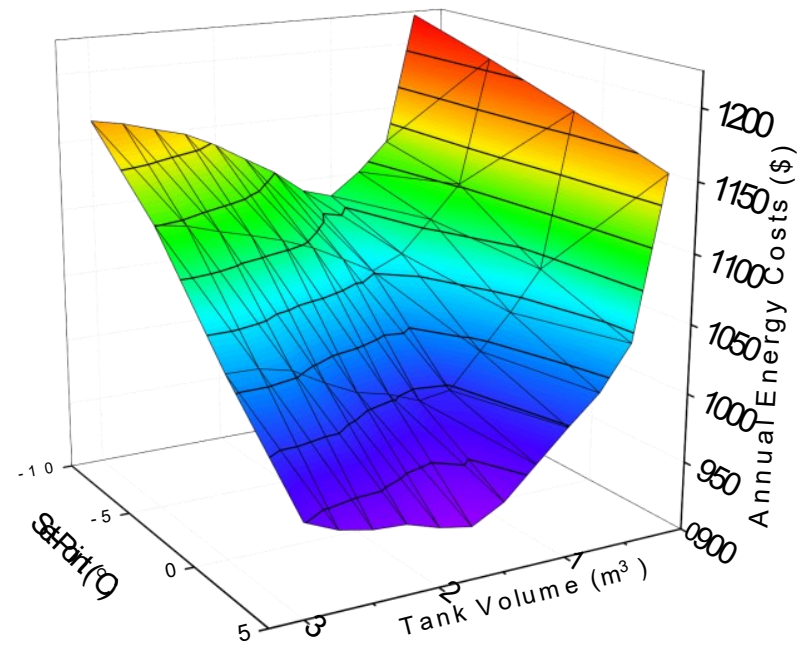

Figure 7-7: Impact of changing set-point and tank volume and annual utility rates for Phoenix

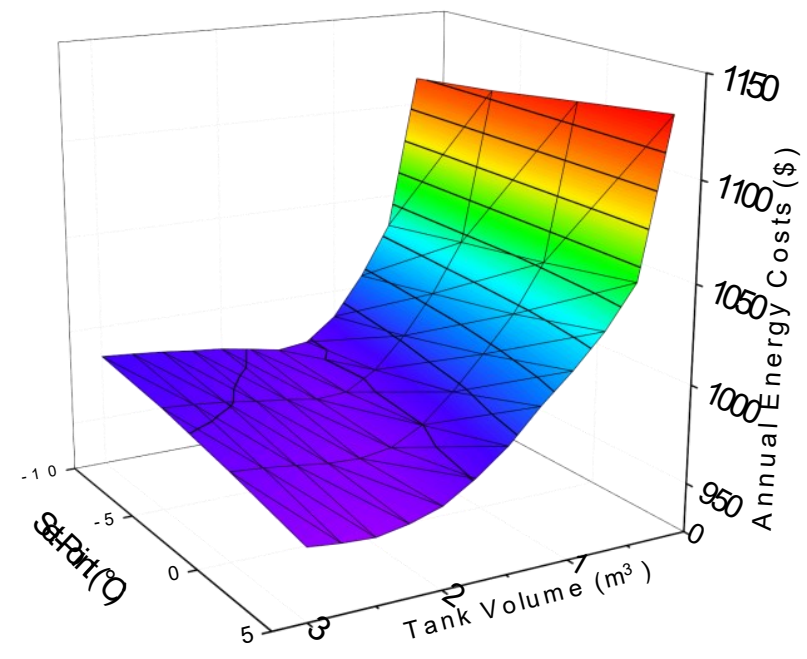

Figure 7-8: Impact of changing set-point and tank volume and annual utility rates for Portland

From this series of graphs, it can be seen that the optimal configuration for any given location is dependent on both the cooling loads of the building, the ratio between peak and off-peak utility costs, and the total energy consumption. In all cases, it was concluded that it is paramount to find the correct storage capacity, as if not enough storage is integrated into the system, the amount of peak energy that is off-set is minimal, 
increasing the annual energy costs. On the other hand, it is seen that a second spike in energy costs occurs in most locations if too much storage is integrated into the system, as the chiller must constantly operate at lower temperatures, near the set-point of the storage tank, without realizing the stored cooling potential. This decrease in operating temperature resulted in a significant drop in chiller performance, and consequently an increase in electrical energy required. Additionally, a trend towards higher set-point temperatures seeing lower annual costs was observed, as again, the performance of the chiller decreases with the lower operating temperatures. In contrast however, the higher the set-point temperature, the larger the tank volume typically required, and as such, more space and a higher initial capital cost is required.

Taking the results from each location, the optimal tank size and tank set-point was determined for each of the seven locations. When results from multiple configurations provided the same, or very similar results, the configuration in which the tank size was smallest was selected to reduce the required volume within the system. The optimal configuration in which annual energy costs are minimized for each location, and a comparison of cost compared to baseline for each of the locations is shown in Table 7-15.

Table 7-15: Optimal parameters and the comparison between base costs and using demand side management

\begin{tabular}{lccccc}
\hline Location & $\begin{array}{c}\text { Optimal Tank } \\
\text { Volume }\left(\mathrm{m}^{3}\right)\end{array}$ & $\begin{array}{c}\text { Optimal Set- } \\
\text { Point }\left({ }^{\circ} \mathrm{C}\right)\end{array}$ & $\begin{array}{c}\text { Annual Energy } \\
\text { Cost }(\$)\end{array}$ & $\begin{array}{c}\text { Baseline Energy } \\
\text { Cost }(\$)\end{array}$ & $\begin{array}{c}\text { Difference } \\
(\%)\end{array}$ \\
\hline Miami & 1.50 & 0 & 446.39 & 630.44 & $-29.19 \%$ \\
Phoenix & 1.75 & 5 & 937.65 & 1063.40 & $-11.83 \%$ \\
Los Angeles & 0.50 & 5 & 1083.40 & 1093.78 & $-0.95 \%$ \\
Portland & 2.00 & 0 & 963.93 & 1009.48 & $-4.51 \%$ \\
Boston & 1.50 & 5 & 954.81 & 890.11 & $7.27 \%$ \\
Toronto & 1.25 & 0 & 1104.57 & 1031.52 & $7.08 \%$ \\
Sudbury & 1.00 & 5 & 1175.67 & 1074.24 & $9.44 \%$ \\
\hline
\end{tabular}

These optimal results for tank volume and set-point, and consequently the storage 
capacity were dependent on the total peak cooling load, the ratio between peak and off peak rates, and the daily duration of peak periods. Further to these optimal results for each location and the annual utility costs, the annual optimal consumption and utility costs, based on the time of use, and their comparison to the base case for both are shown in Figure 7-9 and Figure 7-10 respectively.

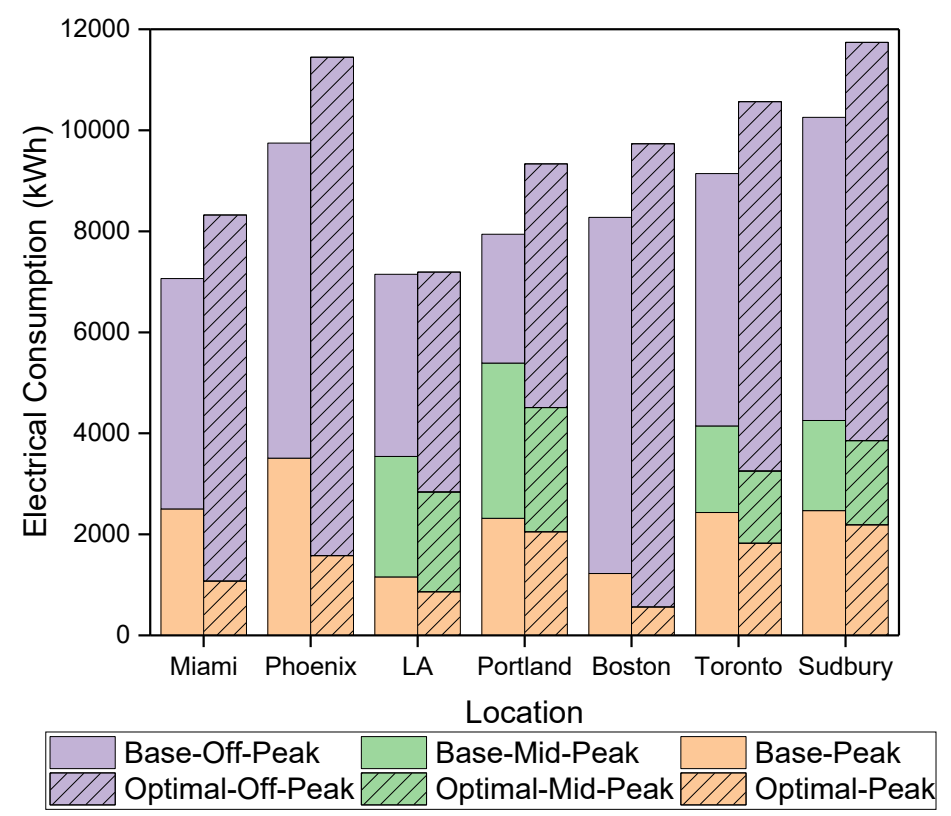

Figure 7-9: Comparison of electrical consumption by time of use 


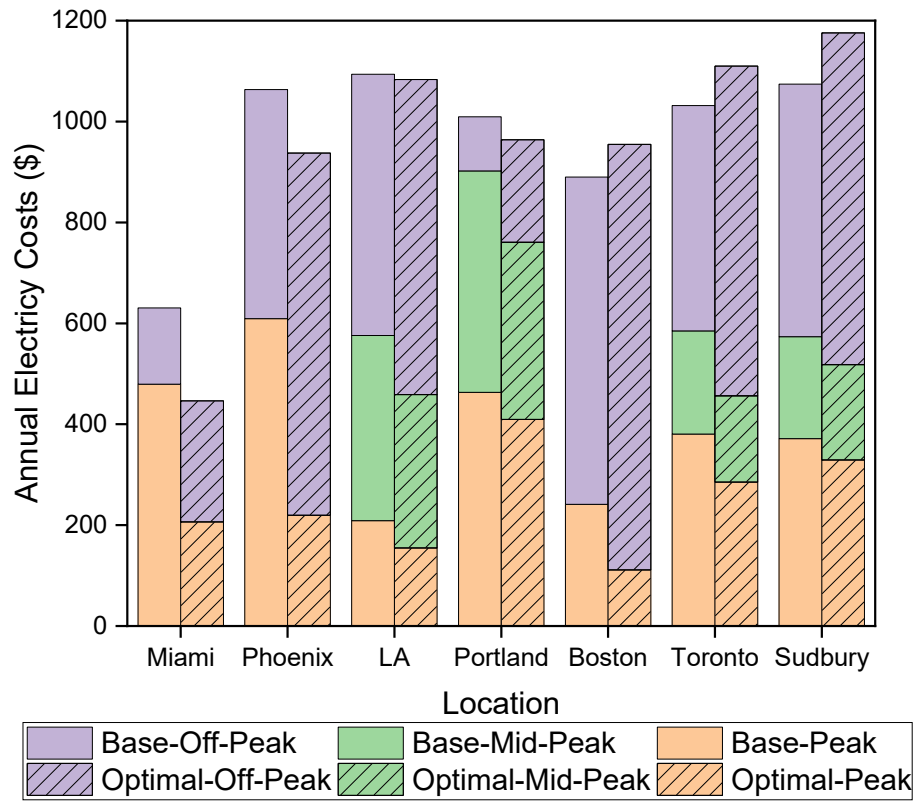

Figure 7-10: Comparison of annual electrical cost by time of use

Based on these results, a number of trends were observed. The first is the potential is greatest for offsetting significant peak loads in regions where building loads are dominated by cooling. In northern climates a substantial reduction in peak loads can be obtained, however the economic benefits are not there when compared to southern climates. There are two factors influencing this. The first is the larger percentage of the building load that can be off-set and shifted to off-peak periods, and the second being a smaller increase in the heating loads as a result of using the chiller.

Even though climate appeared to be the most significant factor in determining the feasibility, the ratio between utility rates between peak and off-peak periods influenced the optimal configuration of the system. This can be seen in particular in the results for Los Angeles, where the small difference in peak to off-peak rates creates little economic benefits even though a high cooling load exists. When the ratio is high (peak to off-peak), it was beneficial to increase the storage capacity to offset as much of the peak load as possible, even if the increase in off-peak consumption is disproportionately large. In all 
locations, although the peak load is reduced, an increase in the overall annual energy consumption is also observed as a result of the decreased performance of the chiller in charging the thermal storage.

In each case, with the exception of Los Angeles, to achieve the optimal economic benefits, significant volumes of at least $1000 \mathrm{~L}$ is required. When lower volumes with colder storage set-points are used, the degradation in performance based on the lower evaporator temperatures decreases the economic performance of the system. As discussed when determining the performance of the system in Ottawa, there is limited possibility to incorporate the volumes required for sensible thermal storage in residential applications and as such, the use of ice storage is required in these locations as well.

\subsubsection{Use of Ice Storage in Multiple Locations Across North America}

Once it was determined that there is a potential economic benefit to this system across multiple locations in North America, but with unachievable volumes within residential applications, the potential for using ice storage was explored. The final ice storage system developed for use in Ottawa was integrated into the model for each location. Additionally, the integration of the domestic hot water pre-heat tank was also incorporated into the new model. Finally, for an added increase in economic benefits, the use of forecasted predicted high temperatures to determine the amount of cooling was also incorporated into each location. This was undertaken for all locations studied with the exception of Los Angeles, where it was found that the optimal storage volume is only $500 \mathrm{~L}$. This was caused by a very small ratio between the peak and off-peak electrical rates, and there is minimal benefit to shifting electricity with this rate structure. Additionally, the 
ice storage has a similar volume to the optimal tank volume, and as such no additional benefit could be realized.

The first step was to determine the relationship for each location between the daily maximum temperature and the peak cooling load for that day. The same method was used as that for developing the relationship for Ottawa, where the peak cooling load was plotted vs. the daily maximum temperature and a linear trendline was derived for each location. Figure 7-11 shows an example graph for Portland.

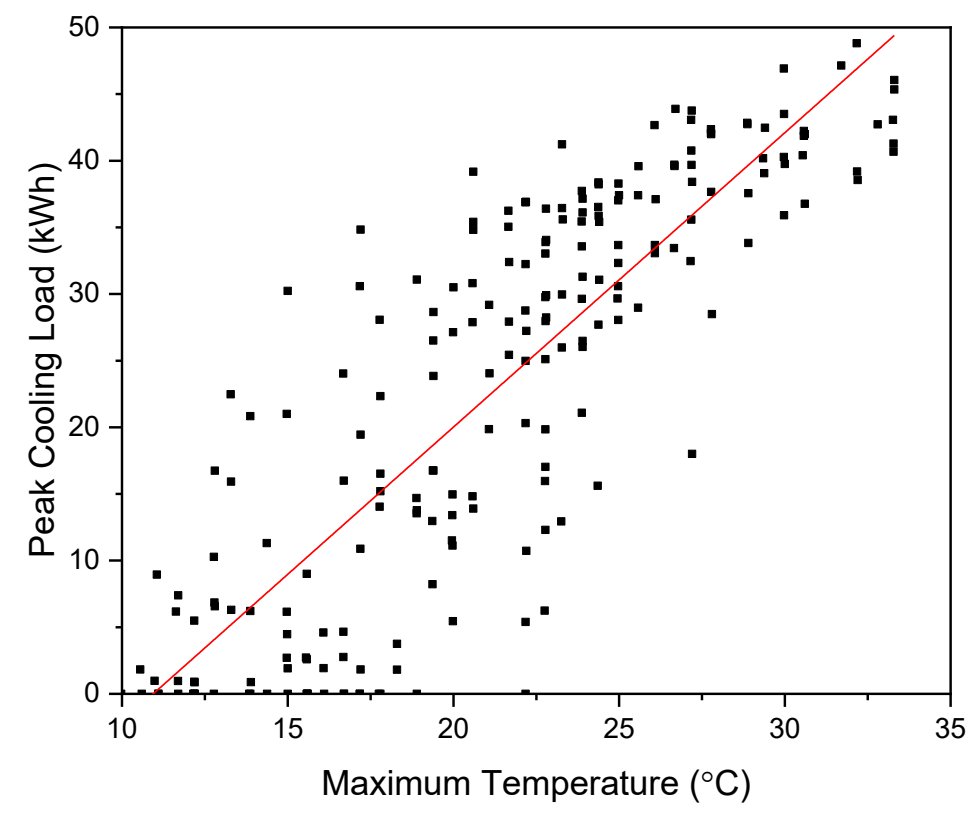

Figure 7-11: Relationship between peak cooling load and maximum daily temperature for Portland

Similar graphs and relationships were developed for each of the seven locations being studied, and those relationships were compiled in Table 7-16. 
Table 7-16: Equations for each of the cities relating max daily temperature in degrees Celsius and daily peak cooling load in $\mathrm{kWh}$

\begin{tabular}{l|c}
\hline \multicolumn{1}{c|}{ Location } & $\begin{array}{c}\text { Relationship between Daily Max and } \\
\text { Cooling Load }\end{array}$ \\
\hline \multirow{2}{*}{ Miami, FL } & $L_{\mathrm{C}}=1.56 T_{\max }-21.1(\mathrm{~S})$ \\
& $L_{\mathrm{C}}=0.62 T_{\max }-12.3(\mathrm{~W})$ \\
\hline \multirow{2}{*}{ Phoenix, AZ } & $\begin{array}{c}L_{\mathrm{C}}=0.37 T_{\max }+10.88(\mathrm{~S}) \\
\end{array}$ \\
\hline Los Angeles, CA & $L_{\mathrm{C}}=0.026 T_{\max }{ }^{2}-0.55 T_{\max }+3.05(\mathrm{~W})$ \\
\hline Portland, OR & $L_{\mathrm{C}}=0.91 T_{\max }-1.42$ \\
\hline Boston, MA & $L_{\mathrm{C}}=2.21 T_{\max }-24.17$ \\
\hline Toronto, ON & $L_{\mathrm{C}}=1.26 T_{\max }-4.55$ \\
\hline Sudbury, ON & $L_{\mathrm{C}}=1.21 T_{\max }-3.94$ \\
\hline
\end{tabular}

In the case of Miami and Phoenix, cooling season lasts all year, however there are two different definitions of peak periods for the winter and cooling season, and as such, the amount cooling which occurs during peak period changes depending on the season, and are denoted as summer (S) and winter (W). The simulations were run for one year for each location and the annual utility costs, compared to the baseline energy costs and the costs using the sensible storage are shown in Table 7-17, while the comparison of energy use between the ice storage and the sensible storage is shown in Figure 7-12.

Table 7-17: Comparison of energy costs using both sensible and latent storage

\begin{tabular}{lcccc}
\hline Location & $\begin{array}{c}\text { Baseline Energy } \\
\text { Cost }(\$)\end{array}$ & $\begin{array}{c}\text { Energy Cost - } \\
\text { Sensible (\$) }\end{array}$ & $\begin{array}{c}\text { Energy Cost - } \\
\text { Latent (\$) }\end{array}$ & $\begin{array}{c}\text { Difference } \\
\text { Sensible to } \\
\text { Latent (\%) }\end{array}$ \\
\hline Miami & 630.44 & 446.39 & 624.56 & 40.0 \\
Phoenix & 1063.40 & 937.65 & 1059.89 & 13.0 \\
Portland & 1009.48 & 963.93 & 1061.52 & 10.1 \\
Boston & 890.11 & 954.81 & 1199.28 & 25.6 \\
Toronto & 1031.52 & 1104.57 & 1199.79 & 8.6 \\
Sudbury & 1074.24 & 1175.67 & 1286.52 & 9.4 \\
\hline
\end{tabular}




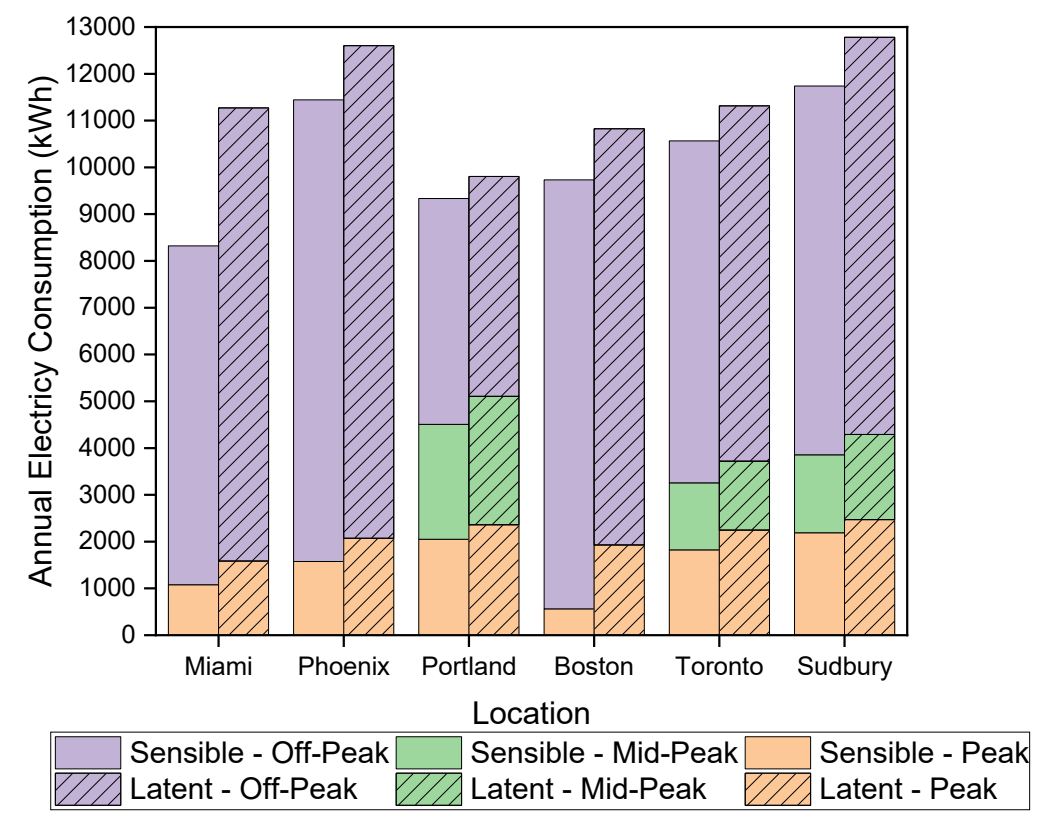

Figure 7-12: Comparison of energy consumption between latent and sensible storage based on time of use

Based on these results, it has been shown that the switch from the optimized sensible storage system using a 50/50 water/glycol solution to the ice storage significantly increased the annual utility consumption and annual utility costs. A number of factors influenced these results, causing the rise in both annual consumption and utility cost increases. In terms of the energy costs, it can be seen that there is an increase in each city for the peak energy consumption, which is most likely caused by a decrease in the total amount of cooling potential being stored. This could be caused by one of two items: a lower capacity in the ice storage when compared to the sensible tank, particularly at higher volumes, or a decrease in the storage set-point as a result of the predictive weather controls. In all cities, the maximum ice storage capacity was set at $35 \mathrm{kWh}$ of cooling potential, meaning that the lower of $35 \mathrm{kWh}$ or the set-point determined through predictive control 
was utilized. To compare this to the capacity of the optimized storage tanks, the storage capacity of each selected system was determined and are listed in Table 7-18.

Table 7-18: Optimal sensible storage capacity by location

\begin{tabular}{lc}
\hline Location & $\begin{array}{c}\text { Optimized Storage Capacity } \\
(\mathrm{kWh})\end{array}$ \\
\hline Miami & 30.4 \\
Phoenix & 26.6 \\
Portland & 40.5 \\
Boston & 22.8 \\
Toronto & 25.3 \\
Sudbury & 15.2 \\
\hline
\end{tabular}

From this, the maximum storage capacity only decreased in Portland, but in all other locations, the maximum storage capacity increased when the switch to ice storage was made. As such, the main impact had to be the set-point determined through the predictive control that is increasing the peak consumption. To test its impact, the simulations were rerun using a static control of $35 \mathrm{kWh}$ for Miami and Phoenix and the annual energy consumption and utility costs are shown in Table 7-19.

Table 7-19: Comparison of static and predictive control

\begin{tabular}{lcccc}
\hline Location & $\begin{array}{c}\text { Energy Cost - } \\
\text { Predictive }(\$)\end{array}$ & $\begin{array}{c}\text { Energy Cost - } \\
\text { Static }(\$)\end{array}$ & $\begin{array}{c}\text { Energy } \\
\text { Consumption - } \\
\text { Predictive }(\mathrm{kWh})\end{array}$ & $\begin{array}{c}\text { Energy } \\
\text { Consumption - } \\
\text { Static (kWh) }\end{array}$ \\
\hline Miami & 624.56 & 603.81 & 11,272 & 11,510 \\
Phoenix & 1059.89 & 1024.27 & 12,600 & 12,727 \\
\hline
\end{tabular}

From this, it can be seen that the predictive control, in these two cities with cooling dominated climates increases the annual utility costs, while also increasing the total consumption. This result is contradictory to the results previously obtained for Ottawa, and 
indicates for cooling dominated climate, the benefits of predictive control, particularly when using ice storage has limited, or is detrimental to the annual utility consumption.

While this accounted for some of the increase, it was not the dominate factor in the annual utility cost increases. This was caused by the significant increase in utility consumption during off-peak periods across all six locations examined. The potential difference between the two systems is the storage efficiency of the thermal storage. In Section 5.3, a comparison was made between the storage efficiency between two systems of the same volume (in that case $270 \mathrm{~L}$ ), but no comparison was made at comparing the two system with different volumes, but similar cooling potential storage. From the results of these simulations, it can be seen that the switch from a sensible storage to latent storage with similar storage capacity significantly increase the amount of electrical input required to charge the system.

To further examine this point, simulations were run with the chiller connected to a sensible cold thermal storage tank with a volume of $1500 \mathrm{~L}$ and $2000 \mathrm{~L}$, and a $500 \mathrm{~L}$ latent 
ice storage tank. The results, comparing the amount of cooling potential stored compared to the electrical input are plotted in Figure 7-13.

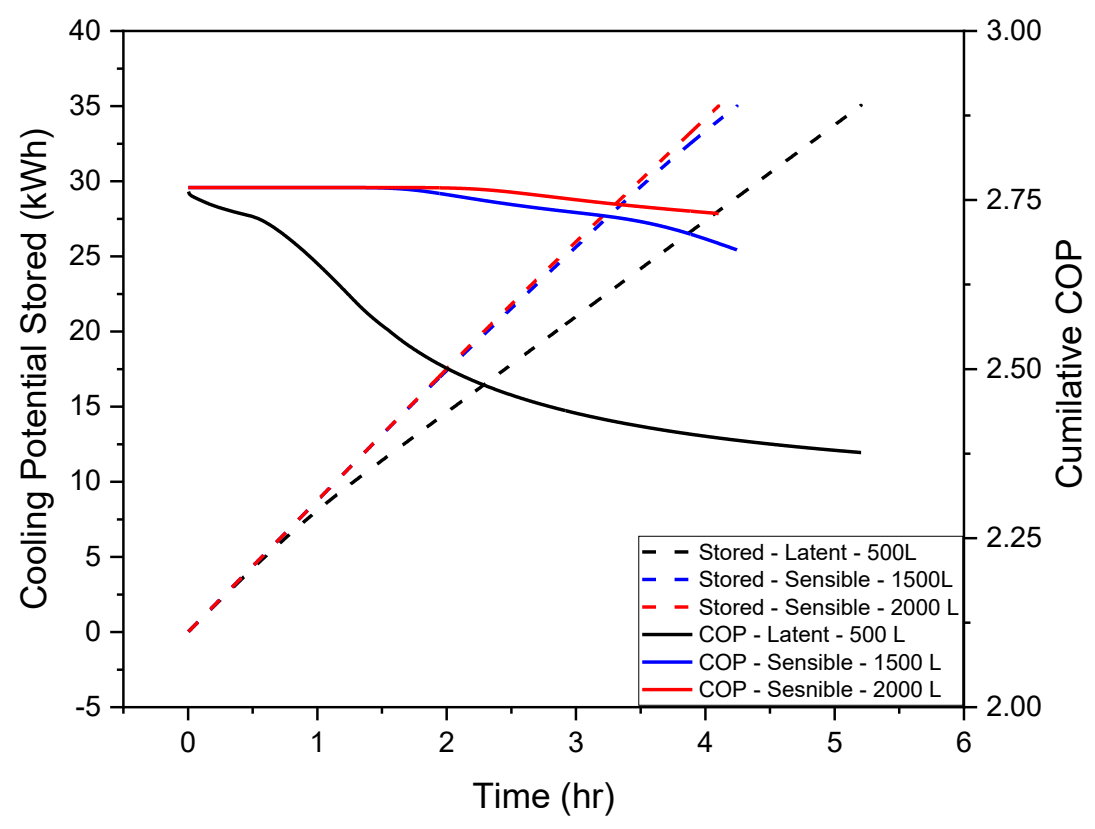

Figure 7-13: Comparison of Charge performance to store the same quantity of cooling potential

Based on the results in this graph, it can be seen that the performance of the chiller degrades much quicker when charging the ice system compared to the sensible storage. This is because the return temperature from the ice storage system drops quickly to below $0^{\circ} \mathrm{C}$, and then becomes approximately steady as it stores cooling potential as ice. This is in comparison to the sensible storage, which decreases much slower, as the much larger volume of the tank is cooled down, and depending on the amount of storage desired and the set-point of the tank, may never go below $0^{\circ} \mathrm{C}$. This shows the trade-off between sensible and latent storage cold storage. Latent storage can achieve much higher storage capacities, allowing systems to be used in tight residential spaces, or as a retrofit, but the use of latent storage reduces the overall performance of the system.

Based on these results, when designing a system, the physical size of the system compared to the performance of the system must always be considered. To improve the 
performance of the latent storage, a storage medium with a solidification point greater than $0^{\circ} \mathrm{C}$, but less than the fluid temperatures required to meet the cooling demand (typically $15^{\circ} \mathrm{C}$ ) should be selected. Some wax type materials have been designed to meet these requirements, however, as the liquid wax cannot be pumped to directly provide the cooling, a second liquid-based piping system is required through the material to provide cooling. Alternatively, the same piping system could be used for both charging and discharging, but in this case, simultaneous charging of the system and cooling of the space could not be realized. These storage mediums are significantly more expensive then the water used in an ice storage system, and the charge/discharge complexities would add additional costs to the system. For these reasons, ice storage was still selected as the latent storage method for this system, but it is important for any application that all potential solutions be explored.

\subsection{Decoupling Heat and Cooling Systems}

Up to this point in this study, the focus has been on developing a single system to meet the both the heating and cooling loads of a building, to reduce the number of components required. This was also done to achieve a secondary goal of electrifying the heating and cooling system, which would require that a heat pump that meets both the space heating and cooling demands. That said, most of Ontario, and in particular in large cities like Ottawa and Toronto, meet most of the space heating load using natural gas. Due to the low cost and abundance of natural gas, the economics are even poorer when looking at shifting from a natural gas furnace to heating with a heat pump coupled with thermal storage. As such, what a more realistic system would look like in Ottawa would be a natural gas furnace with a chiller and thermal storage system to meet the space cooling demand. The upsides to this system should be lower operating costs and being more in line with the 
status quo, while the down side is the use of natural gas which has significantly higher greenhouse gas emissions when compared to the Ontario electrical grid (this is not true for all locations and is depending on the electrical supply source energy).

To determine the potential for using this system to just meet the cooling load in Ottawa, just the energy requirements and the costs to meet the cooling load and the coupled domestic hot water systems were analyzed. In the base case for Ottawa, the total cost for space cooling and domestic hot water using 2020 rates is $\$ 1098$, while the system using the chiller and ice storage, coupled to the hot water system costs $\$ 1135$, which is an increase of $3.3 \%$. Although these values could be determined using the data provided in Section 6.5, the potential of this system is discussed, and examined as a potential system in Section 7.5, which analyzes the lifetime potential for the system.

\subsection{Projected Lifetime Cost Analysis}

The final scenario that will be analyzed is the long-term cost saving potential of this system. Up to this point, the financial analysis only determined the offsetting potential in year 1 (current year). The investment in this system would be realized over many years, with the average heat pump having a median lifespan of 15 years according to the ASHRAE Equipment Life Expectancy Chart, while the lifespan of similar water-based heat pumps increases to 19 years. As such, to truly determine the potential of the system, an analysis over this period would be required.

\subsubsection{Determining Lifetime Utility Costs}

One of the biggest challenges in determining lifecycle costs is determining what the energy costs will be over the length of the equipment life, as the annual increase in electrical cost is driven by political decisions, natural resource costs and the global 
economy. As such two of different models were developed for the annual cost of electricity in Ontario over 15-, 20- and 25-year periods. The first is based strictly on natural inflation. The Bank of Canada sets an inflation goal of $2 \%$ annually through the raising and lower of their interest rates [99]. Although in recent years Canada has not reached 2\% annual inflation, as one of the few firm targets for annual inflation, all electrical prices (peak, midpeak and off-peak rates) were increased each year by $2 \%$ of the preceding years rate, starting with the 2020 rate, including delivery fees and HST. The second method used the historic rate of increase for each electrical tier. Prices reached their lowest point since time of use pricing was introduced in November of 2007 and have increased with an approximately linear trend (with the exception of artificially lowering of prices based on political decisions) [100]. Over the period between November of 2007 and 2019 (when the most recent price increase occurred), an average annual increase for each tier occurred as shown in Table 7-20.

Table 7-20: Rate increases from 2007 to 2019

\begin{tabular}{l|ccc}
\hline Price Tier & 2007 Price (cents/kWh) & 2019 Price (cents/kWh) & $\begin{array}{c}\text { Average Annual Increase } \\
(\%)\end{array}$ \\
\hline Peak & & & 7.5 \\
Mid-Peak & 7 & 20.8 & 6.0 \\
Off-Peak & 3 & 14.1 & 10.6 \\
\hline
\end{tabular}

Using these two rates of increase, the annual utility costs for each of the next 25 years were determined. The electrical rates determined for every $5^{\text {th }}$ year are tabulated in Table 7-21. 
Table 7-21: Potential rate increases over the next 25 years

\begin{tabular}{l|ccc|ccc}
\hline \multirow{2}{*}{ Year } & \multicolumn{3}{c|}{ 2\% Inflation } & \multicolumn{3}{c}{ Historic Increase } \\
\cline { 2 - 7 } & Peak & Mid-Peak & $\begin{array}{c}\text { Off- } \\
\text { Peak }\end{array}$ & Peak & Mid-Peak & $\begin{array}{c}\text { Off- } \\
\text { Peak }\end{array}$ \\
\hline 2020 & 25.17 & 17.93 & 13.07 & 25.17 & 17.93 & 13.07 \\
2025 & 27.79 & 19.80 & 14.43 & 36.13 & 23.99 & 21.63 \\
2030 & 30.68 & 21.86 & 15.93 & 51.88 & 32.11 & 35.80 \\
2035 & 33.88 & 24.13 & 17.59 & 74.47 & 42.97 & 59.24 \\
2040 & 37.40 & 26.64 & 19.42 & 106.92 & 57.50 & 98.03 \\
2045 & 41.29 & 29.42 & 21.44 & 153.50 & 76.95 & 162.24 \\
\hline
\end{tabular}

From this table, it can be seen that the strategy using the historic increase over the past 12 years caused a problem where off-peak rates end greater than peak rates. This was caused by the use of compounding rate increases, and the fact that over the past 12 years, off peak rates have risen at a rate which is much higher rate than peak periods. This pricing structure is not realistic as the entire objective of time of use billing is to incentivize the use of off-peak electricity. If the off-peak rates were higher, this would flip the incentive to use more electricity during peak periods, and as such, this rate structure was deemed not valid and was not used to determine the lifecycle costs of the system. Additionally, Ontario has experienced one of its most rapid increases of electricity costs in its history over the past decade, and as such, rates back to 1995 (25 years) were examined. From 1995 to 2007, when time of use rates were introduced, utility rates rose by approximately $25 \%$ [101]. Since time of use billing has been introduced, the blended rate, which is the average price the consumer pays across all times, have further increased by $150 \%$. As such, over the last 25 years, the actual cost of electricity in Ontario has increased $212.5 \%$. To factor in the recent increase in cost, as well as the requirement for new generation in Ontario, an increase in the next 25 years of $250 \%$ to $350 \%$ is realistic, but the $\sim 1000 \%$ increase that would occur 
from a $10 \%$ annual increase seen over the last 12 years is not realistic to occur continuously for the next 25 years.

Since the straight increase based on historic rates did not give a valid solution, two potential rate increase schemes were developed using the data obtained over the past 25 years. The first is assuming that the total increase over the next 25 years is $300 \%$, which works out to an annual price increase of $5.7 \%$. The second- and third-rate increases assumed a government strategy of trying to encourage off-peak consumption, and increased the off-peak rate by that of inflation ( $2 \%)$, while peak periods increased at a rate of $5.7 \%$ and $8 \%$ The utility costs per kilowatt hour are for the three new rate increase schemes are shown in Table 7-22.

Table 7-22: Projected utility rates over the next 25 years with different increase scenarios

\begin{tabular}{|c|c|c|c|c|c|c|c|c|c|}
\hline \multirow[t]{2}{*}{ Year } & \multicolumn{3}{|c|}{$5.7 \%$ Inflation } & \multicolumn{3}{|c|}{$\begin{array}{l}\text { 2\% Off-Peak, } \\
5.7 \% \text { Peak }\end{array}$} & \multicolumn{3}{|c|}{$\begin{array}{l}\text { 2\% Off-Peak, } \\
8 \% \text { Peak }\end{array}$} \\
\hline & Peak & $\begin{array}{l}\text { Mid- } \\
\text { Peak }\end{array}$ & $\begin{array}{l}\text { Off- } \\
\text { Peak }\end{array}$ & Peak & $\begin{array}{l}\text { Mid- } \\
\text { Peak }\end{array}$ & $\begin{array}{l}\text { Off- } \\
\text { Peak }\end{array}$ & Peak & $\begin{array}{l}\text { Mid- } \\
\text { Peak }\end{array}$ & $\begin{array}{l}\text { Off- } \\
\text { Peak }\end{array}$ \\
\hline 2020 & 25.17 & 17.93 & 13.07 & 25.17 & 17.93 & 13.07 & 25.17 & 17.93 & 13.07 \\
\hline 2025 & 33.21 & 23.66 & 17.24 & 33.21 & 23.66 & 14.43 & 36.98 & 26.35 & 14.43 \\
\hline 2030 & 43.82 & 31.21 & 22.75 & 43.82 & 31.21 & 15.93 & 54.34 & 38.71 & 15.93 \\
\hline 2035 & 57.81 & 41.18 & 30.02 & 57.81 & 41.18 & 17.59 & 79.84 & 56.88 & 17.59 \\
\hline 2040 & 76.28 & 54.34 & 39.61 & 76.28 & 54.34 & 19.42 & 117.32 & 83.57 & 19.42 \\
\hline 2045 & 100.64 & 71.69 & 52.26 & 100.64 & 71.69 & 21.44 & 172.38 & 122.79 & 21.44 \\
\hline
\end{tabular}

\subsubsection{Calculating Lifetime Utility Costs}

Once the potential utility cost structure for the next 25 years was determined for Ottawa, the next step was to calculate the annual utility costs for each of the next 15-, 20-, and 25-years. This corresponds with the expected range of the lifespan of a heat pump, with 15-20 being the median life depending on exact specifications, while 25 -years would be a unit that lasts slightly longer then the median lifespan of the units and provides the upper bounds of potential performance. The first step was determining the expected utility 
costs for the base case using a heat pump to directly heat and cool the space, and an electric resistance hot water system. The results for annual and cumulative utility costs for each of the predict price models are shown in Table 7-23, in five-year increments.

Table 7-23: Annual and total utility costs for the base system over the next 25 years (all values in \$)

\begin{tabular}{l|llllllll}
\hline & \multicolumn{2}{|c}{$2 \%$ Inflation } & \multicolumn{2}{c}{$5.7 \%$ Inflation } & \multicolumn{2}{c}{$\begin{array}{c}\text { \% Off-Peak, } \\
5.7 \% \text { Peak }\end{array}$} & $\begin{array}{r}2 \% \text { Off-Peak, } \\
8 \% \text { Peak }\end{array}$ \\
\cline { 2 - 9 } Year & Annual & Total & Annual & Total & Annual & Total & Annual & Total \\
2020 & 1,655 & 1,655 & 1,655 & 1,655 & 1,655 & 1,655 & 1,655 & 1,655 \\
2025 & 1,827 & 10,437 & 2,183 & 11,454 & 2,026 & 11,007 & 2,165 & 11,389 \\
2030 & 2,017 & 20,134 & 2,880 & 24,384 & 2,500 & 22,514 & 2,888 & 24,282 \\
2035 & 2,227 & 30,840 & 3,800 & 41,443 & 3,108 & 36,777 & 3,919 & 41,666 \\
2040 & 2,459 & 42,660 & 5,014 & 63,951 & 3,890 & 54,582 & 5,400 & 65,489 \\
2045 & 2,714 & 55,710 & 6,615 & 93,647 & 4,899 & 76,954 & 7,540 & 98,593 \\
\hline
\end{tabular}

Once the baseline was determined, the annual and total electricity costs were calculated over the same period for the system utilizing the chiller and ice-based cold thermal storage. These results were plotted and compared to the baseline, with the periods of interest highlighted in Figure 7-14while the cumulative was plotted in Figure 7-15.

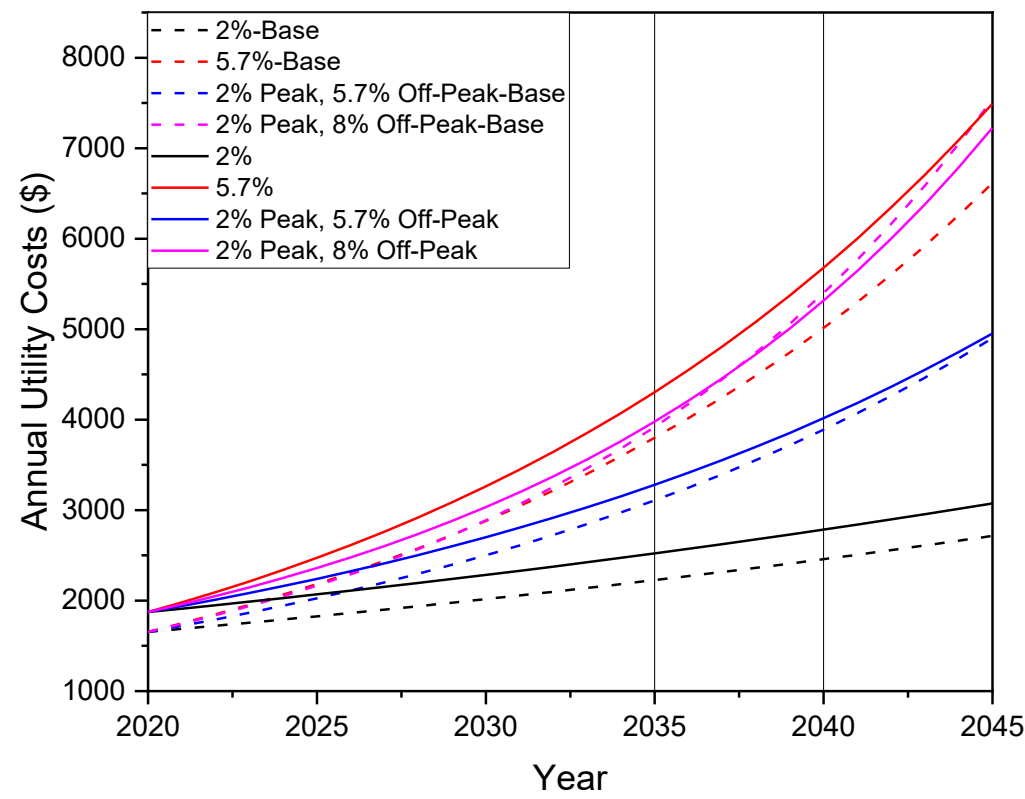

Figure 7-14: Comparison of annual utility costs per year for the next 25 years 


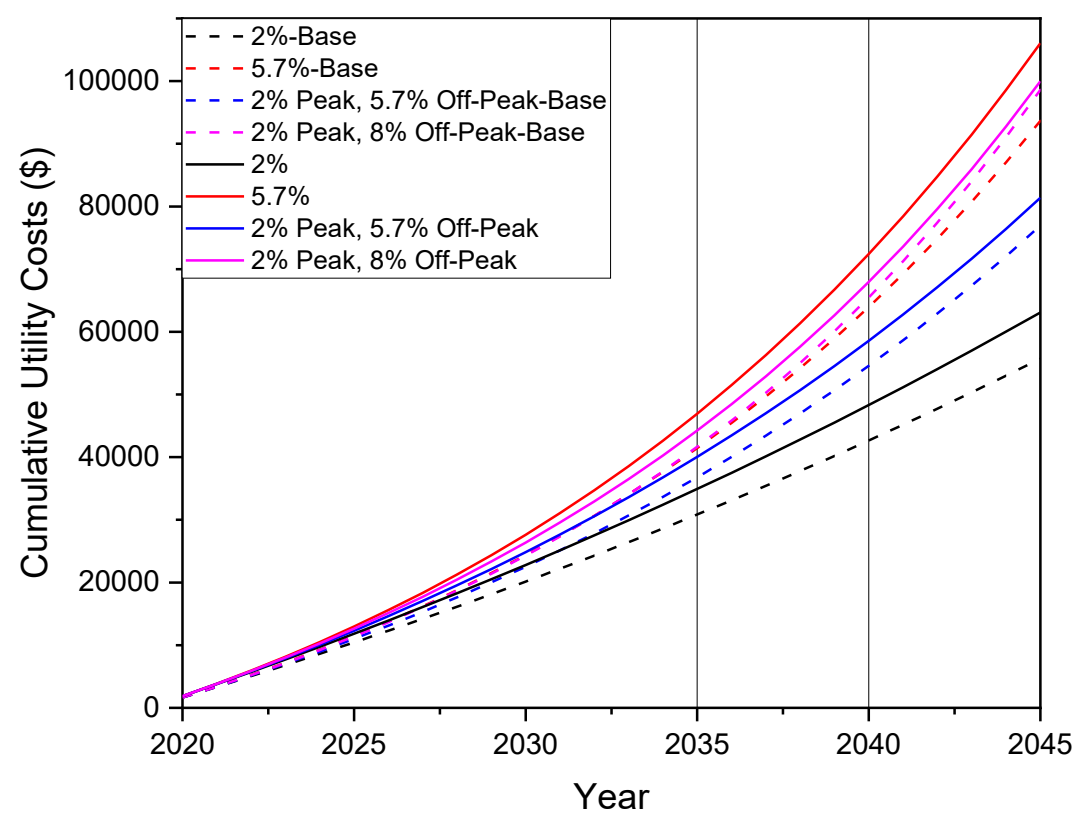

Figure 7-15: Comparison of total utility costs over the next 25 years

From these two graphs, a number of conclusions can be determined. In terms of annual utility costs, in all price increase scenarios, the base case is cheaper to operate for the first 17 years. Starting in the $18^{\text {th }}$ year (2038), if pricing were to increase at $2 \%$ offpeak, $8 \%$ peak, the system using the chiller and the ice storage becomes more economical on an annual basis. No other price increase scenario examined would allow the annual utility costs of the system using thermal storage to be lower than the base scenario in the potential 25-year lifespan of the system.

When looking at the total operating costs and comparing the base case to the chiller and thermal storage system, in all cases, and for all 3 outlook periods (15-, 20-, and 25years), the base case always has lower operating costs when compared to the chiller and thermal storage system. This includes the $2 \%$ off-peak, and $8 \%$ peak increase, which in the final years has a lower annual operating cost. As discussed previously, the savings from the final 8-years does not overcome the deficit created in the first 17-years. Based on this analysis, which has not even looked at the initial increased capital costs, the single system 
using thermal storage to meet the heating, cooling and DHW loads cannot be economically viable over the lifespan of the system.

To further determine the long-term financial viability of the chiller coupled with the ice storage, instead of looking at all building loads, the system was examined to meet just the cooling and DHW loads. In this case, a regular natural gas furnace would be used for space heating, and is not included in this analysis. The same rate increases were utilized as those for the system meeting all building loads, and the annual and cumulative energy was calculated and compared between the base case and the chiller thermal storage system. The annual cost for both systems are plotted in Figure 7-16, while the cumulative costs are plotted for each system in Figure 7-17.

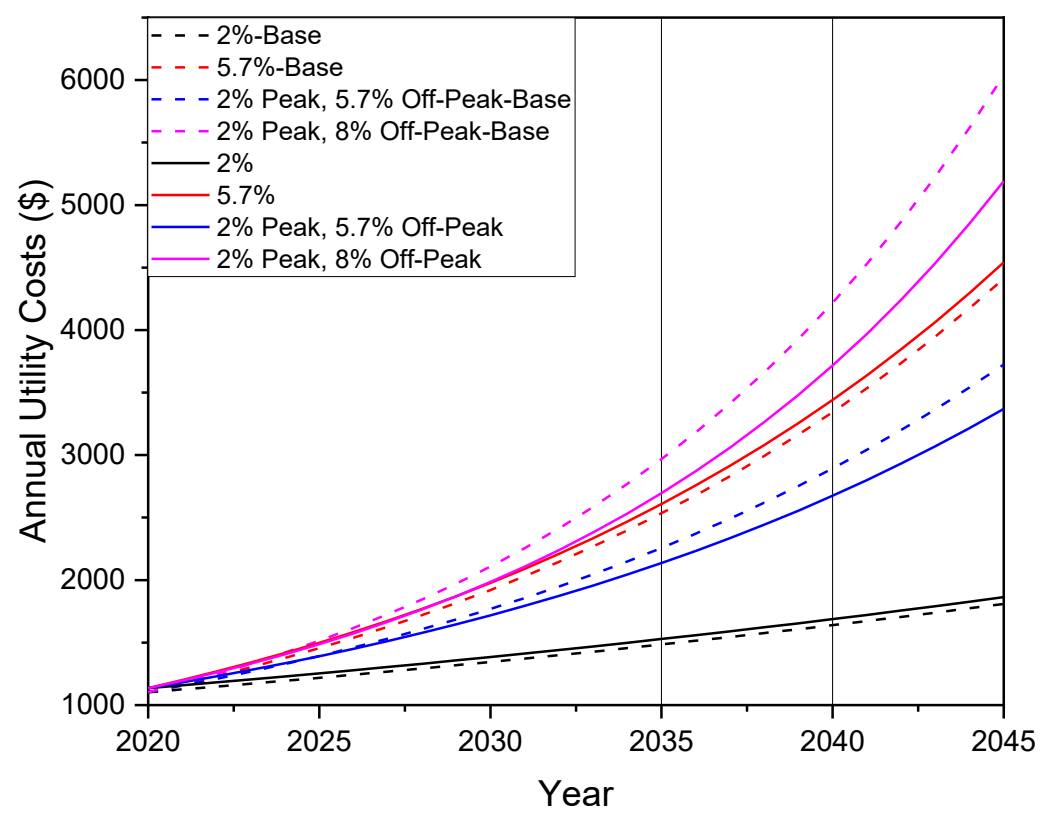

Figure 7-16: Annual utility costs to meet cooling and DHW loads for the next 25 years 


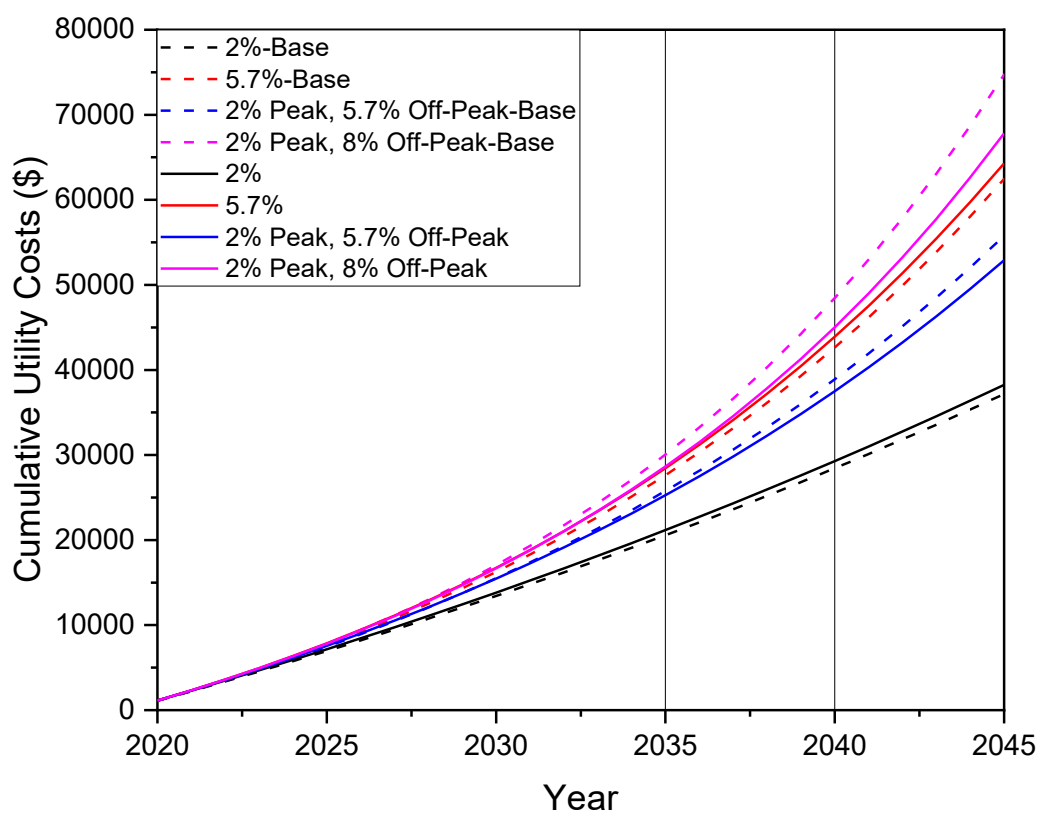

Figure 7-17: Total cost to meet space cooling and DHW loads over the next 25 years

When looking at the annual utility costs to meet the cooling and DHW load, the annual utility costs with the chiller and thermal storage system are lower than the base case early on in the lifespan where the peak rate increases quicker than the off-peak rate. When the peak rate increases by $5.7 \%$ this cross-over occurs in year 6 , while when the peak rate increases at $8 \%$, occurs in year 4 . When a constant inflation is used, the base case always remains lower cost than the system using the chiller and thermal storage system. When these costs are calculated over the 15-, 20-, and 25-year potential lifespans, the operating costs and net difference for each system were calculated as shown in Table 7-24. 
Table 7-24: Comparison between total utility costs to meet space cooling and DHW loads over the next 25 years - All values in Canadian Dollars

\begin{tabular}{l|cccccc}
\hline & \multicolumn{3}{|c}{$2 \%$ Off-Peak, 5.7\% Peak } & \multicolumn{3}{c}{$2 \%$ Off-Peak, 8\% Peak } \\
\cline { 2 - 7 } & Base & $\begin{array}{l}\text { Chiller and } \\
\text { Storage }\end{array}$ & Difference & Base & $\begin{array}{l}\text { Chiller and } \\
\text { Storage }\end{array}$ & Difference \\
\hline $\begin{array}{l}2035 \\
(15 \text { Years })\end{array}$ & 25,769 & 25,264 & -505 & 30,053 & 28,635 & -1418 \\
$\begin{array}{l}2040 \\
(20 \text { Years })\end{array}$ & 38,896 & 37,505 & -1391 & 48,454 & 45,026 & -3428 \\
$\begin{array}{l}2045 \\
(25 \text { years })\end{array}$ & 55,768 & 52,892 & -2876 & 74,731 & 67,814 & -6917 \\
\hline
\end{tabular}

From these values, it can be seen that there is the potential for a net reduction in utility costs over the lifespan of the installed system, and as such, there is the potential for economic benefit using the chiller and thermal storage system. This section only examined the potential in reduction of the total operating costs, but the complete lifecycle costs must be considered, including the initial investment in the more complex system to determine its true economical potential.

\subsubsection{Complete Lifecycle Analysis}

Once the annual utility rates over the projected lifespan of the units were determined, the final step for completing the lifecycle cost analysis was to calculate the incremental cost of the installation of the system. The incremental costs include the increased cost of a medium temperature chiller compared to a standard air conditioner, the cost of the ice thermal storage system, the installation of a $150 \mathrm{~L}$ preheat hot water tank, and the added costs of extra pumps and control systems. As this is not a commercially available system, direct pricing is not available and as such, some assumptions needed to be made to determine what the costs would be if the system became commercially available and viable. At the current time, a high-end air-conditioner with performance similar to what was modelled, and at a comparable size (3.5 tons of cooling) costs approximately $\$ 4500$ [102], whereas 
the unit tested in the experimental setup costs approximately $\$ 12,000$ installed. This was a custom unit to meet the flow and temperature conditions required for this study, and as such had a higher cost then a commercially produced unit. There is no straight rule for the decrease in cost of a commercial unit as opposed to a custom unit, and as such, a $30 \%$ decrease in cost was initially assumed when looking at the costs of other heat pump and chiller units of similar size, if the system was to be commercially produced as a unit.

When looking at the ice storage system, there is no data available to guide the fabrication costs of this system. Any available system is for commercial applications and is many magnitudes greater in size, and as such not scalable. As such, an initial estimate of $\$ 3000$ was assumed. To account for the added costs of the preheat, a small hot water tank had to be installed. Including installation, this added tank would cost approximately $\$ 1000$. Hot water tanks typically only last $12-15$ years, and as such, a second $\$ 1000$ cost, indexed to year 15 must be included in the lifecycle cost of the system. Finally, the complete system requires additional pumps, control valves and a much more complex control strategy. To compensate for these costs, an added premium of $\$ 3000$ was assumed. Based on all of the added costs, it was assumed that the initial system premium would be approximately $\$ 10,500$, plus an additional $\$ 1000$ cost during year 15 , and the system must overcome this initial cost to be economically viable.

It is not a fair comparison to simply look at the net annual cost savings over the period it is being examined over compared to the increase in the initial capital cost. This is because the initial expenditure represents a lost economic opportunity, which would be incurring interest over the period of study. If a conservative net annual gain of $2 \%$ annually (the expected rate of inflation) is assumed, the actual cost of that initial investment in the 
system at 15-, 20-, and 25-years respectively is $\$ 14,131, \$ 17,087$ and $\$ 18,866$. This value was then compared to the total energy costs, and a net operating cost for the system after 15-, 20- and 25-years, and these results are shown in Table 7-25.

Table 7-25: Lifecycle cost analysis using two different rate structures - All values in Canadian Dollars

\begin{tabular}{l|cccccc}
\hline & \multicolumn{3}{|c}{$2 \%$ Off-Peak, 5.7\% Peak } & \multicolumn{3}{c}{ 2\% Off-Peak, 8\% Peak } \\
\cline { 2 - 7 } & $\begin{array}{l}\text { Energy } \\
\text { Savings }\end{array}$ & $\begin{array}{c}\text { Initial } \\
\text { Capital }\end{array}$ & Net Cost & $\begin{array}{c}\text { Energy } \\
\text { Savings }\end{array}$ & $\begin{array}{c}\text { Initial } \\
\text { Capital }\end{array}$ & Net Cost \\
\hline $\begin{array}{l}2035 \\
(15 \text { Years })\end{array}$ & -505 & 14,131 & 13,626 & -1418 & 14,131 & 12,713 \\
$\begin{array}{l}2040 \\
(20 \text { Years })\end{array}$ & -1391 & 17,087 & 15,689 & -3428 & 17,087 & 13,659 \\
$\begin{array}{l}2045 \\
(25 \text { years })\end{array}$ & -2876 & 18,866 & 15,990 & -6917 & 18,866 & 11,949 \\
\hline
\end{tabular}

Based on these calculations and the underlying assumptions, the system is not close to being economically viable when examining the lifecycle costs of the system. As such, to make the system economically viable, one of three factors must occur. These are 1) the initial cost of the system decreases, 2) the average annual increase in peak electrical rates is greater than the assumed $8 \%$ or 3 ) government or utility rebates and incentives are provided to offset a portion of the initial capital costs.

\subsubsection{Potential for Incentive Programs}

To determine what each of these three possible solutions would need to look like to be economically viable, each was calculated to determine the required change to have the system break even after 25 years. For the decrease in capital cost and government incentives, the calculations were based on the 25-year life expectancy, and $8 \%$ peak electrical rate increase. When looking at the initial capital costs, the total savings over the 25 years of $\$ 6917$ must be discounted back to year 0 , and using the discount rate of $2 \%$, 
this allows for a $\$ 4216$ initial investment, or about $36 \%$ of the initial estimate for the incremental cost of the system. The only potential way to reach this incremental cost would be the development of a prefabricated packaged system incorporating all the components, and thus significantly reducing the installation costs of the system.

When examining government incentives, two different strategies could be employed. The first would be to provide an initial rebate at the time of purchase, which, to allow the homeowner to break-even over 25 years, would amount to a rebate a little more than $\$ 6000$. The other incentive program that could be implemented would be an annual rebate on electrical costs as a repayment for shifting consumption from peak to off-peak periods and as a result contributing to the flattening of the overall electrical consumption on the grid. To determine what that annual rebate would be, the future value of annual payments had to be calculated. If the repayments were to be made across all 25 years, an annual payment from the government or utility provider of $\$ 350 /$ year would be required to offset the increased capital costs, when factoring in the interest paid on the outstanding initial costs. Alternatively, a fixed, quicker payback period could be employed by the utility provider, and if the annual payments only occurred in the first 15 years after the initial installation, an annual rebate to the homeowner would need to be $\$ 450 /$ year to offset the increased initial capital costs.

The final change that could be made to make the system economically viable would be if there is a larger annual average increase in peak electrical rates than the assumed $5.67 \%$ and $8 \%$. If the off-peak rate increase stays at inflation of $2 \%$, the peak increase rate to create a net savings of $\$ 18,866$ needs to be found. When solving through, an annual increase of $11.9 \%$ for peak rates is required, at which point, the total base electrical costs 
for cooling and DHW would be $\$ 129,918$, while the integrated chiller and thermal storage would have a total utility cost of $\$ 111,246$. The net savings in this case would be $\$ 18,675$, which would directly offset the initial investment and the loss of potential interest over the 25 -year period. From this analysis, in Ontario, the only way this system would ever become economically feasible is through government incentives, be it through the support of developing the system and supporting the commercialization, through incentive or rebate programs, or by changing the pricing structure in which the peaks rates increase at a much greater rate than the off-peak rates.

\subsection{Conclusions on Potential for the Integrated System}

This chapter reviewed the impact of the assumptions made during the modelling of the systems, the impact of the electrical rate structure, the potential for implementation of the systems in other North American locations and finally, the lifecycle costs of the systems. From these results, it was first shown that the modelling assumptions, primarily the source side heat input during the heating season, did impact the overall annual performance of the system. Although the annual performance was impacted, the change in performance was approximately proportional between the base case and the system using the chiller and thermal storage. As such, these assumptions did not have a significant impact on determining the potential for demand side management using a medium temperature chiller and cold thermal storage, be it sensible or latent.

Next the impact of the utility rates, including the changes in the past 4 years, as well as the potential for using market rates was examined. The impact of rates changes over the course of this project were negligible, as the increases were proportional across all rate tiers. Had there been a relative change between rate structures, this would have had 
an impact on the potential for the system; this did not occur over recent years, but formed the basis for predicting future rate increases. Finally, the impact of changing from fixed rates, currently utilized for residential customers, to market rates, utilized for large commercial customers was analyzed. Market rates had a large fluctuation from year to year, and when compared to fixed rates, were sometimes favorable to the chiller and thermal storage system, while other year's rates were detrimental. The use of market rates introduces a large unknown into the annual cost calculations, and in predicting the lifespan cost of the system. Depending on the year and in particular, that year's weather conditions, the switch to market rates could reduce the annual electrical costs. One thing that was not explored, as the weather contained within a CWEC file cannot be correlated to a specific market cost, is the use of a control logic that correlates the tank set-point to the current market cost of electricity. This would allow very low and negative rates to be exploited but would require real-time feedback from the electrical grid and the market rates. This could produce excellent returns but would significantly increase the complexity of the control system and would require the development of a predictive control model, to both predict the amount of heating and/or cooling required, as well as determine and predict future electrical rates to determine the optimal thermal storage charge strategy.

The same house and system was modelled in multiple cities across North America which use time of use billing plans to determine if different locations and rate structure influence the economic potential of the system. These included one location in each of the seven ASHRAE climate zones. It was found in southern, cooling dominated climates, and in particular locations with a high peak to off-peak pricing ratio, significant energy cost savings approaching $30 \%$ can be realized using cold sensible thermal storage. To achieve 
this, large storage volumes between $1500 \mathrm{~L}$ and $2000 \mathrm{~L}$ were required, but when switching to ice storage, and its $500 \mathrm{~L}$ volume, there was a significant performance degradation, and costs returned to approximately the same as the base case. This showed that although a sensible and latent thermal storage system with similar volumes have similar charge rates and performance, the same cannot be said for two storages that have the same total storage capacity. In this case, the charge efficiency is much higher in larger volume thermal storage tanks compared to lower volume latent ice storage systems, and this must be taken into account when deciding between the two systems.

Finally, the lifecycle costs of the system were determined to see if over its expected lifetime, the system will allow for savings in overall utility costs based on different rate increase predictions. It was found that based on all of the tested predictive cost models, when factoring in the initial cost of the system, it is only economically feasible if government incentives or rebates are introduced, or the initial capital costs were to be significantly reduced. Looking only at the energy costs, if peak rates increased at a pace greater than those of off-peak periods, over a 25 year lifespan of the system, between $\$ 2800$ and $\$ 6900$ reduction could be realized, but the initial capital outlay is significantly greater than that, in both hard costs and lost opportunity costs. 


\section{Chapter 8: Conclusions and Future Work}

The overall objective of this research project was to determine whether the use of a heat pump system coupled with thermal storage system(s) could be used to shift electrical consumption from peak to off-peak periods. This demand side management strategy would allow the homeowner to shift their consumption, while not having to impact their comfort by making large changes to their home thermostat set-points. This work started by first examining the performance and developing validated models of each of the main components, including a standard heat pump, medium temperature chiller, cold sensible thermal storage and a latent cold storage using ice as the storage medium. The standard thermal storage models used for hot water accurately predict the stratification within cold storage tanks, and their accuracy improves when a glycol/water solution is used compared to pure water. The ice storage model was developed using fundamental heat transfer equations and validated on a small-scale ice storage experimental set-up. These models were then used to determine the potential for demand side management.

\subsection{Integration of a heat pump and thermal storage}

The interaction between the thermal storage and heat pump/chiller was compared both experimentally and using the validated TRNSYS models. One of the first underlying questions of this work was whether the use of a stratified cold thermal storage tanks could improve the charge efficiency of the system. The supply temperature to the evaporator of the heat pump/chiller would be higher when using a stratified tank but would need to operate at lower flow rates. Through both experimental and simulated tests, this was proven untrue, as the decrease in performance from the lower flow rates outweighed the improved performance from the higher temperatures. This dictated that for the rest of the study, high 
flow rates were utilized to improve the system performance. The higher flow rates also were shown to improve the heat transfer into the ice storage system, and as such, the same amount of cooling can be stored with a smaller number of heat transfer pipes.

A second interaction between the heat pump/chiller and the thermal storage systems that was conducted was to compare the difference in charge efficiency between the sensible thermal storage and the latent ice storage. The study looked at the charge efficiency of the two systems if the same tank volume was used. In this case, it was shown that the charge efficiency of the sensible storage was slightly larger than the latent system, up to the point where ice formation begins in the latent tank and the return temperatures stop decreasing. The sensible storage stops charging due to low return temperatures much quicker than the ice storage, and in total, the ice storage for the same volume was found to be able to store more than twice that of the sensible storage. This is critical for applications in which space is limited and would work well in retrofit systems, where the tank needs to be installed within an existing space. The second comparison was when the same storage capacity, but different tank volumes were examined. In this case, the charge efficiency in the ice storage tank was significantly lower than the sensible tank, as the sensible tank has a much lower rate of decrease in temperature.

From these two cases, the importance of selecting the correct storage method and tank volume are critical to the success of the system. If the volume and space the system is to occupy is available, and the storage tanks can be installed in a place with sufficient access, the long term performance and lifecycle costs are much better when using a large sensible storage tank when compared to a smaller ice storage system with the same storage capacity. The ice storage has its benefits in terms of smaller footprint, and much higher 
storage densities, but should only be used when a sensible solution would not be technically feasible, or where other benefits for using ice could be derived, which would be cases where sensible storage would need to reach very low temperatures to meet the storage demands.

\subsection{Annual results and potential for demand side management}

Once the individual components were modelled, and their interaction with a focus on their charge efficiencies was determined, the system as a whole, modelled within a house in Ottawa was examined. The system was slowly built up, adding additional components to determine how each additional component or sub-system impacted annual performance and utility costs. The first iteration used a standard heat pump with both hot and cold thermal storage, but using sensible storage required very large tank volumes. This was caused by the fact the standard heat pump would only function to inlet temperatures of between $0^{\circ} \mathrm{C}$ and $5^{\circ} \mathrm{C}$, and at these temperatures the performance was very poor (COPs at or below 1 for cooling). These results showed that for the system to function and be feasible, lower tank volumes and performance at lower temperatures would be required.

To improve the performance of the system, the standard liquid to liquid heat pump was swapped out for a medium temperature chiller. This chiller was designed and optimized in terms of heat exchanger sizing and refrigerant type to operate effectively with evaporator temperatures as low as $-10^{\circ} \mathrm{C}$. This allowed for both lower sensible storage temperatures using a glycol solution to be achieved (and consequently higher storage densities), and the integration with ice storage. Through annual modelling, it was shown that this chiller was better able to store cooling potential, but because it has been optimized for lower temperatures, it had much lower performance when providing the space heating 
for the space. This increase in space heating demand offsets many of the gains achieved through successfully shifting the peak loads to off-peak periods. This conclusion is valid based on the current circumstances and grid conditions, but could change as grid conditions evolve over time. For instance, as a greater emphasis is being placed on more renewable sources, a shift towards electric heating sources is taking place. As the increase in heating using electrical sources increase, the overnight demand would increase, which could cause a shift in the peak periods on the grid during the winter months. As such, a greater portion of the heating load would occur during mid or peak periods, and therefore could impact the conclusions obtained, and make the system more viable for heating purposes. When looking at only the domestic hot water demands and cooling loads, there is still a small increase annual operating costs when examining the potential for the system in Ottawa. To increase the potential for the system, a larger difference in the cost between peak rates and off-peak rates would be required.

As discussed, the overall goal of this research project was determining whether, through the offsetting of peak loads using thermal storage and a heat pump, a reduction in cost and greenhouse gas emissions could be achieved. Through most of this project a focus has been placed on determining the economic feasibility of these systems, as it was quickly determined that there was not a single scenario in which the greenhouse gas emissions could be reduced in Ontario. Ontario has a relatively clean utility grid with the removal of coal fired plants in the early 2000s and the integration of additional renewable generating sources. Ontario still uses natural gas plants to meet its peak demand, but this constitutes a small portion of the overall production in the province, and as such, there is only a small difference in the amount of greenhouse gases released per kilowatt hour of electricity 
produced. Additionally, unlike utility rates which are constantly rising, the greenhouse gas emissions from electrical production continue to fall. As such, even if the shifting from peak to off-peak electrical consumption resulted in a decrease in natural gas in year one, as generation continues towards more renewable sources, the absolute amount of, and the difference between rate periods will continue to decrease. For these reasons, the goal of reducing the greenhouse gas emissions using demand side management was determined both unachievable and unlikely to have a significant impact on global greenhouse gas emissions due to the low emission rate of Ontario electricity.

As the annual electricity costs increase the utility costs compared to the base case, the lifecycle costs were calculated to determine whether future utility costs increase would make the system economically viable over its life. This was done for the system using the medium temperature chiller and ice storage to meet all building loads, as well as using the system to meet only the space cooling and hot water loads. Through a potential 25 -year lifespan of the system, there remained an increase in utility costs when space heating, space cooling and domestic hot water were considered. A second set of calculations were run, looking only at hot water and space cooling loads, and allowing the space heating to be met using natural gas (as is typical in Ontario). Using this configuration, it was found that depending on how future rate increases are projected, cost savings could be realized compared to the base cooling and domestic hot water loads. The key to these savings was a larger increase in peak rates compared to off-peak rates, and if a rate increase tied to 
inflation ( $2 \%$ annually), and an $8 \%$ increase in peak rates, a savings of almost $\$ 7000$ over the 25 year lifespan in utility costs could be realized.

Although it was found utility cost savings over a 25 -year period could be realized, the total life costs need to be considered. Most notably, this needs to include both the initial capital costs, and the lost opportunity cost of this increase in initial capital cost. Factoring this in, it was found that in all cases, the economics do not work for switching to a system that shifts peak loads to off-peak periods. The only way this system would work is if it became either a government or utility provider priority to reduce the peak loads being placed on the utility grid. If this became a priority, and incentive programs were introduced, either in the form of lump sum payments or annual rebates, the systems could become economically viable, and reduce the peak demand on the grid.

As part of this research program, a single, integrated system was envisioned, under the purview that multiple loads could be met simultaneously using the two sides of the heat pump. For example, this could be using the waste heat produced to meet the cooling load to be either stored for potential nighttime heating, or to meet the domestic hot water load. Within this study, the potential for simultaneous heating/water heating and cooling was examined, and it was found that only one of heating or cooling could be stored simultaneously, without causing a significant degradation in heat pump performance. The performance of the heat pump/chiller decreases with both the increase in condenser temperature and the decrease in evaporator temperature. As such, an attempt to simultaneously charge a hot and cold storage causes more than a twice the rate of decrease in performance compared to charging only one system at a time. Additionally, when looking at attempting to meet the hot water demand, which would have otherwise been met 
using a resistance heater, a drop in COP of more than 1 to meet the cooling load becomes detrimental compared to the directly heating the hot water. From these results, it was found that if storage was occurring, the waste heat or cooling potential could be used directly with limited impact on the performance of the storage system. For example, during the shoulder seasons while the cold thermal storage was charging, if a call for heat was initiated by the thermostat (cold evenings, warm days), the waste heat from charging could effectively meet the demand with limited impact on the overall performance. This is because the return temperatures remained relatively low $\left(20-25^{\circ} \mathrm{C}\right)$ from the air handler, and therefore this was not detrimental to the cooling performance. On the other hand, when the hot water preheating was considered, as the temperature the water is being preheated to increased, the greater the benefit in terms of reducing the direct energy input to meet the DHW demand, but the more energy required to meet the cooling demand. As such, it was found the preheat set-points in excess of $30^{\circ} \mathrm{C}$ would actually increase the annual consumption as the hot water savings is less than the increase in the required energy to meet the cooling load. The lessons learned from this study is that it is not a guarantee that the use of waste heat is always beneficial, and in many cases, the higher or lower temperature created as a result could have a more significant detrimental effect then the benefit derived from the use of the waste heat.

After exploring all options and potential scenarios and rate structures for the system in Ottawa, it was determined that the system is not economically viable under any configuration. The only way to make the system work in Ottawa would be with significant government incentives or rebates or a significant increase between the peak and off-peak rates. Once this was determined, the next question was whether there were any locations 
in North America, using time of use billing, in which the system could be economically viable. Using 2017 utility rates for each city examined, it was found that cooling dominated climates and those with significant peak rate to off-peak rate ratios could see significant savings (up to $30 \%$ ) on annual utility costs if a sensible storage tank with a significant volume (between $1500 \mathrm{~L}$ and $2000 \mathrm{~L}$ ) was utilized. When the system was switched to use an ice storage tank instead of a sensible storage, many of the financial gains were eliminated, but the net cost compared to the base scenario were comparable or lower in these same locations. When looking at climates with a mix of heating and cooling loads, or locations where the difference in peak to off-peak rates were small, the introduction of any type of thermal storage had a net increase in utility costs on an annual basis. As such, each location must be assessed for both its total cooling and heating loads, the proportion of those loads during peak periods, and the utility rate structure to determine whether there is potential for implementing demand side management through a chiller and thermal storage system.

In conclusion, through this work, a number of key findings were found in terms of both the modelling and performance of heat pumps and cold thermal storage, and the potential for their use in to achieve demand side management. From a technical standpoint, the developed system can shift a significant proportion of the peak load to off-peak, and even $100 \%$ of the cooling load if a large enough cold storage is utilized. This could provide significant benefits to the electrical grid if larger implementation rate is realized, which could reduce the required generation and transmission capacities required within the electrical grid. This could lead to a reduction in required capital costs for the utility providers but would come at the cost of the consumer. In every explored configuration, 
over the life of the system, the homeowner would see a significant increase in their costs, and as such, if this is of interest to governments or utility providers, incentive or rebate programs would need to be developed to support homeowners making this change. Learning from other locations throughout North America, a large ratio between peak and off-peak utility rates (approaching 5), would make the economics far more attractive and could allow implementation without the need of government subsidies. Finally, although the benefits are limited if installed in Ottawa, there is more potential for cooling dominated climates, where the benefits could be realized all year round.

\subsection{Future Work}

Through this research project, a number of new systems were developed, and models validated, but additional work can be done in this area, through further refinement of the component models, and/or through the applications within a complete system. When looking at the model development for the components, the ice storage model had a number of underlying assumptions that could not be validated using only the small scale, single pipe experimental test set-up. This includes whether a larger tank with cooling draws being taken will fully mix and reach approximately $0^{\circ} \mathrm{C}$ before ice starts forming, and that there is no interaction between the cooling pipes installed within the system (i.e. all pipes have equal ice accumulation). As the small-scale system has exhausted its potential, a full-scale tank should be constructed and fully instrumented. Using this tank model, many of the outstanding assumptions and questions could be measured and validated. This would also 
allow a CFD model of the tank to be validated, which could then be used to determine the impact of scaling the system to better match the building loads.

In addition to the confirming some of the assumptions, only one pipe configuration was examined within the study (straight, round pipe), alternate geometries could be explored within this large-scale set-up. This could include determining the potential benefit of using coiled pipes to increase the heat transfer and ice growth area, and therefore reducing the total number of pipes required. Alternatively, straight pipes could still be used, but fins added to the pipes, again increasing the heat transfer and ice growth area. Using fins with ice between them, could cause an issue where the fins would be bent or break with the expansion of ice. As such, not only would the heat transfer components need to be examined with this configuration, but the durability and resiliency of the fins for long term, daily use would as well.

Finally, through the ice storage validation, a number of the measurement uncertainties compounded, creating a significant experimental uncertainty. In building a full-scale test set-up, it would be important to design and budget for instrumentation with the accuracy required to reduce the experimental uncertainty on the cumulative measurements. Most notably, this would require the use of tightly calibrated thermopiles on each pipe or the use of secondary reference RTDs, flow meters accurate at very low flows, and the ability to accurately and preferably continuously measure that amount of ice formed in the system.

In terms of the total system, the results from this study show that a significant amount of cooling potential can be stored using the ice storage system, and when using the medium temperature chiller, can be achieved with decent efficiency and in a small volume. 
Based on the findings throughout, the shifting from peak to off-peak consumption is probably not the most optimal application for this system, as the increase in off-peak consumption is hard to overcome through peak savings. This system would be best used in a situation where excess power must be consumed, or it would be lost, or a penalty applied. Although this situation is rare, there are a few applications where this could be applicable.

Currently in Ontario, renewable contracts allow any excess produced electricity to be sold directly to the grid at the current rate or with the feed in tariff, at a premium. Many jurisdictions are going away from this model and are requiring the generated power to be used onsite, within some time period, be it 15 minutes to a couple of hours. At the end of the net-metering period, any surplus power is paid for by the utility provider at a rate much lower than what it would be purchased for. As such, storage systems, be it electrical batteries or thermal storage for either space heating or cooling, can be used to bring that net import/export to the grid to 0 , and therefore reduce the total cost, if the heating and cooling is required during a period where no generation is possible (typically overnight). As such, a modelling study should be conducted to determine the potential for reducing annual utility costs through this type of rate plan, and directly coupling the heat pump to the production of a house's PV system.

The second scenario where this could occur is on the grid level. With the slow ramp rates of many generating methods and the unpredictability of both renewable generation and overall consumer demand, there are periods in which there is an excess amount of electricity available on the grid. During these periods, the utility provider must dump the power, typically paying other utility providers to take it, causing negative market rates. During this study, the use of market rates was studied to determine the economic feasibility, 
but the control strategy remained time- and required cooling-based, with no feedback on the current market price. If the infrastructure was available to get live market pricing, control strategies could be developed that tie the amount of cooling stored to the live market price. This would allow the system to take advantage of low and negative market pricing periods, no matter what time of the day or how much cooling is required and allow that cheap or free cooling to be used when the market rate is much higher.

The final future project to build off this work is the introduction of new and emerging heat pump systems and refrigerants, that perform better at low temperatures and with large temperature lifts from the evaporator to the condenser. Most notably, of these new systems is the use of $\mathrm{CO}_{2}$ as a refrigerant. Because of the very high pressures the systems operate at, they are optimally designed to operate with huge changes in temperature. The downside to the systems is the very high initial capital costs, due to the limited availability and specialized equipment required in the cycles, that can handle and operate under the required pressures. As such, both technical and economic analyses must be conducted to determine whether $\mathrm{CO}_{2}$ systems would improve the life cycle economics of the system, and whether the energy savings offset the initial capital costs. 


\section{References}

[1] Natrual Resources Canada, "National Energy Use Database," Ottawa, Canada, 2020.

[2] Statistics Canada, "Households and the Environment: Energy Use," Statistics Canada, Ottawa, Ontario, 2011.

[3] Independent Electricity Systems Operator, "Ontario Demand Forecast," Toronto, Ontario, 2018.

[4] Ontario Ministry of Energy, "Achieving Balance - Ontario's Long Term Energy Plan," Toronto, 2013.

[5] I. Urgursal and A. Farhat, "Greenhouse gas emission intensity factors for marginal electricity," International Journal of Energy Research, vol. 34, pp. 1309-1327, 2010.

[6] Ontario Energy Board, "Electrcity Proces," 02 November 2015. [Online]. Available:

http://www.ontarioenergyboard.ca/oeb/Consumers/Electricity/Electricity\%20Pric es. [Accessed March 2016].

[7] Nova Scotia Power, "Time-of-Day Rates," 1 January 2016. [Online]. Available: http://www.nspower.ca/en/home/for-my-home/heating-solutions/electricthermal-storage/tod-rates/default.aspx. [Accessed March 2016].

[8] Pacific Gas and Electric, "Time-of-Use Plans," [Online]. Available: http://www.pge.com/en/myhome/saveenergymoney/plans/tou/index.page. [Accessed March 2016].

[9] Independent Electricity System Operator, "Save on Energy," [Online]. Available: https://saveonenergy.ca/. [Accessed March 2016].

[10] G. Emmi, A. Zarrella and M. De Carli, "A heat pump coupled with photovoltaic thermal hybrid solar collectors: A case study of a multi-source energy system," Energy Conversion and Management, vol. 151, pp. 386-399, 2017.

[11] K. Chua, S. Chou and V. Yang, "Advances in het pump systems: A review," Applied Energy, vol. 87, pp. 3611-3624, 2010.

[12] J. Chu, "Evaluation of a Dual Tank Indirect Solar-Assisted Heat Pump System for a High Performance House," M.A.Sc. Thesis, Carleton University, Ottawa, On., 2014.

[13] M. Moran and H. Shapiro, Fundamentals of Engineering Thermodynamics Sixth Edition, John Wiley and Sons, Inc., 2008.

[14] P. Pinel, C. A. Cruickshank, I. Beausoliel-Morrison and A. Wills, "A Review of Available Methods for Seaonal Storage of Solar Thermal Energy in Residential Applications," Renewable and Sustainable Energy Reviews, no. 15, pp. 33413359, 2011.

[15] C. A. Cruickshank and C. Baldwin, "Sensible and Thermal Energy Storage: Diurnal and Seasonal," in Storing Energy: with Special Reference to Renewable Energy Sources, Elsevier, 2016, pp. 291-309.

[16] Ontario Energy Board, "Historical Electricty Prices," 15 October 2015. [Online]. Available: 
http://www.ontarioenergyboard.ca/OEB/Consumers/Electricity/Electricity+Price s/Historical+Electricity+Prices. [Accessed April 2016].

[17] D. Y. Goswami and F. Kreith, Energy Efficiency and Renewable Energy Handbook, Second Edition, Boca Raton, Florida: CRC Press, 2016.

[18] Independent Electrical Service Operator, "Peaksaver PLUS," 2016. [Online]. Available: https://www.saveonenergy.ca/Consumer/Programs/PeaksaverPlus.aspx. [Accessed April 2016].

[19] M. Goulden, B. Bedwell, S. Rennick-Egglestone, T. Rodden and A. Spence, "Smart grids, smart users? The role of the user in demand side," Energy Research \& Social Science, vol. 2, pp. 21-29, 2014.

[20] A. Pina, C. Silva and P. Ferrao, "The impact of demand side management strategies in the penetration of renewable electricity," Energy, vol. 41, pp. 128137, 2011.

[21] F. Fernandes, H. Morais, Z. Vale and C. Ramos, "Dynamic load management in a smart home to participate in demand response events," Energy and Buildings, vol. 82, pp. 592-606, 2014.

[22] T. Logenthiran, D. Srinivasan and T. Z. Shun, "Demand Side Management in Smart Grid Using," IEEE Transactions on Smart Grids, vol. 3, no. 3, pp. 12441252, 2012.

[23] L. Logenthiran, D. Sirinivasan and K. Vanessa, "Demand side management of smart grid: Load shifting and incentives," Journal of Renewable and Sustainable Energy, vol. 6, no. 3, 2014.

[24] M. A. Lopez, S. de la Torre, S. Martin and J. A. Aguado, "Demand-side management in smart grid operation considering electric vehicles load shifting and vehicle-to-grid support," International Journal of Electrical Power \& Energy Systems, vol. 64, pp. 689-698, 2015.

[25] E. Thomas, R. Sharma and Y. Nazarathy, "Towards demand side management control using household specific Markovian models," Automatica, vol. 101, pp. 450-457, 2019.

[26] B. Esther and K. Kumar, "A survey on residential demand side management architecture, approaches, optimization models and methods," Renewable and Sustainable Energy Reviews, vol. 59, pp. 342-351, 2016.

[27] Y. Zhang, G. Zhou, K. Lin, Q. Zhang and H. Di, "Application of latent heat thermal energy storage in buildings: State-of-the-art and outlook," Building and Environment, vol. 42, pp. 2197-2209, 2007.

[28] W. Qureshi, N. Nair and M. Farid, "Impact of energy storage in buildings on electricity demand side management," Energy Conversion and Management, vol. 52, no. 5, pp. 2110-2120, 2011.

[29] L. Paull, H. Li and L. Chang, "A novel domestic electric water heater model for a multi-objective deamnd side management program," Electric Power Systems Research, vol. 80, pp. 1446-1451, 2010.

[30] M. H. Nehrir, B. J. LaMeres and V. Gerez, "A customer-interactive electric water heater demand-side management strategy using fuzzy logic," Power Engineering 
Society 1999 Winter Meeting, IEEE., vol. 1, pp. 433-436, 1999.

[31] Z. Chen, L. Wu and Y. Fu, "Real-Time Price-Based Demand Response Management for Residential Appliances via Stochastic Optimization and Robust Optimization," IEEE Transactions on Smart Grid, vol. 3, no. 4, pp. 1822-1831, 2012.

[32] Gabarron Manufacturing Excellence, "ECOMBI," Off Peak Tariff, [Online]. Available: http://www.ecombi-northamerica.com/off-peak.html. [Accessed April 2016].

[33] Dimplex, "Quantum Storage Heaters," [Online]. Available: http://www.dimplex.com/en/renewable_energy/quantum_storage_heaters. [Accessed April 2016].

[34] A. Molina, A. Gabaldon, C. Alvarez, J. A. Fuentes and E. Gomez, "Electrical Thermal Storage Modeling: a Tool to Evaluate New opportunities and Bids for Residential Users in a Deregulated Market," in IEEE Bologna PowerTech Conference, Bologna, Italy, 2003.

[35] A. Arteconi, N. J. Hewitt and F. Polonara, "State of the art of thermal storage for demand-side management," Applied Energy, vol. 93, pp. 371-389, 2012.

[36] I. Sarbu and C. Sebarchievici, "General review of ground-source heat pump systems for heating and cooling of buildings," Energy and Buildings, vol. 70, pp. 441-454, 2014.

[37] C. Ellerbrok, "Potentials of demand side management using heat pumps with building mass as a thermal storage," Energy Procedia, vol. 46, pp. 214-219, 2013.

[38] K. Hedegaard and O. Balyk, "Energy system investment model incorporating heat pumps with thermal storage in buildings and buffer tanks," Energy, vol. 63, pp. 356-365, 2013.

[39] G. Masy, E. Georges, C. Verhelst, V. Lemort and P. Andre, "Smart grid energy flexible buildings through the use of heat pumps and building thermal mass as energy storage in the Belgian context," Science and Technology for the Built Environment, vol. 21, no. 6, pp. 800-811, 2015.

[40] R. Hirmiz, H. Teamah, M. Lightstone and J. Cotton, "Performance of heat pump integrated phase change material thermal storage for electric load shifting in building demand side management," Energy and Buildings, vol. 190, pp. 103$118,2019$.

[41] P. Moreno, A. Castell, C. Sole, G. Zsembinszki and L. Cabeza, "PCM thermal energy storage tanks in heat pump systems for space cooling," Energy and Buildings, vol. 82, pp. 399-405, 2014.

[42] A. Real, V. Garcia, L. Domenech, J. Renau, N. Montes and F. Sanchaz, "Improvement of a heat pump based HVAC system with PCM thermal storage for cold accumilation and heat dissipation," Energy and Buildings, vol. 83, pp. 108-116, 2014.

[43] A. Arteconi, N. J. Hewitt and F. Polonara, "Domestic demand-side management (DSM): Role of heat pumps and thermal energy storage (TES) systems," Applied 
Thermal Energy, vol. 51, pp. 155-165, 2013.

[44] I. Dincer and M. Rosen, Thermal Energy Storage; Systems and Applications, 2nd Edition, West Sussex, United Kingdom: John Wiley and Sons, Ltd., 2011.

[45] X. Wang, Y. Hwang and R. Radermacher, "Two-stage heat pump system with vapor-injected scroll compressor using R410A as a refrigerant," International Journal of Refrigeration, vol. 32, pp. 1442-1451, 2009.

[46] S. Bertsch and E. Groll, "Two-stage air source heat pump for residential heating and cooling applications in norther U.S. climates," International Journal of Refrigeration, vol. 31, pp. 1282-1292, 2008.

[47] J. Kim, J. Lee, H. Choi, S. Lee, S. Oh and W. Park, "Experimental Study of R134a/R410A cascade cycle for variable refrigerant flow heat pump systems," Journal of Mechanical Science and Technology, vol. 29, no. 12, pp. 5447-5458, 2015.

[48] C. A. Cruickshank, "Evaluation of a stratified multi-tank thermal storage for solar heating applications," Ph.D. Thesis, Queen's University, Kingston, Ontario, 2009.

[49] C. A. Cruickshank and S. J. Harrison, "Thermal Response of a Series-Connected Energy Storage to Multi-Day Charge Sequences," Journal of Solar Energy, no. 185, pp. 180-187, 2011.

[50] R. M. Dickinson and C. A. Cruickshank, "Exergy Analysis of a Multi-Tank Thermal Storage for Solar Heating Applications," International Journal of Exergy, vol. 15, no. 4, 2014.

[51] M. F. Lightstone, G. D. Raithby and K. G. T. Hollands, "Numerical Simulation of the Charging of Liquid Storage Tanks: Comparison with Experiment," ASME Journal of Solar Energy Engineering, vol. 111, pp. 225-231, 1989.

[52] J. E. B. Nelson, A. R. Balakrishnan and A. S. Murthy, "Experiments on stratified chilled-water tanks," International Journal of Refrigeration, vol. 22, pp. 216-234, 1999.

[53] M. A. Karim, "Experimental investigation of a stratified chilled-water thermal stroage system," Journal of Applied Thermal Engineering, vol. 31, pp. 18531860, 2011.

[54] Z. Z., W. D. Turner, Q. Chen, C. Xu and S. Deng, "Tank size and operating strategy optimization of a stratified chilled water system," Applied Thermal Engineering, vol. 31, pp. 2656-2664, 2011.

[55] M. J. Sebzali, B. Ameer and H. J. Hussain, "Comparison of energy performance and economics of chiller water thrmal storage and conventional air-conditioning systems," Energy and Buildings, vol. 69, pp. 237-250, 2014.

[56] D. Nizami, M. Lightstone, S. Harrison and C. A. Cruickshank, "Simulation of the interaction of a solar domestic hot water tank system with a compact plate heat excahnger," in Proceedings of the Joint Conference of the Canadian Solar Buildings Research Network and Solar Energy Society of Canada Inc., Fredericton, New Brunswick, 2008. 
[57] R. Consul, I. Rodriguez, C. Perez-Segarra and M. Soria, "Virtual prototyping of storage tanks by means of three-dimensional CFD and heat transfer numerical simulations," Solar Energy, vol. 77, no. 2, pp. 179-191, 2004.

[58] L. Shah and S. Furbo, "Entrance effects in solar storage tanks," Solar Energy, vol. 75, no. 4, pp. 337-348, 2003.

[59] W. Yaici, M. Ghorab, E. Entchev and S. Hayden, "Three-dimensional unsteady CFD simulations of a thermal storage tank performance for optimum design," Applied Thermal Engineering, vol. 60, no. 2, pp. 152-163, 2013.

[60] C. A. Cruickshank and S. Harrison, "Simulation and testing of stratified multitank, thermal storages for solar heating systems," in Proceedings of the EuroSun 2006 Conference, Glasgow, Scotland, 2006.

[61] B. Newton, "Modeling of solar storage tanks," M.Sc. Thesis, University of Wisconsin-Madison, 1995.

[62] "TRNSYS: A Transient Simulation Program," University of Wisconsin Solar Energy Laboratory, Madison, Wisconsin, 2015.

[63] Y. Yau and B. Rismanchi, "A review on cool thermal storage technologies and operating strategies," Renewable and Sustainable Energy Reviews, vol. 16, pp. 787-797, 2012.

[64] Y. Sun, S. Wang, F. Xiao and D. Gao, "Peak load shifting control using different cold thermal energy storage facilities in commercial buildings: A review," Energy Conversion and Management, vol. 71, pp. 101-114, 2013.

[65] D. Erdemir and N. Altuntop, "Investigation of the effect of using graded porosity on the thermal performance of the encapuslated ice thermal energy storage tank," Energy Storage, vol. 1, no. 1, 2019.

[66] A. Lee and J. Jones, "Laboratory performance of an ice-on-coil thermal-energy storage system for residnetial and light commercial applications," Energy, vol. 21, no. 2, pp. 115-130, 1996.

[67] H. Sait, "Experimental study of water solidification phenomenon for ice-on-coil therma energy storage application utilizing falling film," Applied Thermal Engineering, vol. 146, pp. 135-145, 2019.

[68] The University of Wisconsin - Madison, "207 Ice Storage Tank," [Online]. Available: http://sel.me.wisc.edu/trnsys/trnlib/trnlib16/thermal\%20storage/type207.txt. [Accessed 2016].

[69] T. Jekel, J. Mitchell and S. Klein, "Modeling of Ice-storage tanks," in ASHRAE, Atlanta, Georgia, 1993.

[70] K. Drees and J. Braun, "Modeling of area-constrained ice storage tanks," $H V A C \& R$ Research, vol. 1, no. 2, 1995.

[71] B. Soltan and M. Ardehali, "Numerical simulationof water solidification phenomenon for ice-on-coil thermal energy storage applications," Energy Conversion and Management, vol. 44, pp. 85-92, 2003.

[72] T. Jekel, "Modeling of Ice-Storage Systems," M.S. Thesis University of Wisconsin - Madinson, Madison, Wisconsin, 1991. 
[73] C. Baldwin, "Design and Construction of an Experimental Apparatus to Assess the Performance of a Solar Absorption Chiller with Integrated Thermal Storage," M.A.Sc. Thesis - Carleton University, Ottawa, Ontario, 2013.

[74] S. Brideau, I. Beausoliel-Morrison and K. M. , "Empirical Model of a $11 \mathrm{~kW}$ (Nominal Cooling) R134a Water-Water Heat Pump," in eSim 2016, Hamilton, Ontario, 2016.

[75] DOW Chemical Company, "Guide to Glycols," DOW Chemical Companies, Midland, Michigan.

[76] G. Csordas, A. Brunger, K. Hollands and M. Lightstone, "Plume entrainment effects in solar domestic hot water systems employing variable-flow-rate control strategies," Solar Energy, vol. 49, no. 6, pp. 497-505, 1992.

[77] T. Bergman, A. Lavine, F. Incropera and D. Dewitt, Fundamentals of Heat and Mass Trasnfer: Seventh Edition, Hoboken, New Jersey: John Wiley \& Sons Inc., 2011.

[78] J. Holman, Heat Transfer: Tenth Edition, New York, New York: McGraw-Hill, 2010.

[79] Ministry of Municipal Affairs and Housing, "2012 Building Code Compendium," Toronto, Ontario, 2019.

[80] National Research Council, "National Energy Code of Canada for Buildings 2015," Ottawa, Ontario, 2015.

[81] Hydro Ottawa, "Residential Rates," [Online]. Available: https://hydroottawa.com/accounts-services/accounts/rates-conditions/residentialrates.

[82] Canadian Standards Association, "CAN/CSA-F379.1-88 Solar Domestic Hot Water Systems (Liquid to Liquid Heat Transfer)," CSA, Mississauga, On, 2004.

[83] B. Rose and E. Floehr, "Analysis of High Temperature Forecast Accuracy of Consumer Weather Forecasts from 2005-2016," Forecast Watch, Columbus, Ohio, 2017.

[84] C. Baldwin and C. Cruickshank, "Using forecased daily maximum temperatures to control a chiller thermal storage system," in ASME 2018 International Mechanical Engineering Congress and Exposition, Pittsburgh, PA., 2018.

[85] M. Mcclearn and K. Baum, "Ontario governement supports OPG proposal to operate Pickering nuclear station past planned 2024 closing," The Globe and Mail, 16 January 2020.

[86] National Energy Board, "Canada's Renewable Power Landscape - Energy Market Analysis 2017," National Energy Board, Calgary, Alberta, 2017.

[87] Indepenent Electrical System Operator, "HOEP Monthly Averages Report," 2020. [Online]. Available: http://reports.ieso.ca/public/PriceHOEPAverage/.

[88] T. Spears, "Ottawa misses a heat record, but sets humidex record," Ottawa Citizen, 3 July 2018.

[89] Natural Resources Canada, "Ground-Source Heat Pumps (Earth-Energy Systems)," Natural Resources Canada, Ottawa, Ontario, 2017. 
[90] Government of British Columbia - Building and Safety Standards Branch, "Determining ASHRAE 90.1-2010 Climate Zones," Governmnet of British Columbia, Victoria, British Columbia, 2014.

[91] Florida Power and Light, "Residential rates, clauses and storm factors," March 2018. [Online]. Available: https://www.fpl.com/rates/pdf/Mar2018Residential.pdf.

[92] Salt River Project, "SRP Time-of-Use Price PLan," March 2018. [Online]. Available: https://www.srpnet.com/prices/home/tou.aspx.

[93] Los Angeles Department of Water and Power, "Electric Rates," March 2018. [Online]. Available: https://www.ladwp.com/ladwp/faces/ladwp/residential/rcustomerservices/r-cs-understandingyourrates/r-cs-ur-electricrates.

[94] Portland General Electric, "Time of Use Pricing," March 2018. [Online]. Available: https://www.portlandgeneral.com/residential/power-choices/time-ofuse/time-of-use-pricing.

[95] National Grid - Massachusetts Electric, "Service Rates," March 2018. [Online]. Available: https://www.nationalgridus.com/MA-Home/Rates/Service-Rates.

[96] Toronto Hydro Corporation, "Residential Electricty Rates," March 2018. [Online]. Available:

http://www.torontohydro.com/sites/electricsystem/residential/rates/pages/resirate s.aspx.

[97] Greater Sudbury Utilities, "Rates Schedule," March 2018. [Online]. Available: http:/gsuinc.ca/shared_pages/rates-schedule/.

[98] U.S. Department of Energy, "Weather Data," NREL, [Online]. Available: https://energyplus.net/weather.

[99] Bank of Canada, "Inflation," Bank of Canada, [Online]. Available: https://www.bankofcanada.ca/core-functions/monetary-policy/inflation/. [Accessed 2020].

[100] Ontario Energy Board, "Historical electricity rates," Queen's Printer for Ontario, 2019. [Online]. Available: https://www.oeb.ca/rates-and-your-bill/electricityrates/historical-electricity-rates. [Accessed 2020].

[101] A. Fremeth, "A Historical and Comparative Perspective on Ontario's Electricty Rates," Energy Regulatation Quarterly, Ottawa, Canada, 2018.

[102] "Central Air Conditioner Prices in Canada," 2019. [Online]. Available: https://www.furnaceprices.ca/air-conditioners/central-air-conditioner-pricescanada/. [Accessed January 2020].

[103] Natural Resources Canada, "Energy Markets Fact Book 2014-2015," Natural Resources Canada, Ottawa, Ontario, 2014.

[104] The World Bank, "Electrical Power Consumption (kWh per Capita)," The World Bank Group, Washington, DC., 2014.

[105] Canadian Electrcitiy Association, "Key Canadian Electricity Statistics," Canadian Electricty Association, Ottawa, Ontario, 2013.

[106] Statistics Canada, "Table 127-0002 - Electric power generation, by class of electricity producer, monthly," Statistics Canada, Ottawa, Ontario, 2016. 
[107] Independent Electricity Service Operator, "Ontario Energy Output and Capacity," 2016. [Online]. Available: http://www.ieso.ca/Pages/Ontario's-PowerSystem/Supply-Mix/default.aspx.

[108] Independent Electricity Service Operator, "Generator Output by Fuel Type Hourly Report," 01 January 2016. [Online]. Available: http://reports.ieso.ca/public/GenOutputbyFuelHourly/.

[109] Independent Electricity Service Operator, "Data Directory - Hourly Ontario and Market Demands - 2002-Present," 01 2016. [Online]. Available: http://ieso.ca/Pages/Power-Data/Data-Directory.aspx.

[110] CTV Ottawa, "February 2015 Coldest in 115 Years," 27 February 2014. [Online]. Available: http://ottawa.ctvnews.ca/february-2015-coldest-in-115years-1.2257188.

[111] Environment Canada, "Canada's Emissions Trends," Environment Canada, Ottawa, ON, 2013.

[112] O. Edenhofer, R. Pichs-Madruga, Y. Sokona, K. Seyboth, P. Matschoss, S. Kadner, T. Zwickel, P. Eickemeier, G. Hansen, S. Schlömer and C. v. Stechow, "IPCC Special Report on Renewable Energy Sources and Climate Change Mitigation," Cambridge University Press, Cambridge, United Kingdom, 2011.

[113] W. Moomaw, G. Burgherr, M. Heath, J. Lenzen, A. Nyboer and A. Verbruggen, "Annex II: Methodology, IPCC SPecial Report on Renewable Energy Sources and Climate Change Mitigation," Cambridge University Press, Cambridge, United Kingdom, 2011. 


\section{Appendices}

\section{Appendix A Energy Landscape in Canada and Ontario}

\section{A.1 Energy Landscape in Canada}

Canada is a significant player in the global electrical market, with electrical generation providing the backbone to the Canadian economy. Canada is responsible for $3 \%$ of worldwide electricity generation, ranking $6^{\text {th }}$ in the world behind China, United States, India, Russia and Japan [103]. When factoring in population, Canada also ranks $6^{\text {th }}$ in electrical generation per capita, at $15614 \mathrm{kWh}$ in 2012 [104]. Additionally, Canada is the $2^{\text {nd }}$ largest exporter of electricity behind Germany, and accounting for $9 \%$ of the 677 TWh exported worldwide in 2012 [103]. All of Canada's electricity exports are to the United States, where 58 TWh were exported in 2012, contributing almost $\$ 2$ billion to the Canadian economy [105].

Electricity in Canada is predominantly governed at the provincial level, with each

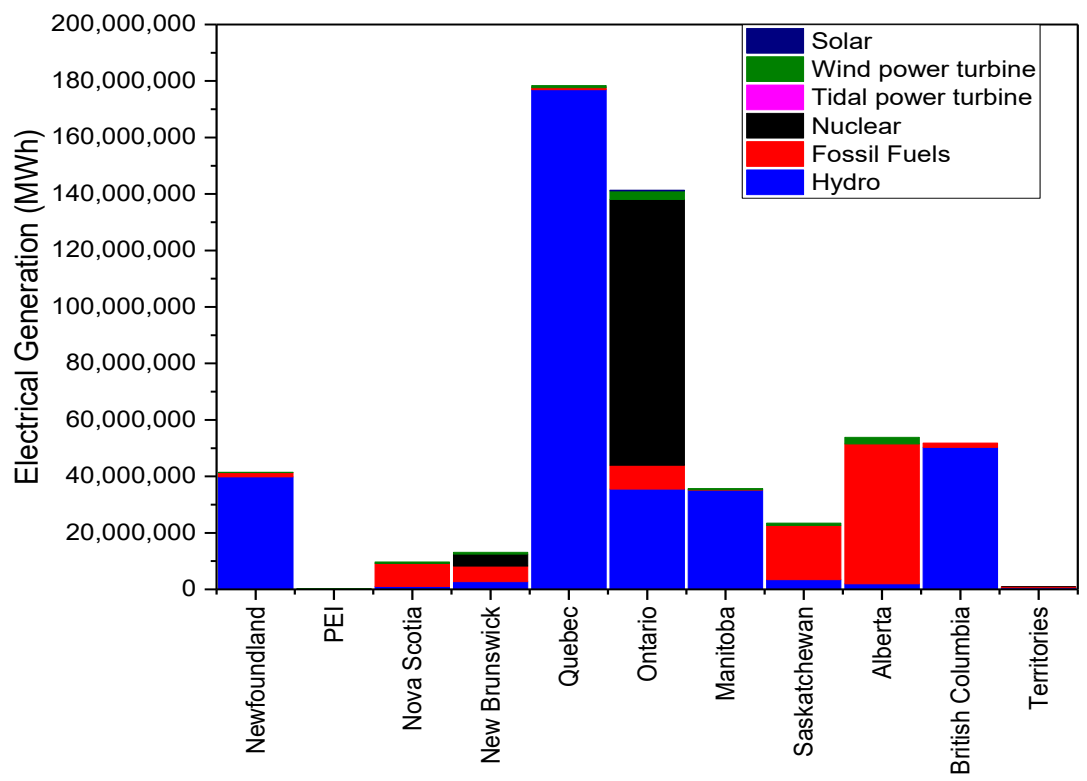

Figure A-1: Electrical generating source by Canadian province 
province forming a unique electricity jurisdiction, within individual regulation, generation methods, billing practices and distribution systems. These grids are interconnected with many grids in Northern United States, allowing for the exportation and importation of electricity, between provinces and also between provinces and the United States. As a result of these independent electrical jurisdictions, each has its own breakdown of electrical generation source, which includes fossil fuels, hydro, nuclear, and renewable energy sources. Consequently, each province's power supply has a different greenhouse gas intensity. Figure A-1 shows the generation by source for each province for 2015 [106].

When examining Canada's electrical generation as a whole by month and by generation source as shown in Figure A-2, it can be seen that Canada experiences a peak load during the winter months, as a result of Canada's heating dominated climate [106]. A second, smaller local peak is also present during July and August, when an increased electrical load is present for space cooling. A similar analysis can be conducted for each province in Canada, examining periods of peak loading and the composition of the power supply by generation source. A primary focus of this work is to reduce the peak loads

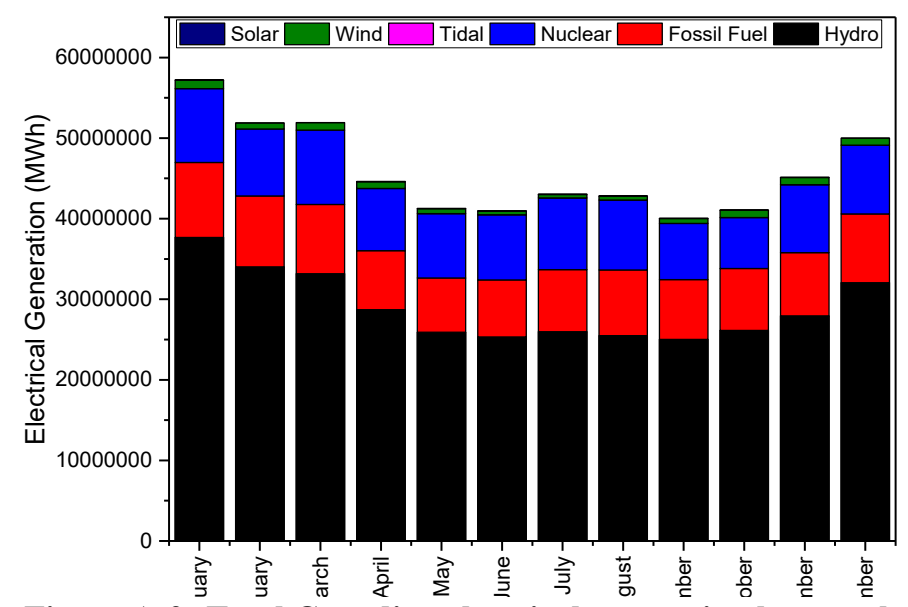

Figure A-2: Total Canadian electrical generation by month 
placed on the electrical grid. As such, analyzing the jurisdiction of interest in detail, the periods of peak loads, and the effect the peak load has on the electrical grid, the composition of the generation source and the change in greenhouse gas emissions can all be determined. As this thesis focuses on systems optimized for Ontario, the following section will present in detail the composition of the Ontario electrical grid and the influence peak loading has on the grid.

\section{A.2 Ontario Electrical Grid}

Ontario has the most diverse electrical grid in Canada, with four separate sources accounting for more than $5 \%$ of electrical generation in 2015 . These included wind (5.6\%), natural gas $(10.3 \%)$, nuclear $(60.3 \%)$ and hydro $(23.4 \%)$. The ratio of generation an any given point is dependent on consumer demand and production from variable, less controllable, sources including wind and solar [107]. In Ontario, the base electrical load is primarily met using hydro and nuclear electrical generation, while natural gas plants are turned on and off and modulated to meet the changing demand on an hour by hour basis, causing large fluctuations in the amount of electricity generated using natural gas power plants. This base load and peak loads can be seen in Figure A-3, which shows an hour by hour comparison of generating sources in Ontario for 2015 [108]. From this figure, it can also be observed that in 2015, peak electrical consumption occurred during the winter months (January and February), with a second smaller peak occurring later summer (August). As January and February 2015 were some of the coldest months on record in Ontario, historical consumption for 2002 to 2015 was obtained, and the average 
consumption for each hour of the year was plotted to determine the historical electrical peaks in Ontario, which can be seen in Figure A-4 [109, 110].

From Figure A-4, it can clearly be seen that historically in Ontario, there are two peaks in electrical consumption, with the first (and slightly larger) occurring early in the year when heating loads are greatest, and the second occurring in the middle of the summer when cooling loads are at their greatest. Of equal importance is the significant decrease in electrical consumption during the shoulder seasons as a result of almost no heating or

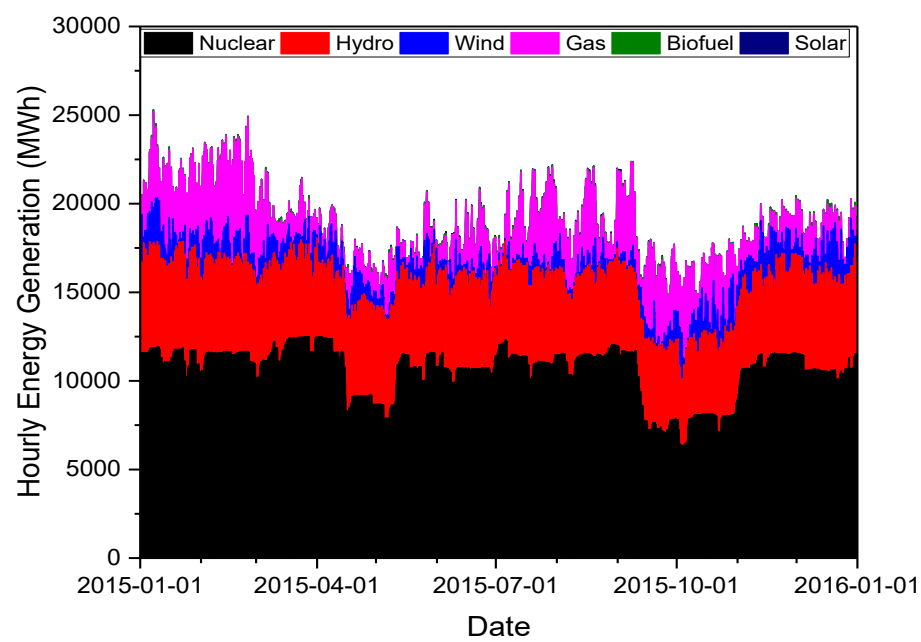

Figure A-3: Hourly electrical generation by source in Ontario for 2015

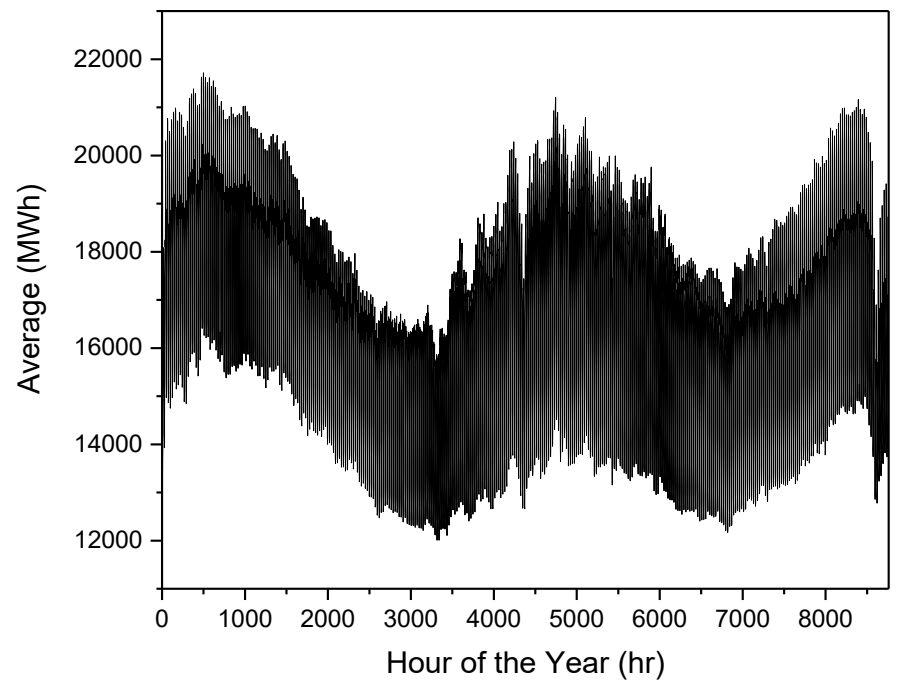

Figure A-4: Average historical electrical consumption by hour in Ontario (2002-2015) 
cooling load. From this data, it is clear that any work to reduce the peak load placed on the electrical grid in Ontario must focus on both the heating cooling seasons as opposed to focusing solely on one season.

\section{A.3 Influence of Peak Electrical Loads on Greenhouse Gas Emissions}

As shown in Figure A-3, the composition of electrical generation source varies throughout the year, and is in part, dependent on the demand the grid is experiencing. As demand increases, typically an increase in generation from natural gas power plants is observed, while when demand is lower, nuclear and hydro generation can meet most, if not all of the demand. As a result, the mix of generation sources varies hour to hour in Ontario, and consequently the greenhouse gas intensity (greenhouse gas emissions per unit of electricity generated) varies on an hourly basis. Before the greenhouse gas intensity can be calculated on an hourly basis, the emissions for each generating source must be determined.

\section{A.4 Calculating Greenhouse Gas Emissions from Electrical Generation}

To calculate the greenhouse gas emissions as a result of electrical, the quantity of greenhouse gases emitted per kilowatt-hour of electricity must be utilized. A study was conducted by Farhat and Ugursal [5] that examined the greenhouse gas emissions as a result of the marginal (electricity generation to meet the peak electrical load) for each province in Canada, and in more detail for Ontario, where marginal values were determined on a monthly basis. The marginal values were determined, as it was justified that any electricity savings would first remove generation from the peak generation capacity and not the base production. Although a valid assumption, this study does not provide the actual greenhouse gas emissions for each kilowatt-hour of generation in Ontario, and the data 
used is only up to 2008. Since 2008, Ontario has phased out its coal power plants and significantly increased renewable generation capacity, and as a result has significantly reduced its greenhouse gas emissions as a result of electrical generation [111]. Additionally, this report only reports marginal factors on a monthly basis, whereas hourly data is required for the proposed thesis. As such, new figures for greenhouse gas emissions for recent years had to be calculated.

The Independent Electricity System Operator (IESO) in Ontario publishes extensive electricity data on an hourly basis for generation and demand in Ontario. Included in total electrical generation by source on an hourly basis (Data for 2015 is shown in Figure A-3). To use the IESO generation data to calculate hourly greenhouse gas emissions, the emissions released per kilowatt-hour of electrical generation for each different source of electrical data must be determined. There is extensive data with thousands of scholarly articles pertaining to greenhouse gas emissions, with a large variation in reported values observed. This same problem was addressed by the United Nations Intergovernmental Panel on Climate Change in 2011 as part of the Special Report on Renewable Energy Sources and Climate Change [112]. The Panel reviewed more than 2100 sources, narrowing it down to 296 sources that provided reliable estimates of greenhouse gas emissions by source [113]. The Panel also settled on a lifecycle approach, where the emissions required to construct and decommission the power generation plant are considered in conjunction with greenhouse gas emissions of the fuel source. From this 
data, the minimum value, maximum value, and the $25^{\text {th }}, 50^{\text {th }}$ and $75^{\text {th }}$ percentile was reported and is indicated in Table A-1 below for pertinent technologies in Ontario.

Table A-1: Greenhouse gas intensity by generating source in $\mathrm{g} \cdot \mathrm{CO}_{2}$ equivalent $/ \mathrm{kWh}$

\begin{tabular}{c|ccccc}
\hline Generating Source & $\begin{array}{c}50^{\text {th }} \\
\text { Percentile }\end{array}$ & $\begin{array}{c}25^{\text {th }} \\
\text { Percentile }\end{array}$ & $\begin{array}{c}75^{\text {th }} \\
\text { Percentile }\end{array}$ & Minimum & Maximum \\
\hline Nuclear & 16 & 8 & 45 & 1 & 220 \\
Hydro & 4 & 3 & 7 & 0 & 43 \\
Natural Gas & 469 & 422 & 548 & 290 & 930 \\
Wind & 12 & 8 & 20 & 2 & 81 \\
Solar & 46 & 29 & 80 & 5 & 217 \\
Biomass & 18 & -360 & 37 & -633 & 75 \\
\hline
\end{tabular}

Based on the findings of the IPCC, the $50^{\text {th }}$ percentile values will be used for the remainder of this work, however comparison may be made with both the $25^{\text {th }}$ and $75^{\text {th }}$ percentiles. The minimum and maximum values will not be employed as these are outliers compared to the bulk of the data presented. 
Applying the values developed by the IPCC, the hourly greenhouse gas production in Ontario on an hourly basis can be calculated and is plotted in Figure A-5. From this graph, it is noted that the electricity produced from natural gas produces a significant portion of all greenhouse gases in Ontario, but is also highly variable throughout the year, with peaks coinciding with the peak electrical demand in Ontario. This data was further analyzed to look at how the month of the year, day of the week and time of the day influenced greenhouse gas emissions, with the monthly greenhouse gas intensity shown in Figure A-7, the average intensity for each day of the week shown in Figure A-8 and the average intensity for each hour of the day shown in Figure A-6. From this series of graphs, the greenhouse gas intensity of electrical generation is lowest in April, May, November and December, on weekends and overnight periods. Greenhouse gas intensity increases throughout the day, peaking in late afternoon/early evening, while intensity is much greater during the week compared to the weekend, but is relatively constant through the week. Using this data, a good picture of when energy consumption should be avoided and the

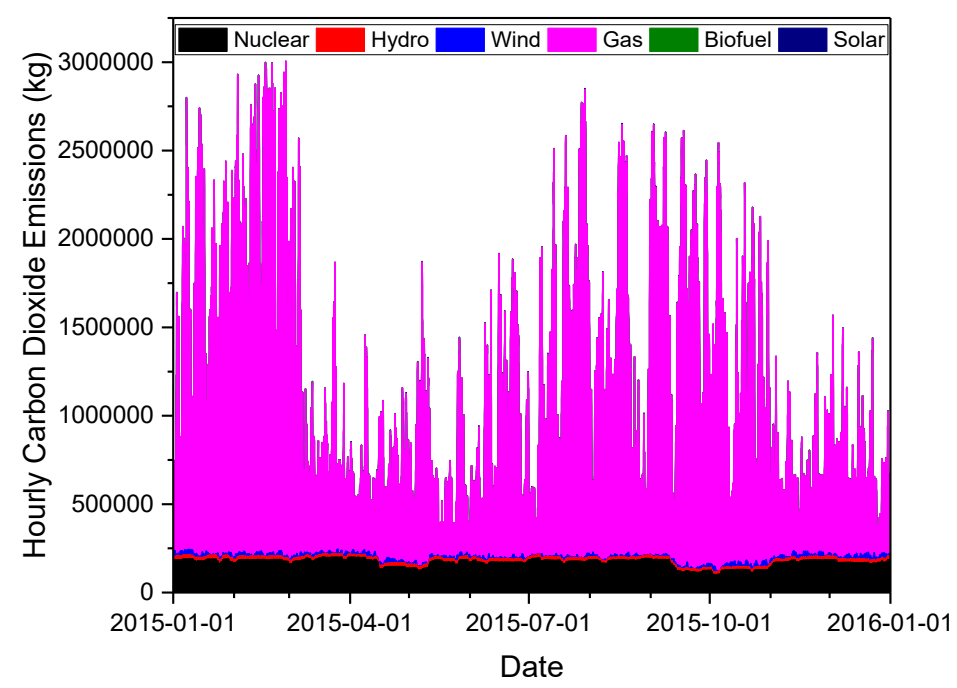

Figure A-5: Hourly greenhouse gas emissions in Ontario on an hourly basis and by generating source for 2015 
periods in which an effort should be made to shift consumption to.

\section{A.5 Time-of-Use Billing}

With the introduction of the smart meter to every electrical consumer, Ontario started transitioning to a time-of-use billing practice in May of 2006. Time-of-use billing charges consumers based on when they use electricity, with, in the case of Ontario, three pricing tiers for set periods during the year. This allows the utilities to charge a premium for electricity used during typical periods of greatest demand, while charging a must lower
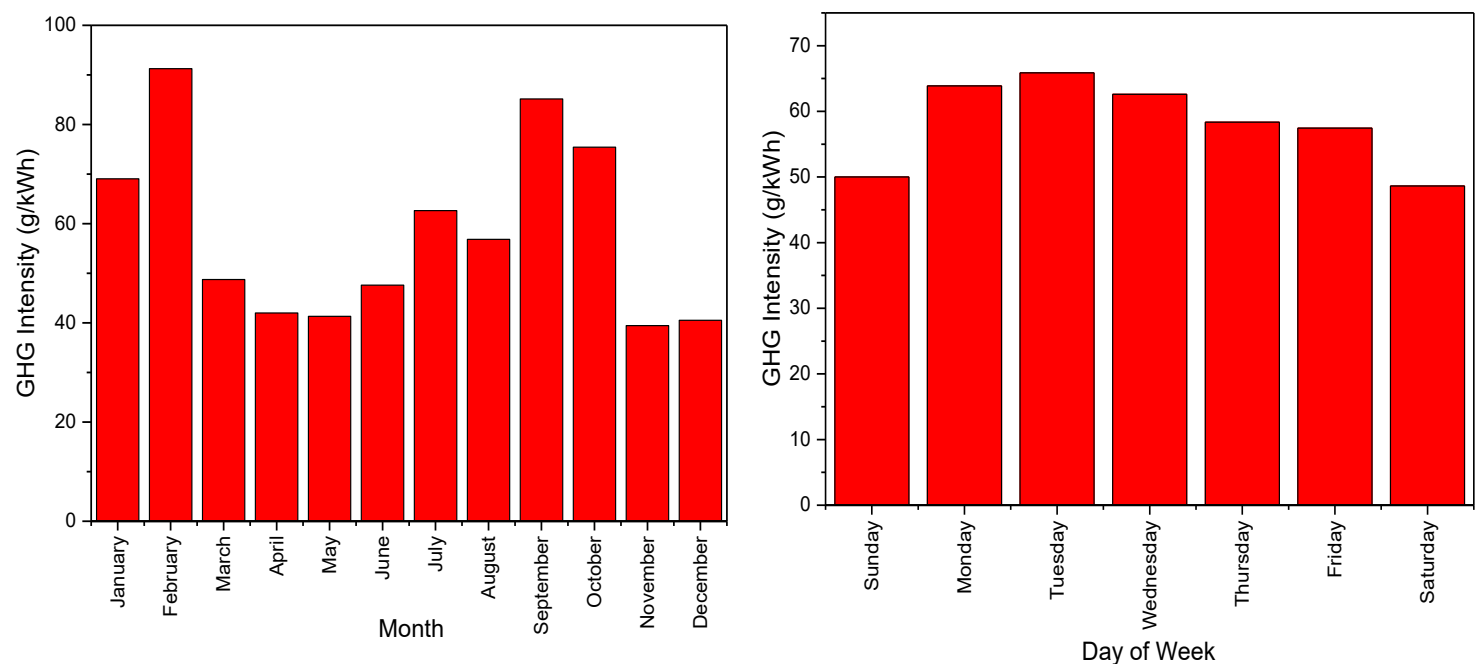

Figure A-7: Greenhouse gas intensity by month Ontario 2015

Figure A-8: Greenhouse gas intensity by day of week - Ontario 2015

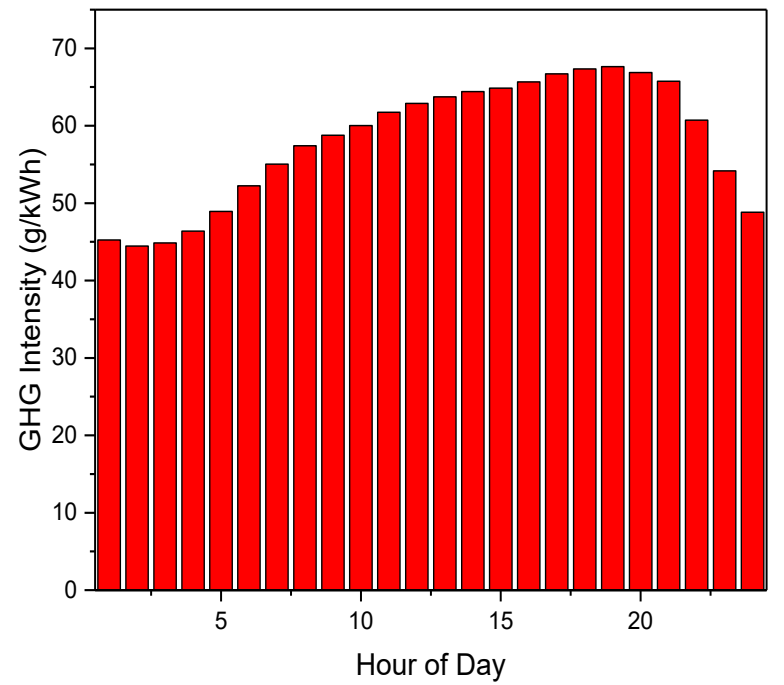

Figure A-6: Greenhouse gas intensity by hour of the day - Ontario 2015 
rate for electricity used during non-peak periods. Currently, in Ontario, electricity during peak periods cost 18 cents/kWh, while off-peak electricity cost 8.7 cents $/ \mathrm{kWh}$ (creating a $110 \%$ premium on electricity consumed during peak periods). For periods of moderate demand, a mid-peak rate of 13.2 cents/kWh [16]. As peak electrical demand varies from summer months (where peak demand occurs early in the morning and early evening) and summer months (during the afternoon period), a different rate schedule applies for each period. The rate schedule is given in Table A-2

Table A-2: Classification of usage periods in Ontario

\begin{tabular}{l|ccc|c}
\hline \multirow{2}{*}{ Season } & \multicolumn{3}{|c|}{ Weekdays } & $\begin{array}{c}\text { Weekends } \\
\text { and Holidays }\end{array}$ \\
\cline { 2 - 5 } & On-Peak & Mid-Peak & Off-Peak & Off-peak \\
\hline $\begin{array}{l}\text { Summer } \\
\text { (May-Oct) }\end{array}$ & $11 \mathrm{am}-5 \mathrm{pm}$ & $\begin{array}{c}7 \mathrm{am}-11 \mathrm{am} \\
5 \mathrm{pm}-7 \mathrm{pm}\end{array}$ & $7 \mathrm{pm}-7 \mathrm{am}$ & $24 \mathrm{hr}$ \\
\hline $\begin{array}{l}\text { Winter } \\
\text { (Nov-Apr) }\end{array}$ & $\begin{array}{c}7 \mathrm{am}-11 \mathrm{am} \\
5 \mathrm{pm}-7 \mathrm{pm}\end{array}$ & $11 \mathrm{am}-5 \mathrm{pm}$ & $7 \mathrm{pm}-7 \mathrm{am}$ & $24 \mathrm{hr}$ \\
\hline
\end{tabular}

As one of the justifications for the proposed system it to take advantage of the premium pricing placed on electrical consumption, for the remainder of this thesis work, on-peak and off-peak periods will be defined by the times provided in Table A-2, which are in line with what is dictate by the Ontario Energy Board [16].

\section{A.6 Relationship between Peak Electrical Consumption and GHG Emissions}

As the peak periods defined by the Ontario Energy Board are based on the time of day and season of the year in which energy consumption is the greatest, it should translate that these are also the periods when GHG emissions are greatest. To determine if this holds true, the 


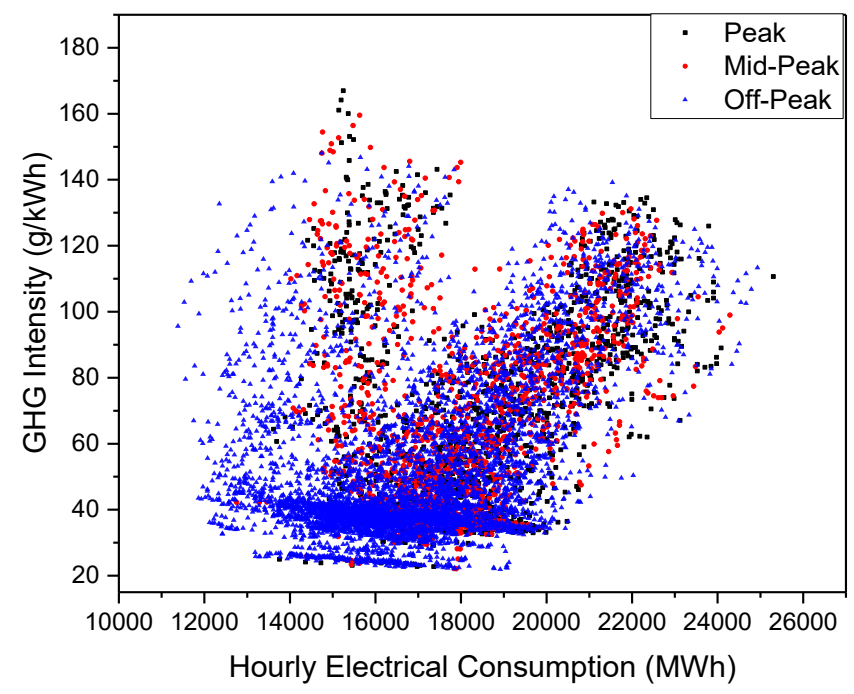

Figure A-9: Greenhouse gas intensity compared to total consumption and time-of-use

GHG intensity was plotted compared to the total generation for every hour, with the color of the point indicating whether it occurs during a peak, mid-peak or off-peak hour, as shown in Figure A-9. From this graph, it can be noted that peak and mid-peak hours tend to have a higher GHG intensity when compared to off-peak periods, where a large percentage of the time, the GHG intensity is low. The actual average GHG intensity for peak, mid-peak and off-peak periods was calculated for the complete year, as well as for summer and winter periods and is presented in Table A-3.

Table A-3: Greenhouse gas intensity by season and time-of-use

\begin{tabular}{c|ccc}
\hline \multirow{2}{*}{ Period } & \multicolumn{3}{|c}{ Greenhouse Gas Intensity $(\mathrm{g} / \mathrm{kWh})$} \\
& Off-Peak & Mid-Peak & Peak \\
\hline Annual & 53.4 & 65.2 & 68.1 \\
Summer & 54.9 & 70.5 & 76 \\
Winter & 51.8 & 59.9 & 60.2 \\
\hline
\end{tabular}




\section{Appendix B Heat Pump and Chiller Performance Map}

To model the heat pump in TRNSYS using Type 927, a performance map of the heat pump had to be developed and inserted into the model. To do this, the heat pump was run at steady state for 10 minutes with constant flow rates and temperatures entering the source and load side of the heat pump. The entering and exiting temperatures, flow rates and the amount of electrical energy consumed were measured and used to develop the performance chart. This was repeated for each combination of entering temperatures and flow rates. This was first done using water as the working fluid on both the source and load side, and then repeated using a 50/50 glycol/water solution by volume on the load side.

\section{B.1 Source Side Heat Transfer Fluid - Water}

The steady state tests were first conducted with water on the source side, and with entering source temperatures of $10^{\circ} \mathrm{C}$ to $25^{\circ} \mathrm{C}$ and load temperatures of $20^{\circ} \mathrm{C}$ to $40^{\circ} \mathrm{C}$. Table B-1 to B-3 show the heat transfer rates and electrical consumption under each test condition. Due to high pressures in the refrigerants and low temperature freeze conditions that were observed, some values had to be extrapolated, which was completed using a linear relationship, and these values are highlighted in green 
Table B-1: Electrical performance map for the heat pump (kW)

\begin{tabular}{|c|c|c|c|c|c|c|c|c|c|c|c|c|c|c|c|c|}
\hline \multirow{2}{*}{$\begin{array}{l}\begin{array}{l}\text { Flow Rate } \\
\text { (L/min) }\end{array} \\
\text { Source } \\
\text { Side }\end{array}$} & \multirow{2}{*}{$\begin{array}{l}\text { Load Side } \\
\text { Temperature } \\
\left({ }^{\circ} \mathrm{C}\right)\end{array}$} & \multicolumn{5}{|c|}{3} & \multicolumn{5}{|c|}{6} & \multicolumn{5}{|c|}{9} \\
\hline & & 20 & 25 & 30 & 35 & 40 & 20 & 25 & 30 & 35 & 40 & 20 & 25 & 30 & 35 & 40 \\
\hline \multirow{4}{*}{3} & 25 & 1.44 & 1.57 & 1.72 & 1.87 & 2.13 & 1.17 & 1.30 & 1.44 & 1.59 & 1.70 & 1.08 & 1.20 & 1.34 & 1.48 & 1.61 \\
\hline & 20 & 1.41 & 1.54 & 1.68 & 1.84 & 2.01 & 1.16 & 1.28 & 1.41 & 1.56 & 1.73 & 1.07 & 1.46 & 1.32 & 1.47 & 1.63 \\
\hline & 15 & 1.39 & 1.50 & 1.62 & 1.78 & 2.03 & 1.15 & 1.27 & 1.41 & 1.55 & 1.66 & 1.06 & 1.19 & 1.31 & 1.46 & 1.55 \\
\hline & 10 & 1.36 & 1.42 & 1.57 & 1.73 & 1.97 & 1.14 & 1.25 & 1.40 & 1.54 & 1.65 & 1.06 & 1.19 & 1.30 & 1.44 & 1.53 \\
\hline \multirow{4}{*}{6} & 25 & 1.46 & 1.60 & 1.78 & 1.93 & 2.09 & 1.18 & 1.31 & 1.41 & 1.60 & 1.71 & 1.06 & 1.20 & 1.35 & 1.49 & 1.67 \\
\hline & 20 & 1.40 & 1.61 & 1.74 & 1.92 & 2.08 & 1.18 & 1.30 & 1.42 & 1.59 & 1.72 & 1.06 & 1.20 & 1.34 & 1.49 & 1.63 \\
\hline & 15 & 1.43 & 1.56 & 1.69 & 1.86 & 1.99 & 1.16 & 1.29 & 1.43 & 1.56 & 1.70 & 1.08 & 1.19 & 1.33 & 1.47 & 1.60 \\
\hline & 10 & 1.39 & 1.50 & 1.64 & 1.81 & 1.95 & 1.14 & 1.27 & 1.39 & 1.55 & 1.71 & 1.06 & 1.18 & 1.31 & 1.45 & 1.57 \\
\hline \multirow{4}{*}{9} & 25 & 1.51 & 1.66 & 1.82 & 1.97 & 2.12 & 1.15 & 1.31 & 1.45 & 1.60 & 1.78 & 1.09 & 1.20 & 1.35 & 1.49 & 1.66 \\
\hline & 20 & 1.51 & 1.62 & 1.78 & 1.94 & 2.07 & 1.18 & 1.31 & 1.44 & 1.60 & 1.76 & 1.08 & 1.16 & 1.34 & 1.49 & 1.62 \\
\hline & 15 & 1.47 & 1.60 & 1.72 & 1.88 & 2.00 & 1.15 & 1.29 & 1.43 & 1.57 & 1.71 & 1.07 & 1.19 & 1.33 & 1.46 & 1.59 \\
\hline & 10 & 1.42 & 1.54 & 1.68 & 1.83 & 1.96 & 1.15 & 1.27 & 1.40 & 1.56 & 1.69 & 1.07 & 1.19 & 1.31 & 1.46 & 1.58 \\
\hline
\end{tabular}


Table B-2: Load side heat transfer rate $(\mathrm{kW})$

\begin{tabular}{|c|c|c|c|c|c|c|c|c|c|c|c|c|c|c|c|c|}
\hline \multirow{2}{*}{$\begin{array}{l}\begin{array}{l}\text { Flow Rate } \\
\text { (L/min) }\end{array} \\
\text { Source } \\
\text { Side }\end{array}$} & \multirow{2}{*}{$\begin{array}{l}\text { Load Side } \\
\text { Temperature } \\
\left({ }^{\circ} \mathrm{C}\right)\end{array}$} & \multicolumn{5}{|c|}{3} & \multicolumn{5}{|c|}{6} & \multicolumn{5}{|c|}{9} \\
\hline & & 20 & 25 & 30 & 35 & 40 & 20 & 25 & 30 & 35 & 40 & 20 & 25 & 30 & 35 & 40 \\
\hline \multirow{4}{*}{3} & 25 & 5.14 & 5.00 & 4.93 & 4.80 & 4.87 & 5.30 & 5.27 & 5.20 & 5.09 & 4.17 & 5.23 & 5.23 & 5.17 & 5.07 & 4.24 \\
\hline & 20 & 4.88 & 4.76 & 4.63 & 4.58 & 4.48 & 4.93 & 4.82 & 4.88 & 4.86 & 4.80 & 4.86 & 4.80 & 4.89 & 4.83 & 4.81 \\
\hline & 15 & 4.62 & 4.30 & 4.18 & 4.17 & 4.21 & 4.56 & 4.37 & 4.58 & 4.30 & 3.63 & 4.48 & 4.42 & 4.26 & 4.17 & 3.45 \\
\hline & 10 & 4.36 & 3.95 & 3.81 & 3.85 & 3.89 & 4.19 & 3.92 & 4.28 & 3.91 & 3.35 & 4.11 & 4.01 & 3.80 & 3.72 & 3.05 \\
\hline \multirow{4}{*}{6} & 25 & 5.30 & 5.49 & 5.55 & 5.39 & 5.28 & 6.15 & 6.07 & 5.10 & 5.80 & 4.69 & 5.75 & 5.88 & 6.11 & 5.94 & 5.70 \\
\hline & 20 & 4.92 & 5.46 & 5.23 & 5.14 & 4.98 & 5.75 & 5.65 & 5.34 & 5.31 & 4.49 & 5.20 & 5.78 & 5.60 & 5.61 & 5.12 \\
\hline & 15 & 5.10 & 5.00 & 4.88 & 4.84 & 4.72 & 5.27 & 5.19 & 5.17 & 4.95 & 4.33 & 5.35 & 5.33 & 5.25 & 5.17 & 4.78 \\
\hline & 10 & 4.68 & 4.48 & 4.41 & 4.43 & 4.25 & 4.76 & 4.74 & 4.60 & 4.51 & 4.40 & 4.87 & 4.78 & 4.77 & 4.59 & 4.54 \\
\hline \multirow{4}{*}{9} & 25 & 6.10 & 5.97 & 5.78 & 5.65 & 5.49 & 5.88 & 6.34 & 6.20 & 5.68 & 5.77 & 6.62 & 6.34 & 6.46 & 6.16 & 5.98 \\
\hline & 20 & 5.92 & 5.45 & 5.54 & 5.15 & 4.96 & 6.10 & 5.96 & 5.88 & 5.61 & 5.42 & 6.20 & 5.69 & 5.95 & 5.85 & 5.67 \\
\hline & 15 & 5.49 & 5.36 & 5.08 & 4.99 & 4.78 & 5.60 & 5.46 & 5.43 & 4.98 & 4.60 & 5.66 & 5.47 & 5.47 & 5.12 & 5.02 \\
\hline & 10 & 5.06 & 4.83 & 4.72 & 4.66 & 4.49 & 5.12 & 4.98 & 4.87 & 4.69 & 4.57 & 5.13 & 4.96 & 5.04 & 4.88 & 4.83 \\
\hline
\end{tabular}


Table B-3: Source side heat transfer rate (kW)

\begin{tabular}{|c|c|c|c|c|c|c|c|c|c|c|c|c|c|c|c|c|}
\hline \multirow{2}{*}{$\begin{array}{l}\text { Flow Rate } \\
\text { (L/min) } \\
\text { Source } \\
\text { Side }\end{array}$} & \multirow{2}{*}{$\begin{array}{l}\text { Load Side } \\
\text { Temperature } \\
\left({ }^{\circ} \mathrm{C}\right)\end{array}$} & \multicolumn{5}{|c|}{3} & \multicolumn{5}{|c|}{6} & \multicolumn{5}{|c|}{9} \\
\hline & & 20 & 25 & 30 & 35 & 40 & 20 & 25 & 30 & 35 & 40 & 20 & 25 & 30 & 35 & 40 \\
\hline \multirow{4}{*}{3} & 25 & 4.06 & 3.87 & 3.72 & 3.52 & 3.32 & 4.24 & 4.12 & 3.96 & 3.80 & 3.73 & 4.32 & 4.18 & 4.03 & 3.85 & 3.78 \\
\hline & 20 & 3.56 & 3.53 & 3.36 & 3.24 & 3.02 & 3.75 & 3.61 & 3.57 & 3.41 & 3.23 & 3.72 & 3.45 & 3.59 & 3.48 & 3.29 \\
\hline & 15 & 3.07 & 3.05 & 2.91 & 2.76 & 2.61 & 3.27 & 3.09 & 2.97 & 2.87 & 2.85 & 3.12 & 3.19 & 2.96 & 2.84 & 2.85 \\
\hline & 10 & 2.57 & 2.63 & 2.51 & 2.38 & 2.25 & 2.79 & 2.58 & 2.48 & 2.40 & 2.41 & 2.52 & 2.70 & 2.42 & 2.33 & 2.39 \\
\hline \multirow{4}{*}{6} & 25 & 5.08 & 4.50 & 4.35 & 4.10 & 3.87 & 5.29 & 5.08 & 4.87 & 4.53 & 4.35 & 5.62 & 5.29 & 4.93 & 4.69 & 4.34 \\
\hline & 20 & 4.53 & 4.23 & 4.00 & 3.79 & 3.53 & 4.78 & 4.57 & 4.20 & 4.07 & 3.90 & 4.90 & 4.62 & 4.44 & 4.24 & 4.01 \\
\hline & 15 & 3.91 & 3.82 & 3.62 & 3.42 & 3.27 & 4.28 & 4.10 & 3.88 & 3.65 & 3.52 & 4.22 & 4.14 & 3.95 & 3.73 & 3.59 \\
\hline & 10 & 3.51 & 3.33 & 3.15 & 3.01 & 2.82 & 3.74 & 3.60 & 3.34 & 3.21 & 3.00 & 3.79 & 3.56 & 3.36 & 3.21 & 3.00 \\
\hline \multirow{4}{*}{9} & 25 & 5.03 & 4.81 & 4.54 & 4.33 & 4.09 & 5.28 & 5.37 & 5.03 & 4.81 & 4.47 & 5.78 & 5.11 & 5.31 & 4.97 & 4.66 \\
\hline & 20 & 4.66 & 4.35 & 4.23 & 3.81 & 3.59 & 5.14 & 4.89 & 4.62 & 4.32 & 4.05 & 5.22 & 4.80 & 4.73 & 4.49 & 4.24 \\
\hline & 15 & 4.19 & 3.96 & 3.71 & 3.50 & 3.27 & 4.55 & 4.33 & 4.12 & 3.89 & 3.75 & 4.64 & 4.46 & 4.20 & 3.88 & 3.67 \\
\hline & 10 & 3.66 & 3.49 & 3.35 & 3.12 & 2.96 & 3.97 & 3.79 & 3.55 & 3.32 & 3.11 & 4.03 & 3.83 & 3.64 & 3.44 & 3.25 \\
\hline
\end{tabular}




\section{B.2 Source Side Heat Transfer Fluid - Water/Glycol Solution}

Once the performance map when using water as the working fluid on the source side was completed, a second set of experiments were run, using a 50/50 glycol/water solution by volume on the source side. The same procedure was utilized, however, an entering source side temperature of $5^{\circ} \mathrm{C}$ was added, while the $40^{\circ} \mathrm{C}$ load side temperature was removed. The results are shown in Table B-4 to B-6. 
Table B-4: Electrical performance map for the heat pump (kW)

\begin{tabular}{|c|c|c|c|c|c|c|c|c|c|c|c|c|c|}
\hline \multirow{2}{*}{$\begin{array}{l}\text { Flow Rate } \\
\text { (L/min) } \\
\text { Source } \\
\text { Side }\end{array}$} & \multirow{2}{*}{$\begin{array}{l}\text { Load Side } \\
\text { Temperature } \\
\left({ }^{\circ} \mathrm{C}\right)\end{array}$} & \multicolumn{4}{|c|}{3} & \multicolumn{4}{|c|}{6} & \multicolumn{4}{|c|}{9} \\
\hline & & 20 & 25 & 30 & 35 & 20 & 25 & 30 & 35 & 20 & 25 & 30 & 35 \\
\hline \multirow{5}{*}{3} & 25 & 1.35 & 1.49 & 1.64 & 1.73 & 1.12 & 1.26 & 1.40 & 1.53 & 1.07 & 1.19 & 1.32 & 1.45 \\
\hline & 20 & 1.32 & 1.44 & 1.60 & 1.73 & 1.12 & 1.24 & 1.37 & 1.51 & 1.05 & 1.17 & 1.30 & 1.44 \\
\hline & 15 & 1.28 & 1.43 & 1.56 & 1.74 & 1.11 & 1.23 & 1.35 & 1.49 & 1.05 & 1.15 & 1.28 & 1.42 \\
\hline & 10 & 1.27 & 1.37 & 1.54 & 1.69 & 1.09 & 1.19 & 1.32 & 1.46 & 1.05 & 1.14 & 1.27 & 1.40 \\
\hline & 5 & 1.24 & 1.33 & 1.48 & 1.68 & 1.09 & 1.18 & 1.29 & 1.43 & 1.05 & 1.12 & 1.25 & 1.38 \\
\hline \multirow{5}{*}{6} & 25 & 1.46 & 1.59 & 1.74 & 1.86 & 1.17 & 1.29 & 1.43 & 1.59 & 1.08 & 1.20 & 1.33 & 1.48 \\
\hline & 20 & 1.43 & 1.54 & 1.70 & 1.83 & 1.16 & 1.27 & 1.41 & 1.56 & 1.06 & 1.19 & 1.32 & 1.47 \\
\hline & 15 & 1.36 & 1.51 & 1.64 & 1.80 & 1.14 & 1.26 & 1.40 & 1.55 & 1.08 & 1.20 & 1.32 & 1.47 \\
\hline & 10 & 1.32 & 1.46 & 1.59 & 1.76 & 1.12 & 1.24 & 1.37 & 1.51 & 1.07 & 1.17 & 1.30 & 1.43 \\
\hline & 5 & 1.28 & 1.38 & 1.51 & 1.69 & 1.11 & 1.22 & 1.34 & 1.48 & 1.06 & 1.17 & 1.29 & 1.42 \\
\hline \multirow{5}{*}{9} & 25 & 1.50 & 1.64 & 1.80 & 1.88 & 1.18 & 1.30 & 1.44 & 1.59 & 1.08 & 1.17 & 1.33 & 1.48 \\
\hline & 20 & 1.48 & 1.60 & 1.75 & 1.88 & 1.17 & 1.29 & 1.43 & 1.59 & 1.07 & 1.20 & 1.33 & 1.48 \\
\hline & 15 & 1.41 & 1.55 & 1.66 & 1.88 & 1.16 & 1.29 & 1.42 & 1.57 & 1.08 & 1.19 & 1.34 & 1.47 \\
\hline & 10 & 1.38 & 1.51 & 1.64 & 1.78 & 1.14 & 1.26 & 1.40 & 1.56 & 1.07 & 1.19 & 1.31 & 1.46 \\
\hline & 5 & 1.34 & 1.44 & 1.59 & 1.72 & 1.12 & 1.24 & 1.37 & 1.51 & 1.07 & 1.17 & 1.30 & 1.44 \\
\hline
\end{tabular}


Table B-5: Load side heat transfer rate $(\mathrm{kW})$

\begin{tabular}{|c|c|c|c|c|c|c|c|c|c|c|c|c|c|}
\hline \multirow{2}{*}{$\begin{array}{l}\begin{array}{l}\text { Flow Rate } \\
\text { (L/min) }\end{array} \\
\text { Source } \\
\text { Side }\end{array}$} & \multirow{2}{*}{$\begin{array}{l}\text { Load Side } \\
\text { Temperature } \\
\left({ }^{\circ} \mathrm{C}\right)\end{array}$} & \multicolumn{4}{|c|}{3} & \multicolumn{4}{|c|}{6} & \multicolumn{4}{|c|}{9} \\
\hline & & 20 & 25 & 30 & 35 & 20 & 25 & 30 & 35 & 20 & 25 & 30 & 35 \\
\hline \multirow{5}{*}{3} & 25 & 4.36 & 4.33 & 4.23 & 3.78 & 4.47 & 4.46 & 4.47 & 4.28 & 4.50 & 4.44 & 4.44 & 4.21 \\
\hline & 20 & 4.05 & 3.95 & 3.98 & 3.45 & 4.08 & 4.14 & 4.14 & 3.79 & 4.05 & 4.03 & 4.09 & 3.90 \\
\hline & 15 & 3.35 & 3.58 & 3.51 & 3.45 & 3.70 & 3.64 & 3.67 & 3.65 & 3.66 & 3.66 & 3.65 & 3.62 \\
\hline & 10 & 3.30 & 3.19 & 3.15 & 3.14 & 3.39 & 3.27 & 3.25 & 3.35 & 3.34 & 3.28 & 3.30 & 3.32 \\
\hline & 5 & 2.80 & 2.81 & 2.78 & 2.83 & 3.00 & 2.86 & 2.85 & 2.89 & 2.93 & 2.89 & 2.90 & 2.90 \\
\hline \multirow{5}{*}{6} & 25 & 5.38 & 5.29 & 4.92 & 4.82 & 5.64 & 5.58 & 5.57 & 5.27 & 5.70 & 5.62 & 5.48 & 5.36 \\
\hline & 20 & 5.12 & 4.76 & 4.85 & 4.44 & 5.15 & 4.93 & 4.98 & 4.92 & 4.98 & 5.09 & 5.05 & 4.90 \\
\hline & 15 & 5.48 & 4.37 & 4.41 & 4.18 & 4.56 & 4.64 & 4.51 & 4.56 & 4.67 & 4.59 & 4.42 & 4.52 \\
\hline & 10 & 3.86 & 3.76 & 3.85 & 3.79 & 4.05 & 4.03 & 4.09 & 4.03 & 4.11 & 4.10 & 4.14 & 3.93 \\
\hline & 5 & 3.46 & 3.30 & 3.53 & 3.36 & 3.63 & 3.58 & 3.57 & 3.63 & 3.50 & 3.46 & 3.56 & 3.64 \\
\hline \multirow{5}{*}{9} & 25 & 5.90 & 5.72 & 5.64 & 4.96 & 6.17 & 6.06 & 5.94 & 5.70 & 6.21 & 5.76 & 5.79 & 5.67 \\
\hline & 20 & 5.51 & 5.36 & 5.26 & 4.89 & 5.71 & 5.60 & 5.47 & 5.39 & 5.58 & 5.64 & 5.55 & 5.33 \\
\hline & 15 & 4.89 & 4.79 & 4.19 & 4.68 & 5.01 & 5.07 & 5.00 & 4.89 & 5.10 & 4.98 & 4.98 & 4.88 \\
\hline & 10 & 4.34 & 4.28 & 4.09 & 4.29 & 4.55 & 4.43 & 4.47 & 4.69 & 4.67 & 4.53 & 4.34 & 4.37 \\
\hline & 5 & 4.22 & 3.80 & 3.71 & 3.76 & 4.07 & 3.98 & 4.09 & 4.10 & 4.11 & 3.95 & 4.11 & 4.04 \\
\hline
\end{tabular}


Table B-6: Source side heat transfer rate $(\mathrm{kW})$

\begin{tabular}{|c|c|c|c|c|c|c|c|c|c|c|c|c|c|}
\hline \multirow{2}{*}{$\begin{array}{l}\begin{array}{l}\text { Flow Rate } \\
\text { (L/min) }\end{array} \\
\text { Source } \\
\text { Side }\end{array}$} & \multirow{2}{*}{$\begin{array}{l}\text { Load Side } \\
\text { Temperature } \\
\left({ }^{\circ} \mathrm{C}\right)\end{array}$} & \multicolumn{4}{|c|}{3} & \multicolumn{4}{|c|}{6} & \multicolumn{4}{|c|}{9} \\
\hline & & 20 & 25 & 30 & 35 & 20 & 25 & 30 & 35 & 20 & 25 & 30 & 35 \\
\hline \multirow{5}{*}{3} & 25 & 2.28 & 2.16 & 2.05 & 2.04 & 2.41 & 2.30 & 2.14 & 2.12 & 2.42 & 2.32 & 2.27 & 2.15 \\
\hline & 20 & 2.11 & 2.13 & 1.94 & 1.41 & 2.23 & 2.20 & 2.14 & 1.93 & 2.24 & 2.25 & 2.18 & 2.09 \\
\hline & 15 & 1.46 & 1.49 & 1.89 & 1.42 & 1.76 & 1.62 & 1.73 & 1.94 & 1.77 & 1.84 & 1.85 & 2.00 \\
\hline & 10 & 1.33 & 1.40 & 1.23 & 1.18 & 1.57 & 1.50 & 1.42 & 1.65 & 1.57 & 1.51 & 1.42 & 1.73 \\
\hline & 5 & 0.92 & 1.06 & 1.12 & 1.03 & 1.24 & 1.16 & 1.21 & 1.38 & 1.25 & 1.27 & 1.22 & 1.45 \\
\hline \multirow{5}{*}{6} & 25 & 3.97 & 3.82 & 3.60 & 3.56 & 4.18 & 3.99 & 4.09 & 3.74 & 4.24 & 4.06 & 3.94 & 3.80 \\
\hline & 20 & 3.40 & 3.19 & 3.14 & 3.07 & 3.55 & 3.29 & 3.28 & 3.18 & 3.48 & 3.45 & 3.39 & 3.25 \\
\hline & 15 & 3.06 & 2.95 & 2.89 & 2.73 & 3.15 & 3.13 & 3.04 & 2.84 & 3.25 & 3.18 & 2.98 & 2.99 \\
\hline & 10 & 2.43 & 2.22 & 2.32 & 2.20 & 2.55 & 2.34 & 2.46 & 2.99 & 2.61 & 2.44 & 2.48 & 3.14 \\
\hline & 5 & 1.84 & 1.77 & 1.88 & 1.50 & 1.97 & 1.87 & 1.80 & 1.62 & 1.93 & 1.83 & 1.73 & 1.79 \\
\hline \multirow{5}{*}{9} & 25 & 4.63 & 4.46 & 4.25 & 4.04 & 5.03 & 4.83 & 4.62 & 4.34 & 5.13 & 4.78 & 4.62 & 4.43 \\
\hline & 20 & 4.13 & 3.97 & 3.76 & 3.71 & 4.43 & 4.28 & 4.11 & 3.85 & 4.34 & 4.41 & 4.17 & 4.02 \\
\hline & 15 & 3.78 & 3.51 & 3.26 & 3.29 & 3.92 & 3.82 & 3.64 & 3.42 & 4.03 & 3.83 & 3.66 & 3.60 \\
\hline & 10 & 3.09 & 3.06 & 2.85 & 2.95 & 3.30 & 3.18 & 3.03 & 3.00 & 3.36 & 3.28 & 3.16 & 2.90 \\
\hline & 5 & 2.72 & 2.56 & 2.56 & 2.43 & 2.53 & 2.62 & 2.69 & 2.52 & 2.81 & 2.87 & 2.50 & 2.41 \\
\hline
\end{tabular}




\section{B.3 Medium Temperature Chiller}

The final performance map developed was that for this study was that of the medium temperature chiller. The data for compressor power consumption (Table B-7), the load side

(hot side) heat transfer rates (Table B-8) and source side (cold side) heat transfer rates (Table B-9) are provided in this section. This data was then formatted into input text files for the Type 927, which are shown in Appendix D. 
Table B-7: Electrical performance map for medium temperature chiller $(\mathrm{kW})$

\begin{tabular}{|c|c|c|c|c|c|c|c|c|c|c|c|c|c|c|c|c|}
\hline \multirow{2}{*}{$\begin{array}{l}\text { Flow Rate } \\
\text { (L/min) }\end{array}$} & \multirow{2}{*}{$\begin{array}{l}\text { Load Side } \\
\text { Temperature } \\
\left({ }^{\circ} \mathrm{C}\right)\end{array}$} & \multicolumn{5}{|c|}{10} & \multicolumn{5}{|c|}{15} & \multicolumn{5}{|c|}{20} \\
\hline & & 15 & 20 & 25 & 30 & 35 & 15 & 20 & 25 & 30 & 35 & 15 & 20 & 25 & 30 & 35 \\
\hline \multirow{7}{*}{5} & 25 & 3.68 & 4.01 & 4.32 & 4.65 & 4.97 & 2.91 & 3.26 & 3.59 & 3.95 & 4.29 & 2.57 & 2.90 & 3.17 & 3.52 & 3.82 \\
\hline & 20 & 3.46 & 3.85 & 4.17 & 4.59 & 4.95 & 2.86 & 3.20 & 3.50 & 3.85 & 4.17 & 2.49 & 2.82 & 3.14 & 3.46 & 3.78 \\
\hline & 15 & 3.23 & 3.65 & 3.98 & 4.44 & 4.82 & 2.74 & 3.10 & 3.39 & 3.77 & 4.09 & 2.44 & 2.75 & 3.05 & 3.36 & 3.66 \\
\hline & 10 & 3.17 & 3.55 & 3.83 & 4.27 & 4.60 & 2.65 & 2.97 & 3.27 & 3.61 & 3.92 & 2.33 & 2.66 & 2.95 & 3.31 & 3.62 \\
\hline & 5 & 2.98 & 3.38 & 3.67 & 4.14 & 4.48 & 2.56 & 2.88 & 3.20 & 3.52 & 3.84 & 2.26 & 2.58 & 2.91 & 3.23 & 3.55 \\
\hline & 0 & 2.79 & 3.22 & 3.51 & 4.01 & 4.37 & 2.47 & 2.79 & 3.08 & 3.41 & 3.71 & 2.19 & 2.51 & 2.83 & 3.15 & 3.48 \\
\hline & -5 & 2.62 & 3.07 & 3.34 & 3.87 & 4.23 & 2.38 & 2.69 & 2.98 & 3.30 & 3.60 & 2.11 & 2.43 & 2.76 & 3.08 & 3.41 \\
\hline \multirow{7}{*}{10} & 25 & 3.93 & 4.20 & 4.52 & 4.77 & 5.07 & 3.15 & 3.44 & 3.65 & 3.99 & 4.35 & 2.75 & 3.05 & 3.26 & 3.60 & 3.92 \\
\hline & 20 & 3.84 & 4.15 & 4.41 & 4.76 & 5.04 & 3.09 & 3.39 & 3.63 & 3.97 & 4.33 & 2.68 & 2.99 & 3.24 & 3.59 & 3.92 \\
\hline & 15 & 3.67 & 4.01 & 4.29 & 4.67 & 4.98 & 2.98 & 3.31 & 3.56 & 3.93 & 4.29 & 2.65 & 2.97 & 3.23 & 3.58 & 3.88 \\
\hline & 10 & 3.35 & 3.76 & 4.11 & 4.54 & 4.92 & 2.82 & 3.15 & 3.44 & 3.79 & 4.25 & 2.63 & 2.94 & 3.21 & 3.54 & 3.84 \\
\hline & 5 & 3.20 & 3.59 & 3.92 & 4.34 & 4.71 & 2.62 & 2.98 & 3.30 & 3.69 & 4.11 & 2.49 & 2.83 & 3.10 & 3.46 & 3.74 \\
\hline & 0 & 3.02 & 3.42 & 3.71 & 4.18 & 4.52 & 2.57 & 2.90 & 3.18 & 3.53 & 3.94 & 2.36 & 2.69 & 2.98 & 3.33 & 3.75 \\
\hline & -5 & 2.82 & 3.27 & 3.59 & 4.11 & 4.49 & 2.43 & 2.79 & 3.11 & 3.49 & 3.84 & 2.34 & 2.68 & 2.99 & 3.34 & 3.66 \\
\hline \multirow{7}{*}{15} & 25 & 3.83 & 4.14 & 4.52 & 4.80 & 5.14 & 3.07 & 3.39 & 3.68 & 4.01 & 4.33 & 2.76 & 3.04 & 3.26 & 3.56 & 3.92 \\
\hline & 20 & 3.82 & 4.13 & 4.42 & 4.74 & 5.04 & 3.07 & 3.39 & 3.65 & 4.00 & 4.31 & 2.72 & 3.02 & 3.23 & 3.57 & 3.89 \\
\hline & 15 & 3.79 & 4.10 & 4.36 & 4.70 & 4.98 & 3.05 & 3.37 & 3.61 & 3.96 & 4.29 & 2.69 & 2.99 & 3.21 & 3.55 & 3.87 \\
\hline & 10 & 3.66 & 3.99 & 4.26 & 4.63 & 4.93 & 2.97 & 3.31 & 3.57 & 3.95 & 4.24 & 2.66 & 2.96 & 3.19 & 3.52 & 3.85 \\
\hline & 5 & 3.37 & 3.74 & 4.12 & 4.50 & 4.88 & 2.86 & 3.19 & 3.49 & 3.83 & 4.17 & 2.57 & 2.88 & 3.14 & 3.48 & 3.80 \\
\hline & 0 & 3.10 & 3.51 & 3.84 & 4.28 & 4.64 & 2.58 & 2.94 & 3.30 & 3.65 & 4.05 & 2.38 & 2.70 & 3.02 & 3.34 & 3.72 \\
\hline & -5 & 3.08 & 3.49 & 3.81 & 4.27 & 4.63 & 2.62 & 2.97 & 3.31 & 3.66 & 4.01 & 2.39 & 2.72 & 3.02 & 3.37 & 3.69 \\
\hline
\end{tabular}


Table B-8: Load side heat transfer rate $(\mathrm{kW})$

\begin{tabular}{|c|c|c|c|c|c|c|c|c|c|c|c|c|c|c|c|c|}
\hline \multirow{2}{*}{$\begin{array}{l}\begin{array}{l}\text { Flow Rate } \\
\text { (L/min) }\end{array} \\
\text { Source } \\
\text { Side }\end{array}$} & \multirow{2}{*}{$\begin{array}{l}\text { Load Side } \\
\text { Temperature } \\
\left({ }^{\circ} \mathrm{C}\right)\end{array}$} & \multicolumn{5}{|c|}{10} & \multicolumn{5}{|c|}{15} & \multicolumn{5}{|c|}{20} \\
\hline & & 15 & 20 & 25 & 30 & 35 & 15 & 20 & 25 & 30 & 35 & 15 & 20 & 25 & 30 & 35 \\
\hline \multirow{7}{*}{5} & 25 & 11.1 & 10.9 & 10.7 & 10.5 & 10.3 & 11.5 & 11.3 & 11.3 & 11.1 & 11.0 & 11.3 & 11.2 & 11.1 & 10.9 & 10.8 \\
\hline & 20 & 10.4 & 10.3 & 10.1 & 10.1 & 10.0 & 10.7 & 10.6 & 10.4 & 10.4 & 10.3 & 10.6 & 10.5 & 10.4 & 10.2 & 10.1 \\
\hline & 15 & 9.4 & 9.5 & 9.4 & 9.5 & 9.5 & 9.9 & 9.8 & 9.6 & 9.6 & 9.4 & 9.5 & 9.5 & 9.6 & 9.5 & 9.5 \\
\hline & 10 & 9.1 & 9.0 & 8.8 & 8.7 & 8.6 & 9.1 & 9.0 & 8.9 & 8.8 & 8.8 & 8.7 & 8.6 & 8.9 & 8.7 & 8.8 \\
\hline & 5 & 8.2 & 8.3 & 8.0 & 8.2 & 8.1 & 8.4 & 8.3 & 8.3 & 8.1 & 8.0 & 7.9 & 7.9 & 8.2 & 8.1 & 8.3 \\
\hline & 0 & 7.6 & 7.6 & 7.4 & 7.6 & 7.5 & 7.6 & 7.5 & 7.4 & 7.3 & 7.3 & 7.0 & 7.1 & 7.4 & 7.3 & 7.5 \\
\hline & -5 & 6.9 & 7.0 & 6.7 & 7.0 & 7.0 & 6.8 & 6.7 & 6.7 & 6.6 & 6.5 & 6.1 & 6.2 & 6.6 & 6.6 & 6.9 \\
\hline \multirow{7}{*}{10} & 25 & 12.4 & 11.8 & 11.6 & 10.9 & 10.5 & 12.1 & 11.9 & 11.7 & 11.5 & 11.3 & 12.0 & 11.9 & 11.6 & 11.5 & 11.4 \\
\hline & 20 & 12.1 & 11.6 & 11.2 & 10.7 & 10.2 & 11.8 & 11.6 & 11.4 & 11.3 & 11.1 & 11.6 & 11.5 & 11.4 & 11.4 & 11.3 \\
\hline & 15 & 11.4 & 11.0 & 10.7 & 10.4 & 10.0 & 11.3 & 11.1 & 10.9 & 10.7 & 10.5 & 11.4 & 11.3 & 11.2 & 11.1 & 11.0 \\
\hline & 10 & 10.3 & 10.1 & 9.9 & 9.8 & 9.6 & 10.5 & 10.2 & 10.1 & 9.8 & 9.6 & 11.2 & 11.0 & 10.8 & 10.7 & 10.5 \\
\hline & 5 & 9.2 & 9.2 & 9.1 & 9.0 & 9.0 & 9.5 & 9.3 & 9.1 & 9.0 & 8.8 & 10.0 & 9.9 & 9.8 & 9.7 & 9.6 \\
\hline & 0 & 8.4 & 8.4 & 8.2 & 8.3 & 8.2 & 8.5 & 8.4 & 8.3 & 8.1 & 7.9 & 8.6 & 8.6 & 8.4 & 8.5 & 8.4 \\
\hline & -5 & 7.7 & 7.8 & 7.7 & 8.0 & 8.0 & 8.1 & 7.9 & 7.8 & 7.6 & 7.4 & 8.6 & 8.6 & 8.4 & 8.4 & 8.3 \\
\hline \multirow{7}{*}{15} & 25 & 12.0 & 11.6 & 11.5 & 10.9 & 10.6 & 12.0 & 11.8 & 11.8 & 11.5 & 11.4 & 11.9 & 11.8 & 11.7 & 11.5 & 11.4 \\
\hline & 20 & 11.9 & 11.5 & 11.2 & 10.8 & 10.4 & 11.8 & 11.7 & 11.5 & 11.3 & 11.2 & 11.6 & 11.5 & 11.4 & 11.3 & 11.2 \\
\hline & 15 & 11.7 & 11.3 & 10.8 & 10.5 & 10.1 & 11.5 & 11.4 & 11.2 & 11.1 & 10.9 & 11.4 & 11.3 & 11.1 & 11.0 & 10.9 \\
\hline & 10 & 11.3 & 10.9 & 10.5 & 10.1 & 9.8 & 11.2 & 11.1 & 10.9 & 10.7 & 10.6 & 11.1 & 11.0 & 10.8 & 10.7 & 10.6 \\
\hline & 5 & 10.3 & 10.0 & 9.8 & 9.5 & 9.3 & 10.7 & 10.4 & 10.3 & 9.9 & 9.7 & 10.6 & 10.4 & 10.2 & 10.0 & 9.8 \\
\hline & 0 & 9.0 & 8.8 & 8.7 & 8.5 & 8.4 & 9.3 & 9.1 & 8.9 & 8.7 & 8.5 & 9.3 & 9.1 & 8.9 & 8.8 & 8.6 \\
\hline & -5 & 9.0 & 8.8 & 8.6 & 8.5 & 8.3 & 9.3 & 9.1 & 8.9 & 8.7 & 8.5 & 9.3 & 9.2 & 8.9 & 8.8 & 8.5 \\
\hline
\end{tabular}


Table B-9: Source side heat transfer rate $(\mathrm{kW})$

\begin{tabular}{|c|c|c|c|c|c|c|c|c|c|c|c|c|c|c|c|c|}
\hline \multirow{2}{*}{$\begin{array}{l}\begin{array}{l}\text { Flow Rate } \\
\text { (L/min) }\end{array} \\
\text { Source } \\
\text { Side }\end{array}$} & \multirow{2}{*}{$\begin{array}{l}\text { Load Side } \\
\text { Temperature } \\
\left({ }^{\circ} \mathrm{C}\right)\end{array}$} & \multicolumn{5}{|c|}{10} & \multicolumn{5}{|c|}{15} & \multicolumn{5}{|c|}{20} \\
\hline & & 15 & 20 & 25 & 30 & 35 & 15 & 20 & 25 & 30 & 35 & 15 & 20 & 25 & 30 & 35 \\
\hline \multirow{7}{*}{5} & 25 & 8.14 & 7.64 & 7.08 & 6.61 & 6.08 & 8.24 & 7.96 & 7.70 & 7.40 & 7.13 & 8.18 & 8.06 & 7.85 & 7.77 & 7.60 \\
\hline & 20 & 7.42 & 7.05 & 6.58 & 6.26 & 5.84 & 7.66 & 7.31 & 6.90 & 6.57 & 6.19 & 7.85 & 7.55 & 7.12 & 6.89 & 6.53 \\
\hline & 15 & 6.67 & 6.25 & 5.89 & 5.43 & 5.04 & 6.96 & 6.70 & 6.18 & 6.05 & 5.66 & 7.87 & 7.34 & 6.76 & 6.25 & 5.70 \\
\hline & 10 & 5.37 & 5.34 & 4.86 & 5.05 & 4.80 & 6.13 & 5.91 & 5.56 & 5.41 & 5.12 & 7.15 & 6.71 & 6.07 & 5.72 & 5.18 \\
\hline & 5 & 5.03 & 4.81 & 4.82 & 4.49 & 4.38 & 5.33 & 5.18 & 4.93 & 4.84 & 4.64 & 6.06 & 5.70 & 5.26 & 4.94 & 4.54 \\
\hline & 0 & 4.05 & 4.01 & 3.98 & 3.93 & 3.90 & 4.65 & 4.53 & 4.19 & 4.17 & 3.94 & 5.94 & 5.40 & 4.74 & 4.26 & 3.66 \\
\hline & -5 & 3.22 & 3.27 & 3.35 & 3.39 & 3.45 & 3.92 & 3.83 & 3.50 & 3.54 & 3.33 & 5.45 & 4.85 & 4.12 & 3.58 & 2.91 \\
\hline \multirow{7}{*}{10} & 25 & 9.37 & 8.63 & 7.87 & 7.14 & 6.39 & 9.44 & 9.07 & 8.82 & 8.38 & 8.07 & 8.68 & 8.34 & 8.37 & 7.85 & 7.69 \\
\hline & 20 & 9.05 & 8.37 & 7.69 & 7.02 & 6.34 & 9.32 & 8.98 & 8.65 & 8.32 & 7.98 & 8.92 & 8.43 & 8.26 & 7.61 & 7.28 \\
\hline & 15 & 8.16 & 7.70 & 7.16 & 6.75 & 6.25 & 8.78 & 8.39 & 8.13 & 7.67 & 7.34 & 8.55 & 8.20 & 7.96 & 7.55 & 7.26 \\
\hline & 10 & 7.35 & 6.98 & 6.43 & 6.15 & 5.69 & 7.92 & 7.54 & 7.24 & 6.82 & 6.48 & 8.34 & 7.92 & 7.66 & 7.15 & 6.81 \\
\hline & 5 & 6.65 & 6.16 & 5.84 & 5.26 & 4.86 & 7.10 & 6.78 & 6.15 & 5.98 & 5.51 & 6.92 & 6.74 & 6.62 & 6.41 & 6.25 \\
\hline & 0 & 5.99 & 5.56 & 5.36 & 4.82 & 4.51 & 6.51 & 6.10 & 5.50 & 5.20 & 4.69 & 5.87 & 5.72 & 5.59 & 5.43 & 5.28 \\
\hline & -5 & 5.27 & 4.96 & 4.84 & 4.44 & 4.23 & 5.96 & 5.58 & 4.91 & 4.68 & 4.16 & 5.86 & 5.71 & 5.50 & 5.39 & 5.21 \\
\hline \multirow{7}{*}{15} & 25 & 8.94 & 8.33 & 7.85 & 7.17 & 6.63 & 9.28 & 8.98 & 8.79 & 8.44 & 8.20 & 9.26 & 9.06 & 9.08 & 8.77 & 8.68 \\
\hline & 20 & 8.86 & 8.29 & 7.52 & 7.06 & 6.39 & 9.00 & 8.72 & 8.45 & 8.17 & 7.89 & 9.13 & 8.91 & 8.74 & 8.50 & 8.31 \\
\hline & 15 & 8.63 & 8.11 & 7.23 & 6.91 & 6.21 & 8.81 & 8.50 & 8.19 & 7.88 & 7.58 & 8.97 & 8.72 & 8.50 & 8.24 & 8.01 \\
\hline & 10 & 8.44 & 7.86 & 7.09 & 6.61 & 5.94 & 8.66 & 8.35 & 7.93 & 7.68 & 7.32 & 8.93 & 8.67 & 8.26 & 8.08 & 7.75 \\
\hline & 5 & 7.39 & 7.01 & 6.51 & 6.18 & 5.74 & 8.05 & 7.66 & 7.35 & 6.92 & 6.57 & 8.32 & 8.01 & 7.66 & 7.39 & 7.06 \\
\hline & 0 & 6.19 & 5.89 & 5.60 & 5.31 & 5.02 & 6.86 & 6.53 & 6.20 & 5.87 & 5.54 & 7.19 & 6.86 & 6.42 & 6.14 & 5.76 \\
\hline & -5 & 6.24 & 5.95 & 5.53 & 5.31 & 4.96 & 6.94 & 6.57 & 6.17 & 5.82 & 5.43 & 7.35 & 7.00 & 6.43 & 6.19 & 5.73 \\
\hline
\end{tabular}




\section{Appendix C Experimental Uncertainty}

When taking experimental measurements and developing the experimental performance map, it is not only important to determine the measured values, but also the experimental uncertainty present in each reading. In the set-up, the flow meters and the power meter came with a factory determined uncertainty, while the uncertainty on the thermocouples was determined through experiment. The procedure and experimental uncertainty of the individual readings and calculate values are presented in this appendix.

\section{C.1 Temperature Uncertainty}

Type-T thermocouples were used throughout the experimental set-up, to determine the temperature profile within the tank, as well as the entering and exiting temperatures of the heat pump and the heat exchangers in the system. Thermocouples are custom built from a role of thermocouple wire, which comes with a standard uncertainty of $\pm 0.5^{\circ} \mathrm{C}$, however this is very conservative and over a very large temperature range $\left(-250^{\circ} \mathrm{C}\right.$ to $\left.350^{\circ} \mathrm{C}\right)$. Additional uncertainty can be introduced by the cold junction temperature, the uncertainty on voltage readings and the fitted polynomial. As these relate to the performance of the thermocouples under a wide range of conditions, while we are employing them under close to ideal conditions (steady room temperature cold junction, small temperature range, high accuracy 24 bit volt meter). As such, the experimental uncertainty on the thermocouple readings was determined experimentally.

To determine the uncertainty on the thermocouples, three representative thermocouples were built using wire from 3 different locations within the roll of thermocouple wire to ensure continuity throughout the roll. These were then installed into 
the National Instruments data acquisition system and the tips placed into a temperature bath, capable of holding the temperature within $\pm 0.02^{\circ} \mathrm{C}$. A high accuracy, RTD capable of measuring the fluid temperature to $\pm 0.02^{\circ} \mathrm{C}$ was also placed within the bath to give an accurate temperature reading. The bath was varied in $2^{\circ} \mathrm{C}$ increments from $5^{\circ} \mathrm{C}$ to $61^{\circ} \mathrm{C}$, and at each temperature set-point, readings were taken every 10 seconds for 20 minutes for each of the three thermocouples, giving a total of 10,440 individual measurements. Each of these were then compared to the RTD reading recorded at the same interval and the error on each of the readings for the three thermocouple, and these errors have been plotted in Figure C-1.

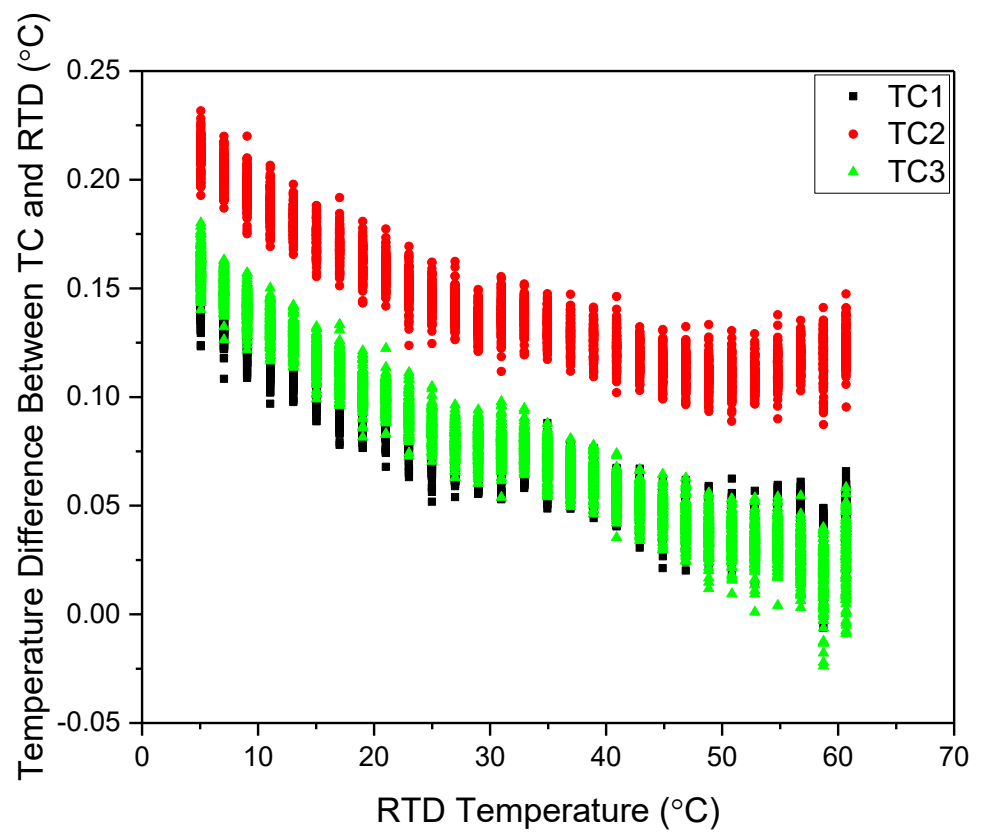

Figure C-1: Error on thermocouple readings

The average error was found to be $0.1^{\circ} \mathrm{C}$ and this was determined to be the bias in the equipment, as such, this value will be subtracted from all thermocouple readings both in 
these tests and when used in the experiments. Figure C-1 was then redrawn with these new errors, where the average error is now $0^{\circ} \mathrm{C}$, and is shown in Figure C-2.

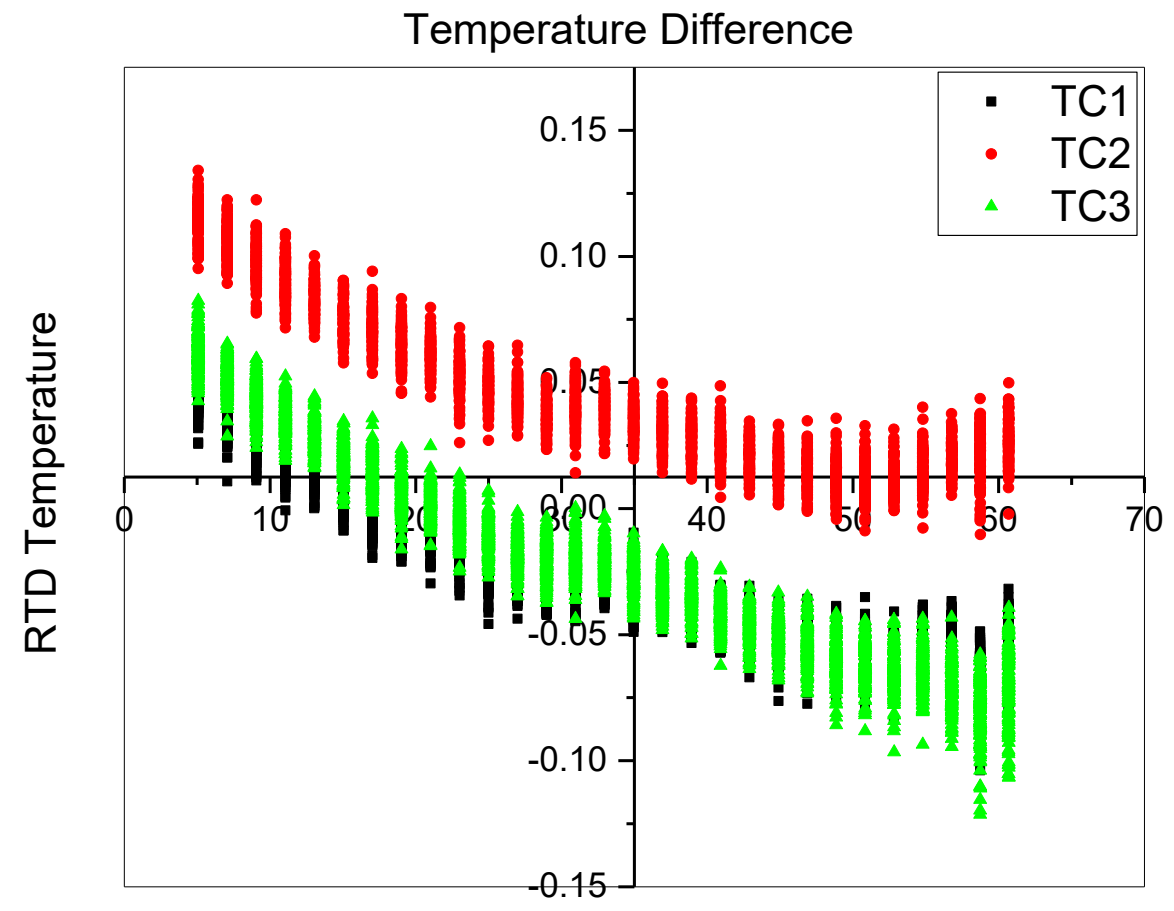

Figure C-2: Thermocouple error after removing equipment bias

From these values a standard deviation of $0.047^{\circ} \mathrm{C}$ was determined, and for a $99.9 \%$ confidence interval, an uncertainty of $\pm 0.16^{\circ} \mathrm{C}$ was found. To ensure a normal distribution 
was obtained, a histogram of the errors was plotted, along with a normal distribution trend line, as shown in Figure C-3.

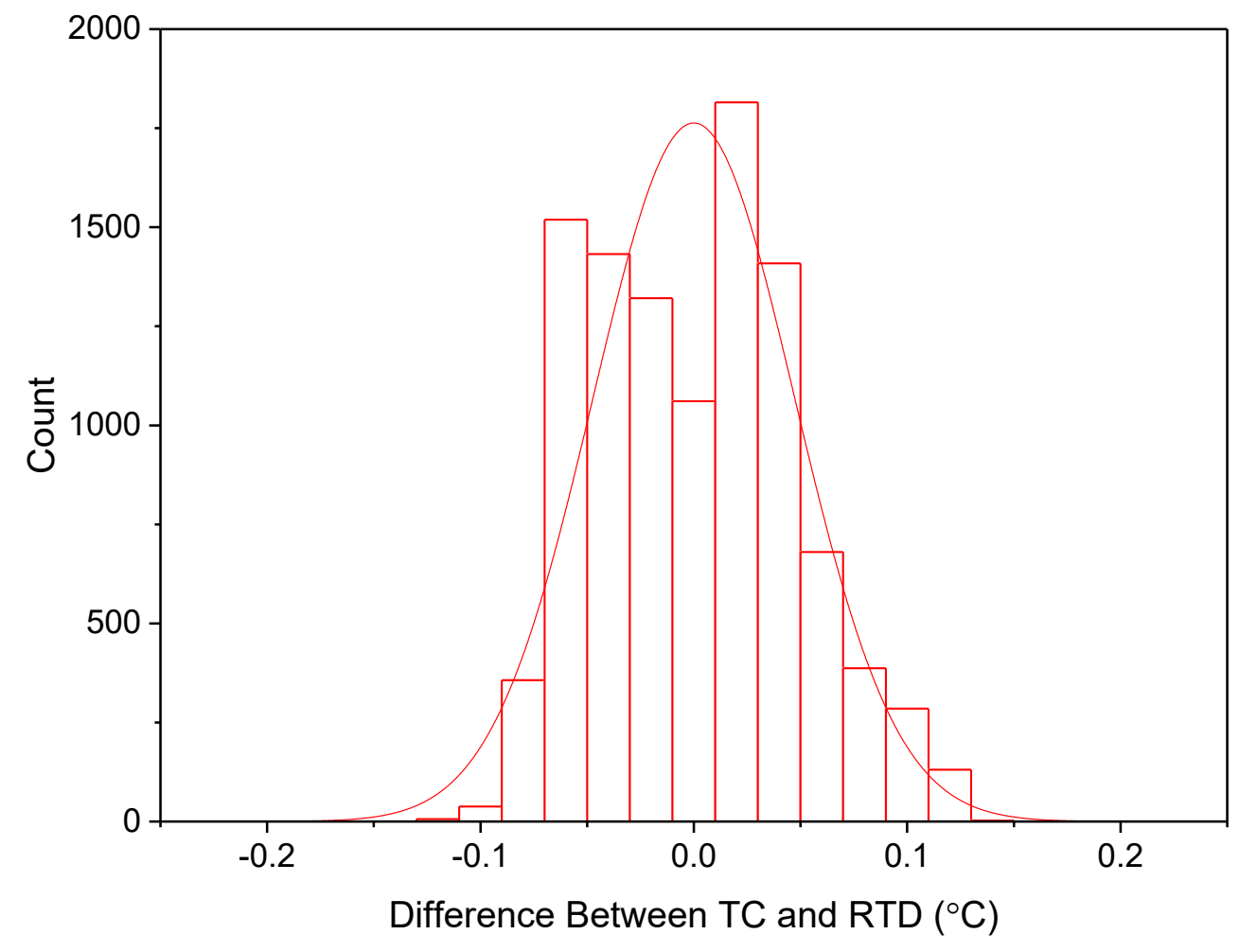

Figure C-3: Distribution of error on thermocouple readings

As the ambient conditions (most notably temperature) during the test can influence the results, this test was repeated two weeks later when ambient conditions were different, and similar results were obtained, with a final experimental uncertainty of $\pm 0.13^{\circ} \mathrm{C}$ and no measurements falling outside the previously obtained uncertainty of $\pm 0.15^{\circ} \mathrm{C}$, confirming 
the initial findings. The distribution of errors of the second test was plotted on the same graph as the distribution of errors on the first test and these are shown in Figure C-4.

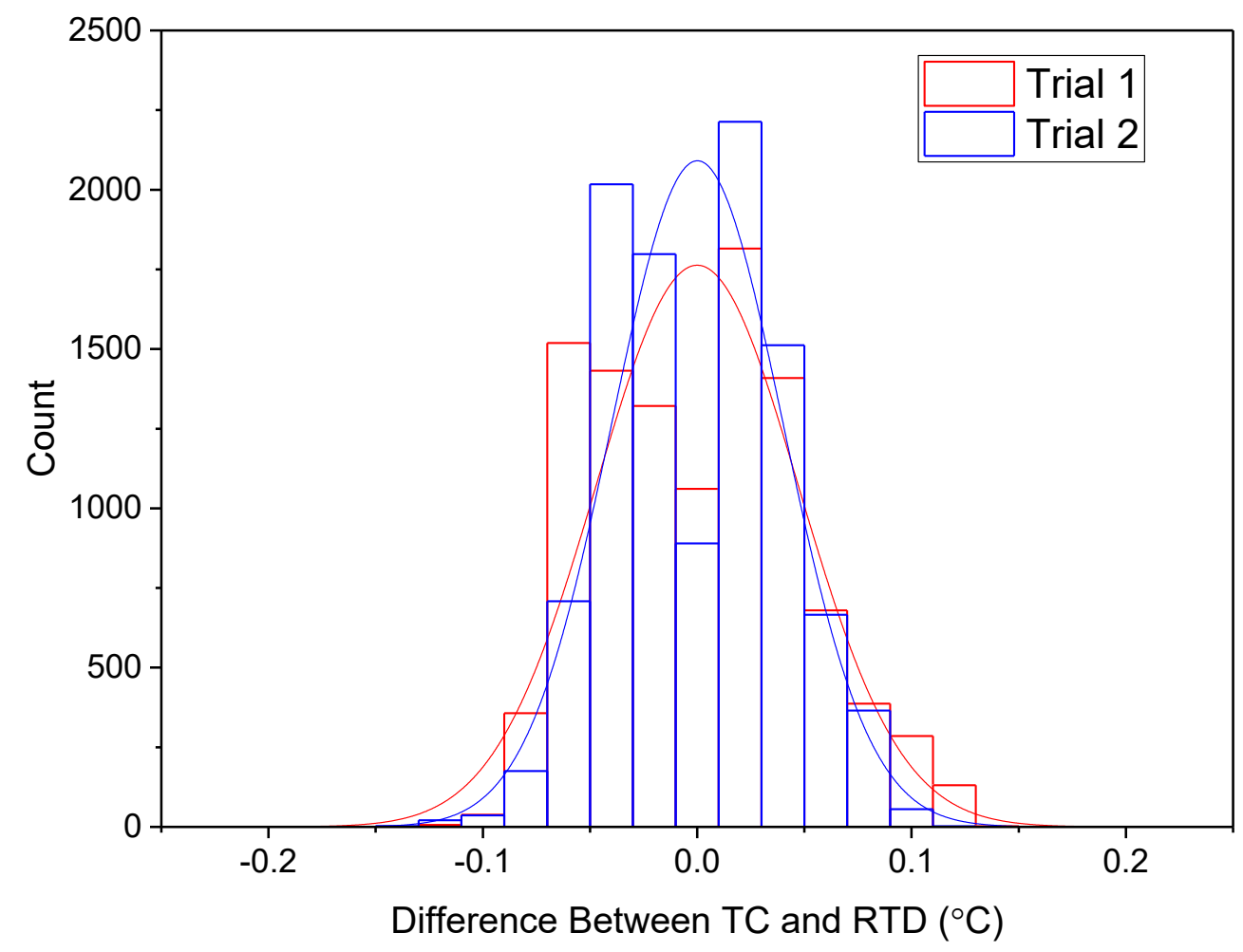

Figure C-4: Error distribution for both trials

To determine the overall uncertainty on the thermocouple readings, the uncertainty of the RTD and the bath uniformity (both $\pm 0.02^{\circ} \mathrm{C}$ ) must also be include. As these are all independent values, the root of the sum of the squares was used to determine the overall uncertainty of $\pm 0.16^{\circ} \mathrm{C}$ on all temperature measurements.

\section{C.2 Flow and Power Uncertainty}

The experimental uncertainty on the flow meter and power meter were both determined at the factory before being installed within the system. Unlike the temperature uncertainty, the uncertainty on the power meter and the flow meters in the system are in 
the form of a percentage of the reading, with both having an uncertainty of $1 \%$. Additionally, both the flow meter and power meter work off of producing a pulse every time a certain amount of fluid or energy passes through the meter. As such, in addition to the $1 \%$ uncertainty, an additional uncertainty of 1 pulse must be accounted for to take into consideration the quantity that has passed through the meter but not yet triggered a pulse. For the flow meter this added uncertainty is $\pm 0.003 \mathrm{~L}$, or if taken over a 30 second period, an uncertainty of $\pm 0.006 \mathrm{~L} / \mathrm{min}$, making the total experimental uncertainty on the flow rate $\pm(1 \%+0.006 \mathrm{~L} / \mathrm{min})$. The power meter produces a pulse for every $0.5 \mathrm{Wh}$, so when the power is taken over a 30 second period, this creates an uncertainty of $60 \mathrm{~W}$, however when the power is taken over a 10 minute period (the time used when developing the performance map), this uncertainty reduces to $3 \mathrm{~W}$. As such, for a 10 minute testing period, the overall experimental uncertainty on the power meter is $\pm(1 \%+3 \mathrm{~W})$.

\section{C.3 Uncertainty on Heat Transfer Rates}

Once the uncertainty on the measured values are determined, the overall uncertainty on calculated values can be determined. The heat transfer rate into or out of the heat pump are calculated using equation C-1.

$$
q=\dot{V} \rho c_{\mathrm{p}} \Delta T
$$

Using the values of uncertainty for each of the measured values, and the uncertainty on $\rho$ and $c_{p}$ found in Baldwin [73], the uncertainty on the hot and cold heat transfer rates were found and are shown in Table C-1 and C-2 for water, and the uncertainty on heat transfer rates with the glycol solution are shown in Table C-3 and C-4. This was completed through a propagation of uncertainty calculation, using the derivative with respect to each variable and then taking the root of squared sums to determine the overall uncertainty. 
Table C-1: Experimental uncertainty on the load side heat transfer rate $(\mathrm{kW})$ with water as the source side working fluid

\begin{tabular}{|c|c|c|c|c|c|c|c|c|c|c|c|c|c|c|c|c|}
\hline $\begin{array}{l}\text { Flow Rate } \\
\text { (L/min) }\end{array}$ & Load Side & \multicolumn{5}{|c|}{3} & \multicolumn{5}{|c|}{6} & \multicolumn{5}{|c|}{9} \\
\hline $\begin{array}{l}\text { Source } \\
\text { Side }\end{array}$ & $\begin{array}{l}\text { Temperature } \\
\left({ }^{\circ} \mathrm{C}\right)\end{array}$ & 20 & 25 & 30 & 35 & 40 & 20 & 25 & 30 & 35 & 40 & 20 & 25 & 30 & 35 & 40 \\
\hline \multirow{4}{*}{3} & 25 & 0.08 & 0.08 & 0.08 & 0.08 & 0.08 & 0.11 & 0.11 & 0.11 & 0.11 & 0.11 & 0.15 & 0.15 & 0.15 & 0.15 & 0.15 \\
\hline & 20 & 0.00 & 0.08 & 0.08 & 0.08 & 0.07 & 0.00 & 0.00 & 0.11 & 0.11 & 0.11 & 0.00 & 0.15 & 0.15 & 0.15 & 0.15 \\
\hline & 15 & 0.00 & 0.00 & 0.00 & 0.00 & 0.00 & 0.00 & 0.00 & 0.00 & 0.00 & 0.00 & 0.00 & 0.00 & 0.00 & 0.00 & 0.00 \\
\hline & 10 & 0.00 & 0.00 & 0.00 & 0.00 & 0.00 & 0.00 & 0.00 & 0.00 & 0.00 & 0.00 & 0.00 & 0.00 & 0.00 & 0.00 & 0.00 \\
\hline \multirow{4}{*}{6} & 25 & 0.08 & 0.09 & 0.09 & 0.09 & 0.00 & 0.12 & 0.12 & 0.11 & 0.12 & 0.11 & 0.16 & 0.16 & 0.16 & 0.16 & 0.00 \\
\hline & 20 & 0.08 & 0.08 & 0.08 & 0.08 & 0.00 & 0.12 & 0.12 & 0.11 & 0.11 & 0.11 & 0.15 & 0.16 & 0.00 & 0.16 & 0.00 \\
\hline & 15 & 0.08 & 0.08 & 0.08 & 0.08 & 0.00 & 0.11 & 0.11 & 0.11 & 0.11 & 0.11 & 0.16 & 0.16 & 0.15 & 0.15 & 0.00 \\
\hline & 10 & 0.08 & 0.07 & 0.07 & 0.07 & 0.00 & 0.11 & 0.11 & 0.11 & 0.11 & 0.11 & 0.15 & 0.15 & 0.15 & 0.15 & 0.00 \\
\hline \multirow{4}{*}{9} & 25 & 0.09 & 0.09 & 0.09 & 0.09 & 0.00 & 0.12 & 0.12 & 0.12 & 0.12 & 0.12 & 0.16 & 0.16 & 0.16 & 0.16 & 0.00 \\
\hline & 20 & 0.09 & 0.08 & 0.09 & 0.08 & 0.00 & 0.12 & 0.12 & 0.12 & 0.12 & 0.11 & 0.16 & 0.16 & 0.16 & 0.16 & 0.00 \\
\hline & 15 & 0.09 & 0.08 & 0.08 & 0.08 & 0.00 & 0.12 & 0.12 & 0.11 & 0.11 & 0.11 & 0.16 & 0.16 & 0.16 & 0.15 & 0.00 \\
\hline & 10 & 0.08 & 0.08 & 0.08 & 0.08 & 0.00 & 0.11 & 0.11 & 0.11 & 0.11 & 0.00 & 0.15 & 0.15 & 0.15 & 0.15 & 0.00 \\
\hline
\end{tabular}


Table C-2: Experimental uncertainty on the source side heat transfer rate $(\mathrm{kW})$ with water as the source side working fluid

\begin{tabular}{|c|c|c|c|c|c|c|c|c|c|c|c|c|c|c|c|c|}
\hline $\begin{array}{l}\text { Flow Rate } \\
\text { (L/min) }\end{array}$ & Load Side & \multicolumn{5}{|c|}{3} & \multicolumn{5}{|c|}{6} & \multicolumn{5}{|c|}{9} \\
\hline $\begin{array}{l}\text { Source } \\
\text { Side }\end{array}$ & $\begin{array}{l}\text { Temperature } \\
\left({ }^{\circ} \mathrm{C}\right)\end{array}$ & 20 & 25 & 30 & 35 & 40 & 20 & 25 & 30 & 35 & 40 & 20 & 25 & 30 & 35 & 40 \\
\hline \multirow{4}{*}{3} & 25 & 0.07 & 0.07 & 0.07 & 0.07 & 0.06 & 0.07 & 0.07 & 0.07 & 0.07 & 0.07 & 0.07 & 0.07 & 0.07 & 0.07 & 0.07 \\
\hline & 20 & 0.00 & 0.07 & 0.06 & 0.06 & 0.06 & 0.00 & 0.00 & 0.07 & 0.06 & 0.06 & 0.00 & 0.07 & 0.07 & 0.07 & 0.06 \\
\hline & 15 & 0.00 & 0.00 & 0.00 & 0.00 & 0.00 & 0.00 & 0.00 & 0.00 & 0.00 & 0.00 & 0.00 & 0.00 & 0.00 & 0.00 & 0.00 \\
\hline & 10 & 0.00 & 0.00 & 0.00 & 0.00 & 0.00 & 0.00 & 0.00 & 0.00 & 0.00 & 0.00 & 0.00 & 0.00 & 0.00 & 0.00 & 0.00 \\
\hline \multirow{4}{*}{6} & 25 & 0.11 & 0.11 & 0.11 & 0.00 & 0.00 & 0.11 & 0.11 & 0.11 & 0.11 & 0.11 & 0.12 & 0.11 & 0.11 & 0.11 & 0.00 \\
\hline & 20 & 0.11 & 0.11 & 0.11 & 0.11 & 0.00 & 0.11 & 0.11 & 0.11 & 0.11 & 0.11 & 0.11 & 0.11 & 0.00 & 0.11 & 0.00 \\
\hline & 15 & 0.11 & 0.11 & 0.10 & 0.10 & 0.00 & 0.11 & 0.11 & 0.11 & 0.10 & 0.10 & 0.11 & 0.11 & 0.11 & 0.10 & 0.00 \\
\hline & 10 & 0.10 & 0.10 & 0.10 & 0.10 & 0.00 & 0.11 & 0.10 & 0.10 & 0.10 & 0.10 & 0.11 & 0.10 & 0.10 & 0.10 & 0.00 \\
\hline \multirow{4}{*}{9} & 25 & 0.15 & 0.15 & 0.15 & 0.15 & 0.00 & 0.16 & 0.16 & 0.15 & 0.15 & 0.15 & 0.16 & 0.15 & 0.16 & 0.15 & 0.00 \\
\hline & 20 & 0.15 & 0.15 & 0.15 & 0.15 & 0.00 & 0.15 & 0.15 & 0.15 & 0.15 & 0.15 & 0.16 & 0.15 & 0.15 & 0.15 & 0.00 \\
\hline & 15 & 0.15 & 0.15 & 0.15 & 0.15 & 0.00 & 0.15 & 0.15 & 0.15 & 0.15 & 0.15 & 0.15 & 0.15 & 0.15 & 0.15 & 0.00 \\
\hline & 10 & 0.15 & 0.15 & 0.15 & 0.15 & 0.00 & 0.15 & 0.15 & 0.15 & 0.15 & 0.00 & 0.15 & 0.15 & 0.15 & 0.15 & 0.00 \\
\hline
\end{tabular}


Table C-3: Experimental uncertainty on the load side heat transfer rate $(\mathrm{kW})$ with a $50 / 50$ water/glycol solution by volume as the source side working fluid

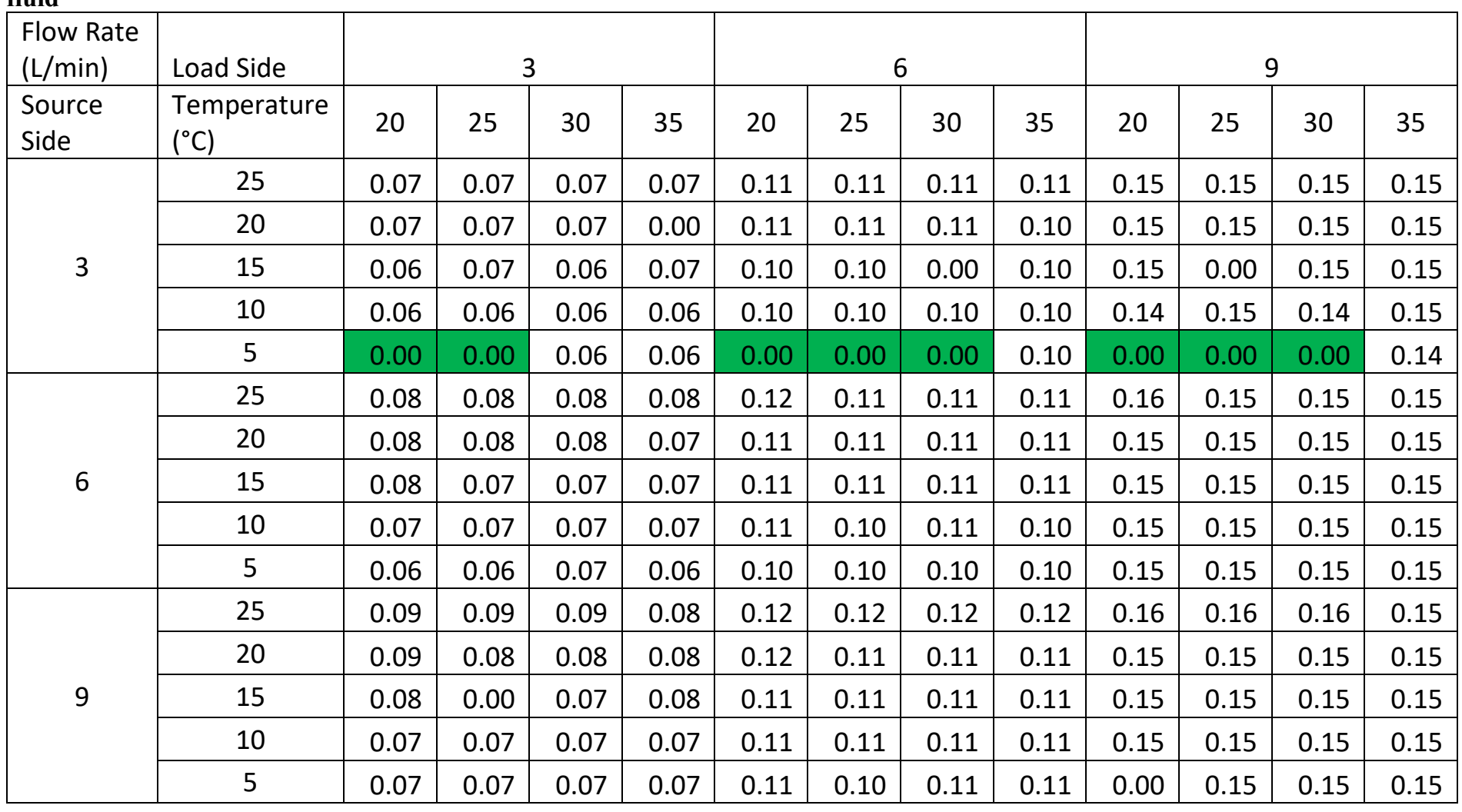


Table C-4: Experimental uncertainty on the source side heat transfer rate $(\mathrm{kW})$ with a $50 / 50$ water/glycol solution by volume as the source side working fluid

\begin{tabular}{|c|c|c|c|c|c|c|c|c|c|c|c|c|c|}
\hline \multirow{2}{*}{$\begin{array}{l}\text { Flow Rate } \\
\text { (L/min) } \\
\text { Source } \\
\text { Side }\end{array}$} & \multirow{2}{*}{$\begin{array}{l}\text { Load Side } \\
\text { Temperature } \\
\left({ }^{\circ} \mathrm{C}\right)\end{array}$} & \multicolumn{4}{|c|}{3} & \multicolumn{4}{|c|}{6} & \multicolumn{4}{|c|}{9} \\
\hline & & 20 & 25 & 30 & 35 & 20 & 25 & 30 & 35 & 20 & 25 & 30 & 35 \\
\hline \multirow{4}{*}{3} & 25 & 0.05 & 0.05 & 0.05 & 0.05 & 0.05 & 0.05 & 0.05 & 0.05 & 0.05 & 0.05 & 0.05 & 0.05 \\
\hline & 20 & 0.05 & 0.05 & 0.05 & 0.00 & 0.05 & 0.05 & 0.05 & 0.05 & 0.05 & 0.05 & 0.05 & 0.05 \\
\hline & 10 & 0.04 & 0.04 & 0.04 & 0.04 & 0.04 & 0.04 & 0.04 & 0.04 & 0.04 & 0.04 & 0.04 & 0.05 \\
\hline & 5 & 0.00 & 0.00 & 0.04 & 0.04 & 0.00 & 0.00 & 0.00 & 0.04 & 0.00 & 0.00 & 0.00 & 0.04 \\
\hline \multirow{2}{*}{6} & 25 & 0.09 & 0.09 & 0.09 & 0.09 & 0.09 & 0.09 & 0.09 & 0.09 & 0.09 & 0.09 & 0.09 & 0.09 \\
\hline & 20 & 0.09 & 0.09 & 0.09 & 0.09 & 0.09 & 0.09 & 0.09 & 0.09 & 0.09 & 0.09 & 0.09 & 0.09 \\
\hline \multirow{5}{*}{9} & 25 & 0.13 & 0.13 & 0.13 & 0.13 & 0.13 & 0.13 & 0.13 & 0.13 & 0.13 & 0.13 & 0.13 & 0.13 \\
\hline & 20 & 0.13 & 0.13 & 0.13 & 0.12 & 0.13 & 0.13 & 0.13 & 0.13 & 0.13 & 0.13 & 0.13 & 0.13 \\
\hline & 15 & 0.13 & 0.00 & 0.12 & 0.12 & 0.13 & 0.13 & 0.12 & 0.12 & 0.13 & 0.13 & 0.12 & 0.12 \\
\hline & 10 & 0.12 & 0.12 & 0.12 & 0.12 & 0.12 & 0.12 & 0.12 & 0.12 & 0.12 & 0.12 & 0.12 & 0.12 \\
\hline & 5 & 0.12 & 0.12 & 0.12 & 0.12 & 0.12 & 0.12 & 0.12 & 0.12 & 0.00 & 0.12 & 0.12 & 0.12 \\
\hline
\end{tabular}




\section{Appendix D TRNSYS Input files}

Once all of the data was experimentally obtained, TRNYS input files for the heat pump and medium temperature chiller were developed and are presented in this section.

\section{D.1 Heat Pump with a 50/50 water/glycol solution on the source side}

The below text was used as the input file for the standard, ground source heat pump using the 50/50 water/glycol solution. The first 4 lines set the parameters for the files, with the first two being the tested flow rates in $\mathrm{kg} / \mathrm{hr}$ and the next two lines being the tested source and load temperatures, while the remaining lines provide the compressor power and either the hot side or cold flow rates. Two separate files were developed, one that models the hot side flow rate and one the cold side flow rate as shown in Table D-1.

Table D-1: Text files for modelling the heat pump using a glycol/water solution on the cold side

\begin{tabular}{|c|c|c|c|c|c|c|c|c|}
\hline \multicolumn{4}{|c|}{ Cold Side File } & \multicolumn{5}{|c|}{ Hot Side File } \\
\hline 180 & 360 & 540 & & 180 & 360 & 540 & & \\
\hline 180 & 360 & 540 & & 189 & 376 & 567 & & \\
\hline 5 & 10 & 15 & 25 & 20 & 25 & 30 & 35 & \\
\hline & 25 & & & 5 & 10 & 15 & 20 & 25 \\
\hline \multicolumn{2}{|c|}{0.918968507} & \multicolumn{2}{|c|}{1.239370983} & \multicolumn{2}{|c|}{2.801335339} & \multicolumn{3}{|c|}{1.239370983} \\
\hline \multicolumn{2}{|c|}{1.062635767} & \multicolumn{2}{|c|}{1.333148028} & \multicolumn{2}{|c|}{3.304423696} & \multicolumn{3}{|c|}{1.272222222} \\
\hline \multirow{2}{*}{\multicolumn{2}{|c|}{1.12195938}} & \multicolumn{2}{|c|}{1.480909091} & \multicolumn{2}{|c|}{3.352456685} & \multicolumn{3}{|c|}{1.284297521} \\
\hline & & \multicolumn{2}{|c|}{1.68} & \multicolumn{2}{|c|}{4.048952543} & \multicolumn{3}{|c|}{1.318333333} \\
\hline \multicolumn{2}{|c|}{$\begin{array}{l}1.027533933 \\
1.328973136\end{array}$} & \multicolumn{2}{|c|}{1.272222222} & \multicolumn{2}{|c|}{4.358633399} & \multicolumn{3}{|c|}{1.35} \\
\hline \multicolumn{2}{|c|}{1.397624716} & & \multicolumn{2}{|c|}{2.812651214} & \multicolumn{3}{|c|}{1.333148028} \\
\hline \multicolumn{2}{|c|}{1.226037999} & 1.5 & & \multicolumn{2}{|c|}{3.187062555} & \multicolumn{3}{|c|}{1.365} \\
\hline \multicolumn{2}{|c|}{1.183177531} & \multicolumn{2}{|c|}{1.69} & \multicolumn{2}{|c|}{3.576415383} & \multicolumn{3}{|c|}{1.427472527} \\
\hline \multicolumn{2}{|c|}{1.458362615} & \multicolumn{2}{|c|}{1.284297521} & 3.93 & 304598 & 1.4 & & \\
\hline 1.48 & 71168 & 1.42 & & 4.32 & 238064 & 1.4 & 176471 & \\
\hline 1.89 & 305048 & 1.5 & & $2.7^{\prime}$ & 308508 & 1.4 & 909091 & \\
\hline 1.42 & 599273 & 1.7 & & 3.15 & 378425 & 1.5 & 666667 & \\
\hline 2.10 & 36169 & 1.3 & & 3.5 & 044057 & 1.5 & & \\
\hline 2.12 & 546979 & 1.4 & & $3.9^{\circ}$ & 426493 & 1.5 & 982759 & \\
\hline 1.94 & 362306 & 1.5 & & 4.22 & 697079 & 1.6 & 929329 & \\
\hline 1.40 & 719582 & 1.73 & & 2.83 & 12829 & 1.6 & & \\
\hline 2.27 & 371874 & 1.3 & & 3.13 & 57977 & 1.6 & & \\
\hline
\end{tabular}




\begin{tabular}{|c|c|c|c|}
\hline 2.157689579 & 1.491176471 & 3.453507732 & 1.74 \\
\hline 2.048986993 & 1.639929329 & 3.452261651 & 1.733333333 \\
\hline 2.040274415 & 1.730625 & 3.784349257 & 1.730625 \\
\hline 1.239611164 & 1.087389245 & 3.462948921 & 1.2825 \\
\hline 1.156377476 & 1.176867133 & 3.86156627 & 1.323157895 \\
\hline 1.2117 & 1.2878 & 5.482289566 & 1.36 \\
\hline 1.382734961 & 1.431428571 & 5.123401426 & 1.426666667 \\
\hline 1.56559523 & 1.090909091 & 5.375666148 & 1.457142857 \\
\hline 1.496967284 & 1.194 & 3.29874179 & 1.38 \\
\hline 1.416730584 & 1.316842105 & 3.756963968 & 1.458947368 \\
\hline 1.651873906 & 1.461818182 & 4.366675403 & 1.505217391 \\
\hline 1.75525388 & 1.112030075 & 4.764214216 & 1.539473684 \\
\hline 1.623569041 & 1.227272727 & 5.28965565 & 1.59375 \\
\hline 1.7277 & 1.3458 & 3.530818173 & 1.510909091 \\
\hline 1.94260155 & 1.49 & 3.854490823 & 1.594736842 \\
\hline 2.231746188 & 1.115 & 4.413347151 & 1.6375 \\
\hline 2.204832948 & 1.235 & 4.851449581 & 1.696216216 \\
\hline 2.143277081 & 1.373214286 & 4.919340381 & 1.735714286 \\
\hline 1.928281723 & 1.509292035 & 3.35698173 & 1.690434783 \\
\hline 2.407222013 & 1.119069767 & 3.787239202 & 1.758947368 \\
\hline 2.304748657 & 1.261538462 & 4.176267445 & 1.796666667 \\
\hline 2.137651309 & 1.403684211 & 4.442583922 & 1.826666667 \\
\hline 2.115960641 & 1.534054054 & 4.822977757 & 1.858666667 \\
\hline 1.251641069 & 1.045365728 & 4.215979481 & 1.341818182 \\
\hline 1.2675 & 1.1244 & 4.335801689 & 1.3825 \\
\hline 1.21555209 & 1.246353246 & 4.889927825 & 1.4136 \\
\hline 1.453732964 & 1.376470588 & 5.513046745 & 1.475172414 \\
\hline 1.574356641 & 1.052307692 & 5.895852184 & 1.5048 \\
\hline 1.510345621 & 1.14 & 3.797620372 & 1.44 \\
\hline 1.424596564 & 1.265454545 & 4.275302347 & 1.507058824 \\
\hline 1.725031014 & 1.396363636 & 4.78971 .5463 & \\
\hline 1.772314675 & 1.0546875 & 5.364368267 & 1.60125 \\
\hline 1.8375 & 1.1544 & 5.719885549 & 1.63875 \\
\hline 1.848481986 & 1.280869565 & 3.714917967 & 1.591764706 \\
\hline 2.003900951 & 1.421538462 & 4.090195155 & 1.635652174 \\
\hline 2.243720936 & 1.054054054 & 4.187852861 & 1.658571429 \\
\hline 2.248622999 & 1.17 & 5.259499199 & 1.746206897 \\
\hline 2.1808659 & 1.303030303 & 5.643254128 & 1.796449704 \\
\hline 2.091961575 & 1.439325843 & 3.762021802 & 1.721052632 \\
\hline 2.417745819 & 1.068571429 & 4.286878902 & 1.782857143 \\
\hline 2.323089393 & 1.185789474 & 4.681946203 & 1.881 \\
\hline 2.266570934 & 1.319072165 & 4.888243046 & 1.88164557 \\
\hline 2.149790837 & 1.45 & 4.962433203 & 1.87862069 \\
\hline 1.841119437 & 1.2825 & 2.998605845 & 1.087389245 \\
\hline 1.770277207 & 1.38 & 3.387050554 & 1.090909091 \\
\hline 1.876029032 & 1.510909091 & 3.697797081 & 1.112030075 \\
\hline
\end{tabular}




\begin{tabular}{|c|c|c|c|}
\hline 1.500958722 & 1.690434783 & 4.07874561 & 1.115 \\
\hline 2.433870053 & 1.323157895 & 4.4746865 & 1.119069767 \\
\hline 2.216562312 & 1.458947368 & 2.856765325 & 1.176867133 \\
\hline 2.323576198 & 1.594736842 & 3.267744122 & 1.194 \\
\hline 2.196794528 & 1.758947368 & 3.64278912 & 1.227272727 \\
\hline 3.06235032 & 1.36 & 4.143523854 & 1.235 \\
\hline 2.954667254 & 1.505217391 & 4.464746715 & 1.261538462 \\
\hline 2.89435638 & 1.6375 & 2.8499 & 1.2878 \\
\hline 2.731730618 & 1.796666667 & 3.245907005 & 1.316842105 \\
\hline 3.400542111 & 1.426666667 & 3.6749 & 1.3458 \\
\hline 3.18896236 & 1.539473684 & 4.138515013 & 1.373214286 \\
\hline 3.136375988 & 1.696216216 & 4.467093003 & 1.403684211 \\
\hline 3.065296879 & 1.826666667 & 2.894232048 & 1.431428571 \\
\hline 3.968242413 & 1.457142857 & 3.347581338 & 1.461818182 \\
\hline 3.820326912 & 1.59375 & 3.652980851 & 1.49 \\
\hline 3.598745249 & 1.735714286 & 3.788077067 & 1.509292035 \\
\hline 3.563676631 & 1.858666667 & 4.28433055 & 1.534054054 \\
\hline 1.974518425 & 1.1075 & 3.634024777 & 1.1075 \\
\hline 1.866664231 & 1.217142857 & 4.047506286 & 1.122857143 \\
\hline 1.798479961 & 1.340869565 & 4.56024272 & 1.137 \\
\hline 1.623785509 & 1.481052632 & 5.151399346 & 1.156666667 \\
\hline 2.550686806 & 1.122857143 & 5.636291187 & 1.168571429 \\
\hline 2.338373034 & 1.2384 & 4.143523854 & 1.217142857 \\
\hline 2.460266396 & 1.374782609 & 3.577261089 & 1.2384 \\
\hline 2.99266398 & 1.514117647 & 4.030744002 & 1.26375 \\
\hline 3.147990972 & 1.137 & 4.637502577 & 1.268108108 \\
\hline 3.129769895 & 1.26375 & 4.929522801 & 1.29 \\
\hline 3.037674164 & 1.396153846 & 3.571502774 & 1.340869565 \\
\hline 2.839674299 & 1.551428571 & 4.089222574 & 1.374782609 \\
\hline 3.549547913 & 1.156666667 & 4.513138574 & 1.396153846 \\
\hline 3.288160162 & 1.268108108 & 4.982799857 & 1.405714286 \\
\hline 3.279614348 & 1.405714286 & 5.572823182 & 1.432 \\
\hline 3.182852256 & 1.556756757 & 3.629095116 & 1.481052632 \\
\hline 4.184695118 & 1.168571429 & 4.025516656 & 1.514117647 \\
\hline 3.992561834 & 1.29 & 4.555668266 & 1.551428571 \\
\hline 4.089341167 & 1.432 & 4.921925554 & 1.556756757 \\
\hline 3.743819135 & 1.588695652 & 5.272198388 & 1.588695652 \\
\hline 1.929725767 & 1.06125 & 4.066906687 & 1.122159091 \\
\hline 1.8290049 & 1.17 & 4.545284287 & 1.143157895 \\
\hline 1.726732983 & 1.291428571 & 5.005701612 & 1.158 \\
\hline 1.791259258 & 1.415 & 5.706852956 & 1.171764706 \\
\hline 2.614560366 & 1.065137615 & 6.168344684 & 1.180540541 \\
\hline 2.440259621 & 1.171304348 & 3.979241853 & 1.24 \\
\hline 2.482039666 & 1.303846154 & 4.429420353 & 1.257 \\
\hline 3.135772324 & 1.434285714 & 5.073659703 & 1.29 \\
\hline 3.246517876 & 1.078676471 & 5.597284437 & 1.294285714 \\
\hline
\end{tabular}




\begin{tabular}{|c|c|c|c|}
\hline 3.178551212 & 1.195121951 & 6.060062772 & 1.302857143 \\
\hline 2.983802479 & 1.317272727 & 4.092714197 & 1.374 \\
\hline 2.990201949 & 1.465384615 & 4.473379633 & 1.397777778 \\
\hline 3.484059865 & 1.064516129 & 4.99565552 & 1.424347826 \\
\hline 3.445122746 & 1.191724138 & 5.468310691 & 1.428837209 \\
\hline 3.391194745 & 1.32 & 5.942219202 & 1.444137931 \\
\hline 3.250509641 & 1.474285714 & 4.103307235 & 1.514285714 \\
\hline 4.236043133 & 1.08 & 4.686710113 & 1.556842105 \\
\hline 4.060672905 & 1.196363636 & 4.893764736 & 1.572631579 \\
\hline 3.944338925 & 1.326486486 & 5.389384256 & 1.59 \\
\hline 3.79709251 & 1.48 & 5.702957591 & 1.585454545 \\
\hline 2.717117311 & 1.341818182 & 2.927467535 & 1.045365728 \\
\hline 2.562212263 & 1.44 & 3.344094705 & 1.052307692 \\
\hline 2.560111119 & 1.591764706 & 3.664303984 & 1.0546875 \\
\hline 2.432977998 & 1.721052632 & 4.047013479 & 1.054054054 \\
\hline 3.093115572 & 1.3825 & 4.497558324 & 1.068571429 \\
\hline 3.063057698 & 1.507058824 & 2.8879 & 1.1244 \\
\hline 2.849483939 & 1.635652174 & 3.276940077 & 1.14 \\
\hline 2.951081231 & 1.782857143 & 3.6579 & 1.1544 \\
\hline 3.77920622 & 1.4136 & 4.032955737 & 1.17 \\
\hline 3.51351 .5463 & & 4.436191401 & 1.185789474 \\
\hline 3.264979542 & 1.658571429 & 2.904402389 & 1.246353246 \\
\hline 3.293670736 & 1.881 & 3.29594725 & 1.265454545 \\
\hline 4.129344368 & 1.475172414 & 3.653140591 & 1.280869565 \\
\hline 3.966094686 & 1.60125 & 4.085892033 & 1.303030303 \\
\hline 3.762766461 & 1.746206897 & 4.436230312 & 1.319072165 \\
\hline 3.705577488 & 1.88164557 & 2.900362447 & 1.376470588 \\
\hline 4.630150437 & 1.5048 & 3.319984617 & 1.396363636 \\
\hline 4.463505146 & 1.63875 & 3.617233316 & 1.421538462 \\
\hline 4.247908028 & 1.796449704 & 3.901422723 & 1.439325843 \\
\hline 4.044834959 & 1.87862069 & 4.207519812 & 1.45 \\
\hline 2.526412717 & 1.122159091 & 3.495182264 & 1.06125 \\
\hline 2.624101702 & 1.24 & 4.112231984 & 1.065137615 \\
\hline 2.69207032 & 1.374 & 4.673192166 & 1.078676471 \\
\hline 2.524101799 & 1.514285714 & 4.981092957 & 1.064516129 \\
\hline 3.303835783 & 1.143157895 & 5.700297713 & 1.08 \\
\hline 3.178337081 & 1.257 & 3.458530775 & 1.17 \\
\hline 3.028260012 & 1.397777778 & 4.097660648 & 1.171304348 \\
\hline 3.004642756 & 1.556842105 & 4.585666721 & 1.195121951 \\
\hline 3.918386518 & 1.158 & 5.08774653 & 1.191724138 \\
\hline 3.820922113 & 1.29 & 5.61575305 & 1.196363636 \\
\hline 3.643335877 & 1.424347826 & 3.55526151 & 1.291428571 \\
\hline 3.418766462 & 1.572631579 & 4.144666588 & 1.303846154 \\
\hline 4.425582135 & 1.171764706 & 4.420341744 & 1.317272727 \\
\hline 4.279878713 & 1.294285714 & 5.04993915 & 1.32 \\
\hline 4.10512311 & 1.428837209 & 5.478589878 & 1.326486486 \\
\hline
\end{tabular}




\begin{tabular}{|ll|ll|}
\hline 3.851618151 & 1.59 & 3.642473655 & 1.415 \\
5.026955793 & 1.180540541 & 3.928267003 & 1.434285714 \\
4.829296132 & 1.302857143 & 4.522693877 & 1.465384615 \\
4.615400581 & 1.444137931 & 4.895554061 & 1.474285714 \\
4.342337313 & 1.585454545 & 5.355900231 & 1.48 \\
2.814087431 & 1.06970729 & 4.113512748 & 1.06970729 \\
2.869444499 & 1.172727273 & 4.668261787 & 1.066363636 \\
2.500386943 & 1.296923077 & 5.104162685 & 1.083157895 \\
2.412622976 & 1.4376 & 5.577690058 & 1.0725 \\
3.364801566 & 1.066363636 & 6.213660764 & 1.076470588 \\
3.283684391 & 1.191428571 & 3.953216464 & 1.172727273 \\
3.156079648 & 1.309565217 & 4.534810055 & 1.191428571 \\
2.900082391 & 1.455 & 4.98394623 & 1.194545455 \\
4.032731415 & 1.083157895 & 5.642027005 & 1.195862069 \\
3.834399781 & 1.194545455 & 5.76204401 & 1.172 \\
3.655008077 & 1.343076923 & 4.106274541 & 1.296923077 \\
3.604003133 & 1.4725 & 4.343808407 & 1.309565217 \\
4.344222509 & 1.0725 & 4.984636912 & 1.343076923 \\
4.409568296 & 1.195862069 & 5.551620533 & 1.33125 \\
4.166005753 & 1.33125 & 5.79412028 & 1.334545455 \\
4.015856998 & 1.482439024 & 4.043512548 & 1.4376 \\
5.134159685 & 1.076470588 & 4.365772595 & 1.455 \\
4.781609005 & 1.172 & 4.877738852 & 1.4725 \\
4.622450537 & 1.334545455 & 5.333708761 & 1.482439024 \\
4.428025571 & 1.475294118 & 5.671626205 & 1.475294118 \\
\hline
\end{tabular}

\section{D.2 Medium Temperature Chiller Input Files}

The second set of files developed were to model the low temperature chiller to be read in by Type 927, and are shown in Table D-2.

Table D-2: Input files for the medium temperature chiller

\begin{tabular}{|c|c|}
\hline Cold Side File & Hot Side File \\
\hline 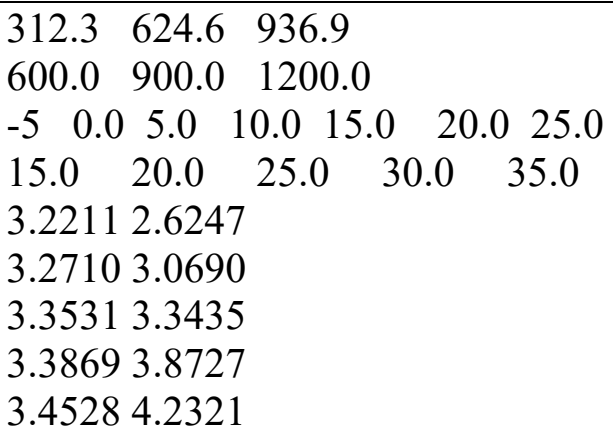 & 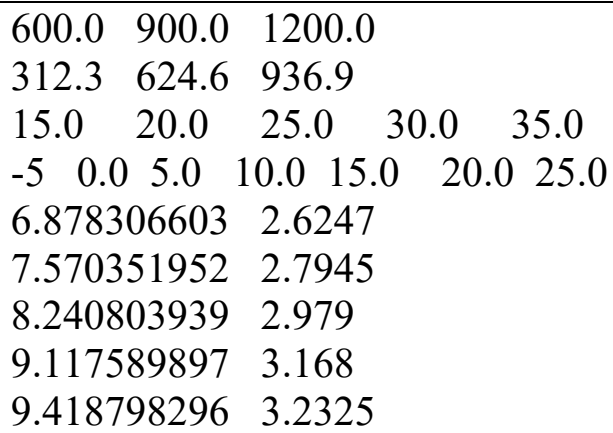 \\
\hline
\end{tabular}




\begin{tabular}{|c|c|}
\hline 4.04722 .7945 & $10.39084447 \quad 3.456$ \\
\hline 4.00743 .2238 & $11.0644034 \quad 3.684$ \\
\hline 3.97663 .5064 & $6.992837658 \quad 3.069$ \\
\hline 3.93234 .0091 & 7.640354988 \\
\hline 3.89704 .3650 & 8.270417285 \\
\hline 5.03002 .9790 & 9.007545439 \\
\hline 4.80983 .3840 & 9.471717736 \\
\hline 4.82403 .6720 & 10.30615554 \\
\hline 4.48674 .1355 & 10.85869888 \\
\hline 4.38374 .4820 & 6.72361176 \\
\hline 5.37083 .1680 & 7.394106656 \\
\hline 5.34083 .5505 & 7.999493464 \\
\hline 4.85753 .8340 & 8.80552687 \\
\hline 5.05424 .2660 & 9.408439548 \\
\hline 4.79764 .5990 & 10.11953091 \\
\hline 6.66973 .2325 & 10.69496592 \\
\hline 6.24643 .6495 & 7.030021291 \\
\hline 5.89113 .9825 & 7.622235375 \\
\hline 5.43374 .4415 & 8.179375394 \\
\hline 5.04444 .8165 & 8.741469468 \\
\hline 7.42103 .4560 & 9.519457801 \\
\hline 7.04803 .8475 & 10.08580983 \\
\hline 6.58344 .1670 & 10.46827563 \\
\hline 6.25634 .5945 & 6.95267387 \\
\hline 5.83754 .9500 & 7.534112727 \\
\hline 8.13513 .6840 & $8.058720157 \quad 4.482$ \\
\hline 7.63844 .0095 & $8.585437955 \quad 4.599$ \\
\hline 7.07824 .3200 & $9.514278427 \quad 4.8165$ \\
\hline 6.61314 .6530 & $9.950153055 \quad 4.95$ \\
\hline 6.08464 .9710 & 10.28355689 \\
\hline 3.91692 .3766 & 7.686858841 \\
\hline 3.83262 .6865 & 8.413490796 \\
\hline 3.50232 .9790 & $9.246429225 \quad 3.195$ \\
\hline 3.54103 .2976 & $10.26160292 \quad 3.3525$ \\
\hline 3.33373 .5988 & $11.37411757 \quad 3.6675$ \\
\hline 4.65352 .4689 & $12.08745523 \quad 3.8355$ \\
\hline 4.52722 .7855 & 12.36052974 \\
\hline 4.19043 .0812 & 7.830078505 \\
\hline 4.16943 .4083 & 8.402120569 \\
\hline 3.93783 .7145 & 9.172347369 \\
\hline 5.32572 .5590 & 10.11511801 \\
\hline 5.18152 .8800 & 11.04846173 \\
\hline 4.93233 .1950 & 11.59254539 \\
\hline 4.84053 .5190 & 11.82006054 \\
\hline 4.64373 .8370 & $7.74464146 \quad 3.5898$ \\
\hline 6.12902 .6490 & 8.227528987 \\
\hline
\end{tabular}




\begin{tabular}{|c|c|c|}
\hline 5.91232 .9745 & 9.1216049 & 3.924 \\
\hline 5.55683 .2670 & 9.903724895 & 4.1085 \\
\hline 5.40963 .6090 & 10.668995 & 4.2885 \\
\hline 5.12353 .9180 & 11.20086399 & 4.4055 \\
\hline 6.95682 .7435 & 11.56781378 & 4.5225 \\
\hline 6.69633 .0960 & 8.002189478 & 4.1085 \\
\hline 6.18413 .3885 & 8.297769437 & 4.176 \\
\hline 6.04963 .7710 & 9.035853352 & 4.3425 \\
\hline 5.66324 .0935 & 9.789694097 & 4.5405 \\
\hline 7.66172 .8635 & 10.37024461 & 4.671 \\
\hline 7.30713 .1995 & 10.65433993 & 4.7565 \\
\hline 6.89783 .4965 & 10.88323336 & 4.77 \\
\hline 6.57063 .8520 & 8.031080788 & 4.4926 \\
\hline 6.18874 .1685 & 8.204788532 & 4.521 \\
\hline 8.24212 .9130 & 8.97344119 & 4.707 \\
\hline 7.95683 .2625 & 9.610755085 & 4.9185 \\
\hline 7.70223 .5910 & 10.01768332 & 4.9815 \\
\hline 7.40163 .9510 & 10.21104431 & 5.0415 \\
\hline 7.13164 .2900 & 10.48687537 & 5.0685 \\
\hline 5.44902 .1066 & 9.005905142 & 3.0818 \\
\hline 4.84752 .4291 & 9.003441422 & 3.1035 \\
\hline 4.11622 .7612 & 10.28904422 & 3.366 \\
\hline 3.57973 .0789 & 11.28164223 & 3.6555 \\
\hline 2.91333 .4062 & 11.71417905 & 3.786 \\
\hline 5.94222 .1852 & 11.8973863 & 3.819 \\
\hline 5.40342 .5074 & 12.0030244 & 3.828 \\
\hline 4.73982 .8314 & 8.84571658 & 3.4911 \\
\hline 4.26343 .1527 & 8.846064715 & 3.5055 \\
\hline 3.66233 .4758 & 10.02190372 & 3.744 \\
\hline 6.06222 .2635 & 10.90309709 & 3.9915 \\
\hline 5.70122 .5830 & 11.32107626 & 4.0995 \\
\hline 5.25792 .9115 & 11.50168541 & 4.131 \\
\hline 4.93803 .2265 & 11.57166365 & 4.14 \\
\hline 4.53593 .5505 & 8.608468305 & 3.8142 \\
\hline 7.15072 .3295 & 8.694308078 & 3.8385 \\
\hline 6.70572 .6640 & 9.843788855 & 4.122 \\
\hline 6.06612 .9475 & 10.52667289 & 4.2615 \\
\hline 5.71843 .3075 & 10.83843523 & 4.356 \\
\hline 5.17613 .6165 & 11.19664203 & 4.419 \\
\hline 7.86822 .4435 & 11.45488418 & 4.518 \\
\hline 7.33792 .7495 & 8.486809599 & 4.2666 \\
\hline 6.75993 .0465 & 8.534121336 & 4.275 \\
\hline 6.25353 .3570 & 9.532135533 & 4.5 \\
\hline 5.69943 .6585 & 10.14706727 & 4.6305 \\
\hline 7.84792 .4945 & 10.49010156 & 4.698 \\
\hline 7.55292 .8170 & 10.7556124 & 4.743 \\
\hline
\end{tabular}




\begin{tabular}{|c|c|c|}
\hline 7.12093 .1365 & 10.86623279 & 4.797 \\
\hline 6.89443 .4605 & 8.288091181 & 4.6328 \\
\hline 6.53093 .7815 & 8.379554664 & 4.6425 \\
\hline 8.17942 .5740 & 9.30950785 & 4.878 \\
\hline 8.05682 .8980 & 9.769582601 & 4.9335 \\
\hline 7.84853 .1680 & 10.05222965 & 4.983 \\
\hline 7.76873 .5190 & 10.40524026 & 5.043 \\
\hline 7.60323 .8160 & 10.59216268 & 5.142 \\
\hline 5.26922 .8216 & 6.801061242 & 2.3766 \\
\hline 4.96303 .2730 & 7.581465315 & 2.46885 \\
\hline 4.84263 .5898 & 8.412740327 & 2.559 \\
\hline 4.44354 .1085 & 9.075838008 & 2.649 \\
\hline 4.23024 .4926 & 9.90644772 & 2.7435 \\
\hline 5.98903 .0180 & 10.73136385 & 2.8635 \\
\hline 5.56243 .4245 & 11.48699777 & 2.913 \\
\hline 5.36083 .7080 & 6.723051683 & 2.6865 \\
\hline 4.82174 .1760 & 7.495311621 & 2.7855 \\
\hline 4.50754 .5210 & 8.299739653 & 2.88 \\
\hline 6.64773 .1950 & 8.981962154 & 2.9745 \\
\hline 6.15553 .5865 & 9.805747678 & 3.096 \\
\hline 5.84473 .9240 & 10.64197455 & 3.1995 \\
\hline 5.26184 .3425 & 11.33103315 & 3.2625 \\
\hline 4.86034 .7070 & 6.68011054 & 2.979 \\
\hline 7.34743 .3525 & 7.438193101 & 3.08115 \\
\hline 6.97653 .7575 & 8.264569655 & 3.195 \\
\hline 6.42684 .1085 & 8.94446344 & 3.267 \\
\hline 6.14544 .5405 & 9.601381517 & 3.3885 \\
\hline 5.68514 .9185 & 10.44915024 & 3.4965 \\
\hline 8.15813 .6675 & 11.30263906 & 3.591 \\
\hline 7.70324 .0140 & 6.584566772 & 3.2976 \\
\hline 7.15774 .2885 & 7.33752182 & 3.4083 \\
\hline 6.74814 .6710 & 8.112653642 & 3.519 \\
\hline 6.24784 .9815 & 8.822399015 & 3.609 \\
\hline 9.05013 .8355 & 9.552514535 & 3.771 \\
\hline 8.37214 .1535 & 10.41147846 & 3.852 \\
\hline 7.69444 .4055 & 11.08288916 & 3.951 \\
\hline 7.01624 .7565 & 6.524091421 & 3.5988 \\
\hline 6.33845 .0415 & 7.265885712 & 3.71445 \\
\hline 9.36683 .9255 & 8.038568306 & 3.837 \\
\hline 8.62674 .1985 & 8.756711731 & 3.918 \\
\hline 7.87154 .5225 & 9.399981433 & 4.0935 \\
\hline 7.13894 .7700 & 10.27037165 & 4.1685 \\
\hline 6.39135 .0685 & 10.9907098 & 4.29 \\
\hline 5.95652 .4250 & 8.060503122 & 2.425 \\
\hline 5.58102 .7852 & 8.542023387 & 2.5695 \\
\hline 4.91483 .1146 & 9.467286212 & 2.622 \\
\hline
\end{tabular}




\begin{tabular}{|c|c|}
\hline 4.68473 .4902 & $10.46792754 \quad 2.817$ \\
\hline 4.16393 .8350 & $11.29996594 \quad 2.982$ \\
\hline 6.50542 .5695 & $11.77871172 \quad 3.087$ \\
\hline 6.10392 .8980 & 12.07958864 \\
\hline 5.49563 .1815 & $7.897229792 \quad 2.7852$ \\
\hline 5.19763 .5325 & $8.36579997 \quad 2.898$ \\
\hline 4.69263 .8385 & 9.3381398392 .9835 \\
\hline 7.09832 .6220 & $10.23716939 \quad 3.15$ \\
\hline 6.77742 .9835 & $11.12825387 \quad 3.312$ \\
\hline 6.14813 .3030 & $11.62775575 \quad 3.393$ \\
\hline 5.98133 .6855 & 11.87759032 \\
\hline 5.50614 .0260 & 7.7711895 \\
\hline 7.92022 .8170 & 8.291420993 \\
\hline 7.54033 .1500 & 9.106699229 \\
\hline 7.24423 .4380 & 10.10929934 \\
\hline 6.82243 .7935 & 10.87555931 \\
\hline 6.48444 .1040 & 11.39261647 \\
\hline 8.78272 .9820 & 11.72415715 \\
\hline 8.38883 .3120 & 7.589299651 \\
\hline 8.12893 .5550 & $8.064275356 \quad 3.5325$ \\
\hline 7.66813 .9285 & 9.028699975 \\
\hline 7.34124 .2150 & $9.827097128 \quad 3.7935$ \\
\hline 9.31683 .0870 & $10.74433848 \quad 3.9285$ \\
\hline 8.98393 .3930 & 11.283752153 .969 \\
\hline 8.64613 .6270 & 11.49787626 \\
\hline 8.31563 .9690 & 7.44464284 \\
\hline 7.98034 .2390 & 7.938974159 \\
\hline 9.44493 .1500 & 8.848406483 \\
\hline 9.06953 .4425 & 9.647783026 \\
\hline 8.81663 .6540 & $10.53213516 \quad 4.215$ \\
\hline 8.38003 .9870 & $11.09070453 \quad 4.239$ \\
\hline 8.06594 .2390 & $11.32016051 \quad 4.239$ \\
\hline 5.85852 .3376 & $9.336577328 \quad 2.6215$ \\
\hline 5.71252 .6754 & 9.252698286 \\
\hline 5.49772 .9892 & 10.67538264 \\
\hline 5.38623 .3390 & 11.24914739 \\
\hline 5.20583 .6648 & $11.54709502 \quad 3.0525$ \\
\hline 5.87302 .3565 & 11.81107826 \\
\hline 5.72122 .6865 & 12.025505893 .0675 \\
\hline 5.58782 .9835 & $9.113138791 \quad 2.9715$ \\
\hline 5.42683 .3300 & $9.060109421 \quad 2.9385$ \\
\hline 5.28433 .6435 & 10.40077178 \\
\hline 6.92162 .4945 & 11.09259731 \\
\hline 6.73942 .8260 & 11.39926842 \\
\hline 6.61883 .1005 & 11.65608079 \\
\hline 6.40573 .4605 & $11.83338217 \quad 3.3885$ \\
\hline
\end{tabular}




\begin{tabular}{|c|c|}
\hline 6.25443 .7635 & $8.936122645 \quad 3.3069$ \\
\hline 8.34212 .6280 & $8.932540592 \quad 3.2985$ \\
\hline 7.91522 .9385 & 10.25358725 \\
\hline 7.65883 .2130 & 10.87002269 \\
\hline 7.14683 .5415 & 11.19376418 \\
\hline 6.80513 .8340 & 11.47381786 \\
\hline 8.55152 .6505 & 11.75697925 \\
\hline 8.19852 .9700 & 8.689472912 \\
\hline 7.96433 .2265 & 8.707441709 \\
\hline 7.55193 .5775 & 9.915263227 \\
\hline 7.25833 .8655 & 10.74648488 \\
\hline 8.92162 .6775 & 11.074776413 .96 \\
\hline 8.43372 .9925 & $11.33245313 \quad 4.0005$ \\
\hline 8.25733 .2445 & 11.50699513 \\
\hline 7.61373 .5910 & 8.489245571 \\
\hline 7.28153 .8745 & $8.547362863 \quad 4.0065$ \\
\hline 8.67612 .7540 & 9.704365536 \\
\hline 8.33833 .0510 & 10.55692253 \\
\hline 8.36603 .2580 & 10.898111 \\
\hline 7.84553 .6000 & $11.16382294 \quad 4.2885$ \\
\hline 7.69043 .8520 & $11.37273181 \quad 4.3185$ \\
\hline 6.23713 .0818 & $6.096152346 \quad 2.1066$ \\
\hline 5.95353 .4911 & $6.975052882 \quad 2.1852$ \\
\hline 5.52573 .8142 & $7.886604052 \quad 2.2635$ \\
\hline 5.31414 .2666 & $8.693221918 \quad 2.3295$ \\
\hline 4.95844 .6328 & $9.547162705 \quad 2.4435$ \\
\hline 6.18773 .1035 & $10.60813218 \quad 2.4945$ \\
\hline 5.89443 .5055 & 11.3236516 \\
\hline 5.60283 .8385 & 6.220991258 \\
\hline 5.30874 .2750 & 7.051586219 \\
\hline 5.01624 .6425 & $7.932986604 \quad 2.583$ \\
\hline 7.39183 .3660 & $8.645438726 \quad 2.664$ \\
\hline 7.00523 .7440 & $9.496742329 \quad 2.7495$ \\
\hline 6.51094 .1220 & $10.46601416 \quad 2.817$ \\
\hline 6.17814 .5000 & 11.175673692 .898 \\
\hline 5.73774 .8780 & $6.640471486 \quad 2.7612$ \\
\hline 8.43843 .6555 & 7.387451466 \\
\hline 7.86203 .9915 & 8.16039981 \\
\hline 7.09154 .2615 & 8.859876618 \\
\hline 6.61214 .6305 & 9.6158303123 .0465 \\
\hline 5.93874 .9335 & $10.36122455 \quad 3.1365$ \\
\hline 8.62533 .7860 & $11.14462575 \quad 3.168$ \\
\hline 8.11494 .0995 & 6.6179897393 .0789 \\
\hline 7.23394 .3560 & $7.334318847 \quad 3.1527$ \\
\hline 6.90874 .6980 & $8.116267035 \quad 3.2265$ \\
\hline 6.21304 .9830 & $8.680982884 \quad 3.3075$ \\
\hline
\end{tabular}




\begin{tabular}{|c|c|}
\hline 8.85863 .8190 & $9.480655756 \quad 3.357$ \\
\hline 8.29454 .1310 & $10.20044232 \quad 3.4605$ \\
\hline 7.52314 .4190 & $10.93818286 \quad 3.519$ \\
\hline 7.06264 .7430 & $6.890149309 \quad 3.4062$ \\
\hline 6.39485 .0430 & $7.540518139 \quad 3.4758$ \\
\hline 8.94493 .8280 & $8.253164914 \quad 3.5505$ \\
\hline 8.33054 .1400 & 8.764310234 \\
\hline 7.85444 .5180 & 9.51498956 \\
\hline 7.17084 .7970 & 10.07698851 \\
\hline 6.62565 .1420 & $10.84866993 \quad 3.816$ \\
\hline 6.93652 .6215 & $8.597478314 \quad 2.3376$ \\
\hline 6.56852 .9715 & $8.616819724 \quad 2.3565$ \\
\hline 6.16683 .3069 & $9.982426971 \quad 2.4945$ \\
\hline 5.81583 .6642 & $11.16115857 \quad 2.628$ \\
\hline 5.43104 .0069 & $11.39645167 \quad 2.6505$ \\
\hline 6.86372 .5845 & $11.56408462 \quad 2.6775$ \\
\hline 6.53372 .9385 & $11.99918561 \quad 2.754$ \\
\hline 6.20023 .2985 & $8.570310497 \quad 2.6754$ \\
\hline 5.87203 .6495 & 8.6354064492 .6865 \\
\hline 5.54034 .0065 & $9.895153194 \quad 2.826$ \\
\hline 8.04892 .8605 & $10.99351621 \quad 2.9385$ \\
\hline 7.65873 .1860 & $11.30247394 \quad 2.97$ \\
\hline 7.34993 .4875 & $11.5170933 \quad 2.9925$ \\
\hline 6.91893 .8250 & $11.86156631 \quad 3.051$ \\
\hline 6.56944 .1385 & 8.3955709432 .9892 \\
\hline 8.65842 .9715 & $8.393028177 \quad 2.9835$ \\
\hline 8.35263 .3120 & $9.805099548 \quad 3.1005$ \\
\hline 7.92863 .5685 & 10.82373577 \\
\hline 7.68203 .9510 & $11.17157774 \quad 3.2265$ \\
\hline 7.31724 .2495 & $11.43915573 \quad 3.2445$ \\
\hline 8.80773 .0525 & $11.64412477 \quad 3.258$ \\
\hline 8.49863 .3660 & $8.442188994 \quad 3.339$ \\
\hline 8.19463 .6135 & 8.5420974 \\
\hline 7.88283 .9600 & $9.719215707 \quad 3.4605$ \\
\hline 7.57634 .2405 & $10.65716245 \quad 3.5415$ \\
\hline 8.99803 .0735 & $11.09605923 \quad 3.5775$ \\
\hline 8.72053 .3930 & $11.40763754 \quad 3.591$ \\
\hline 8.44513 .6495 & $11.54641659 \quad 3.6$ \\
\hline 8.16674 .0005 & $8.341235309 \quad 3.6648$ \\
\hline 7.89034 .2885 & $8.430201626 \quad 3.6435$ \\
\hline 9.27553 .0675 & $9.630551995 \quad 3.7635$ \\
\hline 8.97753 .3885 & $10.48845105 \quad 3.834$ \\
\hline 8.79363 .6765 & $10.98362226 \quad 3.8655$ \\
\hline 8.43864 .0140 & $11.3451731 \quad 3.8745$ \\
\hline 8.19774 .3185 & $11.36888617 \quad 3.852$ \\
\hline 7.34942 .3870 & $9.339892333 \quad 2.387$ \\
\hline
\end{tabular}




\begin{tabular}{|c|c|}
\hline 6.99852 .7186 & $9.278153461 \quad 2.376$ \\
\hline 6.43243 .0222 & 10.616562892 .5695 \\
\hline 6.18923 .3678 & 11.09040653 \\
\hline 5.73063 .6854 & 11.38229773 \\
\hline 7.18742 .3760 & 11.61642256 \\
\hline 6.85582 .7000 & 11.90203371 \\
\hline 6.41993 .0150 & 9.153585616 \\
\hline 6.14063 .3435 & 9.112223549 \\
\hline 5.75683 .6630 & 10.39845204 \\
\hline 8.31562 .5695 & 10.9787886 \\
\hline 8.01482 .8845 & 11.25957608 \\
\hline 7.65793 .1365 & 11.51170012 \\
\hline 7.38513 .4830 & 11.75178915 \\
\hline 7.05623 .7665 & 8.912555028 \\
\hline 8.93032 .6565 & $8.888010774 \quad 3.015$ \\
\hline 8.67422 .9565 & $10.22915974 \quad 3.1365$ \\
\hline 8.26023 .1905 & 10.82931669 \\
\hline 8.08313 .5235 & 11.12078056 \\
\hline 7.74803 .7905 & 11.40673583 \\
\hline 8.96882 .6865 & 11.68409523 \\
\hline 8.72412 .9880 & 8.753610246 \\
\hline 8.49763 .2085 & 8.751222294 \\
\hline 8.24373 .5505 & $9.986639604 \quad 3.483$ \\
\hline 8.00813 .8115 & $10.73662575 \quad 3.5235$ \\
\hline 9.12552 .7195 & $11.00609586 \quad 3.5505$ \\
\hline 8.91043 .0195 & $11.30213431 \quad 3.573$ \\
\hline 8.73773 .2265 & 11.49257534 \\
\hline 8.50153 .5730 & 8.539941594 \\
\hline 8.30763 .8265 & 8.55615095 \\
\hline 9.25622 .7630 & 9.792938028 \\
\hline 9.05683 .0375 & 10.60608083 \\
\hline 9.07623 .2580 & 10.87533728 \\
\hline 8.76743 .5595 & 11.19729095 \\
\hline 8.67743 .8070 & 11.3836061 \\
\hline
\end{tabular}




\section{Appendix E Source Code for the Ice Storage}

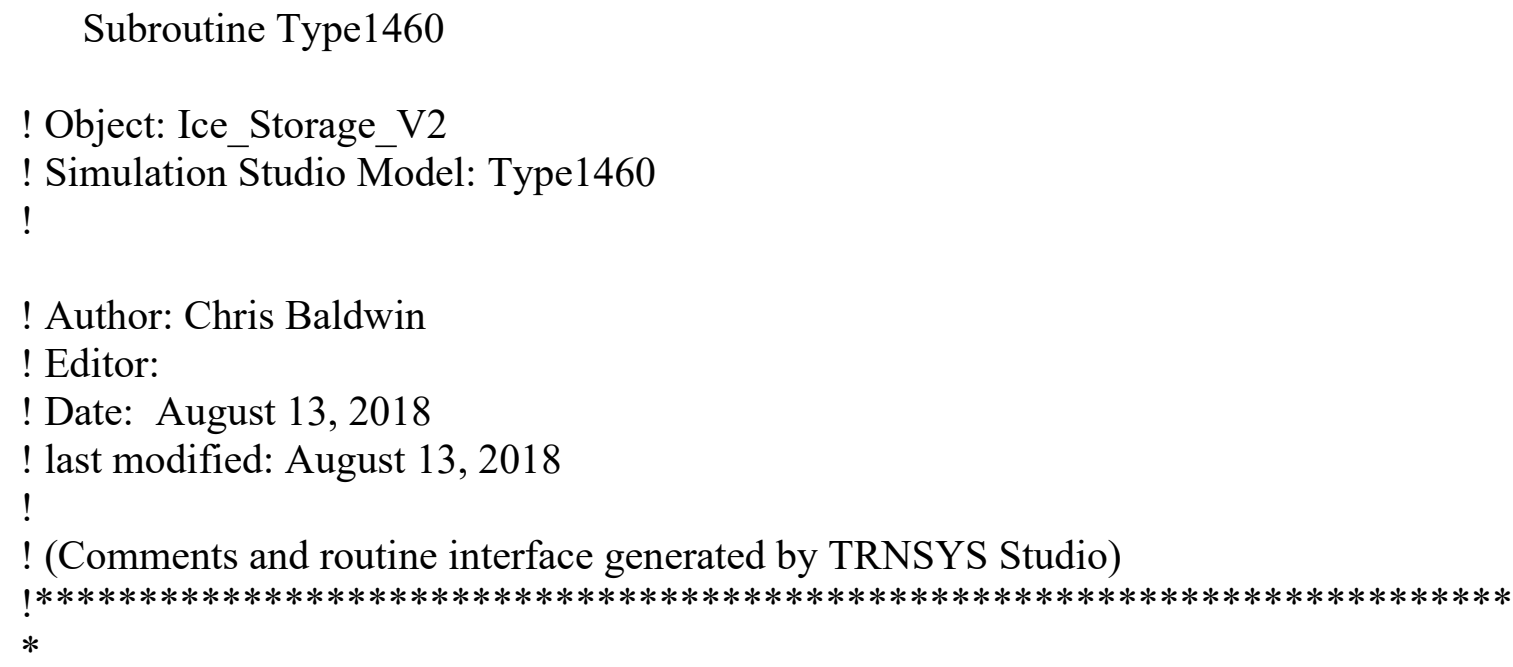

Subroutine Type 1460

! Object: Ice_Storage_V2

! Simulation Studio Model: Type1460

!

! Author: Chris Baldwin

! Editor:

! Date: August 13, 2018

! last modified: August 13, 2018

!

! (Comments and routine interface generated by TRNSYS Studio)

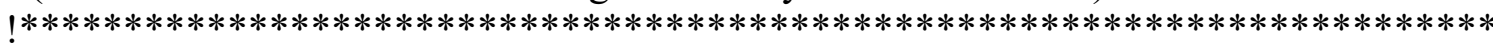

$*$

Use TrnsysConstants

Use TrnsysFunctions

!DEC\$Attributes DLLexport :: Type1460

!Trnsys Declarations

Implicit None

Double Precision Timestep,Time

Integer CurrentUnit,CurrentType

! PARAMETER

!Working Fluid Propoerties

DOUBLE PRECISION rho_glycol

DOUBLE PRECISION k_glycol

DOUBLE PRECISION Nu_glycol

DOUBLE PRECISION cp_glycol

!Tank Fluid Properties

DOUBLE PRECISION rho_water 
DOUBLE PRECISION k_water DOUBLE PRECISION Beta_water DOUBLE PRECISION alpha water DOUBLE PRECISION visc_water DOUBLE PRECISION cp_water !Pipe Properties DOUBLE PRECISION k pipe DOUBLE PRECISION i Diameter DOUBLE PRECISION t_wall DOUBLE PRECISION L_pipe DOUBLE PRECISION nodes pipe !Tank Properties DOUBLE PRECISION Volume_Fluid DOUBLE PRECISION Surface_Area DOUBLE PRECISION k_tank_wall DOUBLE PRECISION T $T^{-}$Water int DOUBLE PRECISION T_surroundings DOUBLE PRECISION rows DOUBLE PRECISION columns DOUBLE PRECISION V9

!Ice Properties DOUBLE PRECISION $\mathrm{k}$ ice DOUBLE PRECISION $\mathrm{hfg}$ _ice DOUBLE PRECISION rho ice DOUBLE PRECISION V1 $\overline{3}$ DOUBLE PRECISION V14 DOUBLE PRECISION V15

\section{! INPUTS}

DOUBLE PRECISION mdot_glycol DOUBLE PRECISION T_glycol_in DOUBLE PRECISION mdot_water DOUBLE PRECISION T water in DOUBLE PRECISION T_Ref

!Local Variables

Integer $\mathrm{i}, \mathrm{j}, \mathrm{k}, \mathrm{m}$, nodes, $\mathrm{h}, \mathrm{f}$

Double Precision n, C, itteration, Li, Pi, q, R1, R2, R3, R4, Ra, R_Total, T1_in, T1_out, T2_in, T2_out, Ts, T_tank, Volume_ice, Volume_displaced, Volume, thick_tank, delta_mass

Double Precision T_Tank_Int, T_Tank_test, Tout i, Tin_i, T3_in, T3_out, Tank_Volume_New, r_o_pipe, q_total, q_loss, q_i, delta_T_tank, energy, e_loss, e_zero, mass_ice, e_i

Double Precision total_mass_ice, Total_Volume_Ice, total_delta_mass_ice, total_pipes, sensible, latent, mdot_glycol_pipe, q_stored_total, q_out, energy_out, net_energy 
Double Precision mass_melted, T_energy_A_ice, mass_melted_node, T_Film, DT, Energy_Ice_Int, Energy_Sensible, Ice_After_Sensible, Net_Energy_pipe, Percent_Ice_E

Double Precision, dimension(12):: q_array, T_out_array, t_ice_array, energy_array, delta_mass_array,delta_volume_array,mass_ice_array,volume_ice_array, energy_after_ice

Double Precision, dimension(12):: T_Surface_Array, Mass_Ice_array_diff, delta_mass_array_diff, energy_A_ice

!Get the Global Trnsys Simulation Variables

Time $=$ getSimulationTime ()

Timestep $=$ getSimulationTimeStep ()

CurrentUnit $=$ getCurrentUnit()

CurrentType $=$ getCurrentType ()

!Set the Version Number for This Type

If(getIsVersionSigningTime()) Then

Call SetTypeVersion(17)

Return

EndIf

!--

$1-$

!Do Any Last Call Manipulations Here

If(getIsLastCallofSimulation()) Then

Return

EndIf

!Perform Any "After Convergence" Manipulations That May Be Required at the End of Each Timestep

If(getIsEndOfTimestep()) Then 
EndIf

Return

Endif

!-

!Do All of the "Very First Call of the Simulation Manipulations" Here If(getIsFirstCallofSimulation()) Then

!Tell the TRNSYS Engine How This Type Works

the the model wants

Call SetNumberofParameters(25) !The number of parameters that

Call SetNumberofInputs(5) !The number of inputs that the the

model wants

Call SetNumberofDerivatives(0) !The number of derivatives that the

the model wants

Call SetNumberofOutputs(25) !The number of outputs that the

the model produces

Call SetIterationMode(1)

!An indicator for the iteration mode (default=1). Refer to section 8.4.3.5 of the documentation for more details.

Call SetNumberStoredVariables $(0,100) \quad$ !The number of static variables that the model wants stored in the global storage array and the number of dynamic variables that the model wants stored in the global storage array

Call SetNumberofDiscreteControls $(0) \quad$ !The number of discrete

control functions set by this model (a value greater than zero requires the user to use

Solver 1: Powell's method)

Return

EndIf

!Do All of the First Timestep Manipulations Here - There Are No Iterations at the Intial Time

If (getIsStartTime()) Then

rho_glycol = getParameterValue $(1)$

k_glycol = getParameterValue(2)

$\mathrm{Nu}$ _glycol = getParameterValue (3)

cp_glycol = getParameterValue (4)

rho_water $=$ getParameterValue(5)

k_water = getParameterValue(6)

Beta_water $=$ getParameterValue(7) 


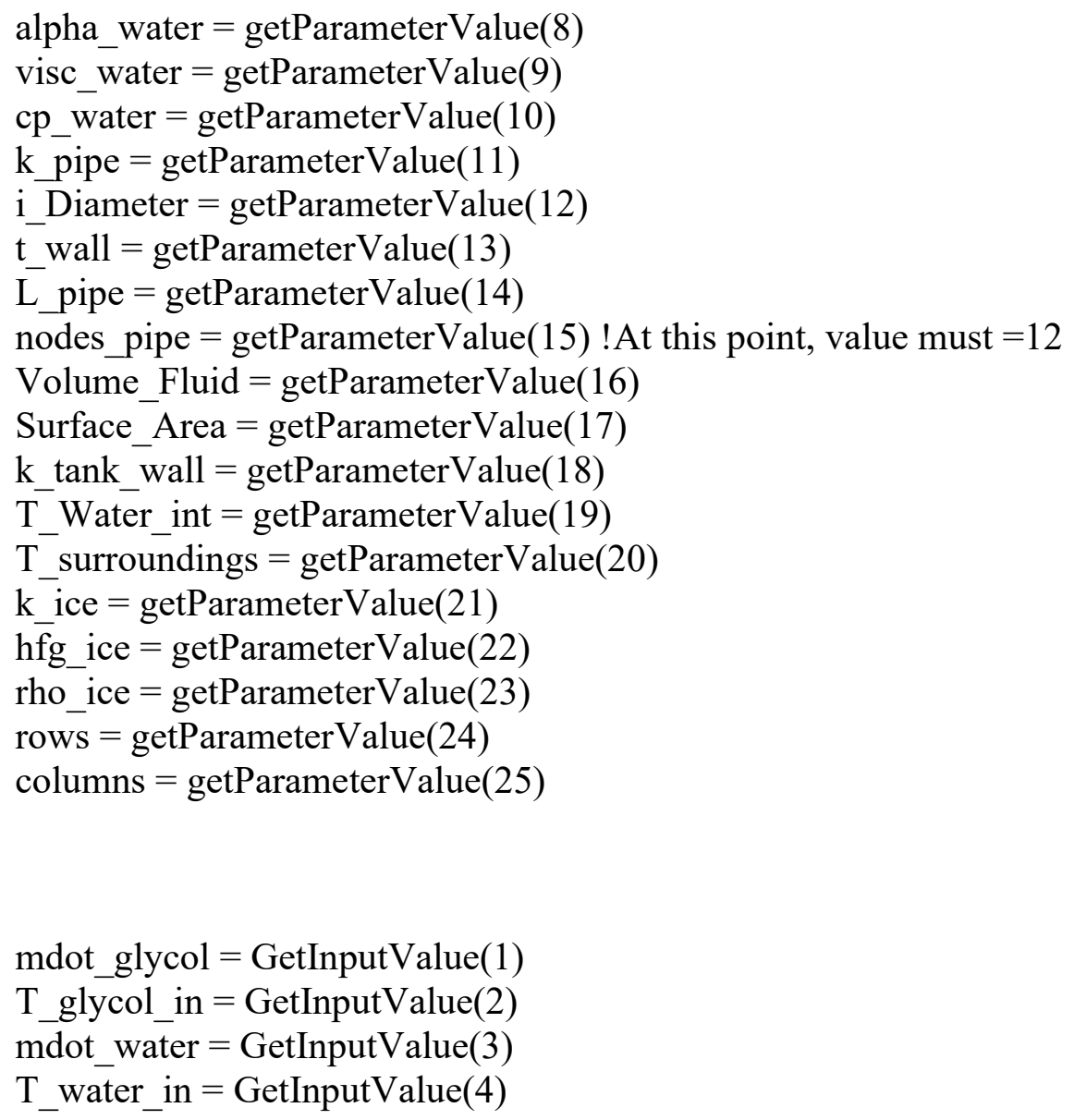

!Check the Parameters for Problems (\#,ErrorType,Text)

!Sample Code: If( PAR1 <= 0.) Call FoundBadParameter $(1$, 'Fatal','The first parameter provided to this model is not acceptable.')

!Set the Initial Values of the Outputs (\#,Value)

Call SetOutputValue(1, T_Water_int)! Tank Temperature

Call SetOutputValue(2, T_glycol_in)! glycol_T_out

Call SetOutputValue(3, mdot glycol)! mdot glycol

Call SetOutputValue(4, T_water_in)! water_T_out

Call SetOutputValue(5, mdot_water)! mdot_water

Call SetOutputValue $(6,0)$ ! mass ice

Call SetOutputValue $(7,0)$ ! Volume ice

Call SetOutputValue $(8,0)$ ! t ice_1

Call SetOutputValue $(9,0)$ ! t_ice_2

Call SetOutputValue $(10,0) !$ t _ice_3

Call SetOutputValue $(11,0)$ ! t ice_4

Call SetOutputValue $(12,0)$ ! t ice_5

Call SetOutputValue $(13,0)$ ! t _ ice_6 
Call SetOutputValue $(14,0) !$ t_ice_7

Call SetOutputValue $(15,0) !$ t _ice_8

Call SetOutputValue $(16,0)$ ! t ice 9

Call SetOutputValue $(17,0)$ ! t _ice_10

Call SetOutputValue $(18,0) ! t$ ice_11

Call SetOutputValue $(19,0) ! \mathrm{t}$ ice_12

Call SetOutputValue $(20,0)$ ! q stored

!If Needed, Set the Initial Values of the Dynamic Storage Variables (\#,Value) do $\mathrm{i}=1,96$

Call SetDynamicArrayValueThisIteration $(i, 0)$

enddo

do $\mathrm{h}=1,12$

energy_array $(\mathrm{h})=0$

delta_mass_array $(\mathrm{h})=0$

delta_volume_array $(\mathrm{h})=0$

mass_ice_array $(\mathrm{h})=0$

volume_ice_array $(\mathrm{h})=0$

t_ice_array $(\bar{h})=0$

mass_ice $=0$

end do

Call SetDynamicArrayValueThisIteration(97,T_Water_int)

Call SetDynamicArrayValueThisIteration(98,Volume_Fluid)

Call SetDynamicArrayValueThisIteration $(99,0)$

Call SetDynamicArrayValueThisIteration $(100,0)$

Return

EndIf

!ReRead the Parameters if Another Unit of This Type Has Been Called Last

If(getIsReReadParameters()) Then

!Read in the Values of the Parameters from the Input File

rho_glycol = getParameterValue (1)

k_glycol = getParameterValue (2)

$\mathrm{Nu}$ _glycol = getParameterValue(3)

cp_glycol = getParameterValue (4)

rho_water $=$ getParameterValue (5)

k_water $=$ getParameterValue (6)

Beta_water $=$ getParameterValue $(7)$

alpha_water $=$ getParameterValue $(8)$ 
visc_water $=$ getParameterValue(9)

cp_water $=$ getParameterValue $(10)$

$\mathrm{k}$ pipe $=$ getParameterValue $(11)$

i_Diameter $=$ getParameterValue (12)

t_wall = getParameterValue (13)

L_pipe $=$ getParameterValue (14)

nodes pipe $=$ getParameterValue(15) $!$ At this point, value must $=12$

Volume_Fluid = getParameterValue(16)

Surface_Area $=$ getParameterValue(17)

$\mathrm{k} \_$tank_wall $=$getParameterValue $(18)$

T_Water_int $=$ getParameterValue $(19)$

T_surroundings $=$ getParameterValue $(20)$

$\mathrm{k}$ ice $=$ getParameterValue $(21)$

$\mathrm{h} \overline{f g}$ ice = getParameterValue(22)

rho_ice $=$ getParameterValue $(23)$

rows $=$ getParameterValue $(24)$

columns $=$ getParameterValue $(25)$

EndIf

!Read the Inputs

mdot_glycol $=$ GetInputValue(1)

$\mathrm{T}$ glycol in $=$ GetInputValue $(2)$

mdot_water $=$ GetInputValue(3)

T_water_in $=$ GetInputValue(4)

T_Ref $=$ GetInputValue(5)

If(ErrorFound()) Return

$\mathrm{Li}=\mathrm{L} \_$pipe/nodes_pipe

r_o_pipe $=$ i_Diameter $/ 2+\mathrm{t}$ _wall

thick tank $=0.1$

$\mathrm{Pi}=3.14159$

T_Tank=GetDynamicArrayValuelasttimestep $(97)$

Volume_Fluid=GetDynamicArrayValuelasttimestep(98)

total_pipes $=$ rows $*$ columns

mdot_glycol_pipe=mdot_glycol/total_pipes

if ( $T$ _glycol_in $>T_{-}$Tank $)$then 
T_glycol_in=T_tank-2

end if

do $m=1,12$ !Change Node Here

$\mathrm{t} \_$ice_array $(\mathrm{m})=$ GetDynamicArrayValuelasttimestep $(\mathrm{m}+84)$

end do

Tin_i=T_glycol_in

if (mdot_glycol_pipe $>0$ ) then

do nodes $=1,12$ ! Change Node Here

Ts $=-2$

do itteration $=1,15$

T_Film $=($ Ts + T_Tank $) / 2$

k_water $=0.001 \overline{6}^{*} \mathrm{~T} \_$Film +0.5704

cp_water $=(0.0599 * T$ Tilm*T_Film-2.9726*T_Film +4216.4$) / 1000$

Beta_Water $=-(0.2053 * \mathrm{~T}$ Film*T_Film $+3.6803 * \mathrm{~T}$ _Film +39.622$) / 1000000$

alpha_water $=(0.045 * \mathrm{~T}$ Film $+13 . \overline{5} 37) / 1000000000$

visc_water $=\left(-0.0329 * \mathrm{~T}_{-}\right.$Film +1.6881$) / 1000000$

$\mathrm{R} 1=\overline{1} /\left(2 * \mathrm{Pi}^{*}\left(\mathrm{i} \_\right.\right.$Diameter $\left./ 2\right) * \mathrm{Li}^{*} \mathrm{Nu}$ glycol$* \mathrm{k}$ glycol/i_Diameter $)$

$\mathrm{R} 2=\log \left(\left(\left(\mathrm{i} \_\right.\right.\right.$Diameter/2)+t_wall $) /\left(\mathrm{i} \_\right.$Diameter/2) $) /\left(2 * \mathrm{Pi}^{-} \mathrm{k} \_\right.$pipe $\left.* \mathrm{Li}\right)$

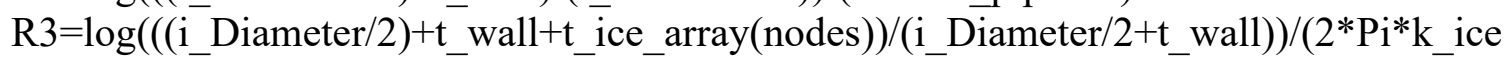
*Li)

$\mathrm{Ra}=9.81 *$ Beta_water*(Ts-

T_tank $) *($ i_Diameter $+2 *$ t_wall $+2 *(t$ _ice_array $($ nodes $))) * * 3 /($ visc_water*alpha_water $)$

$\mathrm{C}=.48$

$\mathrm{n}=.25$

if (Ra .LT. 0.01) then

$\mathrm{C}=0.675$

$\mathrm{n}=0.148$

else if (Ra .GE. 0.01 .AND. Ra .LT. 100) then

$\mathrm{C}=1.02$

$\mathrm{n}=.148$

else if (Ra .GE. 100 .AND. Ra .LT. 10000) then

$\mathrm{C}=0.85$

$\mathrm{n}=0.188$

else

$\mathrm{C}=.48$

$\mathrm{n}=.25$

end if

$\mathrm{R} 4=1 /\left(2 * \mathrm{Pi} *\left(\left(\mathrm{i} \_\right.\right.\right.$Diameter$\left./ 2\right)+\mathrm{t} \_$wall $+\mathrm{t} \_$ice_array $($nodes $\left.)\right) * \mathrm{Li}^{*} \mathrm{C} * \mathrm{Ra}^{*} * \mathrm{n} * \mathrm{k} \_$water/(i_Diam 
eter $+2 *$ t_wall $+2 *(t$ ice_array $($ nodes $))))$

$\mathrm{R}$ Total $=\mathrm{R} 1+\mathrm{R} 2+\mathrm{R} 3+\mathrm{R} 4$;

Tout_i=T_tank-(exp(-1/(mdot_glycol_pipe*cp_glycol*1000*R_Total) $\left.) *\left(T \_t a n k-T i n \_i\right)\right)$

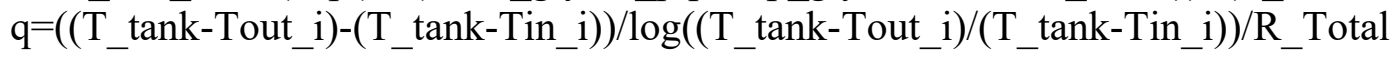

$\mathrm{T} 1 \_$in $=\mathrm{R} 1 * \mathrm{q}+\mathrm{Tin} \_\mathrm{i}$

T1_out $=\mathrm{R} 1 * \mathrm{q}+$ Tout_i

$\mathrm{T} 2$ in $=\mathrm{R} 2 * \mathrm{q}+\mathrm{T} 1$ in

$\mathrm{T} 2 \_$out $=\mathrm{R} 2 * \mathrm{q}+\mathrm{T} 1$ _out

$\mathrm{T} 3$ - in $=\mathrm{R} 3 * \mathrm{q}+\mathrm{T} 2$ in

T3_out $=\mathrm{R} 3{ }^{*} \mathrm{q}+\mathrm{T} 2$ _out

$\mathrm{Ts}=\left(\mathrm{T} 3 \_\right.$in $+\mathrm{T} 3 \_$out $) / 2$

end do

$\mathrm{q} \operatorname{array}($ nodes $)=0$

$\mathrm{q}$ array $($ nodes $)=\mathrm{q}$

T_Surface_Array(nodes) $=$ Ts

t_out_array(nodes) $=$ Tout_i

Tin_i=Tout_i

end do

q total $=0$

do $\mathrm{i}=1,12$ ! Change Node Here

$\mathrm{q} \_\mathrm{i}=\mathrm{q} \_\operatorname{array}(\mathrm{i})$

$q \_$total $=q \_$total $+q \_i$

enddo

else !Set energy transfered to tank to 0 if flown rate is 0

do $i=1,12$ ! Change Node Here

q_array $(i)=0$

q_total $=0$

enddo

end if

!Calculating the amount of energy leaving tank through water flow

if (mdot_water $>0$ ) then

q_out=mdot_water*cp_water*1000*(T_Tank-T_water_in)

energy_out=q_out ${ }^{*}$ Timestep $* 3600$

else

q_out $=0$

energy_out $=0$ 
end if

energy $=\mathrm{q} \_$total $*$ total_pipes $*$ Timestep $* 3600$;

q_loss $=$ surface_area*(T_surroundings-T_tank $) * k$ tank_wall/(thick_tank $)$;

e_loss $=\mathrm{q} \_$loss $*$ Timestep $* 3600$;

net_energy $=$ energy+energy_out-e_loss !This is the net energy across all pipes

net_energy_pipe $=$ net_energy/total_pipes !Calculate the net energy per pipe

if (net_energy $>0$ ) then !net result is system charging

Energy_sensible=net_energy

!Calculate the sensible change

delta_T_tank=Energy_sensible/(rho_water*Volume_fluid*cp_water*1000);

T_tank_test $=$ T_tank-delta_T_tank;

if ( $T$ _tank_test $>0$ ) then !Check to see if tank goes below 0 - If not then tank changes based on sensible change

T_Tank=T_Tank_test

Call SetDynamic ArrayValueThisIteration(97,T_Tank) !saves tank temperature to the global variable for next itteration

else

e_zero $=$ T_Tank*cp_water*1000*rho_water*Volume_fluid; !Determine the amount of energy required to get to 0 ( $\mathrm{T}_{-}$Tank is the value from previous time step)

Ice_after_sensible $=($ energy_sensible-e_zero $) /$ total_pipes !Determine how much energy from each pipe needs to be converted to ice

$\mathrm{T}_{-}$Tank $=0$ ! Set tank at 0 as water can't go below that

end if

do $\mathrm{k}=1,12$ ! Change Node Here

!retrieve total mass and volume of ice

mass_ice_array $(\mathrm{k})=$ getdynamicarrayvaluelasttimestep $(\mathrm{k}+60)$

volume_ice_array $(\mathrm{k})=$ getdynamicarrayvaluelasttimestep $(\mathrm{k}+72)$

delta mass array $(\mathrm{k})=0$ ! Set increase to 0

delta_volume_array $(\mathrm{k})=0$

e_i $=\left(\mathrm{q} \_\right.$array $(\bar{k}) / q$ total $) *(($ Energy_Ice_int $)+$ Ice_after_sensible $)$ !calcualte the amount of energy for each node getting converted to ice

energy_array $(\mathrm{k})=\mathrm{e} \_\mathrm{i}$

delta_mass_array $(\mathrm{k})=$ energy_array $(\mathrm{k}) / \mathrm{hfg}$ ice !Change in mass for each node along the length of each pipe

delta_volume_array $(\mathrm{k})=$ delta_mass_array $(\mathrm{k}) / \mathrm{rho}$ _ice !Change in Volume for each node along the length of each pipe

mass_ice_array $(\mathrm{k})=$ mass_ice_array $(\mathrm{k})+$ delta_mass_array $(\mathrm{k})$ ! Total mass of ice on each

node for each node along the length of each pipe 
!volume_ice_array $(\mathrm{k})=$ volume_ice_array $(\mathrm{k})+$ delta_volume_array $(\mathrm{k})$ ! Total Volume for each node along the length of each pipe

!t ice $\operatorname{array}(\mathrm{k})=\operatorname{sqrt}\left(\right.$ volume ice $\operatorname{array}(\mathrm{k}) /\left(\mathrm{Pi} \mathrm{i}^{*} \mathrm{Li}\right)+\mathrm{r}$ o pipe $\left.{ }^{* * 2}\right)-\mathrm{r}$ o pipe !Thickness of ice for each node along the length of each pipe

delta_mass $=0$

call setDynamicArrayValueThisIteration( $(\mathrm{k}+60$,mass_ice_array $(\mathrm{k}))$ !Determines the total mass of ice formed at each node

Call SetDynamicArrayValueThisIteration(97,T_Tank) !set tank temperature to 0 end do

else !net energy is negative and system is discharging - calcuate ice melt or tank temp increase

mass_melted $=(-1) *$ net_energy $/$ hfg_ice

mass_melted_node=mass_melted/(total_pipes*12)!Change Node Here

t_energy_A_ice $=0$

do $\mathrm{f}=1,1 \overline{2}$ ! Change Node Here

energy_after_Ice(f) $=0$

mass_ice_array $(\mathrm{f})=$ getdynamicarrayvaluelasttimestep $(\mathrm{f}+60)$

volume_ice_array $(\mathrm{f})=$ getdynamicarrayvaluelasttimestep $(\mathrm{f}+72)$

delta_mass_array $(\mathrm{f})=0$

delta_mass_array $(\mathrm{f})=$ mass_melted_node

if (mass_ice_array $(\mathrm{f})>$ mass_melted_node) then

mass_ice_array $(\mathrm{f})=$ mass_ice_array(f)-mass_melted_node

else

mass_ice_array $(\mathrm{f})=0$

energy_after_Ice $(\mathrm{f})=\left(\text { mass_ice_array }(\mathrm{f})-\mathrm{mass} \_ \text {melted_node }\right)^{*} \mathrm{hfg}$ ice* $(-1) *$ total_pipes end if

call setDynamicArrayValueThisIteration(f+60,mass ice array(f)) !Determines the total mass of ice formed at each node

end do

do $\mathrm{k}=1,12$ ! Change Node Here

t_energy_A_ice $=t$ _energy_A_ice+energy_after_ice(k)

end do

T_Tank $=$ T_Tank+t_energy_A_ice/(Volume_Fluid*rho_water*cp_water*1000)

delta mass $=0$

Call $\bar{S}$ etDynamicArrayValueThisIteration(97,T_Tank) !set tank temperature to 0

end if

!Calculating Total stored energy

Volume ice $=0$

mass_ice $=0$ 
do $\mathrm{j}=1,12$ ! Change Node Here

Volume_ice=Volume_Ice_array(j)+volume_ice

mass_ice $=$ mass_ice + mass_ice_array $(j)$

delta_mass $=$ delta_mass_array $(\bar{j})+$ delta_mass

volume_ice_array $(\mathrm{j})=$ mass_ice_array $(\mathrm{j}) /$ rho_ice !Total Volume for each node along the length of each pipe

$\mathrm{t}$ ice_array $(\mathrm{j})=\mathrm{sqrt}\left(\right.$ volume_ice_array $\left.(\mathrm{j}) /(\mathrm{Pi} * \mathrm{Li})+\mathrm{r} \_\mathrm{o} \_p i p e^{* *} 2\right)-\mathrm{r} \_\mathrm{o} \_$pipe !Thickness of ice for each node along the length of each pipe

call setDynamicArrayValueThisIteration(j+72,volume_ice_array(j)) !Determines the total volume of ice formed at each node

call setDynamicArrayValueThisIteration $(j+84, t$ _ice_array $(j))$ !Determines the thickeness

of ice formed at each node

end do

total_mass_ice=mass_ice*total_pipes

Total_Volume_Ice $=\overline{\text { Volume }}$ ice*total_pipes

total_delta_mass ice=delta_mass*total pipes

call setDynamicArrayValueThisIteration(99, Total_Volume_Ice)

call setDynamicArrayValueThisIteration(100, total_mass_ice)

Volume_displaced $=$ total_delta_mass_ice/rho_water;

!Volume_Fluid=Volume_Fluid-Volume_displaced

call setDynamicArrayValueThisIteration(98,Volume_Fluid)

sensible $=\left(T \_\right.$Ref-T_Tank $) *$ cp_water*1000*Volume_Fluid*rho_water

latent=total_mass_ice*hfg_ice

q stored total $=($ sensible + latent $) / 1000 * 0.000277778$ !Calculated in $\mathrm{kwh}$

!Set the Outputs from this Model (\#,Value)

Call SetOutputValue(1, T_Tank)! Tank Temperature

Call SetOutputValue(2, Tout_i)! glycol_T_out

Call SetOutputValue(3, mdot_glycol)! mdot_glycol

Call SetOutputValue(4, T Tank)! water T out

Call SetOutputValue(5, mdot_water)! mdot_water

Call SetOutputValue(6, total_mass_ice)! mass ice

Call SetOutputValue(7, total_Volume_ice)! Volume ice

Call SetOutputValue $(8, \mathrm{t}$ ice array(1)) ! t ice 1

Call SetOutputValue $(9, \mathrm{t}$ ice array(2))! $\mathrm{t}$ ice 2

Call SetOutputValue(10, $t$ tice_array(3))! t

Call SetOutputValue(11, t_ice_array(4))! t_ice_4

Call SetOutputValue(12, t_ice_array(5))! t ice_5

Call SetOutputValue(13, t ice_array(6))! t ice_6

Call SetOutputValue(14, t_ice_array(7))! t_ice_7 
Call SetOutputValue(15, t_ice_array(8))! t ice_8

Call SetOutputValue $(16, t$ tice_array $(\overline{9})) !$ t_ice_9

Call SetOutputValue $(17, \mathrm{t}$ _ice_array(10)) ! $\mathrm{t}$ _ice_10

Call SetOutputValue $(18, \mathrm{t}$ _ice_array(11)) ! t_ice_11

Call SetOutputValue $(19, \mathrm{t}$ _ice_array(12))! t_ice_12

Call SetOutputValue(20, net_energy)! q_stored_timestep

Call SetOutputValue(21, q_stored_total)! total energy stored in the system

Call SetOutputValue(22, mass_melted)! R_Total

Call SetOutputValue(23, t_energy_A_ice)! sensible

Call SetOutputValue $(24$, R4) ! latent

Call SetOutputValue(25, R_Total)! q_loss

!If Needed, Store the Desired Disceret Control Signal Values for this Iteration (\#,State)

!Sample Code: Call SetDesiredDiscreteControlState $(1,1)$

!If Needed, Store the Final value of the Dynamic Variables in the Global Storage Array (\#,Value)

!Sample Code: Call SetDynamicArrayValueThisIteration(1,T_FINAL_1)

Return

End

!-----------------




\section{Appendix F Schematics of TRNSYS Models used to model the full building}

This appendix shows images of the overall TRNSYS models used within this study, as well as the sub-system modelled.

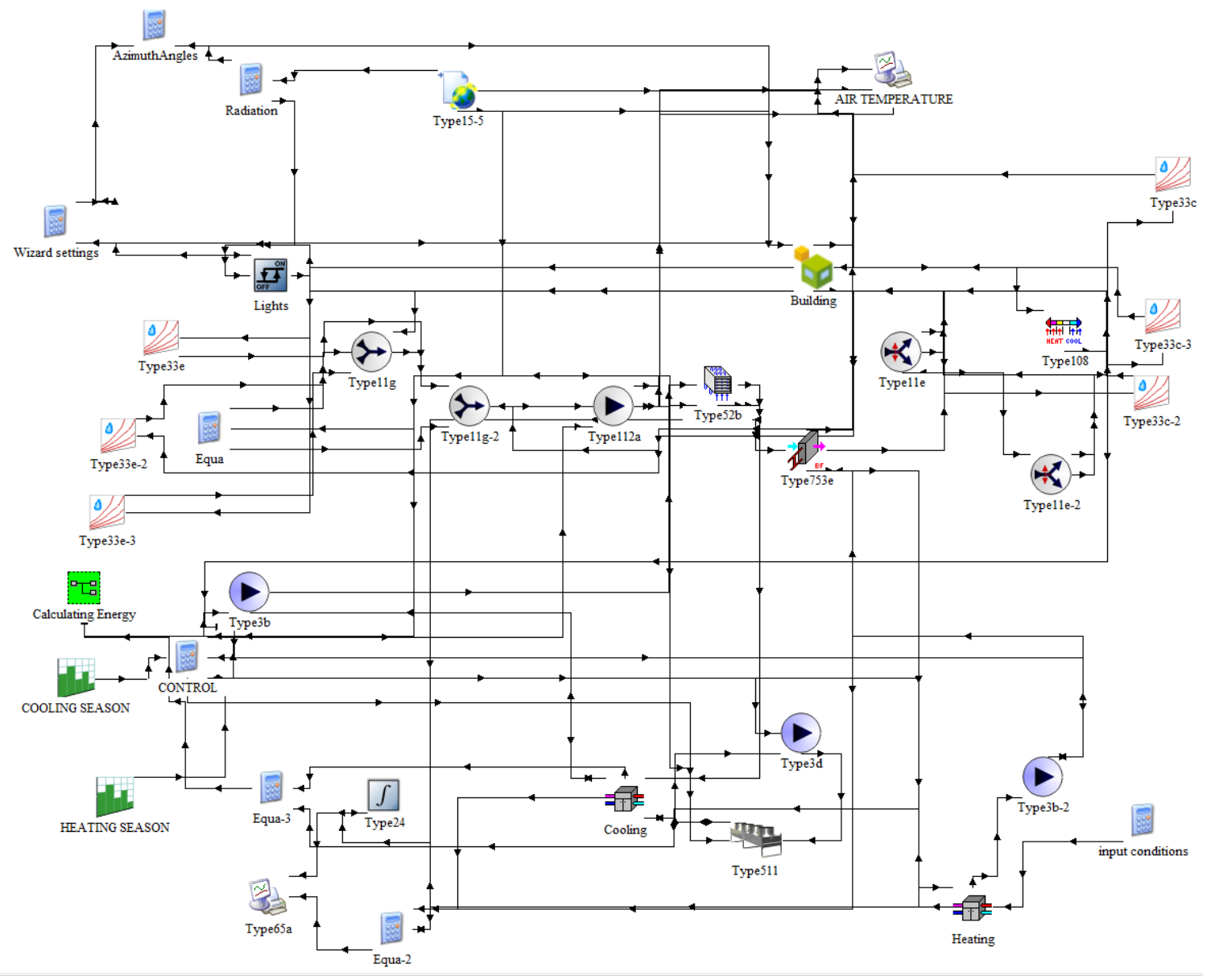

Figure F-1: TRNSYS model for calculating the baseline energy consumption using the liquid to liquid heat pump 


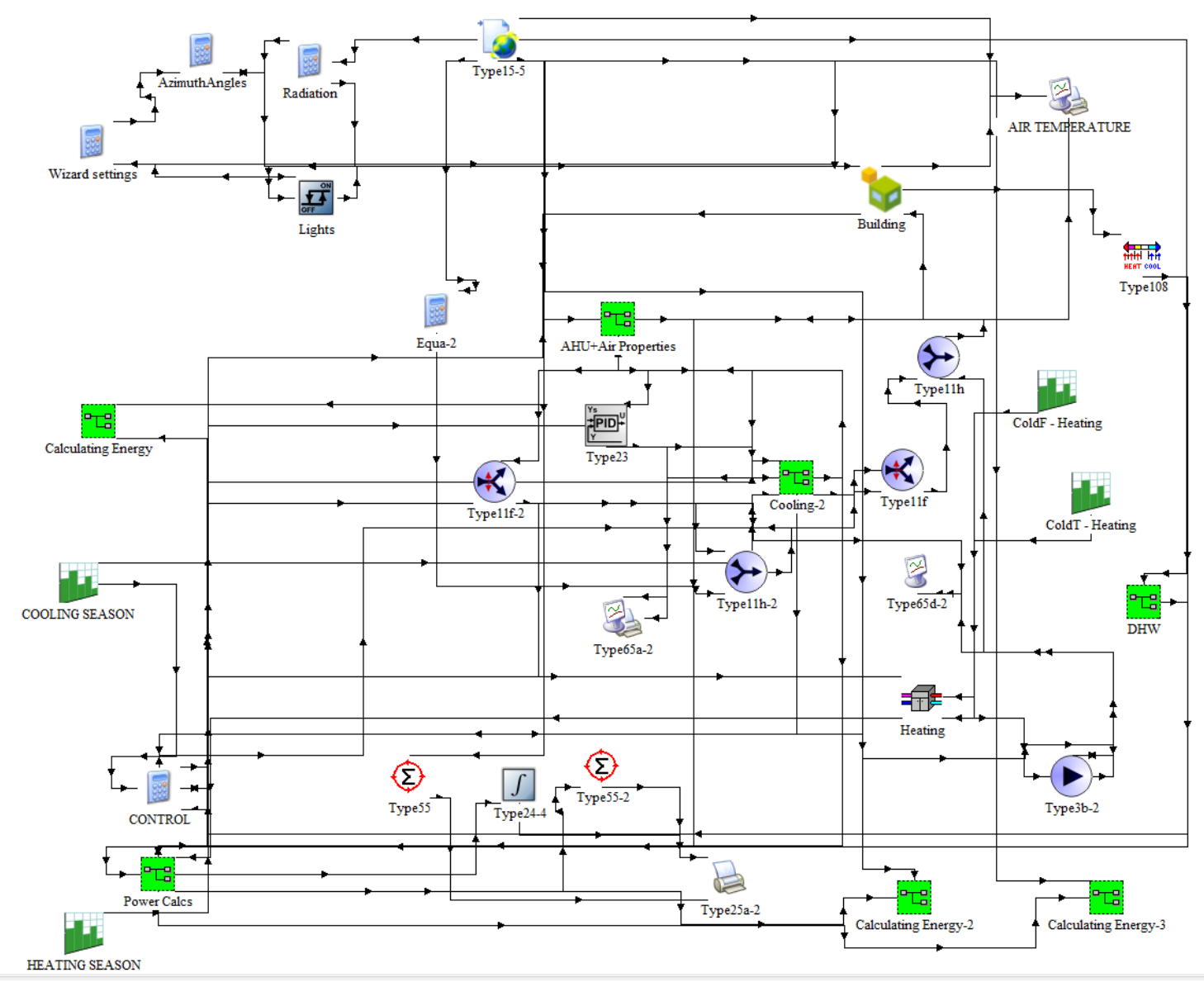

Figure F-2: TRNSYS model for the complete system using thermal storage and domestic hot water 


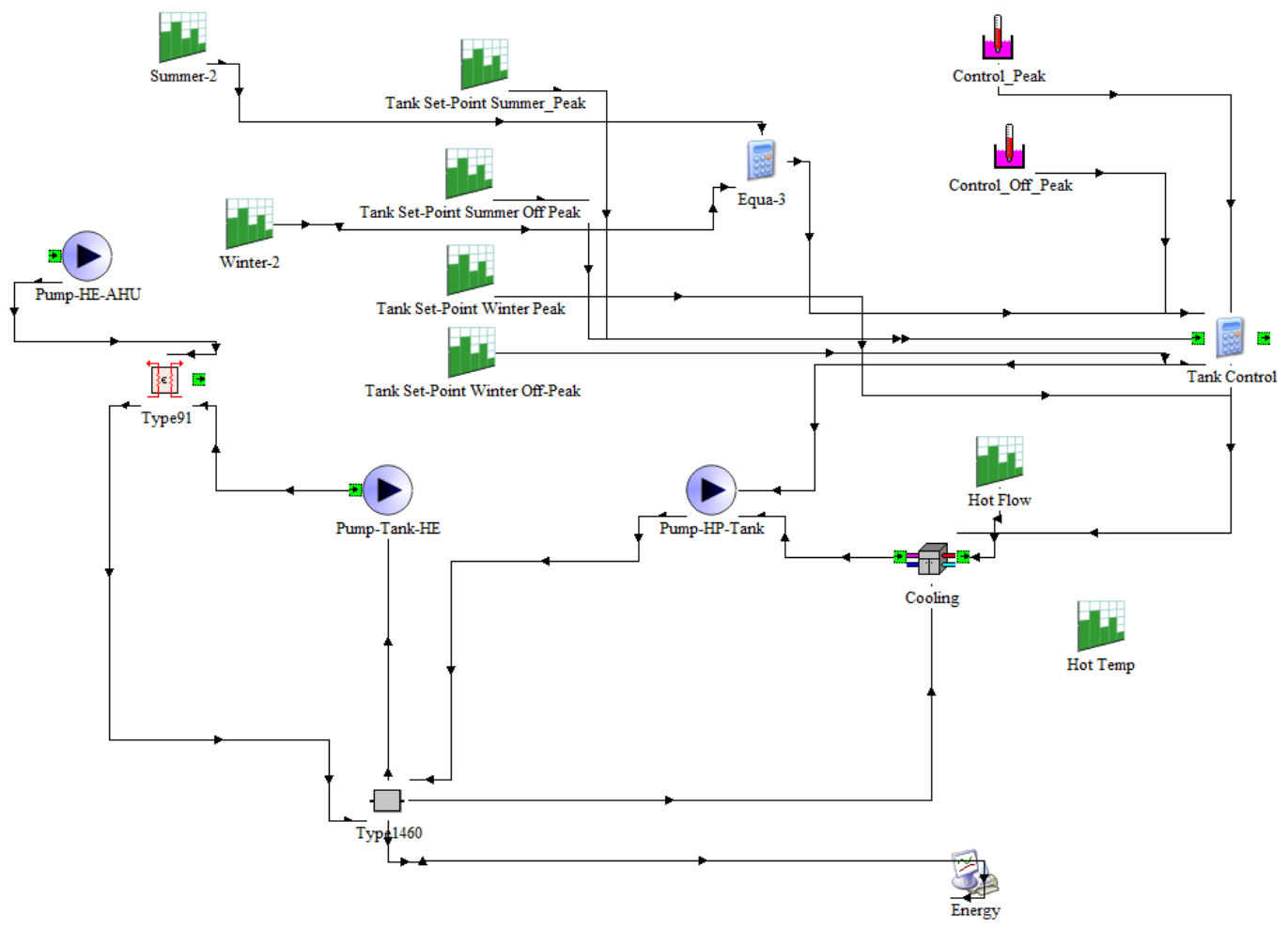

Figure F-3: TRNSYS model of the cold thermal storage, including the control components and the ice storage (Type 1460)

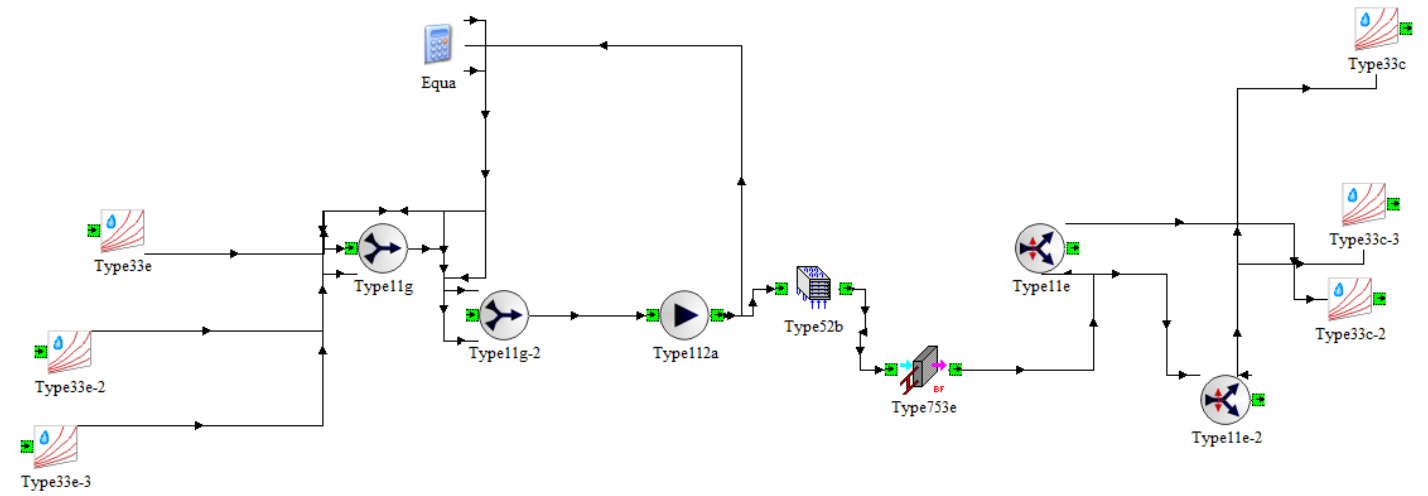

Figure F-4: TRNSYS model of the air handling unit, including the mixing of the return air from each zone and the splitting of the air to each of the three zones 


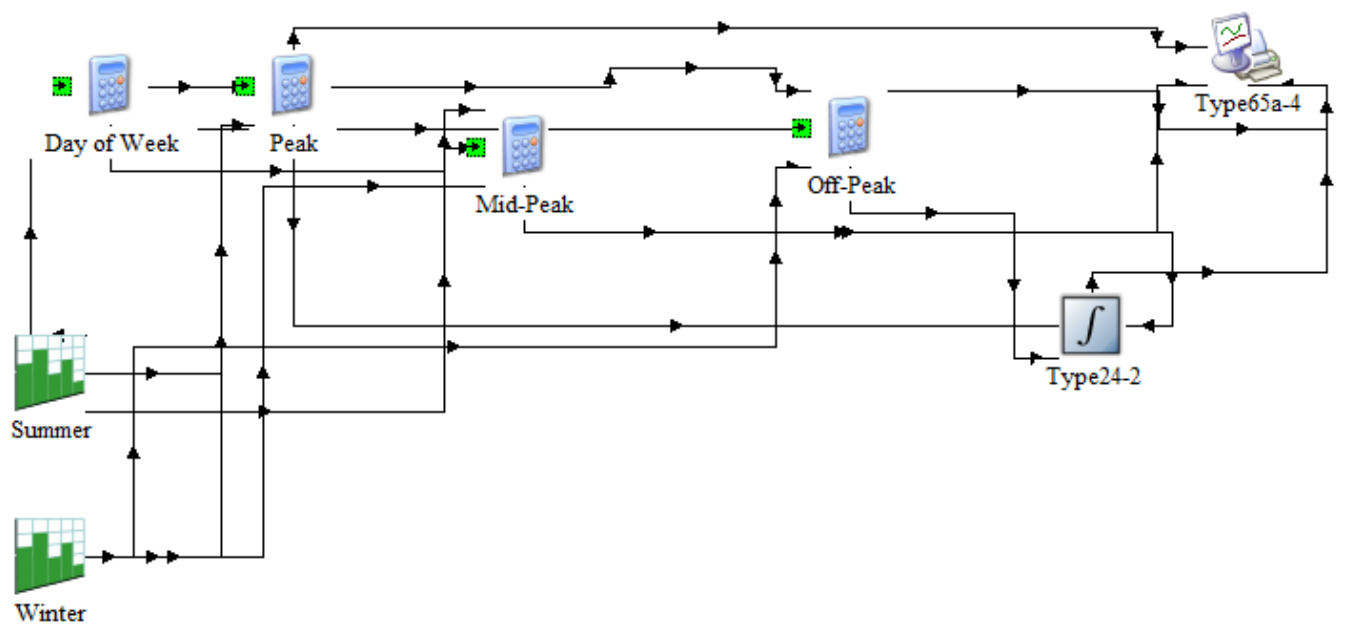

Figure F-5: TRNSYS model used to calculate the time energy is used and the total consumption, and whether it is peak, mid peak or off peak periods

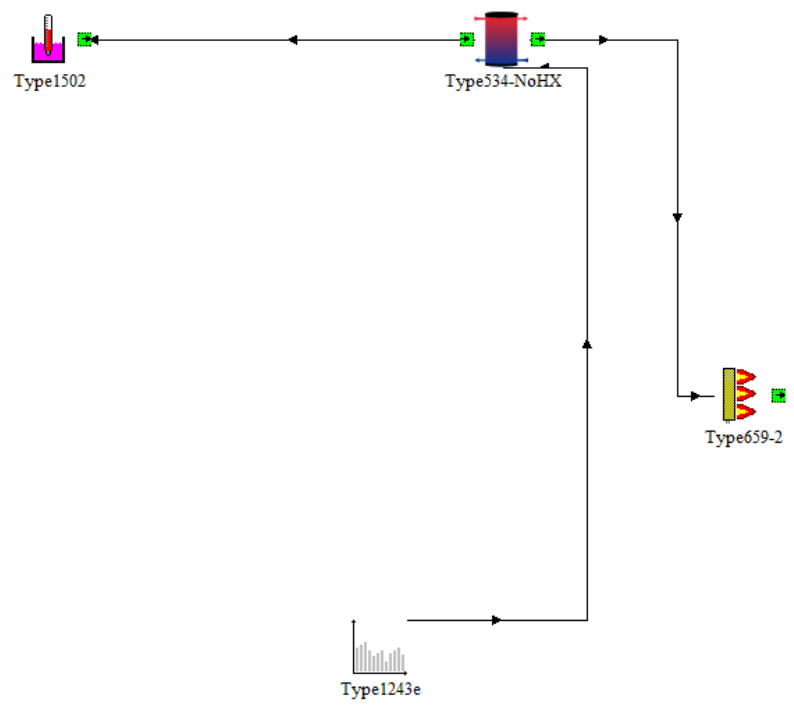

Figure F-6: TRNSYS model of the domestic hot water system, using a preheat tank and instantaneous heater 